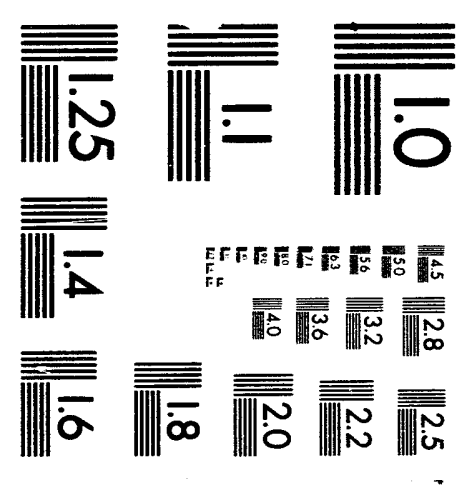



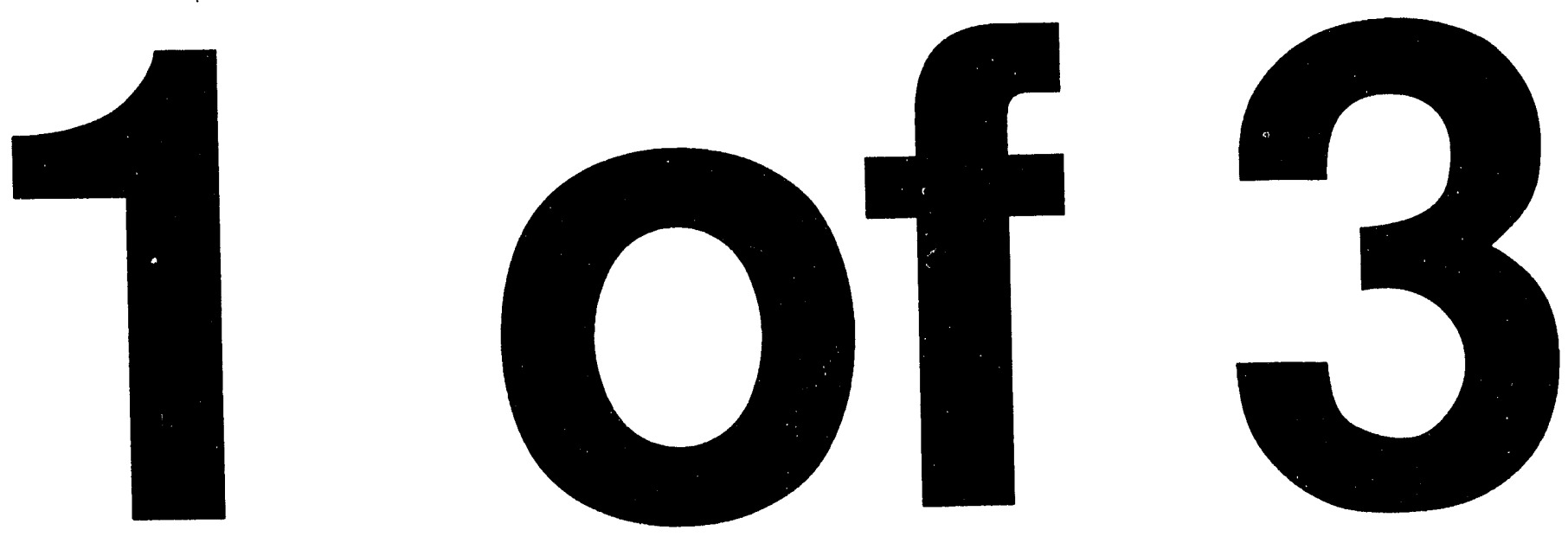
SAND93-1377 •UC-610

Unlimited Release

Printed October 1993

\title{
MELCOR 1.8.2 Assessment: The DF-4 BWR Damaged Fuel Experiment
}

\author{
Timothy J. Tautges
}

Prepared by

Sandla National Laboratorles

Albuquerque, New Mexico 87185 and Livermore, California 94550

for the United States Department of Energy

under Contract DE-AC04-94AL85000 
Issued by Sandia National Laboratories, operated for the United States Department of Energy by Sandia Corporation.

NOTICE: This report was prepared as an account of work sponsored by an agency of the United States Government. Neither the United States Government nor any agency thereof, nor any of their employees, nor any of their contractors, subcontractors, or their employees, makes any warranty, express or implied, or assumes any legal liability or responsibility for the accuracy, completeness, or usefulness of any information, apparatus, product, or process disclosed, or represents that its use would not infringe privately owned rights. Reference herein to any specific commercial product, process, or service by trade name, trademark, manufacturer, or otherwise, does not necessarily constitute or imply its endorsement, recommendation, or favoring by the United States Government, any agency thereof or any of their contractors or subcontractors. The views and opinions expressed herein do not necessarily state or reflect those of the United States Government, any agency thereof or any of their contractors.

Printed in the United States of America. This report has been reproduced directly from the best available copy.

Available to DOE and DOE contractors from Office of Scientific and Technical Information

PO Box 62

Oak Ridge, TN 37831

Prices available from (615) 576-8401, FTS 626-8401

Available to the public from

National Technical Information Service

US Department of Commerce

5285 Port Royal Rd

Springfield, VA 22161

NTIS price codes

Printed copy: A10

Microfiche copy: A01 
SANI)93-1377

Unlimited Release

Printed October 1993

\title{
MELCOR 1.8.2 ASSESSMENT: THE DF-4 BWR DAMAGED FUEL EXPERIMENT
}

\author{
Timothy J. Tautges \\ Thermal/Hydraulic: Analysis Department \\ Sandia National Laboratories \\ Albuquerque, NM 87185
}

\begin{abstract}
MELCOR is a fully integrated, engineering-level computer code being developed at Sandia National Laboratories for the USNRC, that models the entire spectrum of severe accident phenomena in a unified framework for both BWRs and PWRs. As a part of an ongoing assessment program, MELCOR has been used to model the ACRR in-pile DF-4 Damaged Fuel experiment. I)F-4 provided data for early phase melt progression in BWR fuel assiemblies. particularly for phenomena associated with eutectic interactions in the BWR control blade and zircaloy oxidation in the canister and cladding.

MELCOR provided good agreement with experimental data in the key areas of eutectic material behavior and canister and claclding oxidation. Several shortcomings associated with the MELCOR modeling of BWR geometries were found and corrected. Twenty-five sensitivity studies were performed on COR, HS and CVH paral.ueters. These studies showed that the new MEI,COR euteclics model played an important role in predicting control biade behavior. 'These studies revealed slight time step dependence and no machine dependencies. Comparisons made with the results from four best-estirnate codes showed that MELCOR did as well as these codes in matching DF-4 experimental data.
\end{abstract}

\section{MASTER}




\section{Contents}

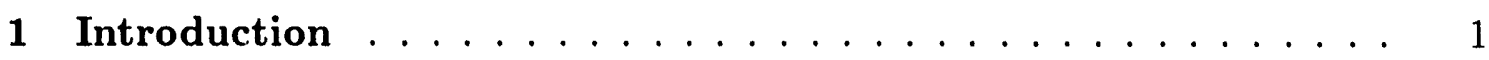

2 Facility and Test Description . . . . . . . . . . . . . 3

2.1 Facility Description . . . . . . . . . . . . . . . . . . 3

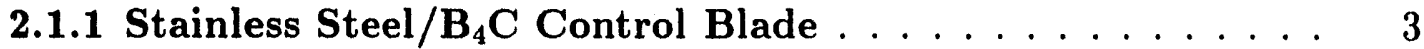

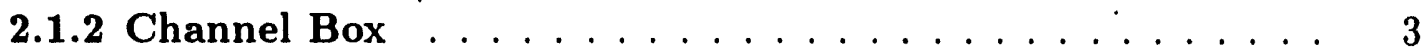

2.1.3 Fuel Rods . . . . . . . . . . . . . . . . . . . . . . 6

2.1.4 Test Section Insulation and Radial Boundary . . . . . . . . 7

2.1.5 Other DF-4 Experiment Components . . . . . . . . . . 7

2.2 Test Description . . . . . . . . . . . . . . . . . . . . . 11

3 MELCOR Input Model $\ldots \ldots \ldots \ldots \ldots \ldots$

3.1 Geometry . . . . . . . . . . . . . . . . . . . 13

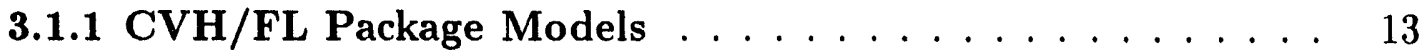

3.1.2 COR Package Model . . . . . . . . . . . . . . . . . . . 14

3.1.3 Heat Structures Model . . . . . . . . . . . . . . . . . . . 17

3.2 Test Bundle Initial and Boundary

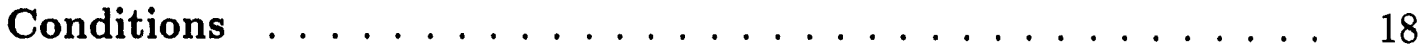

4 MELCOR Base Case Calculation Results . . . . . . . . . . . . 24

4.1 Temperature Response . . . . . . . . . . . . . . . . . . . . . . . . . . 24

4.1.1 Control Blade . . . . . . . . . . . . . . . . . . 24

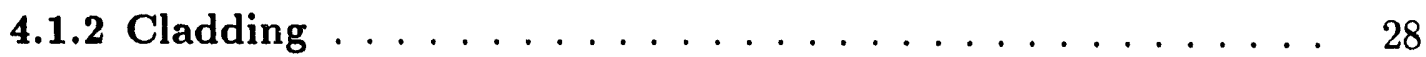

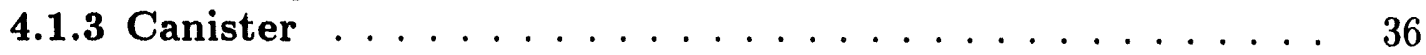

4.1.4 Component Temperature Response Overview . . . . . . . . 38

4.2 Melting and Relocation . . . . . . . . . . . . . . . 40

4.2.1 Initial State . . . . . . . . . . . . . . . . . . . 40

4.2.2 Early Oxidation Subphase (7450-7625 seconds) $\ldots \ldots . .40$

4.2.3 Later Oxidation Subphase (7650-7700 seconds) . . . . . . . 51

4.2.4 Final State . . . . . . . . . . . . . . . . . 52 
4.3 Hydrogen Production $\ldots \ldots \ldots \ldots$

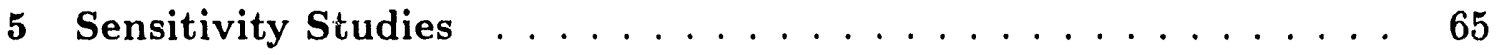

5.1 General Core Package Studies . . . . . . . . . . . . . 65

5.1.1 Convective Heat Transfer Coefficients . . . . . . . . . . 65

5.1.2 Core Package Material Properties . . . . . . . . . . . . 66

5.1.3 Core Component Initial Temperatures . . . . . . . . . 66

5.1.4 Core Component View Factors . . . . . . . . . . . . . 72

5.1.5 Canister Mass and Area Partition . . . . . . . . . . . 73

5.1.6 Minimum Component Mass . . . . . . . . . . . . . . . 77

5.1.7 Candling Heat Transfer Coefficient . . . . . . . . . . 77

5.1.8 Minimum Oxide Shell Thickness . . . . . . . . . . . . . . 82

5.1.9 Heat Structure Boundary Fluid Temperature

Option . . . . . . . . . . . . . . . . . 90

5.2 Debris Behavior Studies . . . . . . . . . . . . . . 95

5.2.1 Debris Porosity . . . . . . . . . . . . . . . . . . . 95

5.2 .2 Debris Diameter .................... . . 100

5.3 Oxidation Studies . . . . . . . . . . . . . . . . . . 100

5.3.1 $\mathbf{B}_{4} \mathbf{C}$ Oxidation Model . . . . . . . . . . . . . . . . 100

5.3.2 Oxidation Reaction Rate Transition Temperature . . . . . . 109

5.4 Eutectic Model Studies . . . . . . . . . . . . . . . . . 110

5.4.1 Deactivate Eutectic Model . . . . . . . . . . . . . . . 110

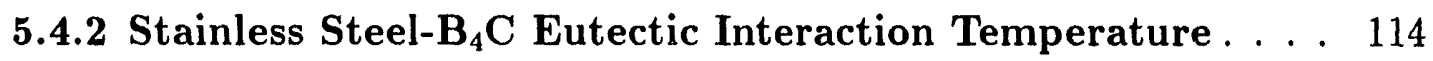

5.5 Thermal/Hydraulic Initial and Boundary

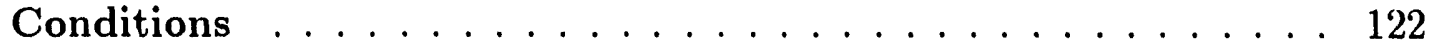

5.5.1 Steam Mass Flow Rate . . . . . . . . . . . . . . . . . 122

5.5.2 Steam Inlet Temperature . . . . . . . . . . . . . . 129

5.5.3 Porous Zirconia Thermal Conductivity . . . . . . . . . . . . . 129

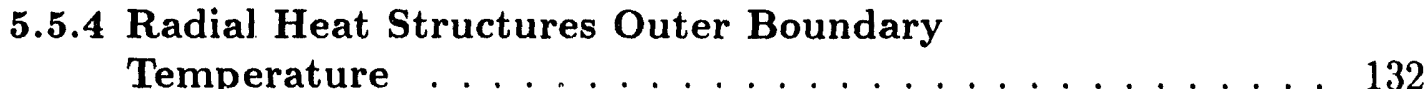

5.5.5 Bundle Fission Power . . . . . . . . . . . . . . . . 132

5.6 General Heat Structures Package Studies . . . . . . . . . . . . 139

5.6.1 HS Radiation Model Option . . . . . . . . . . . . . . 139

5.6.2 Heat Structures Emissivities . . . . . . . . . . . . 139

5.7 COR and CVH Nodalization . . . . . . . . . . . . . . . . 139

5.8 Time Step and Machine Dependency Studies . . . . . . . . . . . . 140

5.8.1 Time Step Study . . . . . . . . . . . . . . . . . . . . 140

5.8.2 Machine Dependency Study . . . . . . . . . . . . . . . 144

6 Comparison With Other Codes . . . . . . . . . . . 166

6.1 Temperature Response . . . . . . . . . . . . . . . . . . 167

6.2 Hydrogen Production . . . . . . . . . . . . . . 173

6.3 Final State . . . . . . . . . . . . . . . . . 175 
7 Code Problems Identified $\ldots \ldots \ldots \ldots \ldots$

7.1 Heat Transfer From Heat Structures to

Channel Volume . . . . . . . . . . . . . . . . . . . 176

7.2 Divide By Zero in CVHBVT . . . . . . . . . . . . . . . . 176

7.3 Internal File Length in MPDFVL . . . . . . . . . . . . . . 177

7.4 Boundary Fluid Temperature Option in $\mathbf{d T} / \mathrm{dz} \ldots \ldots \ldots \ldots$

7.5 Radiation Heat Transfer in $\mathbf{d} \mathbf{T} / \mathrm{dz} \ldots \ldots \ldots \ldots \ldots \ldots$

7.6 COR Energy Error Due to Fission Power . . . . . . . . . . . 178

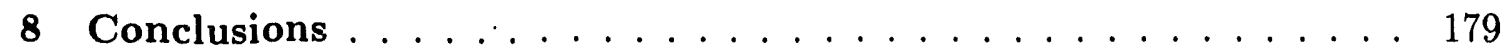

Refereı. ces/Bibliography . . . . . . . . . . . . . . . . . . 182

A Base Case Input Deck $\ldots \ldots \ldots \ldots \ldots \ldots \ldots$ 


\section{List of Figures}

2.1.1 Radial cross section of the DF-4 test bundle (from $[6]$ ). . . . . . . 4

2.1.2 Axial cross section of the DF-4 test bundle (from [6]) . . . . . . . . 5

2.1.3 Radial locations of bundle thermocouples (from $[6]$ ). . . . . . . . . 9

2.1.4 Mnemonic indicators for bundle thermocouple locations (from [6]). . . 10

2.2.1 Measured reactor power in the ACRR during the fuel damage phase of the DF-4 experiment. . . . . . . . . . . . . . . 11

2.2.2 Total inlet steam flow rate during the fuel damage phase of the DF-4

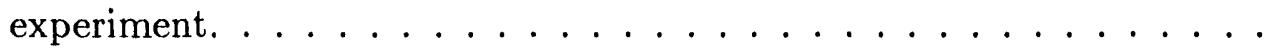

3.1.1 Control volume and flow path arrangement in the MELCOR DF-4 base case input model. . . . . . . . . . . . . . . . . .

3.1.2 Core and heat structures nodalization in the MELCOR DF-4 base case input model (upper boundary heat structure not shown). . . . . . . .

3.2.1 Empirical thermal conductivity correlation for porous $\mathrm{ZrO}_{2}$ (from [8]) compared to $23 \%$ of MELCOR thermal conductivity for this material.

3.2.2 Cell fission power coupling factors used in MELCOR, compared to data and the correlation derived from those data. Error bars on data values show the $95 \%$ confidence limits. . . . . . . . . . . . . . . 22

3.2.3 Steam inlet temperature used in MELCOR. . . . . . . . . . . . . . . 23

3.2.4 Constant heat structure outside surface temperature used in MELCOR compared to measured temperatures at several axial levels. TSJK05 is at the top of the bundle and TSJK20 is at the bottom. . . . . . . . .

4.1.1 Measured vs. calculated control blade temperatures at the $49.5 \mathrm{~cm}$ level. . . . . . . . . . . . . . . . . . . . 26

4.1.2 Measured vs. calculated control blade temperatures at the $36.8 \mathrm{~cm}$ level. . . . . . . . . . . . . . . . . . . . .

4.1.3 Measured vs. calculated control blade temperatures at the $25.4 \mathrm{~cm}$ level. . . . . . . . . . . . . . . . . . . . .

4.1.4 Measured vs. calculated control blade temperatures at the $9.6 \mathrm{~cm}$ level. . . . . . . . . . . . . . . . . . . . . . .

4.1.5 Measured vs. calculated fuel rod cladding temperatures at the $49.5 \mathrm{~cm}$ level. . . . . . . . . . . . . . . . . . . . . .

4.1.6 Measured vs. calculated fuel rod cladding temperatures at the $36.8 \mathrm{~cm}$ level. . . . . . . . . . . . . . . . . . . . 
4.1.7 Measured vs. calculated fuel rod cladding temperatures at the $25.4 \mathrm{~cm}$ level. . . . . . . . . . . . . . . . . . . . .

4.1.8 Measured vs. calculated fuel rod cladding temperatures at the $9.6 \mathrm{~cm}$ level. . . . . . . . . . . . . . . . . . . . .

4.1.9 Measured vs. calculated canister temperatures at the 49.5 and $36.8 \mathrm{~cm}$ levels. . . . . . . . . . . . . . . . . . .

4.1.10 Measured vs. calculated canister temperatures at the 25.4 and $9.6 \mathrm{~cm}$ levels. . . . . . . . . . . . . . . . . . .

4.1.11 Cladding temperatures at the four measured axial planes, for the base case input model. . . . . . . . . . . . . . . . .

4.1.12 Control blade temperatures at the four measured axial planes, for the base case input model. . . . . . . . . . . . . . . . . .

4.1.13 Component temperatures for the fuel, cladding, canister-b and other structure (control blade) components, at the $36.8 \mathrm{~cm}$ plane, for the base case input model. . . . . . . . . . . . . . . . . . .

4.1.14 Component temperatures for the fuel, cladding, canister-b. and other structure (control blade) components, at the $25.4 \mathrm{~cm}$ plane, for the base case input model. . . . . . . . . . . . . . . . . .

4.2.1 Initial locations (at 6000 seconds) of fuel and clad (upper left), particulate debris (upper right), canister-b (lower left) and other structure (lower right) component materials in DF-4. . . . . . . . . . . .

4.2.2 Locations of fuel and clad (upper left), particulate debris (upper right), canister-b (lower left) and other structure (lower right) component materials in DF-4 at 7450 seconds. . . . . . . . . . . . . . . . . .

4.2.3 Locations of fuel and clad (upper left), particulate debris (upper right), canister-b (lower left) and other structure (lower right) component materials in DF-4 at 7500 seconds. . . . . . . . . . . . . . . . . .

4.2.4 Locations of fuel and clad (upper left), particulate debris (upper right), canister-b (lower left) and other structure (lower right) component materials in DF-4 at 7550 seconds. . . . . . . . . . . . . . . . .

4.2.5 Locations of fuel and clad (upper left), particulate debris (upper right), canister-b (lower left) and other structure (lower right) component materials in DF-4 at 7600 seconds. . . . . . . . . . . . . . . . .

4.2.6 Locations of fuel and clad (upper left), particulate debris (upper right), canister-b (lower left) and other structure (lower right) component materials in DF-4 at 7625 seconds. . . . . . . . . . . . . . . . . . .

4.2.7 Locations of fuel and clad (upper left), particulate debris (upper right), canister-b (lower left) and other structure (lower right) component materials in DF-4 at 7650 seconds. . . . . . . . . . . . . . . . .

4.2.8 Locations of fuel and clad (upper left), particulate debris (upper right), canister-b (lower left) and other structure (lower right) component materials in DF-4 at 7675 seconds. . . . . . . . . . . . . . . . .

4.2.9 Locations of fuel and clad (upper left), particulate debris (upper right), canister-b (lower left) and other structure (lower right) component materials in DF-4 at 7700 seconds. . . . . . . . . . . . . . . . . . . 
4.2.10 Locations of fuel and clad (upper left), particulate debris (upper right), canister-b (lower left) and other structure (lower right) component materials in DF-4 at 8100 seconds. . . . . . . . . . . . . . . .

4.2.11 PIE photo showing final state of the DF. 4 test bundle at the $95 \mathrm{~mm}$ axial level [5]. . . . . . . . . . . . . . . . . .

4.2.12 PIE photo showing final state of the DF-4 test bundle at the $133 \mathrm{~mm}$

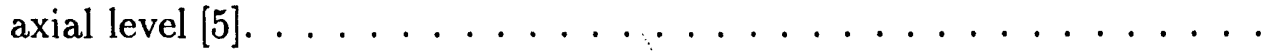

4.2.13 PIE photo showing final state of the DF-4 test bundle at the $238 \mathrm{~mm}$ axial level [5]. . . . . . . . . . . . . . . .

4.2.14 PIE photo showing final state of the DF-4 test bundle at the $308 \mathrm{~mm}$ axial level [5]. . . . . . . . . . . . . . . . . .

4.3.1 Calculated and measured integral hydrogen production in the DF-4 experiment. The final amount of the extrapolated production was given by the PIE. . . . . . . . . . . . . . . . . . . .

4.3.2 Calculated and measured hydrogen production rate in the DF-4 exper-

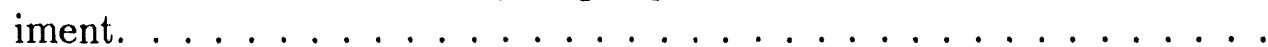

5.1.1 Hydrogen production for variations on the core convective heat transfer coefficients, compared to base case and measured values. . . . . . . . .

5.1.2 Cladding temperatures at the $36.8 \mathrm{~cm}$ plane for variations on the core convective heat transfer coefficients, compared to base case and measured values.

5.1.3 Control blade temperatures at the $25.4 \mathrm{~cm}$ plane for variations on the core convective heat transfer coefficients, compared to base case and measured values. . . . . . . . . . . . . . . . .

5.1.4 Cladding temperatures at the $36.8 \mathrm{~cm}$ plane for the MATPRO sensitivity study, compared to base case and measured values. . . . . . . . . . .

5.1.5 Hydrogen production for the MATPRO sensitivity study, compared to base case and measured values. . . . . . . . . . . . . .

5.1.6 Cladding temperatures at the $25.4 \mathrm{~cm}$ plane for the radiation view factor sensitivity study, compared to base case and measured values. . . . . .

5.1.7 Control blade temperatures at the $36.8 \mathrm{~cm}$ plane for the radiation view factor sensitivity study, compared to base case and measured values. .

5.1.8 Hydrogen production for the radiation view factor sensitivity study, compared to base case and measured values. . . . . . . . . . . .

5.1.9 Cladding temperature response at the $36.8 \mathrm{~cm}$ plane for the canister mass and area partition sensitivity study calculation, compared to base case and measured values. . . . . . . . . . . . . . .

5.1.10 Control blade temperature response at the $25.4 \mathrm{~cm}$ plane for the canister mass and area partition sensitivity study calculation, compared to base case and measured values. . . . . . . . . . . . . . . .

5.1.11 Canister component (top) and canister-b component (bottom) material distributions at 8100 seconds for the canister mass and area partition sensitivity study calculation (base case canister-b component material distributions are shown in Figure 4.2.10). . . . . . . . . . . . 
5.1.12 Hydrogen production for the minimum component mass sensitivity study, compared to base case and measured values. . . . . . . . . . . .

5.1 .13 Cladding temperature response at the $9.6 \mathrm{~cm}$ plane for the candling heat transfer coefficient sensitivity study, compared to base case and measured values. . . . . . . . . . . . . . . . .

5.1.14 Control blade temperature response at the $9.6 \mathrm{~cm}$ plane for the candling heat transfer coefficient sensitivity study, compared to base case and measured values. . . . . . . . . . . . . . . .

5.1.15 Cladding temperature response at the $25.4 \mathrm{~cm}$ plane for the candling heat transfer coefficient sensitivity study, compared to base case and measured values. .................... . .

5.1.16 Control blade temperature response at the $25.4 \mathrm{~cm}$ plane for the candling heat transfer coefficient sensitivity study, compared to base case and measured values. . . . . . . . . . . . . . . . . .

5.1.17 Hydrogen production for the candling heat transfer coefficient sensitivity study, compared to base case and measured values. . . . . . . . . .

5.1.18 Component material distributions at 7600 seconds for the $1 \%$ case of the candling heat transfer coefficient sensitivity study.

5.1.19 Cladding temperatures at the $36.8 \mathrm{~cm}$ plane for the minimum oxide shell thickness sensitivity study, compared to base case and measured values.

5.1.20 Control blade temperatures at the $36.8 \mathrm{~cm}$ plane for the minimum oxide shell thickness sensitivity study, compared to base case and measured values. . . . . . . . . . . . . . . . . . . . .

5.1.21 Hydrogen production for the minimum oxide shell thickness sensitivity study, compared to base case and measured values. . . . . . . . . . .

5.1.22 Component material distributions at 8100 seconds for the $1 \mu \mathrm{m}$ case of the minimum oxide shell thickness sensitivity study. . . . . . . . . . .

5.1.23 CVH bulk atmosphere temperature for the default boundary fluid temperature option sensitivity study, compared to base case values. . . . .

5.1.24 Cladding temperatures at the $36.8 \mathrm{~cm}$ plane for the default boundary fluid temperature option sensitivity study, compared to base case and measured values. . . . . . . . . . . . . . . . . . .

5.1.25 Control blade temperatures at the $36.8 \mathrm{~cm}$ plane for the default boundary fluid temperature option sensitivity study, compared to base case and measured values. . . . . . . . . . . . . . . . . . .

5.1.26 Hydrogen production for the default boundary fluid temperature option sensitivity study, compared to base case and measured values. . . . . .

5.2.1 Hydrogen production for the debris porosity sensitivity study, compared to base case and measured values. . . . . . . . . . . . . . . 101

5.2.2 Particulate debris component material locations at 8100 seconds for the "small" case of the debris porosity sensitivity study. . . . . . . . . 102

5.2.3 Particulate debris component material locations at 8100 seconds for the "large" case of the debris porosity sensitivity study. . . . . . . . . 103

5.2.4 Cladding temperature response at the $9.6 \mathrm{~cm}$ plane for the debris porosity sensitivity study, compared to base case and measured values. . . . 104 
5.2.5 Control blade temperature response at the $9.6 \mathrm{~cm}$ plane for the debris porosity sensitivity study, compared to base case and measured values.

5.2.6 Hydrogen production for the debris diameter sensitivity study, compared to base case and measured values. . . . . . . . . . . . . . . 106

5.2.7 Cladding temperature response at the $9.6 \mathrm{~cm}$ plane for the debris diameter sensitivity study, compared to base case and measured values. . .

5.2.8 Control blade temperature response at the $9.6 \mathrm{~cm}$ plane for the debris diameter sensitivity study, compared to base case and measured values.

5.3.1 Cladding temperature response at the $36.8 \mathrm{~cm}$ plane for the oxidation reaction rate transition temperature sensitivity study, compared to base case and measured values. . . . . . . . . . . . . . . .

5.3.2 Control blade temperature response at the $36.8 \mathrm{~cm}$ plane for the oxidation reaction rate transition temperature sensitivity study, compared to base case and measured values. . . . . . . . . . . . . .

5.3.3 Hydrogen production for the oxidation reaction rate transition temperature sensitivity study, compared to base case and measured values. . .

5.4.1 Other Structure component material locations at 7550 seconds for the eutectic model deactivation sensitivity study. . . . . . . . . . . .

5.4.2 Locations of fuel and clad (upper left), particulate debris (upper right), canister-b (lower left) and other structure (lower right) component materials at 7600 seconds for the eutectic model deactivation sensitivity study. . . . . . . . . . . . . . . . . .

5.4.3 Material locations for the fuel and clad (upper left), particulate debris (upper right), canister-b (lower left) and other structure (lower right) components at 8100 seconds for the eutectic model deactivation sensitivity study. . . . . . . . . . . . . . . . . . . .

5.4.4 Hydrogen production for the eutectic model deactivation sensitivity study, compared to base case and measured values. . . . . . . . . . .

5.4.5 Control blade temperature response at the $36.8 \mathrm{~cm}$ plane for the eutectic model deactivation sensitivity study, compared to base case and measured values. . . . . . . . . . . . . . . . .

5.4.6 Cladding temperature response at the $36.8 \mathrm{~cm}$ plane for the eutectic model deactivation sensitivity study, compared to base case and measured values. . . . . . . . . . . . . . . . . .

5.4.7 Cladding temperature response at the $25.4 \mathrm{~cm}$ plane for the eutectic model deactivation sensitivity study, compared to base case and measured values. . . . . . . . . . . . . . . . . . .

5.4.8 Control blade temperature response at the $36.8 \mathrm{~cm}$ plane for the $\mathrm{B}_{4} \mathrm{C}$ stainless steel eutectic temperature sensitivity study, compared to base case and measured values. . . . . . . . . . . . . . . . .

5.4.9 Control blade temperature response at the $25.4 \mathrm{~cm}$ plane for the $\mathrm{B}_{4} \mathrm{C}$ stainless steel eutectic temperature sensitivity study, compared to base case and measured values. . . . . . . . . . . . . . . .

5.4.10 Hydrogen production for the $\mathrm{B}_{4} \mathrm{C}$-stainless steel eutectic temperature sensitivity study, compared to base case and measured values. . . . . . 
5.4.11 Other Structure component material locations at 7450 seconds for the $1520 \mathrm{~K}$ case of the eutectic temperature sensitivity study. . . . . . . .

5.5.1 Cladding temperature response at the $36.8 \mathrm{~cm}$ plane for the steam mass flow rate sensitivity study, compared to base case and measured values.

5.5.2 Hydrogen production for the steam mass flow rate sensitivity study, compared to base case and measured values. . . . . . . . . . . .

5.5.3 Cladding temperature response at the $36.8 \mathrm{~cm}$ plane for the steam inlet temperature sensitivity study, compared to base case and measured values. . . . . . . . . . . . . . . . . . .

5.5.4 Hydrogen production for the steam inlet temperature sensitivity study, compared tn base case and measured values. . . . . . . . . . .

5.5.5 Cladding temperaiure response at the $36.8 \mathrm{~cm}$ plane for the boundary heat structure conductivity sensitivity study, compared to base case and

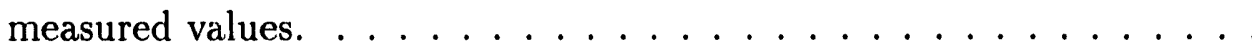

5.5.6 Hydrogen production for the boundary heat structure conductivity sensitivity study, compared to base case and measured values. . . . . . . .

5.5.7 Cladding temperature response at the $36.8 \mathrm{~cm}$ plane for the boundary heat structure temperature sensitivity study, compared to base case and measured values. . . . . . . . . . . . . . . . .

5.5.8 Hydrogen production for the boundary heat structure temperature sensitivity study, compared to base case and measured values. . . . . . . .

5.5.9 Cladding temperature response at the $36.8 \mathrm{~cm}$ plane for the bundle power sensitivity study, compared to base case and measured values.

5.5.10 Hydrogen production for the bundle power sensitivity study, compared to base case and measured values. . . . . . . . . . . . . . .

5.7.1 Cladding temperature response at the $36.8 \mathrm{~cm}$ plane for the COR and CVH nodalization sensitivity study, compared to base case and measured values.

5.7.2 Control blade temperature response at the $36.8 \mathrm{~cm}$ plane for the COR and $\mathrm{CVH}$ nodalization sensitivity study, compared to base case and measured values. $\ldots \ldots \ldots$

5.7.3 Hydrogen production for the bundle power sensitivity study, compared

Time steps used for the time step sensitivity study, compared to base case and measured values. . . . . . . . . . . . . . . .

5.8.2 COR package time steps used for the time step sensitivity study, compared to base case and measured values. . . . . . . . . . . . . .

5.8.3 Cladding temperature response at the $49.5 \mathrm{~cm}$ plane for the time step sensitivity study, compared to base case and measured values. . . . . .

5.8.4 Cladding temperature response at the $36.8 \mathrm{~cm}$ plane for the time step sensitivity study, compared to base case and measured values. . . . . .

5.8.5 Control blade temperature response at the $25.4 \mathrm{~cm}$ plane for the time step sensitivity study, compared to base case and measured values. . .

5.8.6 Hydrogen production for the time step sensitivity study, compared to base case and measured values. . . . . . . . . . . . . . 150 
5.8.7 Cladding temperatures at the $36.8 \mathrm{~cm}$ plane for the six computers in the machine dependency sensitivity study, compared to measured values. .

5.8.8 Control blade temperatures at the $36.8 \mathrm{~cm}$ plane for six computers in the machine dependency sensitivity study, compared to measured values. . . . . . . . . . . . . . . . . . .

5.8.9 Hydrogen production for six computers in the machine dependency sensitivity study, compared to measured values. . . . . . . . . . .

5.8.10 Component material locations at 8100 seconds for the Cray X/MP-24 case of the machine dependency sensitivity study. For an explanation of the format of this figure, see Section 4.2.1. . . . . . . . . . . . .

5.8.11 Component material locations at 8100 seconds for the DECstation $5000 / 240$ case of the machine dependency sensitivity study. For an explanation of the format of this figure, see Section 4.2.1 . . . . . . . 156

5.8.12 Component material locations at 8100 seconds for the Gateway 486/DX250 case of the machine dependency sensitivity study. For an explanation of the format of this figure, see Section 4.2.1.

5.8.13 Component material locations at 8100 seconds for the Hewlett Packard 755 case of the machine dependency sensitivity study. For an explanation of the format of this figure, see Section 4.2.1. . . . . . . . . .

5.8.14 Component material locations at 8100 seconds for the IBM RS/6000 Model 550 case of the machine dependency sensitivity study. For an explanation of the format of this figure, see Section 4.2.1. . . . . . . .

5.8.15 Component material locations at 8100 seconds for the SUN Sparcstation 2 case of the machine dependency sensitivity study. For an explanation of the format of this figure, see Section 4.2.1.

5.8.16 System time steps used by the six computers in the machine dependency sensitivity study.

5.8.17 COR package time steps used by the six computers in the machine dependency sensitivity study. . . . . . . . . . . . . .

5.8.18 Overall execution time used by the six computers in the machine depen-

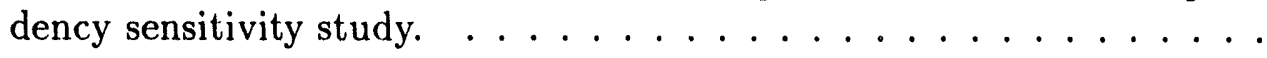
162

5.8.19 Package execution times for the DF-4 base case model executed on a DECStation $5000 / 240 \ldots \ldots \ldots \ldots$. . . . . . . . . . . 164

6.1.1 Comparison of control blade temperatures at the $36.8 \mathrm{~cm}$ plane calculated by APRIL, BWRSAR/DF4, MELPROG, SCDAP/RELAP, and MELCOR against experimental results.

6.1.2 Comparison of control blade temperatures at the $25.4 \mathrm{~cm}$ plane calculated by APRIL, BWRSAR/DF4, MELPROG, SCDAP/RELAP, and MELCOR against experimental results. . . . . . . . . . . .

6.1.3 Comparison of cladding temperatures at the $36.8 \mathrm{~cm}$ plane calculated by APRIL, BWRSAR/DF4, MELPROG, SCI)AP/RELAP, and MELCOR against experimental results. . . . . . . . . . . . 
6.1.4 Comparison of cladding temperatures at the $25.4 \mathrm{~cm}$ plane calculated by APRIL, BWRSAR/DF4, MELPROG, SCDAP/RELAP, and MELCOR against experimental results. . . . . . . . . . . . . 171

6.1.5 Comparison of cladding temperatures at the $49.5 \mathrm{~cm}$ plane calculated by APRIL, BWRSAR/DF4, MELPROG, SCDAP/RELAP, and MELCOR against experimental results. . . . . . . . . . . . . 172

6.2.1 Comparison of the hydrogen generation rates calculated by APRIL, BWRSAR/DF4, MELPROG, SCDAP/RELAP, and MELCOR against experimental results. . . . . . . . . . . . . . . 


\section{List of Tables}

2.1.1 Important parameters for the DF-4 experiment [6]. Core material masses are for active fuel region only. . . . . . . . . . . . . . 6

3.1.1 View factors used in MELCOR DF-4 base case model, compared to analytical values given in $[8] . \ldots \ldots \ldots 14$

3.1.2 Core radial boundary heat structures parameters. . . . . . . . . . . 17

3.2.1 Initial conditions for the MELCOR base case input model for the DF-4

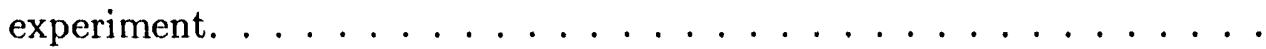

3.2.2 Starting elevations and power peaking factors for core cells in the MELCOR DF-4 base case in put model. Axial levels in the active fuel regions are $5.6 \mathrm{~cm}$ in height. . . . . . . . . . . . .

5.8.1 CVH and COR subcycle ratios used by the six computers in the machine dependency sensitivity study. Also shown are the total execution times and the total execution times normalized to that of the Hewlett Packard 755

6.2.1 Comparison of the total hydrogen generation calculated by MELCOR, APRIL, MELPROG and SCDAP/RELAP5 to experimental data. Experimental data is from the PIE [5], which gave a range of possible

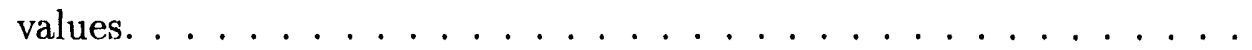




\section{Acknowledgements}

I wish to acknowledge the many contributions made to this assessment task by L. N. Kmetyk. Luba was involved in this task from start to finish, contributing her expertise and expert opinions to help develop a reasonable base case MELCOR model and meaningful sensitivity studies. She also was infinitely patient in reviewing the many drafts of this report. The software used to generate core material location plots originated from L. N. Kmetyk and S. L. Thompson. I would also like to acknowledge Rod Schmidt, who contributed to the early stages of this task, helping me to understand the important features of the DF-4 experiment and the MELPROG representation of it. Finally, I would like to acknowledge the support and encouragement of the MELCOR development group at Sandia National Laboratories.

This work was funded by the Office of Accident Evaluation, U. S. Nuclear Regulatory Commission. 
$$
\underline{ }
$$

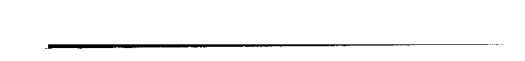

$\mid$

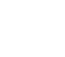

s.

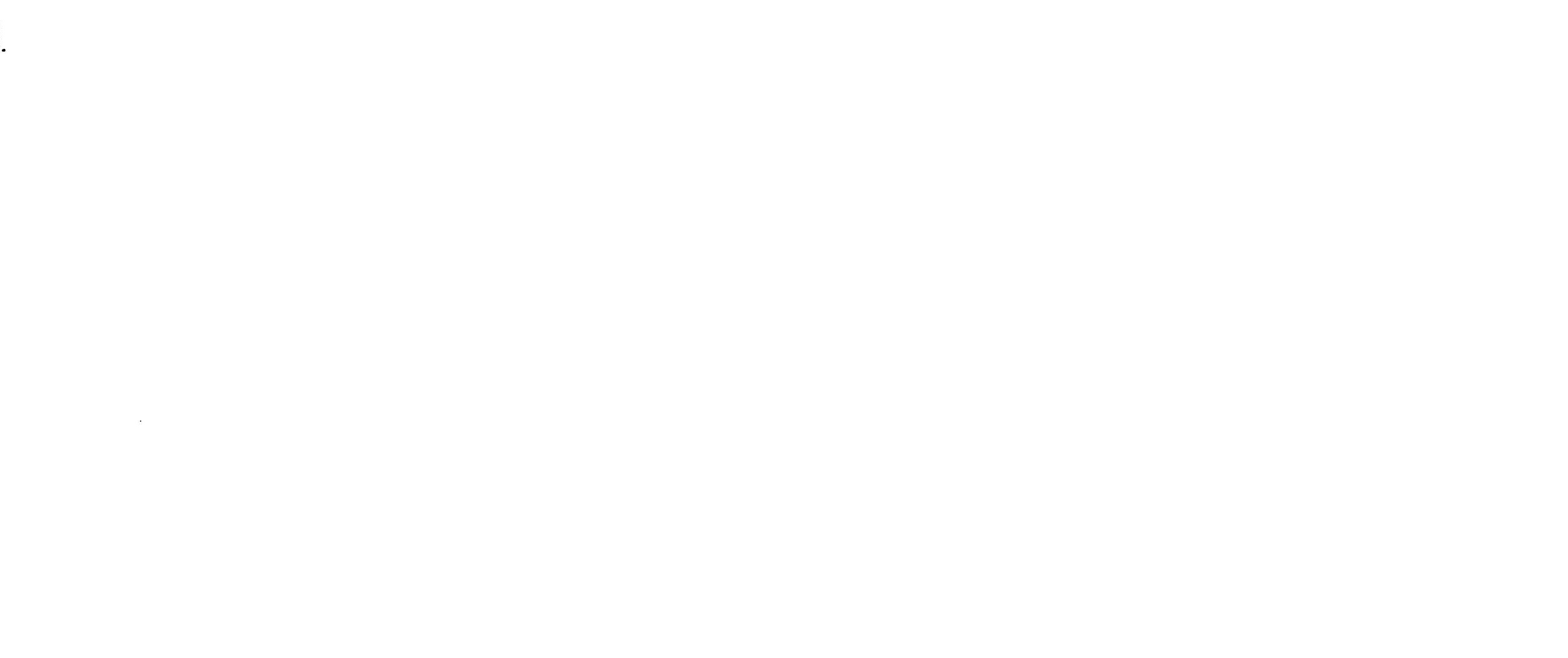




\section{Chapter 1}

\section{Introduction}

MELCOR is a fully integrated, engineering-level computer code being developed at Sandia National Laboratories for the U. S. Nuclear Regulatory Commission (US$\mathrm{NRC}$ ), that models the progression of severe accidents in light water reactor nuclear 1 wer plants [1]. The entire spectrum of severe accident phenomena, including reactor coolant system and containment thermal/hydraulic response, core heatup, degradation and relocation, and fission product release and transport, is treated in MELCOR in a unified framework for both boiling water reactors and pressurized water reactors.

The purpose of the Damaged Fuel, or DF, series of experiments [2],[3],[4],[5] was to investigate core melt progression and to benchmark severe accident codes. The DF-4 experiment was carried out at Sandia National Laboratories in the Annular Core Research Reactor (ACRR) in 1986 [6]. This experiment investigated the behavior of BWR-type fuel materials and configurations in a high-temperature oxidizing environment typical of the conditions during a Loss-Of-Coolant Accident (LOCA). The DF-4 experiment provided information about the physical processes which are important during the early fuel damage phase of a severe accident in a BWR. This experiment provided detailed measurements of experimental conditions in the test bundle, against which MELCOR and other severe accident codes can be assessed.

The primary areas of interest in the DF-4 experiment were the eutectic interaction between the control poison material $\left(\mathrm{B}_{4} \mathrm{C}\right)$ and the stainless steel control blade sheath and tubes, and the oxidation of zircaloy in the cladding and canister. The eutectic interaction was important because it contributed to early control blade failure; this could result in reactivity transients if the control poison were to relocate independently of the fuel.

For this task, an assessment of the MELCOR code was performed using the DF-4 experiment. With the recent addition of eutectic interaction modeling [7], MELCOR is able to model most relevant phenomena in DF-4. These calculations were performed as post-test analyses. with both test data and the results of other code analyses available for comparison. Sensitivity studies were performed on the core (COR) package, control volume hydrodynamics $(\mathrm{CVH})$ package and heat structures (HS) package parameters, and the results of the base case calculation are compared with results from other codes modeling DF-4. The version of MELCOR used to model 
DF-4 was version $1.8 \mathrm{NX}$ of the code. All modifications resulting from this assessment task have been implemented in the production version of the code.

The remainder of this report is structured as follows. The experimental setup of DF-4 is described in Chapter 2. The base case MELCOR model for DF-4, which represents the best approximation of experimental conditions, is described in Chapter 3. The results calculated with MELCOR using the base case input model are discussed in Chapter 4. A number of sensitivity studies were done on MELCOR parameters in the COR, CVH and HS packages, on the input problem nodalization, and on the time steps used to model DF-4. These studies are discussed in Chapter 5. The MELCOR base case results are compared with results from four other severe accident codes in Chapter 6. Several code problems were identified as part of this work; these problems and the corrections made to MELCOR to fix them are described in Chapter 7. This work is summarized and final conclusions are given in Chapter 8. The MELCOR input deck used for the base case calculation is given in Appendix A. 


\section{Chapter 2}

\section{Facility and Test Description}

The DF-4 experiment [5] was part of a series of Damaged Fuel experiments conducted at Sandia National Labs between 1984 and 1986 [2],[3],[4],[5], using the ACRR at Sandia to provide the nuclear heating which drove the bundle damage progression. The DF experiments involved the nuclear heating of a representative fuel bundle to the point of core material melting, oxidation and relocation.

\subsection{Facility Description}

The DF-4 experiment represented a typical BWR D-Lattice core geometry. The fuel bundle in DF-4 consisted of fuel rods, a canister or channel box, and a control blade sheath surrounding five steel-clad $\mathrm{B}_{4} \mathrm{C}$ control rods. These components were surrounded by several layers of thermal insulation. A radial cross section of the DF-4 fuel bundle is shown in Figure 2.1.1. Each of these components is described in the following sections, and general core component parameters are given in Table 2.1.1 (for more details of the experimental facility, see [5]).

\subsubsection{Stainless Steel/B $\mathbf{B}_{4}$ C Control Blade}

Located at the center of the DF-4 test bundle was the control blade, consisting of five stainless steel tubes filled with $\mathrm{B}_{4} \mathrm{C}$ powder ( $70 \%$ theoretical density), surrounded by a stainless steel sheath. Dimensions for the control blade tubes and sheath are typical of a General Electric D-Lattice design (see Figure 2.1.1 and Table 2.1.1).

\subsubsection{Channel Box}

The control blade was completely surrounded by a zircaloy- 4 channel box or canister. This arrangement was opposite that of a normal BWR fuel assembly, where the channel box surrounds the fuel rods and not the control blade. This arrangement still allowed separate steam flow regions for the fuel rods and control blade, as occurs in normal BWR cores. The thickness of the canister walls and the gap thickness between the sheath and the inside canister wall were typical of the GE D-Lattice design. The control blade was displaced to one end inside the canister (see Figure 2.1.1) to 


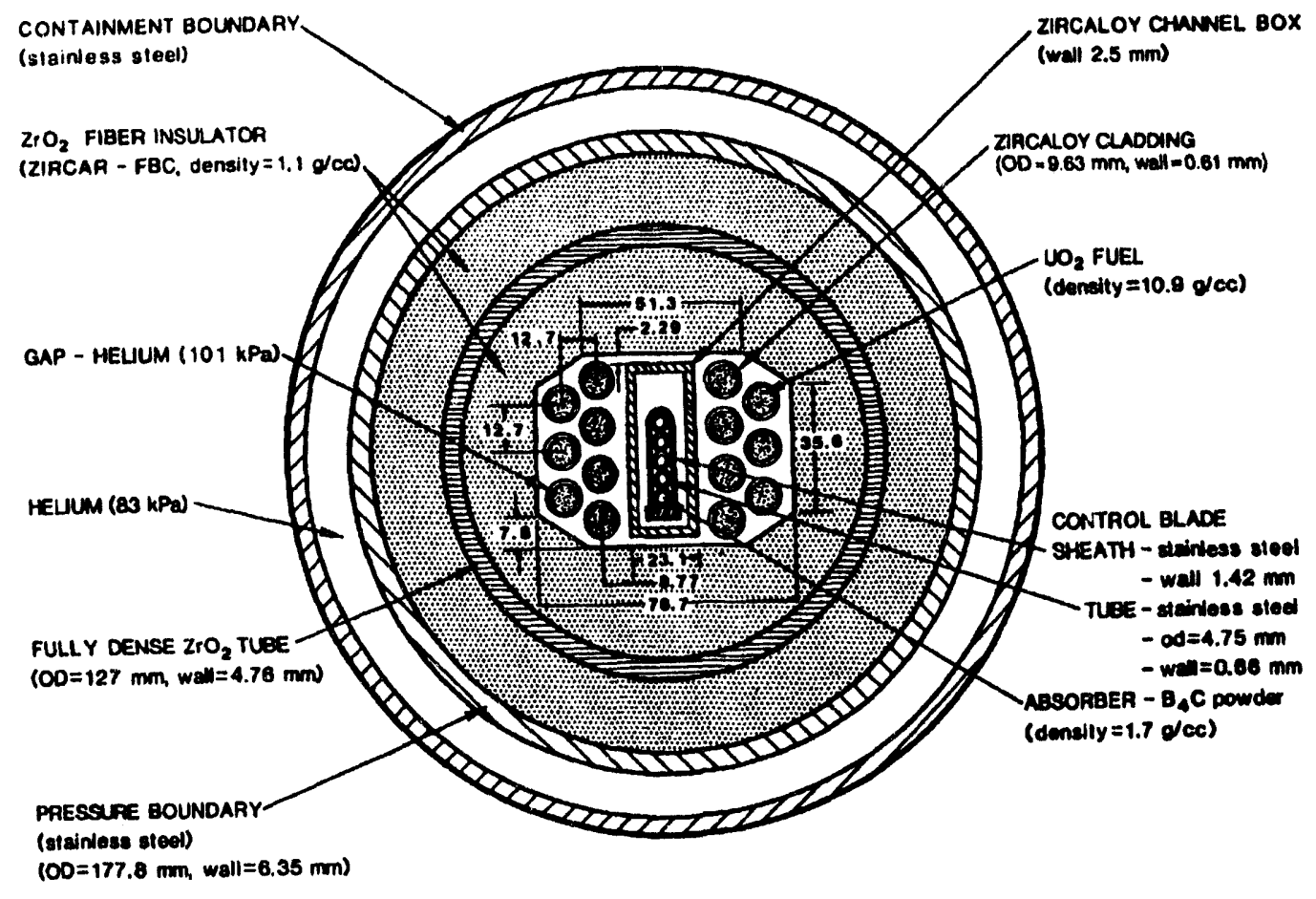

Figure 2.1.1. Radial cross section of the DF-4 test bundle (from [6]). 


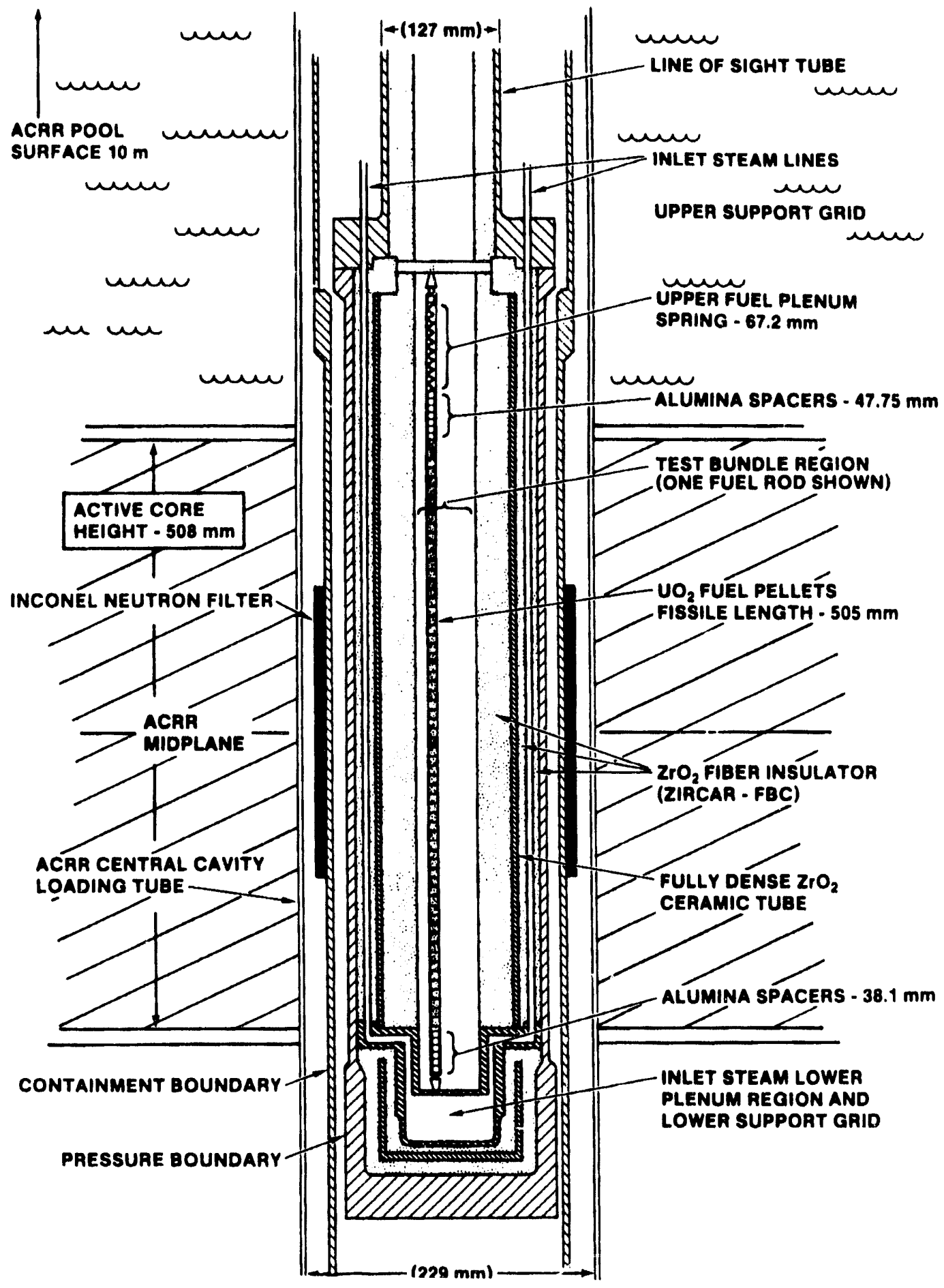

Figure 2.1.2. Axial cross section of the DF-4 test bundle (from [6]). 
Table 2.1.1. Important parameters for the DF-4 experiment [6]. Core material masses are for active fuel region only.

\begin{tabular}{c|c} 
Parameter & Value \\
\hline Dimensions: & \\
Fuel pellet diameter & $8.26 \mathrm{~mm}$ \\
Cladding thickness & $0.61 \mathrm{~mm}$ \\
Fuel rod OD & $9.63 \mathrm{~mm}$ \\
Channel box thickness & $2.5 \mathrm{~mm}$ \\
Fuel rod pitch & \\
(square \& triangular) & $12.7 \mathrm{~mm}$ \\
& \\
Masses: & $4.13 \mathrm{~kg}$ \\
Fuel & $0.79 \mathrm{~kg}$ \\
Zircaloy (clad) & $1.08 \mathrm{~kg}$ \\
Zircaloy (canister) & $0.57 \mathrm{~kg}$ \\
Stainless steel & $0.04 \mathrm{~kg}$ \\
Boron carbide &
\end{tabular}

represent the tip region of a typical BWR control blade. Various canister dimensions are given in Table 2.1.1.

\subsubsection{Fuel Rods}

Seven fuel rods were situated on each side of the canister in the DF-4 test bundle, for a total of 14 rods. The rods on each side of the canister were arranged in two rows, with the inner row in a square $1.3 \mathrm{~cm}(0.5 \mathrm{in}$.) pitch and the outer row in a triangular $1.3 \mathrm{~cm}(0.5 \mathrm{in}$.) pitch (see Figure 2.1.1). This allowed increased radiation view factors between the outer row of fuel rods and the canister while decreasing the overall size of the test bundle.

The fuel rods consisted of $10 \%$ enriched $\mathrm{UO}_{2}$, clad by zircaloy- 4 . The fuel rods were more prototypic of PWli wh (which were more readily available), but their pitch and the distance to the channel box outer wall were typical of BWR fuel assemblies. The active fuel region was $50.5 \mathrm{~cm}$ long. The end regions consisted of $\mathrm{Al}_{2} \mathrm{O}_{3}$ spacers and zircaloy-4 end caps, with an Inconel spring on the upper end to hold the fuel pellets in place. The fuel rods were backfilled with helium gas to approximately atmospheric pressure. An axial cross section of the DF-4 test bundle is shown in Figure 2.1.2. 


\subsubsection{Test Section Insulation and Radial Boundary}

The active test bundle, consisting of fuel rods, a control blade and a canister, was surrounded by four layers of insulation. These layers served as thermal and material boundaries for the test bundle.

The first layer of insulation, closest to the fuel rods, consisted of a porous zirconia $\left(\mathrm{ZrO}_{2}\right)$ material conformed to the general shape of the active test bundle. This material formed the primary thermal barrier to the test bundle, and had an outer diameter of $12.2 \mathrm{~cm}$. The second layer consisted of dense $\mathrm{ZrO}_{2}$, which acted as a barrier to any molten core material penetrating the inner porous zirconia layer. Porous zirconia formed the third layer, with an outer diameter of about $17 \mathrm{~cm}$. The fourth layer of insulation was a $6 \mathrm{~mm}$ thick stainless steel wall, which formed the pressure boundary to the test bundle.

\subsubsection{Other DF-4 Experiment Components}

There were several components outside the DF-4 test bundle that were important to the execution of the experiment. These components are described individually.

\section{Steam Supply System}

The steam line system was used to provide steam to the test bundle. Two inlet steam lines entered the test bundle from the top, and ran down between the dense zirconia tube and the stainless steel pressure boundary. These lines emptied into a lower plenum below the test bundle, from which the steam could flow into the fuel (channel) and control blade (bypass) regions.

The stainless steel plate separating the inlet plenum and the channel and bypass regions of the test bundle (see Figure 2.1.2) contained orifices, one each to the channel and bypass regions, such that $80 \%$ of the flow entered the channel region ( $40 \%$ to each side of the canister) and $20 \%$ entered the bypass region. This flow split was maintained throughout the experiment by a large pressure drop across the orifices, so that the flow was not influenced by downstream pressure.

\section{Test Bundle Instrumentation}

The primary instrumentation in the test bundle consisted of temperature thermocouples. These thermocouples were located at four axial levels in the active fuel region, and were physically attached to the fuel rod clad, control blade sheath and canister walls. Two types of thermocouples were used in the DF-4 experiment. The first type (designated "type $\mathrm{S}$ ") was a platinum-rhodium $(\mathrm{Pt} / \mathrm{Rh})$ thermocouple, welded directly to the instrumented component. This type had good response time, but failed at the substrate melting temperature (which was $1700 \mathrm{~K}$ for the stainless steel control blade and $2200 \mathrm{~K}$ for the zircaloy cladding). "Type C" thermocouples were fabricated from tungsten-rhenium (W/Re), and were sheathed in a ceramic zirconia tube. This sheath touched the component being instrumented. Because of the sheath and also because of the horizontal placement of these thermocouples (see Figure 2.1.3 and 
[5]), the temperature indicated by these thermocouples lagged the actual component temperatures by several huldred degrees $\mathrm{K}$. However, these instruments survived component melting because of the zirconia sheath.

The axial locations of both types of thermocouples are shown in Figure 2.1.3. Type $\mathrm{S}$ thermocouples are indicated with a black dot on the component instrumented, while type $\mathrm{C}$ thermocouples are indicated with a sheath assembly that touches the instrumented component. Distances in Figure 2.1.3 are measured from the bottom of the active fuel region (see Figure 3.1.2 for the relative axial positioning within the assembly). Also shown are the alphanumeric indicators for each thermocouple. The notation used for these alphanumeric indicators is explained in Figure 2.1.4.

\section{Hydrogen Recombiner}

The system used to measure hydrogen production in DF-4, referred to as the hydrogen recombiner, was designed specially for this experiment. It measured the heat liberated from a chemical reaction between $\mathrm{CuO}$ and $\mathrm{H}_{2}$, and from that inferred the mass of $\mathrm{H}_{2}$ flowing through the recombiner. A measure of the integral amount of hydrogen produced during DF-4 was provided by subsequent PIE's (Post Irradiation Examinations) performed by the experimenters [6].

\section{Visual Diagnostic System}

The upper portion of the test bundle was kept clear of structure and components to allow visual observation of experiment progression. Steam exiting from the test bundle was diverted to an outlet line using an opposing steam flow [5]. Video pictures were taken through an optical port in the top of the experiment package. This visual record provided further data on the timing of major experimental phenomena, such as control blade failure. 

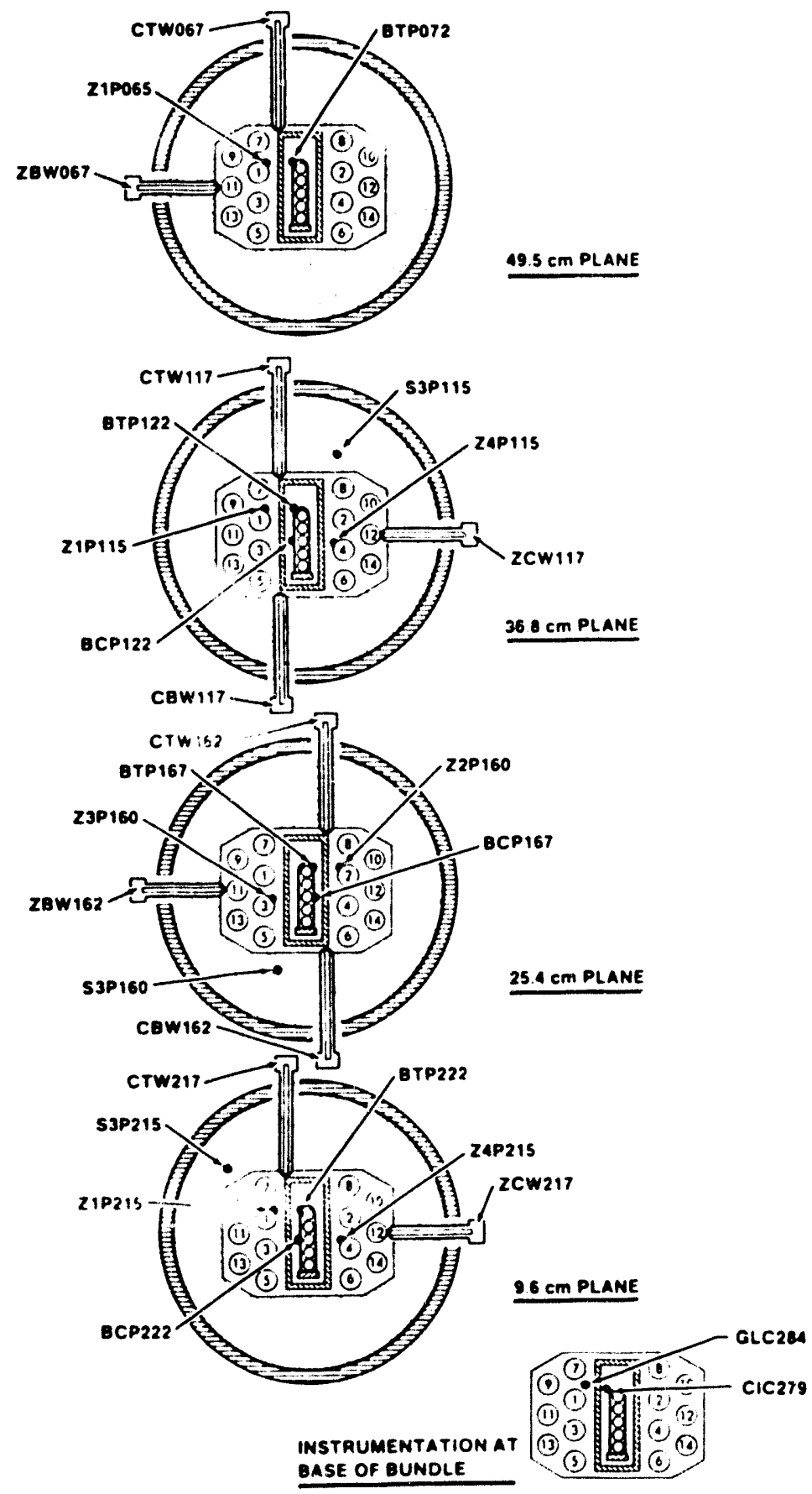

Figure 2.1.3. Radial locations of bundle thermocouples (from [6]). 

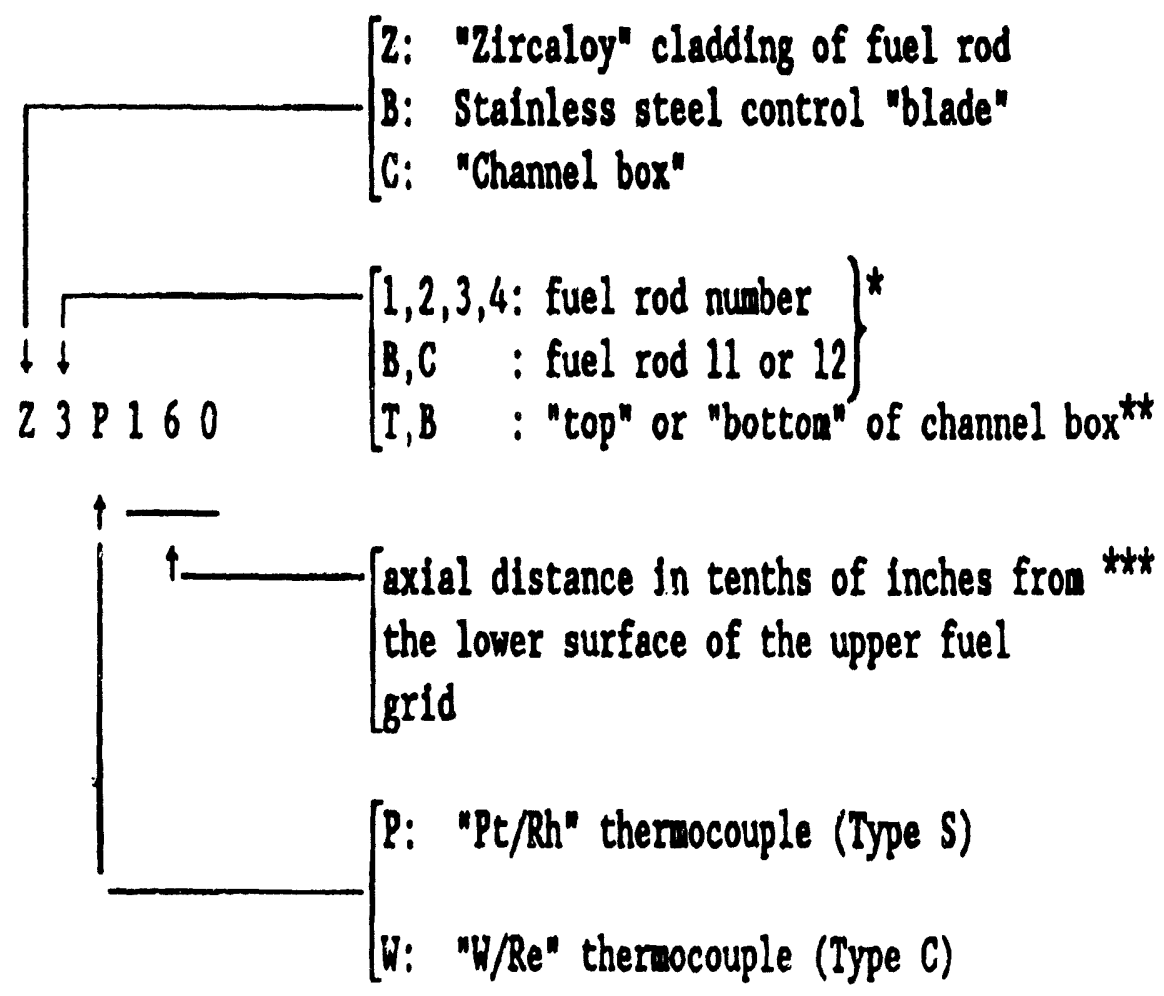

- - this notation used only if the leading prefix is "Z" for zircaloy fuel rod cladding

" - this notation used when the leading prefix is "C" for channel box

"'. - this datum is located 26 inches $(660 \mathrm{~mm})$ above the bottom of the fuel rod fissile lengh.

Figure 2.1.4. Mnemonic indicators for bundle thermocouple locations (from [6]). 


\subsection{Test Description}

The DF-4 test consisted of three primary phases: the startup phase $(0-2200$ seconds), the hydrogen calibration phase (2200-6000 seconds), and the fuel damage phase (6000-8100 seconds). The purposes of the first two phases were to bring the test bundle to the proper thermal initial conditions, and to calibrate the hydrogen recombiner system. These two phases were not modeled by MELCOR and therefore are not described in this report. The results of these two phases are represented by the MELCOR input model in the initial conditions of the test bundle. The remaining phase and its "subphases" can be represented best by a plot of the ACRR reactor power versus time, shown in Figure 2.2.1, and the inlet steam mass flow rate, shown in Figure 2.2.2. (Note that during the entire test, the inlet steam was partitioned such that $80 \%$ went to the fuel rods outside the channel box and $20 \%$ was diverted to the control blade inside the channel box.)

The fuel damage phase extended over the period of 6000-8100 seconds. Although five separate subphases were defined [5], the most important of the subphases were the oxidation pre-transient subphase (7200-7500 seconds) and the oxidation transient subphase (7500-8000) seconds. It was during these subphases that most of the test bundle damage occurred. During the other subphases, the test bundle components underwent nuclear heating and reached approximate temperature equilibrium.

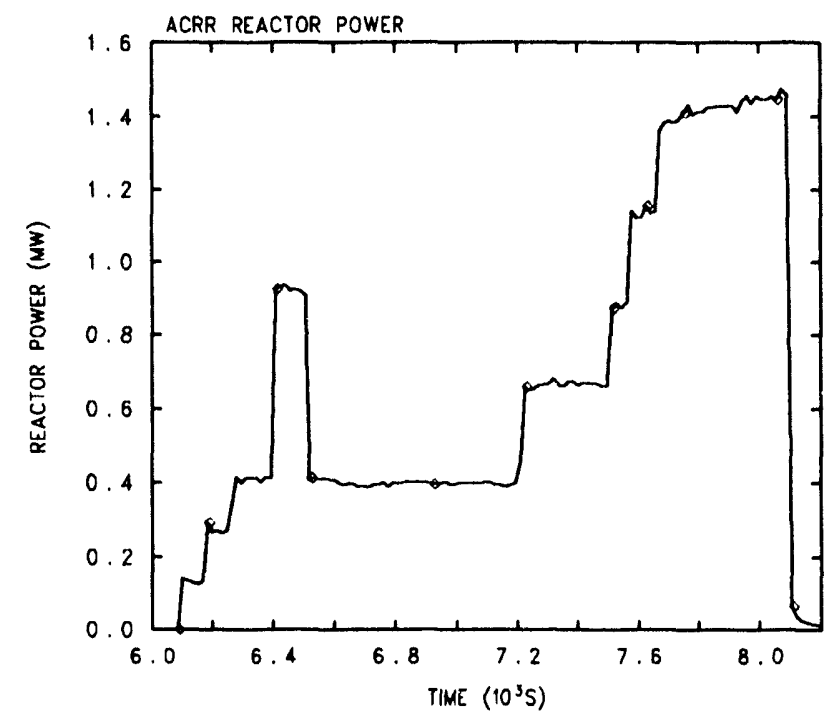

Figure 2.2.1. Measured reactor power in the ACRR during the fuel damage phase of the DF-4 experiment. 


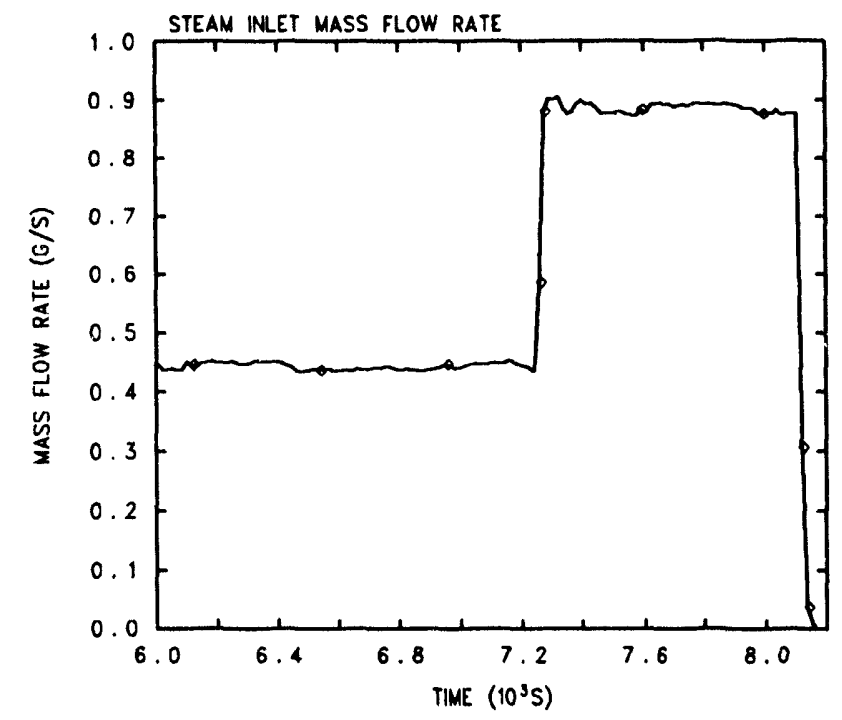

Figure 2.2.2. Total inlet steam flow rate during the fuel damage phase of the DF-4 experiment. 


\section{Chapter 3}

\section{MELCOR Input Model}

The input model used to represent the DF-4 experiment for the base case calculation is described in this chapter. The base case model did not use all the MELCOR default input options, but rather used input which resulted in best agreement with experimental data. This base case was derived from a number of sensitivity studies, some of which are described in Chapter 5.

The part of the MELCOR base case model describing geometric and mass input is described first, followed by a discussion of initial and boundary conditions used in the base case calculation. A listing of the base case MELCOR input is included in Appendix A, for reference.

\subsection{Geometry}

The DF- 4 base case input model represented only those parts of the DF- 4 experiment that were considered significant to the results of the experiment. The parts of the experiment modeled included the active test bundle (fuel rods, canister, control blade), the lower or inlet plenum, and the test bundle insulation. Any experimental conditions outside these boundaries were modeled in MELCOR as boundary conditions.

\subsubsection{CVH/FL Package Models}

The base case MELCOR model consisted of four fluid flow control volumes and four flow path junctions. The arrangement of the control volumes and flow paths is shown in Figure 3.1.1. The two control volumes numbered CV101 and CV102 corresponded to the core channel and bypass volumes, respectively. The channel volume included the inlet plenum, while the bypass volume started at the bottom of the fuel rods. A time-specified control volume, CV100, was used to provide the inlet mass flow to the channel and bypass volumes. A sensitivity study on the number of control volumes in the test bundle region is described in Section 5.7.

The flow paths connecting the inlet volume to the channel and bypass volumes were numbered FL101 and FL102, respectively. The flow areas in these flow paths were determined by the cross-sectional flow areas of the channel and bypass regions, 
Table 3.1.1. View factors used in MELCOR DF-4 base case model, compared to analytical values given in [8].

\begin{tabular}{l|c|c}
\multicolumn{1}{c|}{ View Factor } & MELCOR Value & Analytical Value \\
\hline $\mathrm{F}_{\text {can-clad }}$ & 0.550 & 0.5836 \\
$\mathrm{~F}_{\text {blade-can }}$ & 0.9999 & 0.9999 \\
$\mathrm{~F}_{\text {cell-cell(radial) }}$ & 0.10 & 0.0818 \\
$\mathrm{~F}_{\text {cell-cell(axial) }}$ & 0.50 & $\mathrm{~N} / \mathrm{A}$
\end{tabular}

respectively, in the test bundle. Since the channel and bypass control volumes included the entire axial extent of the test bundle, the lengths of both inlet flow paths were set to half the distance between the lower plenum and the end of the test bundle. The outlet flow paths, FL201 and FL202, were similar to the inlet flow paths, and started at the middle of the test bundle.

\subsubsection{COR Package Model}

The core package model used for the base case calculation consisted of one radial ring and fourteen axial levels, for a total of fourteen core cells. Nine of these cells represented active fuel cells, and were numbered cells 104-112. The remaining five cells represented the inlet plenum (cell 101), the core plate region (cell 102), the lower and upper spacer regions (cells 103 and 113, respectively) and the upper spring region (cell 114). The core nodalization used in the DF-4 input model is shown in Figure 3.1.2. A core nodalization study is described in Section 5.7.

The materials used for the active fuel, clad, canister, control blade sheath and tube, and control poison materials corresponded exactly to the materials used in the experiment. Since the MELCOR code does not allow assignment of fuel material on a cell-by-cell basis, the spacer material in the DF-4 fuel rods was specified to be $\mathrm{UO}_{2}$, like the active fuel (the actual material used was $\mathrm{Al}_{2} \mathrm{O}_{3}$ ). Likewise, the Inconel spring in the upper-most core cell was approximated as stainless steel in the other structure component. Zero peaking factors were used in the spacer regions (core cells 103 and 113), resulting in no power generation in those regions (peaking factor input is described in Section 3.2).

The radiation view factors used in the base case MELCOR model are listed in Table 3.1.1, along with the values calculated analytically for the DF-4 experiment [8]. A sensitivity study on the radiation view factors is discussed in section 5.1. 4 .

The minimum component mass and the minimum mass used for temperature control in sensitivity coefficient array 1502 were set to $10^{-5}$ and $10^{-4} \mathrm{~kg}$, respectively. These are the component masses below which component mass and energy are discarded and below which a component is not subject to maximum temperature change criteria, respectively. These parameters were reduced to take into account the reduced component masses in this experiment compared to those found in full-scale reactor core models. A sensitivity study on these parameters is described in Section 5.1.6. 


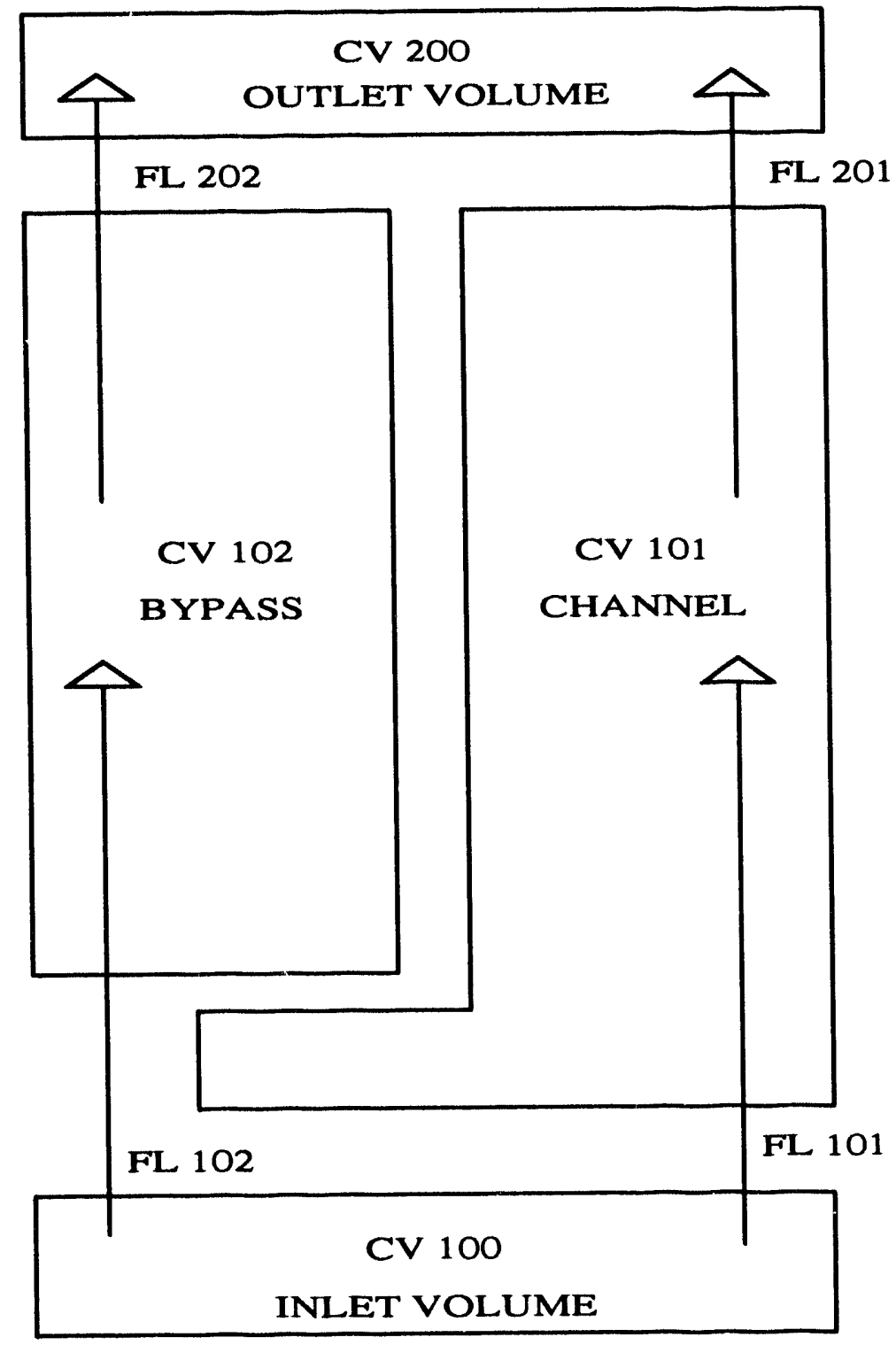

Figure 3.1.1. Control volume and flow path arrangement in the MELCOR DF-4 base case input model. 


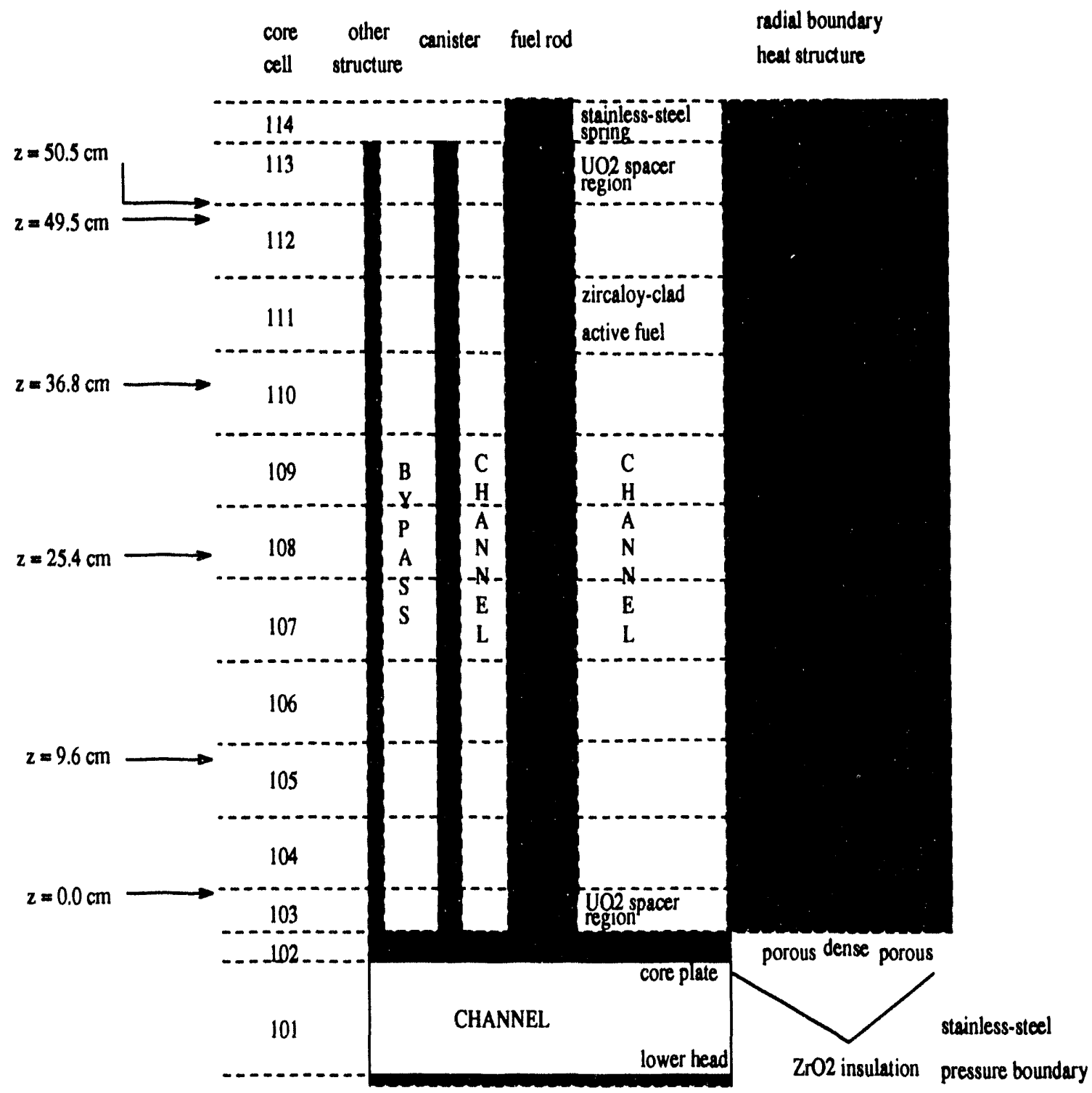

Figure 3.1.2. Core and heat structures nodalization in the MELCOR DF-4 base case input model (upper boundary heat structure not shown). 
Table 3.1.2. Core radial boundary heat structures parameters.

\begin{tabular}{c|c|c|c} 
Layer & Material & Thickness & Number of temperature nodes \\
\hline 1 & $\mathrm{ZrO}_{2}$ (porous) & $2.43 \mathrm{~cm}$ & 11 \\
2 & $\mathrm{ZrO}_{2}$ (dense) & $0.48 \mathrm{~cm}$ & 10 \\
3 & $\mathrm{ZrO}_{2}$ (porous) & $1.91 \mathrm{~cm}$ & 10 \\
4 & Stainless steel & $0.64 \mathrm{~cm}$ & 5
\end{tabular}

The minimum thicknesses for $\mathrm{ZrO}_{2}$ and stainless steel oxide required to hold up molten material, in SC1131, were set to one tenth the clad and other structure thicknesses, respectively. A sensitivity study on these parameters is discussed in Section 5.1.8.

The sensitivity coefficients in SC1501 were adjusted such that all of the input canister mass and area were allocated to the "canister- $b$ " component (that part of the canister that is adjacent to the control blade). This took into account the geometry in the DF-4 test bundle, where most of the canister surface area was adjacent to the fuel rods (see Figure 2.1.1). A sensitivity study on this parameter is discussed in Section 5.1.5.

The temperature used to model the eutectic interaction between $\mathrm{B}_{4} \mathrm{C}$ and stainless steel was changed in $\mathrm{SC}: 1011 \mathrm{f}_{\mathrm{i}} \mathrm{um} 1520 \mathrm{~K}$ to $1570 \mathrm{~K}$. A sensitivity study on this parameter is described in Section 5.4.2. The $\mathrm{B}_{4} \mathrm{C}$-stainless steel eutectic interaction temperature was an important factor for this experiment in predicting the timing of control blade failure and relocation.

The properties of zircaloy were changed to the values given by the MATPRO material properties package [9]. In particular, the melting temperature of zircaloy was changed from $2098 \mathrm{~K}$ to $2200 \mathrm{~K}$. Other MELCOR assessments have shown the MATPRO value to be superior [10], [11]. A sensitivity study on these properties is discussed in Section 5.1.2.

The density of the control poison material, $\mathrm{B}_{4} \mathrm{C}$, was adjusted using MP package input. The density used in the MELCOR base case model was $1700.0 \mathrm{~kg} / \mathrm{m}^{3}$, and accounted for the $70 \%$ packing density for $\mathrm{B}_{4} \mathrm{C}$ given in [5].

\subsubsection{Heat Structures Model}

The base case MELCOR model for DF-4 contained fifteen heat structures. Fourteen of these were radial boundary heat structures, one for each core axial level, required by the COR package. The last heat structure was the upper axial boundary heat structure. The radial heat structures consisted of four material layers; from the inside out, they were: porous zirconia, dense zirconia, porous zirconia, and stainless steel. The thickness of these layers and the number of nodes used to model them are given in Table 3.1.2 (all heat structure nodes are equally spaced). The materials that made up the radial heat structures in the MELCOR base case model are shown in Figure 3.1.2. 
The porous zirconia material $\left(\mathrm{ZrO}_{2}, 77 \%\right.$ porosity) used in the radial boundary heat structure was input to the MP package as a new material. Its density was $23 \%$ of the MELCOR-default value for $\mathrm{ZrO}_{2}$. The specific enthalpy and specific heat tables were not changed, since these quantities are scaled by mass. The thermal conductivity for this material was represented by an empirical correlation given in [8]:

$$
k_{\text {effective }}=0.11498+0.795289 \cdot 10^{-4} T+0.155981 \cdot 10^{-6} T^{2} \mathrm{~W} /(\mathrm{m}-\mathrm{K}) .
$$

This correlation took into account the augmentation of heat transfer at high temperatures by radiation and the penetration of the porous $\mathrm{ZrO}_{2}$ by steam. The expression in Equation (3.1) is compared to the thermal conductivity for $\mathrm{ZrO}_{2}$ (taken from the MELCOR default material properties database and scaled by a factor of 0.23 to take into account the reduced density) in Figure 3.2.1. The increasing slope of the correlation curve with increasing temperature shows that radiation heat transfer is taken into account at higher temperatures. A sensitivity study on the thermal conductivity used for porous $\mathrm{ZrO}_{2}$ is discussed in Section 5.5.3.

The material properties for the dense zirconia layer were the default MELCOR values for $\mathrm{ZrO}_{2}$.

All heat structures in the DF-4 MELCOR input model used the "equiv-band", or equivalent band, radiation model, with emissivities of 0.7 . A sensitivity study on the HS package radiation model option is described in Section 5.6.1, and a sensitivity study on the emissivities used for all heat structures surfaces is described in Section 5.6.2.

The inner boundaries of the radial boundary heat structures were specified to communicate with fluid temperatures calculated by the COR package rather than bulk control volume temperatures ${ }^{1}$. This capability was added as a result of this project (see Section 7 for a complete description of the Defect Investigation Reports (DIRs) resulting from this project).

The boundary conditions used on either side of the fifteen heat structures are discussed in Section 3.2.

\subsection{Test Bundle Initial and Boundary Conditions}

Although the DF-4 experiment had a duration of over 8000 seconds, the first 6000 seconds were used to calibrate instrumentation and establish bundle initial conditions. Therefore the MELCOR analyses had a starting time of 6000 seconds, with the initial conditions specified to match bundle conditions at that time. Also, the test bundle was a small part of the overall DF-4 experiment, which included hydrogen recombiners, a steam condenser, and other equipment. Since the test bundle was of primary interest, the operation of the other experimental components was specified in

\footnotetext{
${ }^{1}$ Fluid temperatures calculated by the COR package $\mathrm{dT} / \mathrm{dz}$ model have a finer resolution than control volume bulk temperatures, which apply over many core cells
} 
Table 3.2.1. Initial conditions for the MELCOR base case input model for the DF-4 experiment.

\begin{tabular}{l|c}
\multicolumn{1}{c|}{ Property } & Value \\
\hline Initial Core Component Temperatures & $480 \mathrm{~K}$ \\
Channel \& Bypass Volume Temperatures & $480 \mathrm{~K}$ \\
Channel \& Bypass Volume Pressures & $0.716 \mathrm{MPa}$
\end{tabular}

the base case model using boundary conditions such as wall temperatures and steam inlet conditions.

The initial temperatures and pressures for the base case input model are listed in Table 3.2.1. The initial temperatures of the core radial boundary heat structures were determined by MELCOR using a steady state initialization option and an outer surface temperature of $473 \mathrm{~K}$. These conditions were approximations of the actual experimental conditions at 6000 seconds (actual conditions are found in [5]). The greatest approximation was in initial core component temperatures. A constant temperature of $480 \mathrm{~K}$ was assumed for core components at time 6000 seconds, while Figures 4.1.1-4.1.8 show that the actual temperatures were between $460-510 \mathrm{~K}$. A sensitivity study on these temperatures is described in Section 5.1.3.

The ACRR power history during the DF-4 experiment is shown in Figure 2.2.1. The fission power experienced by the DF-4 test bundle was not measured in the experiment directly, but was inferred using a power coupling relationship derived in [5]. The spatially-dependent power coupling factor was represented by a chopped sinusoidal relation $[5]$

$$
f(x)=1.3+0.4 \sin \left[\pi\left(\frac{x}{\lambda}\right)-0.2\right] \mathrm{W} /(\mathrm{gm} \mathrm{MW}),
$$

where $\lambda$ is the length of the active fuel zone $(50.5 \mathrm{~cm})$ and $x$ is the distance from the bottom of the active fuel, in centimeters. Equation (3.2) was integrated over the axial extent of each cell and multiplied by the cell fuel mass to get the axial peaking factors and the overall bundle power used in the base case model. These normalized peaking factors are compared with Equation (3.2) and the experimental data in Figure 3.2.2. The core axial level peaking factors used in the MELCOR analyses are also given in Table 3.2.2, along with cell elevations. A sensitivity study on total bundle power is discussed in Section 5.5.5.

The inlet steam temperature was set in the MELCOR base case input model using a time-specified inlet volume. The inlet steam temperature used in MELCOR corresponded to the measured inlet steam temperature in Figure 3.2.3. A sensitivity study on the inlet steam temperature is discussed in Section 5.5.2. The mass flow rate of steam into the test bundle is shown in Figure 2.2.2. This data was used to calculate time-specified velocities in flow paths FL101 and FL102, such that $80 \%$ of the flow went through FL101 and $20 \%$ went through FL102. A sensitivity study on the inlet steam flow rate is discussed in Section 5.5.1. 
Table 3.2.2. Starting elevations and power peaking factors for core cells in the MELCOR DF-4 base case input model. Axial levels in the active fuel regions are $5.6 \mathrm{~cm}$ in height.

\begin{tabular}{c|c|c|c|c|c} 
Cell & $\begin{array}{c}\text { Starting } \\
\text { elevation }(\mathrm{cm})\end{array}$ & $\begin{array}{c}\text { Peaking } \\
\text { Factor }\end{array}$ & Cell & $\begin{array}{c}\text { Starting } \\
\text { elevation }(\mathrm{cm})\end{array}$ & $\begin{array}{c}\text { Peaking } \\
\text { Factor }\end{array}$ \\
\hline 101 & -10.8 & 0.00 & 108 & 22.4 & 1.09 \\
102 & -7.6 & 0.00 & 109 & 28.1 & 1.09 \\
103 & -7.5 & 0.00 & 110 & 33.7 & 1.06 \\
104 & 0.0 & 0.83 & 111 & 39.3 & 1.01 \\
105 & 5.6 & 0.92 & 112 & 44.9 & 0.93 \\
106 & 11.2 & 1.00 & 113 & 50.5 & 0.00 \\
107 & 16.8 & 1.06 & 114 & 55.7 & 0.00
\end{tabular}

During the initial phases of the DF-4 experiment, the stainless steel pressure boundary was heated to a temperature sufficient to preclude steam condensation inside the test bundle. The MELCOR input model specified a constant temperature of $473 \mathrm{~K}$ on the outside surfaces of the radial boundary heat structures. During the fuel damage phase of the experiment, these surfaces actually increased in temperature. The temperature used in MELCOR is compared to these time-dependent surface temperatures for several axial levels in Figure 3.2.4. A sensitivity study which varied the surface temperatures on these heat structures is discussed in Section 5.5.4.

The pressure in the test bundle was set to a constant value of $715.7 \mathrm{kPa}$, or about 104 psi, using a time-specified outlet volume. 


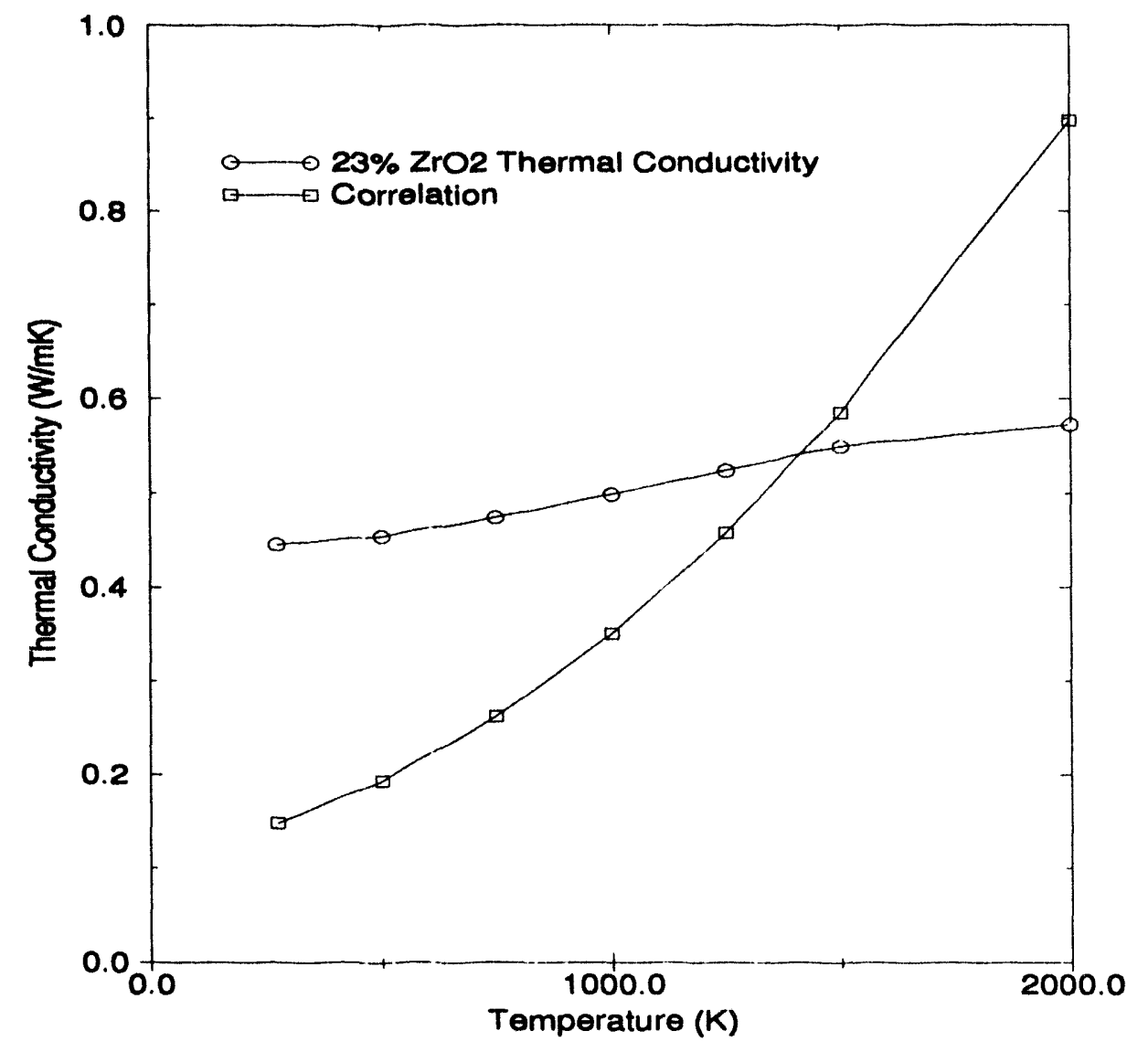

Figure 3.2.1. Empirical thermal conductivity correlation for porous $\mathrm{ZrO}_{2}$ (from [8]) compared to $23 \%$ of MELCOR thermal conductivity for this material. 


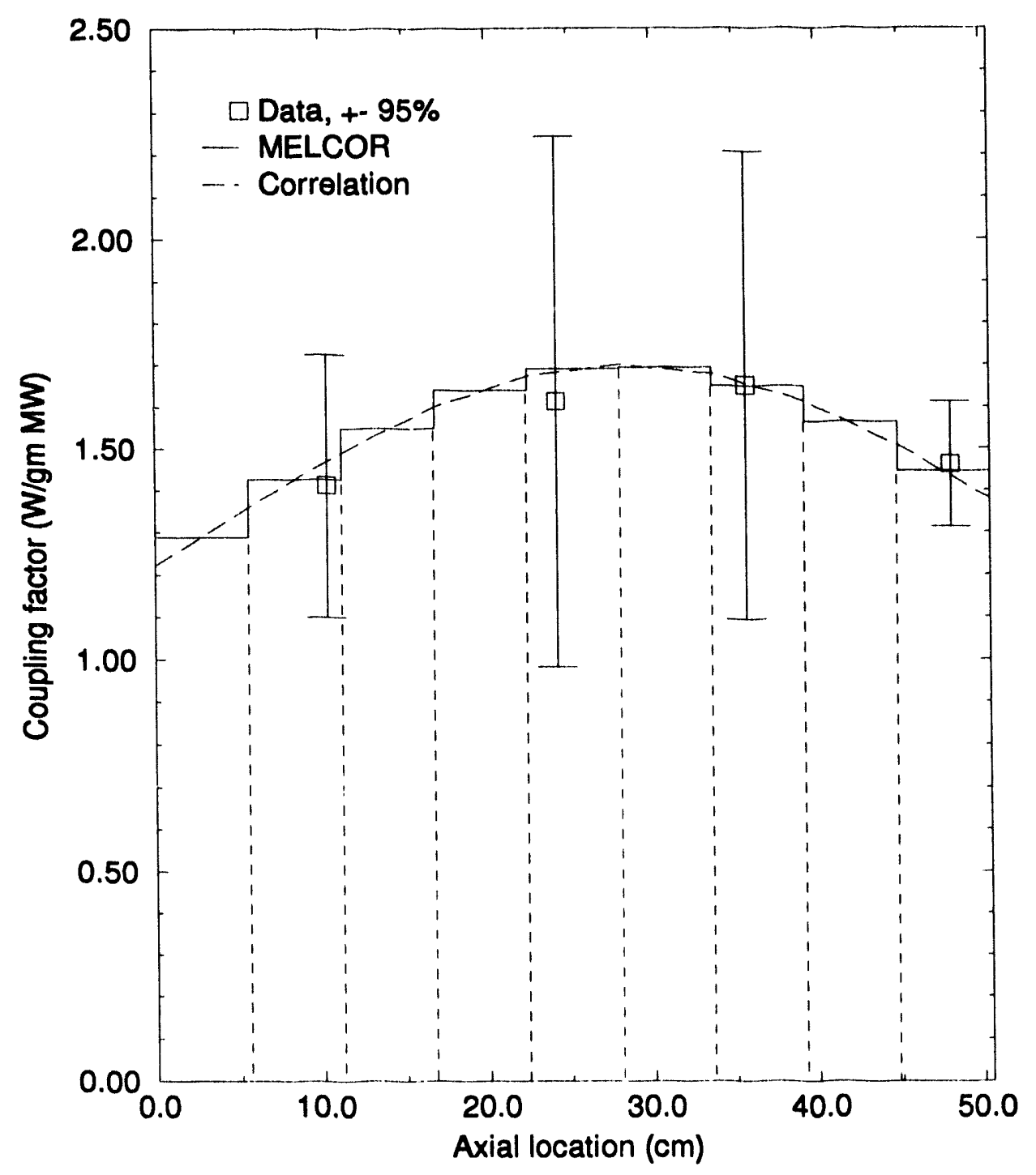

Figure 3.2.2. Cell fission power coupling factors used in MELCOR, compared to data and the correlation derived from those data. Error bars on data values show the $95 \%$ confidence limits. 


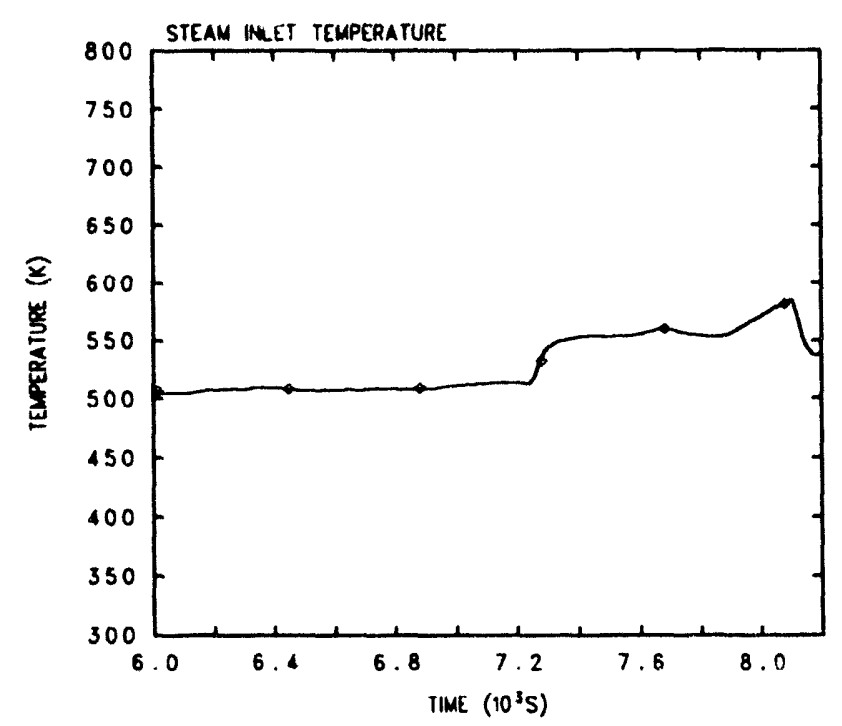

Figure 3.2.3. Steam inlet temperature used in MELCOR.

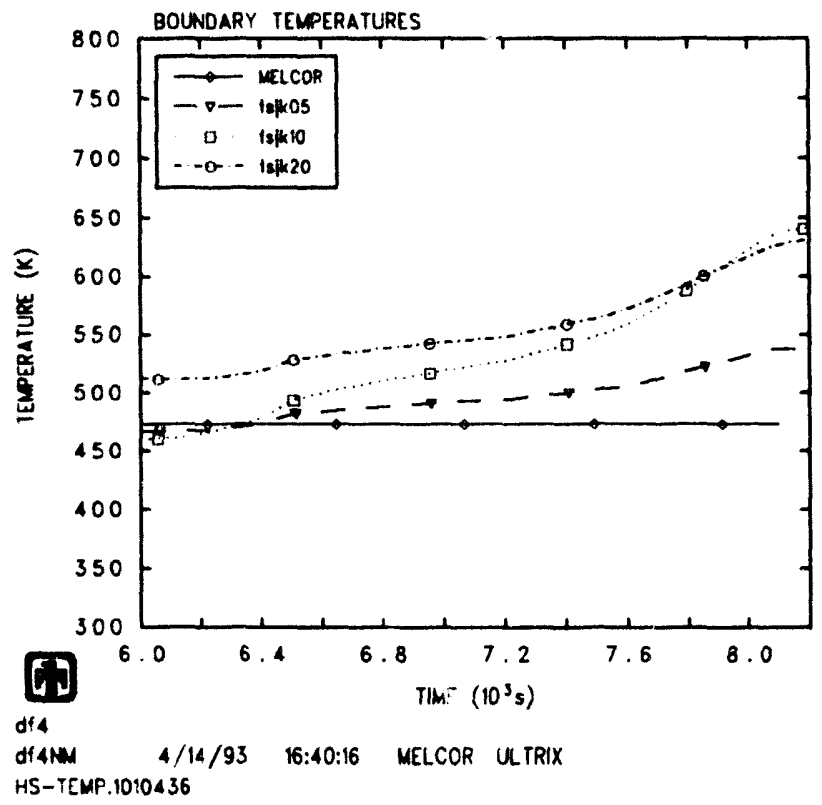

Figure 3.2.4. Constant heat structure outside surface temperature used in MELCOR compared to measured temperatures at several axial levels. TSJK05 is at the top of the bundle and TSJK20 is at the bottom. 


\section{Chapter 4}

\section{MELCOR Base Case Calculation Results}

The results of the MELCOR base case calculation are discussed in this chapter. Note that the base case input model did not use all default MELCOR input options and sensitivity coefficients, but rather used those that either resulted in better agreement with experimental data or that reflected the state of knowledge about either experimental conditions or model accuracy. The base case model was the result of many MELCOR sensitivity studies, some of which are described in the next chapter.

The component temperature results are discussed first, since these quantities were measured directly by the experiment instrumentation. Failure and relocation phenomena are then discussed, and the final core state is analyzed and compared with PIE (Post Irradiation Examination) data. The hydrogen generation behavior in DF-4 is discur 1 last.

Temperature and relocation phenomena are discussed starting at the top of the test bundle and progressing downward, since this was the direction of fuel damage progression in the DF-4 experiment. The control blade behavior is discussed first, because that was the component that began melting first.

\subsection{Temperature Response}

\subsubsection{Control Blade}

The control blade was instrumented with one $\mathrm{Pt} / \mathrm{Rh}$ thermocouple at the $49.5 \mathrm{~cm}$ plane, and two $\mathrm{Pt} / \mathrm{Rh}$ thermocouples at each of the $36.8,25.4$ and $9.6 \mathrm{~cm}$ planes (see Figure 2.1.3).

\section{$49.5 \mathrm{~cm}$ Plane}

The control blade temperature response calculated by MELCOR is compared to the measured temperature at the $49.5 \mathrm{~cm}$ plane in Figure 4.1.1. The general agreement with experimental data was good until about 6900 seconds, when the calculated temperature increased to about $200 \mathrm{~K}$ more than the experimental value. 
The temperature remained high until melting and failure of the control blade at this level.

The control blade reached the eutectic temperature of the $\mathrm{B}_{4} \mathrm{C}$-stainless steel mixture at about 7470 seconds. The materials interactions model in MELCOR held the mixture at a constant temperature while the liquifaction of $\mathrm{B}_{4} \mathrm{C}$ and stainless steel took place. ${ }^{1}$ After all the $\mathrm{B}_{4} \mathrm{C}$ was dissolved, the control blade temperature rose to the stainless steel melting temperature of $1700 \mathrm{~K}$, and the blade was held at this temperature while the stainless steel melted. The control blade at this level was completely consumed after all its stainless steel had melted, at about 7600 seconds. After this point, MELCOR set the temperature of this component to zero.

Other codes modeling the DF-4 experiment also over-predicted temperatures in the upper-most axial level (see Chapter 6). This effect was also observed in the cladding temperature response predicted by MELCOR and other codes (see Figure 4.1.5 and Chapter 6). This would suggest that the power coupling relationship in Equation 3.2 was inaccurate at the upper-most axial level.

\section{$36.8 \mathrm{~cm}$ Plane}

The control blade temperature response at the $36.8 \mathrm{~cm}$ plane calculated by MEL$\mathrm{COR}$ is compared to the measured temperature in Figure 4.1.2. The temperature response for this plane was similar to the response at the $49.5 \mathrm{~cm}$ plane. The control blade temperature at the $36.8 \mathrm{~cm}$ plane rose to the $\mathrm{B}_{4} \mathrm{C}$-stainless steel eutectic reaction temperature, where it leveled off while the $\mathrm{B}_{4} \mathrm{C}$ was consumed by the eutectic reaction. After the depletion of the $\mathrm{B}_{4} \mathrm{C}$, the temperature rose to the stainless steel melting temperature of $1700 \mathrm{~K}$, where it remained while the steel melted. The control blade was calculated to fail shortly thereafter.

The calculated temperature response at this plane was close to the measured temperature response, with the largest difference occurring between 6400 and 7000 seconds. This difference could possibly be attributed to the fact that the MELCOR temperature for the control blade was a bulk temperature, while that measured by the thermocouple was a surface temperature. The calculated temperature differed from the measured temperature by approximately $100 \mathrm{~K}$ between 6500 and 6900 seconds. In other phases, calculated temperatures were within $10 \mathrm{~K}$ of the measured results.

\section{$25.4 \mathrm{~cm}$ Plane}

The control blade temperature response calculated by MELCOR is compared to the measured temperature at the $25.4 \mathrm{~cm}$ plane in Figure 4.1.3. This plot.is similar to that for the $36.8 \mathrm{~cm}$ plane until approximately 7400 seconds. The arrival of molten material from higher axial levels is indicated by the sudden increase in temperature, from $1350 \mathrm{~K}$ to $1500 \mathrm{~K}$, at 7480 seconds. More melt arrived shortly thereafter, bringing the control blade to the eutectic interaction temperature at 7560 seconds. The timing of the overall melt arrival agreed well with experimental data.

\footnotetext{
${ }^{1}$ Note that in the base case input model, the eutectic reaction temperature was set to $1570 \mathrm{~K}$, which was $50 \mathrm{~K}$ larger than the default value (see Sections 3.1 .2 and 5.4.2).
} 


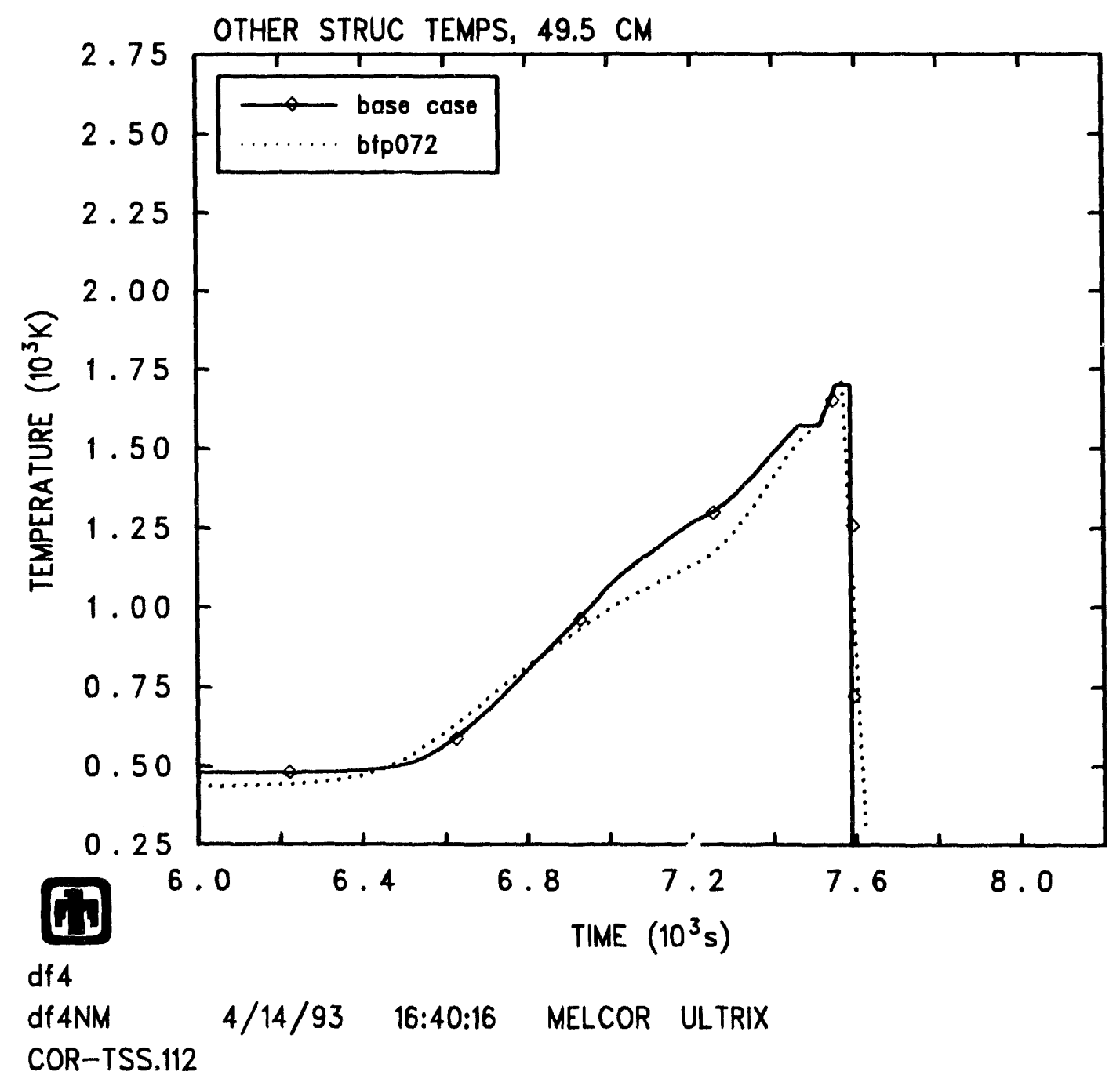

Figure 4.1.1. Measured vs. calculated control blade temperatures at the $49.5 \mathrm{~cm}$ level. 


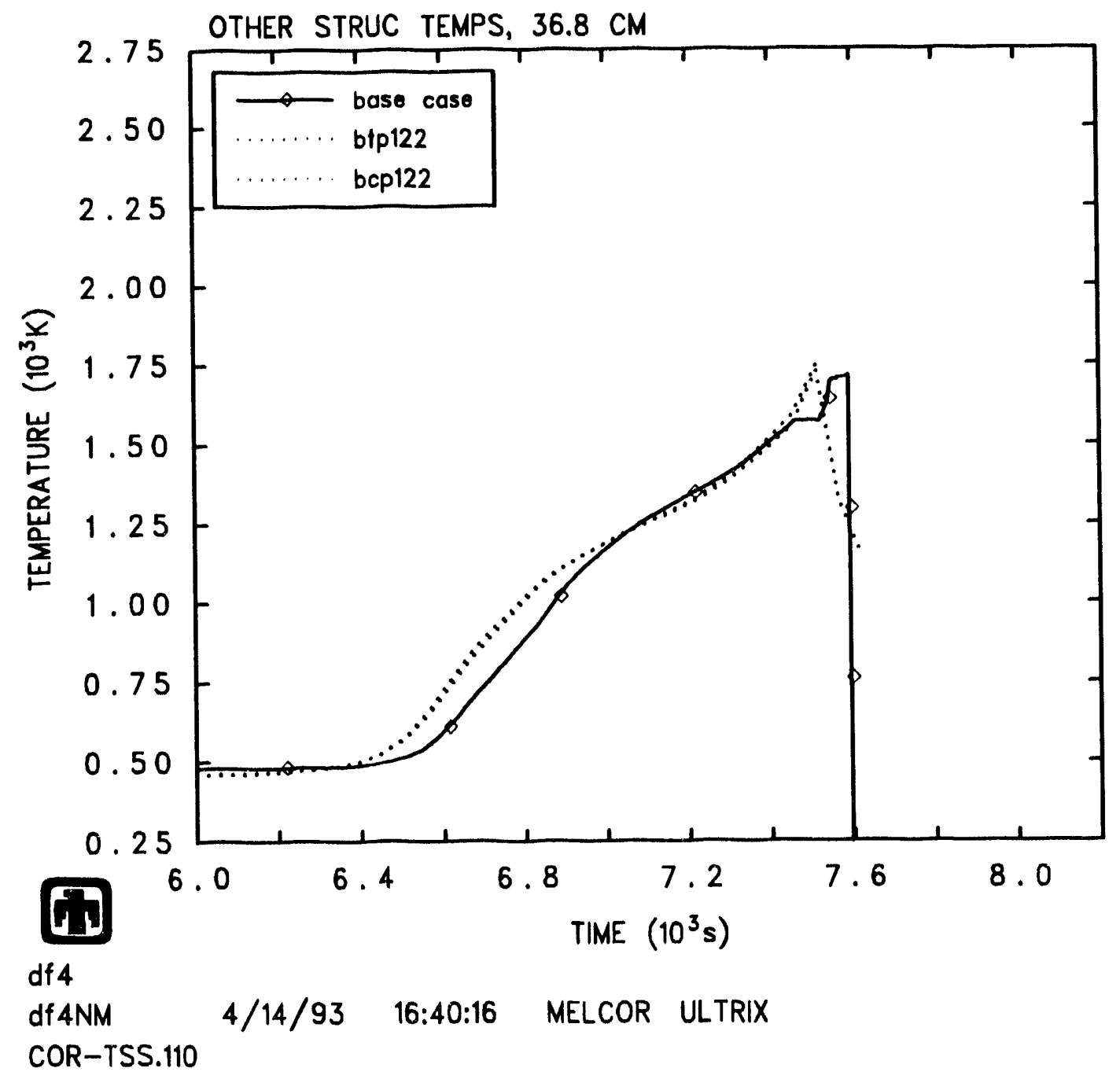

Figure 4.1.2. Measured vs. calculated control blade temperatures at the $36.8 \mathrm{~cm}$ level. 
The eutectic interaction at this level took place much quicker than it did at the $36.8 \mathrm{~cm}$ plane because of the arrival of more relocating melt from higher axial levels. The blade reached the stainless steel melting temperature of $1700 \mathrm{~K}$ at approximately 7650 seconds, and failed shortly afterwards. Although the experimental data showed increasing temperatures beyond the MELCOR-calculated failure time, these data were not reliable because the substrate material for the thermocouple at this location had already melted (as indicated by temperatures in excess of the stainless steel melting temperature). The fact that the MELCOR-calculated temperature of this component arrived at the stainless steel melting temperature at the same time as the measured temperature shows that the failure timing at this level was predicted accurately.

\section{$9.6 \mathrm{~cm}$ Plane}

The control blade temperature response calculated by MELCOR is compared to the measured temperature at the $9.6 \mathrm{~cm}$ plane in Figure 4.1.4. This shows that the temperature at the $9.6 \mathrm{~cm}$ plane was under-predicted by approximately $100 \mathrm{~K}$ before the arrival of melt material at 7000 seconds. The first arrival of molten material was approximately 100 seconds later than measured in the experiment. However, the calculated temperature rose more rapidly to the eutectic interaction temperature, whereas the measured temperature rose to this point in more continuous increments. The calculated versus measured failure times for the control blade at this level (i.e. the time at which the control blade reached the stainless steel melting point) differed by less than 50 seconds. Thus, although the temperature response was different due to discrete relocation events, the overall failure timing was predicted well at this level.

\subsubsection{Cladding}

The cladding was instrumented with one $\mathrm{Pt} / \mathrm{Rh}$ thermocouple at the $49.5 \mathrm{~cm}$ plane, and two $\mathrm{Pt} / \mathrm{Rh}$ thermocouples at each of the $36.8,25.4$ and $9.6 \mathrm{~cm}$ planes (see Figure 2.1.3). In addition, single W/Re thermocouples were used at each axial level to instrument the cladding.

The cladding temperatures measured by $W /$ Re thermocouples are shown with the $\mathrm{Pt} / \mathrm{Rh}$ data in Figures 4.1.5- 4.1.8. These figures show that the temperatures registered by these thermocouples were approximately $300 \mathrm{~K}$ below the temperatures registered by the type $P$ thermocouples, until the latter thermocouples failed at elevated temperatures. This temperature lag was due to the poor conductivity of the $\mathrm{ZrO}_{2}$ sheath that protected the $\mathrm{W} / \mathrm{Re}$ thermocouples from molten materials. Figures 4.1.5- 4.1 .8 also show that the $\mathrm{W} /$ Re thermocouples lagged the $\mathrm{Pt} / \mathrm{Rh}$ thermocouples in time by approximately 25 seconds.

\section{$49.5 \mathrm{~cm}$ Plane}

The cladding temperature response calculated by MELCOR is compared to the measured temperature at the $49.5 \mathrm{~cm}$ plane in Figure 4.1 .5 . The general shape of the 


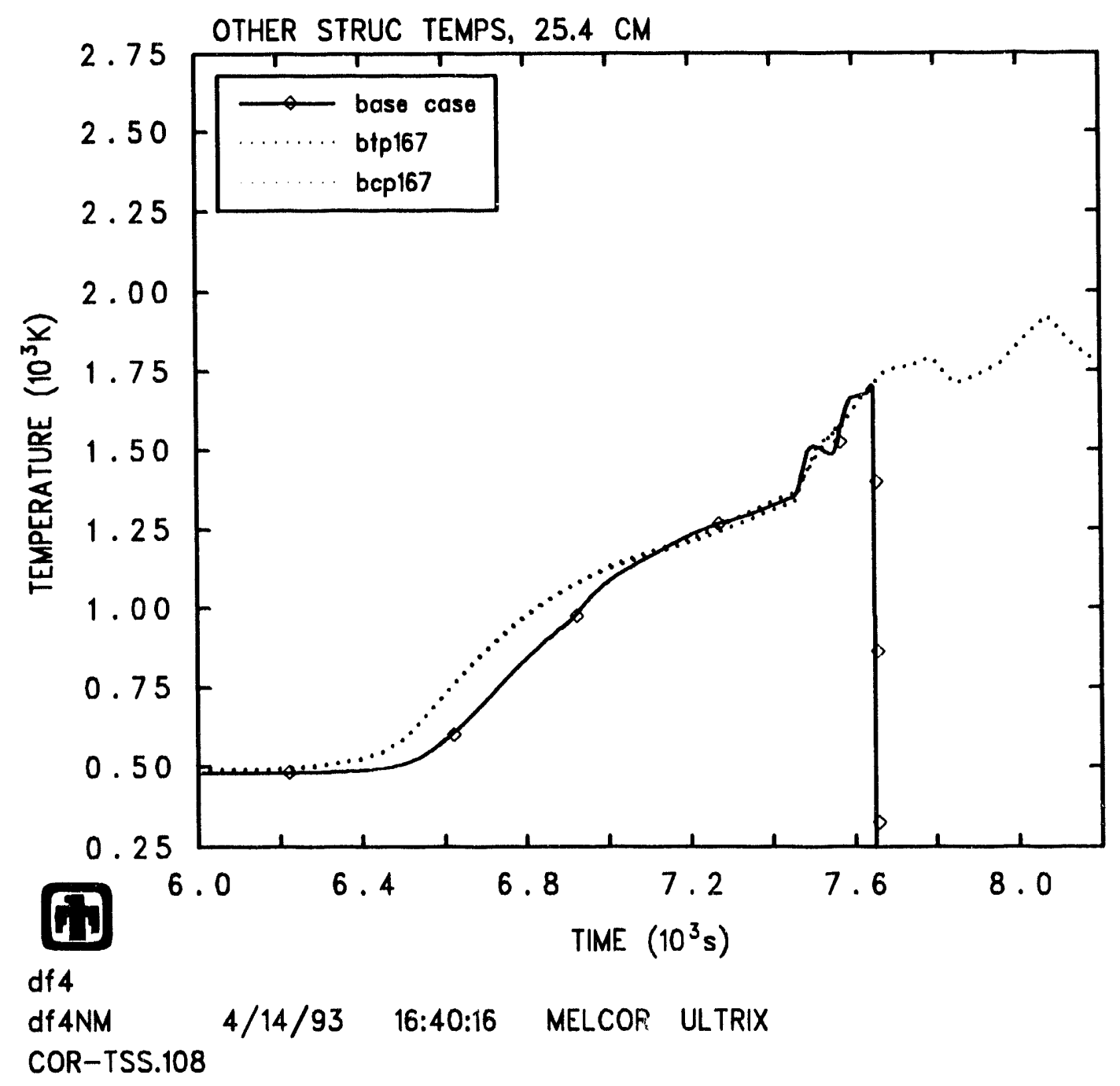

Figure 4.1.3. Measured vs. calculated control blade temperatures at the $25.4 \mathrm{~cm}$ level. 


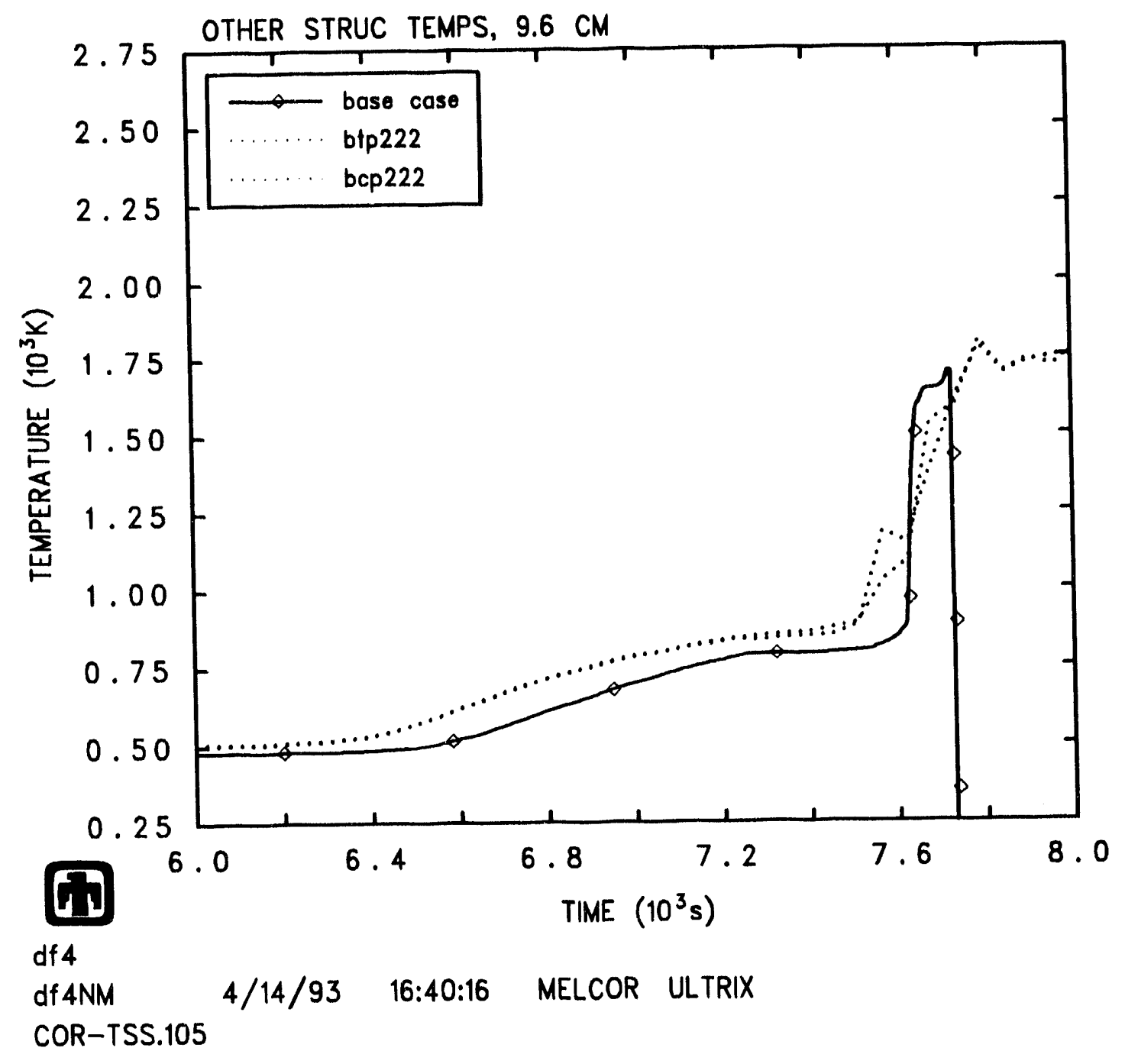

Figure 4.1.4. Measured vs. calculated control blade temperatures at the $9.6 \mathrm{~cm}$ level. 
temperature response was correct; however the MELCOR results show consistently higher temperatures at this plane starting at 6500 seconds. The calculated temperatures diverged from data by about $250 \mathrm{~K}$ by 7200 seconds, then drew closer until the beginning of rapid oxidation at 7550 seconds. A similar effect was observed in the control blade temperature response, and could be attributed to the inaccuracy of the power coupling factor used to calculate bundle peaking factors (this conclusion is reinforced by the results of other codes' DF-4 analyses, which showed the same behavior - see Section 6.1 and Figure 6.1.5). Rapid oxidation at this level began when the clad temperature reached a temperature of $1750 \mathrm{~K}$. This occurred at the $49.5 \mathrm{~cm}$ plane at 7550 seconds. The clad at this level quickly reached the zircaloy melting temperature, and was completely molten or oxidized by 7625 seconds.

\section{$36.8 \mathrm{~cm}$ Plane}

The cladding temperature response calculated by MELCOR is compared to the measured temperature at the $36.8 \mathrm{~cm}$ plane in Figure 4.1.6. The temperature response at this level closely matched the measured temperature response for all but the final period of oxidation. At this point, the temperatures diverged by approximately $50 \mathrm{~K}$. Autocatalytic oxidation was calculated to begin at the measured time of rapid oxidation. The clad at this level remained at a temperature close to the zircaloy melting temperature until about 7650 seconds, at which time the cladding component failed.

\section{$25.4 \mathrm{~cm}$ Plane}

The cladding temperature response calculated by MELCOR is compared to the measured temperature at the $25.4 \mathrm{~cm}$ plane in Figure 4.1.7. The temperature response at this level matched the measured temperature response for most of the time, diverging by approximately $100 \mathrm{~K}$ just before the start of rapid oxidation. Figure 4.1 .7 shows that the calculated start of rapid oxidation occurred at about the measurcd oxidation time of 7610 seconds. The clad at this level remained close to the melting temperature of zircaloy until failure at 7700 seconds.

\section{$9.6 \mathrm{~cm}$ Plane}

The cladding temperature response calculated by MELCOR is compared to the measured temperature at the $9.6 \mathrm{~cm}$ plane in Figure 4.1.8. The calculated temperature at this plane was approximately $50 \mathrm{~K}$ lower than that measured. This was consistent with the temperature results for the control blade, which were also lower than measured (see Figure 4.1.4). The arrival of debris at this level is indicated by the sudden increases in temperature after 7600 seconds, which was very close to the measured debris arrival time. This was consistent with the results for the canister (see Figure 4.1.10). 


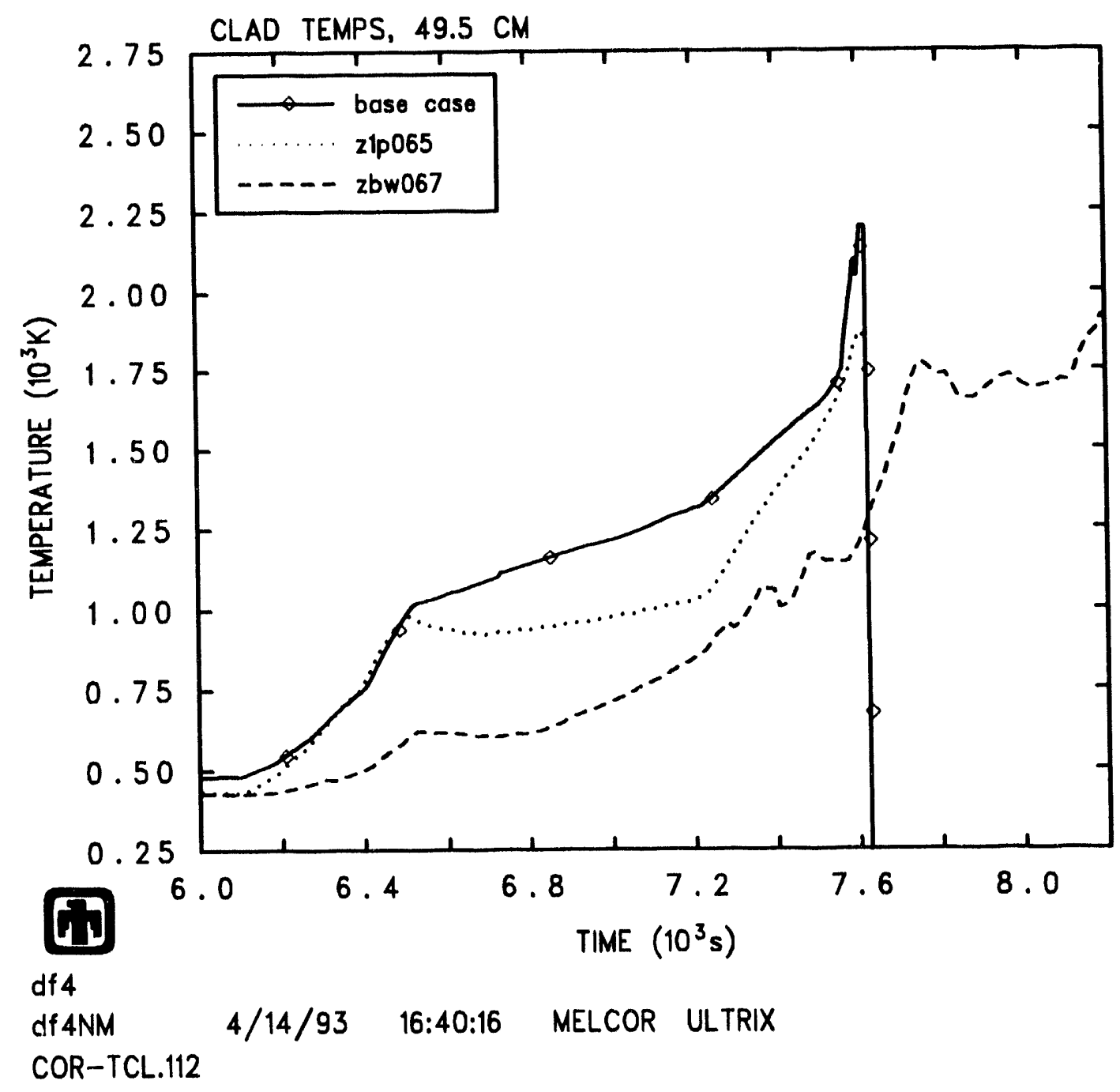

Figure 4.1.5. Measured vs. calculated fuel rod cladding temperatures at the 49.5 $\mathrm{cm}$ level. 


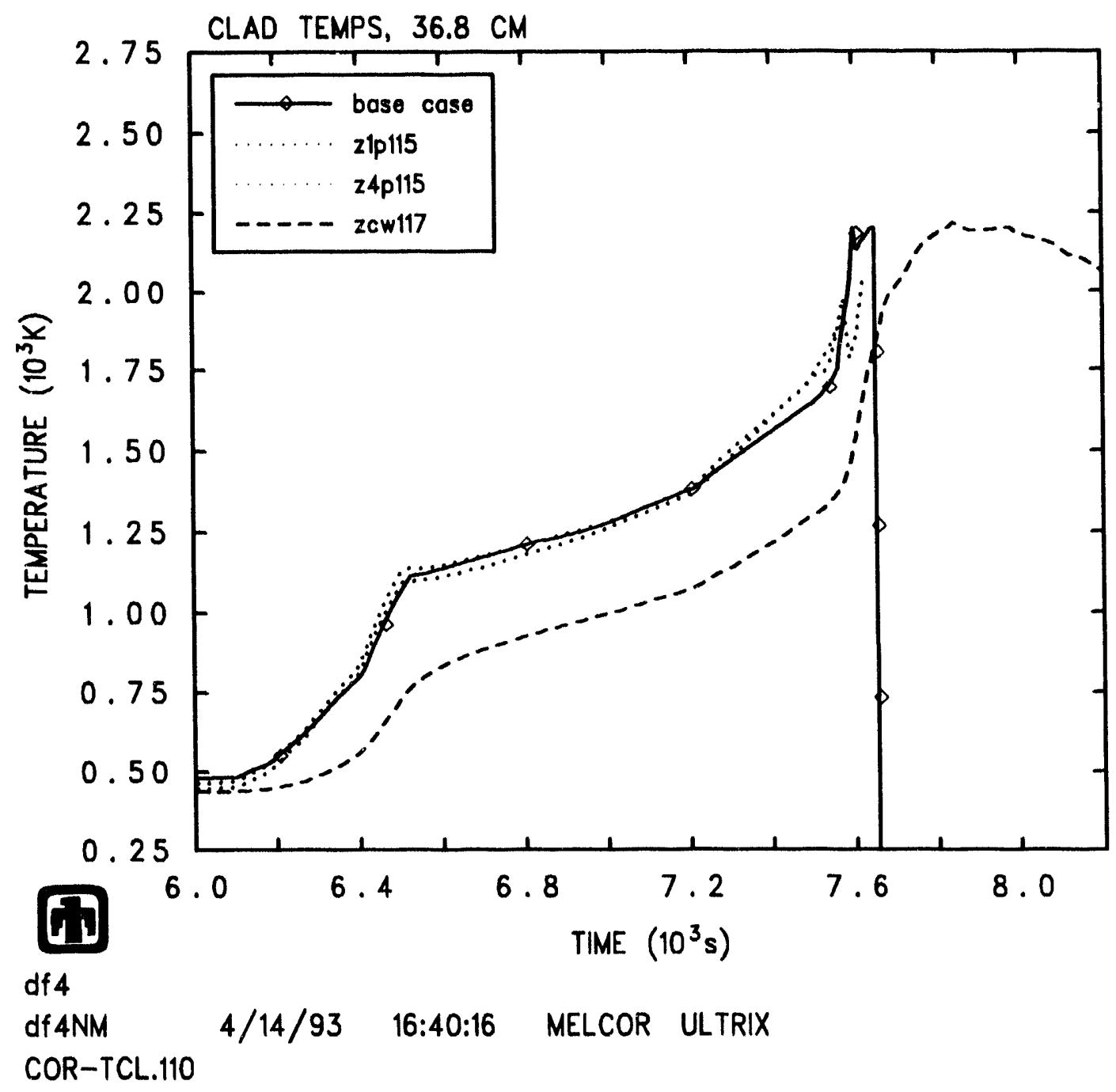

Figure 4.1.6. Measured vs. calculated fuel rod cladding temperatures at the 36.8 cm level. 


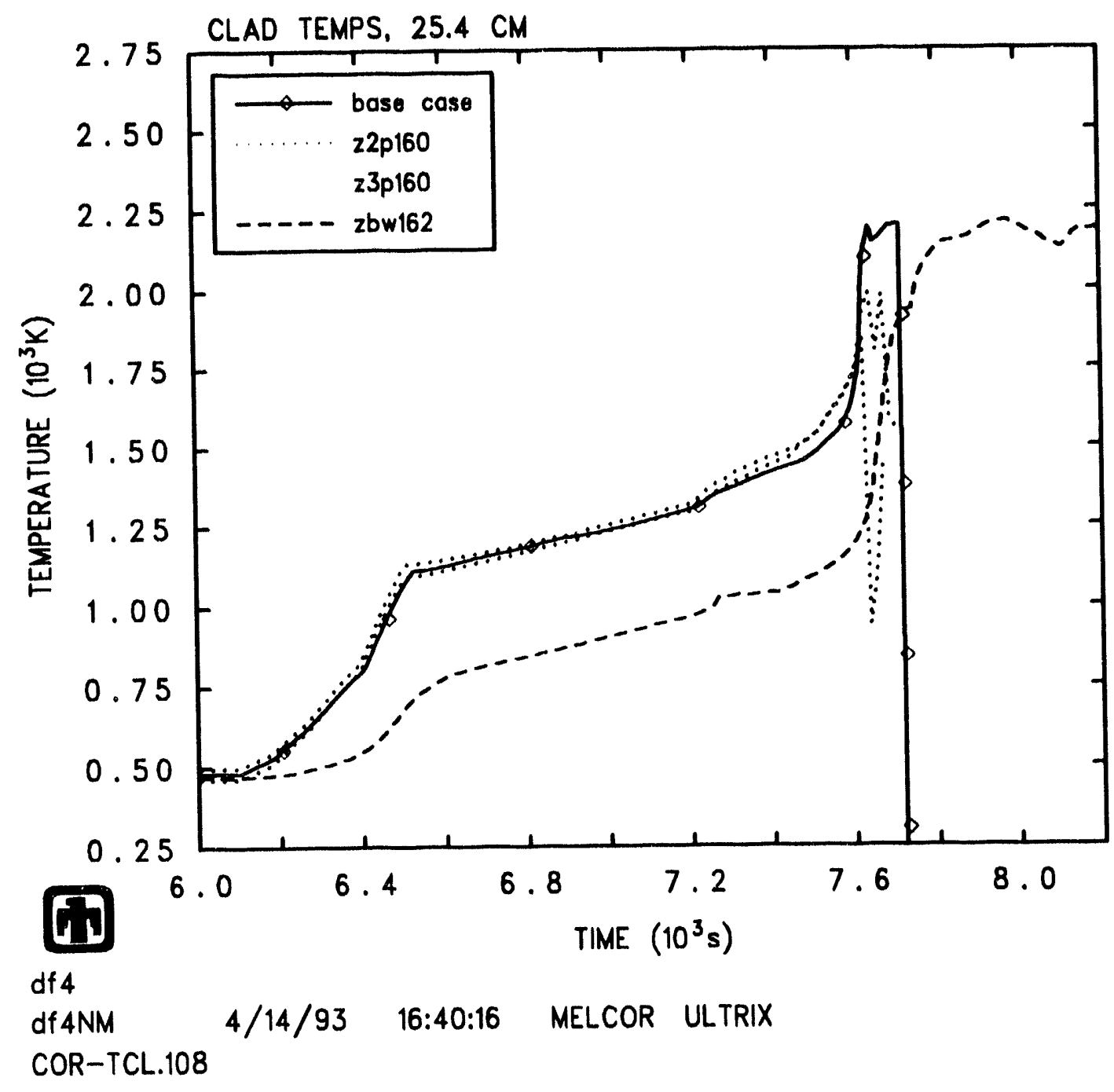

Figure 4.1.7. Measured vs. calculated fuel rod cladding temperatures at the 25.4 cm level. 


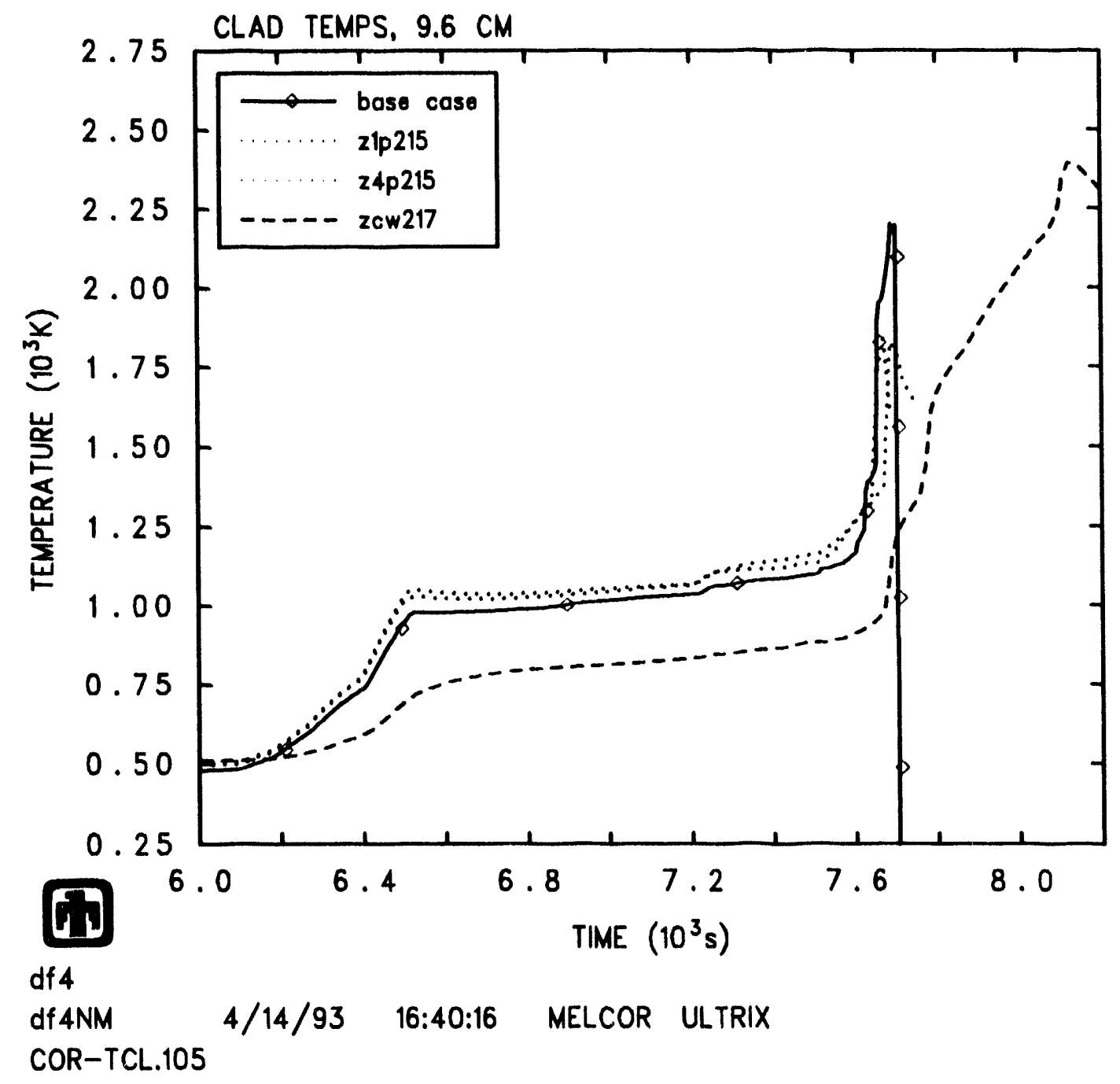

Figure 4.1.8. Measured vs. calculated fuel rod cladding temperatures at the 9.6 cm level. 


\subsubsection{Canister}

The canister in the DF-4 experiment was instrumented with one W/Re thermocouple at the $49.5 \mathrm{~cm}$ and $9.6 \mathrm{~cm}$ planes, and two W/Re thermocouples at each of the 36.8 and $25.4 \mathrm{~cm}$ planes (see Figure 2.1.3). These types of thermocouples showed temperature and time lags of $300 \mathrm{~K}$ and 25 seconds, respectively, in the canister temperatures.

\section{5 and $36.8 \mathrm{~cm}$ Planes}

The calculated temperature response of the canister-b component ${ }^{2}$ at the 49.5 and $36.8 \mathrm{~cm}$ planes is shown in Figure 4.1.9. The response of the W/Re thermocouples at these planes is also shown. The calculated temperatures for the $49.5 \mathrm{~cm}$ plane were more than $500 \mathrm{~K}$ greater than the measured temperatures, after applying the $300 \mathrm{~K}$ correction for the type $\mathrm{C}$ thermocouples. This was the same behavior shown by the cladding and control blade temperatures at this plane. The calculated onset of rapid oxidation, indicated by a sudden increase in the heatup rate, was within the 25 second time lag correction of the measured onset of rapid oxidation shown for the $49.5 \mathrm{~cm}$ plane in Figure 4.1.9.

The calculated temperature behavior at the $36.8 \mathrm{~cm}$ plane shown in Figure 4.1.9 was close to the corrected measured temperatures, and the onset of rapid oxidation was also predicted accurately.

Both the 36.8 and $49.5 \mathrm{~cm}$ planes showed a decrease in temperature approximately 75 seconds after rapid oxidation began. This was due to the relocation of the fuel rod material from these levels, which removed the heating source for the canister component. The canister components at these planes then fell to the temperature of the steam flowing at those levels. The measured temperatures did not show this effect, possibly because of the temperature and time lags of these thermocouples.

\section{4 and $9.6 \mathrm{~cm}$ Planes}

The temperature response of the canister-b component in the 25.4 and $9.6 \mathrm{~cm}$ planes is shown in Figure 4.1.10. The response of the W/Re thermocouples at these planes is also shown. The temperature response and onset of rapid oxidation at the $25.4 \mathrm{~cm}$ plane corresponded to the measured canister temperature and oxidation timing at that plane, after correcting for the time and temperature lags. The calculated temperatures in the later parts of the oxidation subphase were lower than those measured. This was due to the fact that MELCOR does not allow fuel material in the particulate debris component to generate fission power (note that this is only a problem for in-pile experiments and ATWS-type accident scenarios, where core melt progression is driven by fission power rather than decay heat). Since there was still significant reactor power being applied after fuel began relocating, there was probably a significant amount of fission power generated in the fuel debris. The calculated

\footnotetext{
${ }^{2}$ Note that in the MELCOR base case input model all canister mass was allocated to the canister-b component, or that part of the canister that is adjacent to the control blade.
} 


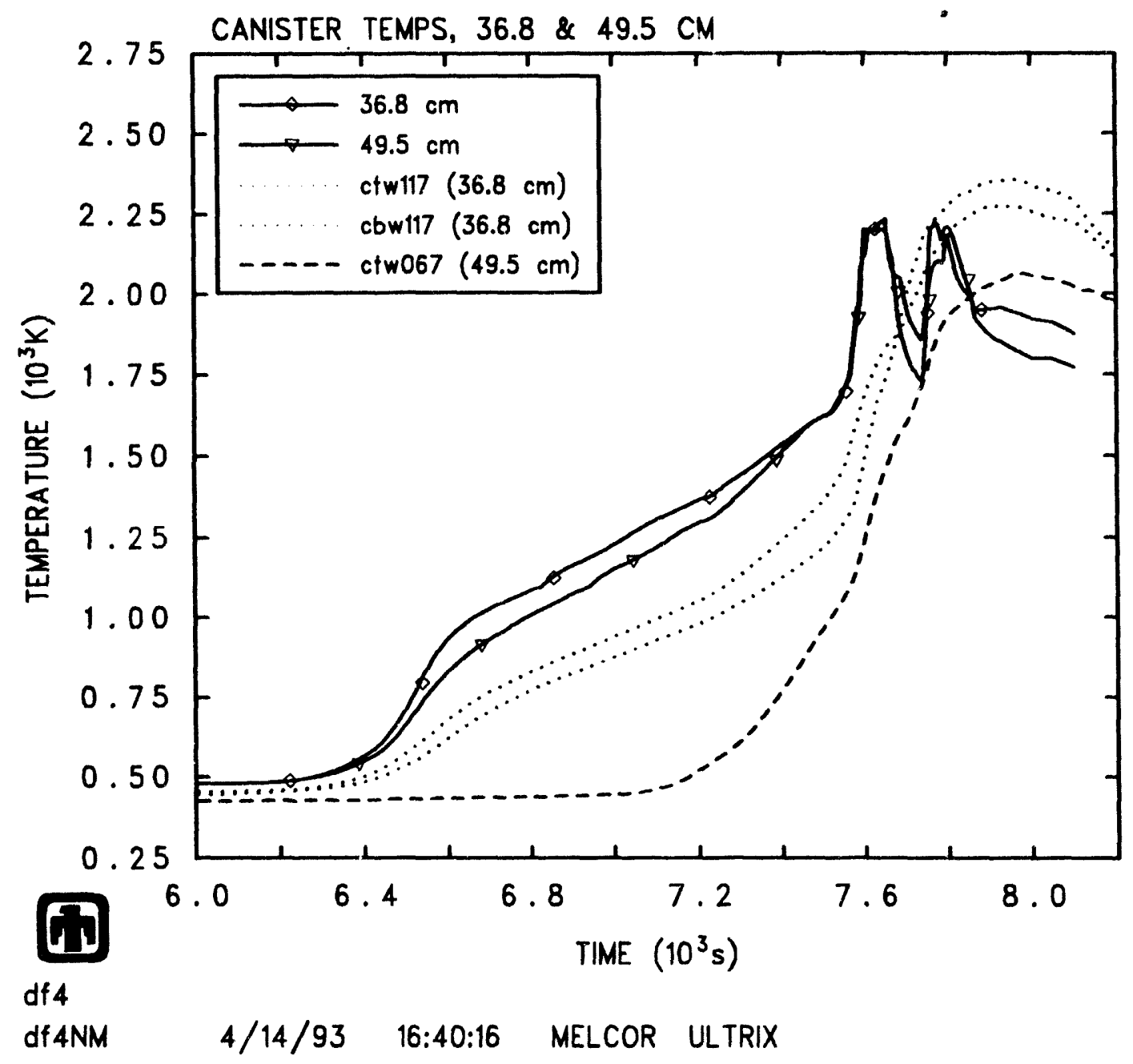

Figure 4.1.9. Measured vs. calculated canister temperatures at the 49.5 and 36.8 cm levels. 
temperatures in the $25.4 \mathrm{~cm}$ plane did not show the decrease in temperature experienced in higher planes. The fuel material at this plane did not relocate downward, as the fuel at higher planes did.

The calculated temperaiure hehavior at the $9.6 \mathrm{~cm}$ plane shows the arrival of relocating melt about 100 seconds early, the same time difference shown in the cladding and control blade temperatures in Figures 4.1.8 and 4.1.4, respectively. The temperatures at this plane in the later part of the oxidation subphase were higher than those at the $25.4 \mathrm{~cm}$ plane, due to the higher amounts of debris at this level.

\subsubsection{Component Temperature Response Overview}

The temperatures across the test bundle in the cladding and control blade components are shown in Figures 4.1.11 and 4.1.12, respectively. In the cladding component, the experimental data indicated that the upper two levels $(36.8$ and $49.5 \mathrm{~cm}$ ) began oxidizing at about the same time. This is consistent with MELCOR calculations which showed that oxidation started at cell 111, which was centered at $42.5 \mathrm{~cm}$. The oxidation front propagated down to the $25.4 \mathrm{~cm}$ plane in approximately $75 \mathrm{sec}$ onds, and to the $9.6 \mathrm{~cm}$ plane in approximately 175 seconds. The steady increase in temperature at the $25.4 \mathrm{~cm}$ plane indicates that oxidation began as a result of the propagation of the oxidation front down the fuel rod, while the large increase in temperature at the $9.6 \mathrm{~cm}$ plane was probably the result of debris relocating to that level.

In Figure 4.1.12, the thermal signature left by the melt candling down the control blade can be seen. The material in the upper two planes, at 36.8 and $49.5 \mathrm{~cm}$, started melting at about the same time. Shortly afterwards, melt arrived at the $25.4 \mathrm{~cm}$ plane. 'This plane was not heated to the eutectic interaction temperature of $1570 \mathrm{~K}$ until the stainless steel started melting at the 36.8 and $49.5 \mathrm{~cm}$ planes and this melt arrived at the $25.4 \mathrm{~cm}$ plane (shown by the second steep increase in control blade temperature). Slow candling was shown by the relatively late temperature increase at the $9.6 \mathrm{~cm}$ plane.

The temperatures of the fuel, cladding, canister-b and other structure components at the $36.8 \mathrm{~cm}$ plane are shown together in Figure 4.1.13. This figure indicates that at elevated temperatures, all components were very close in temperature. This was the result of the test bundle equilibration sub-phase of the experiment (between 6500 and 7200 seconds), where the bundle power was held constant to allow all components to equilibrate in temperature. Rapid oxidation began in the cladding component, but quickly propagated to the canister-b component. The other structure component also showed a temperature rise at about the same time, which brought the blade to the stainless steel melting temperature, resulting in total melting and failure of the control blade. Note that the eutectic interaction in the control blade started before oxidation, as indicated by the flat spot in the control blade temperatures in Figure 4.1 .13 before the steep increase in cladding and canister temperatures.

Component temperatures at the $25.4 \mathrm{~cm}$ plane, shown in Figure 4.1.14, behaved in a similar manner as those at $36.8 \mathrm{~cm}$. The one exception is the increase in other structure temperature caused by the candling of molten eutectic down the control 


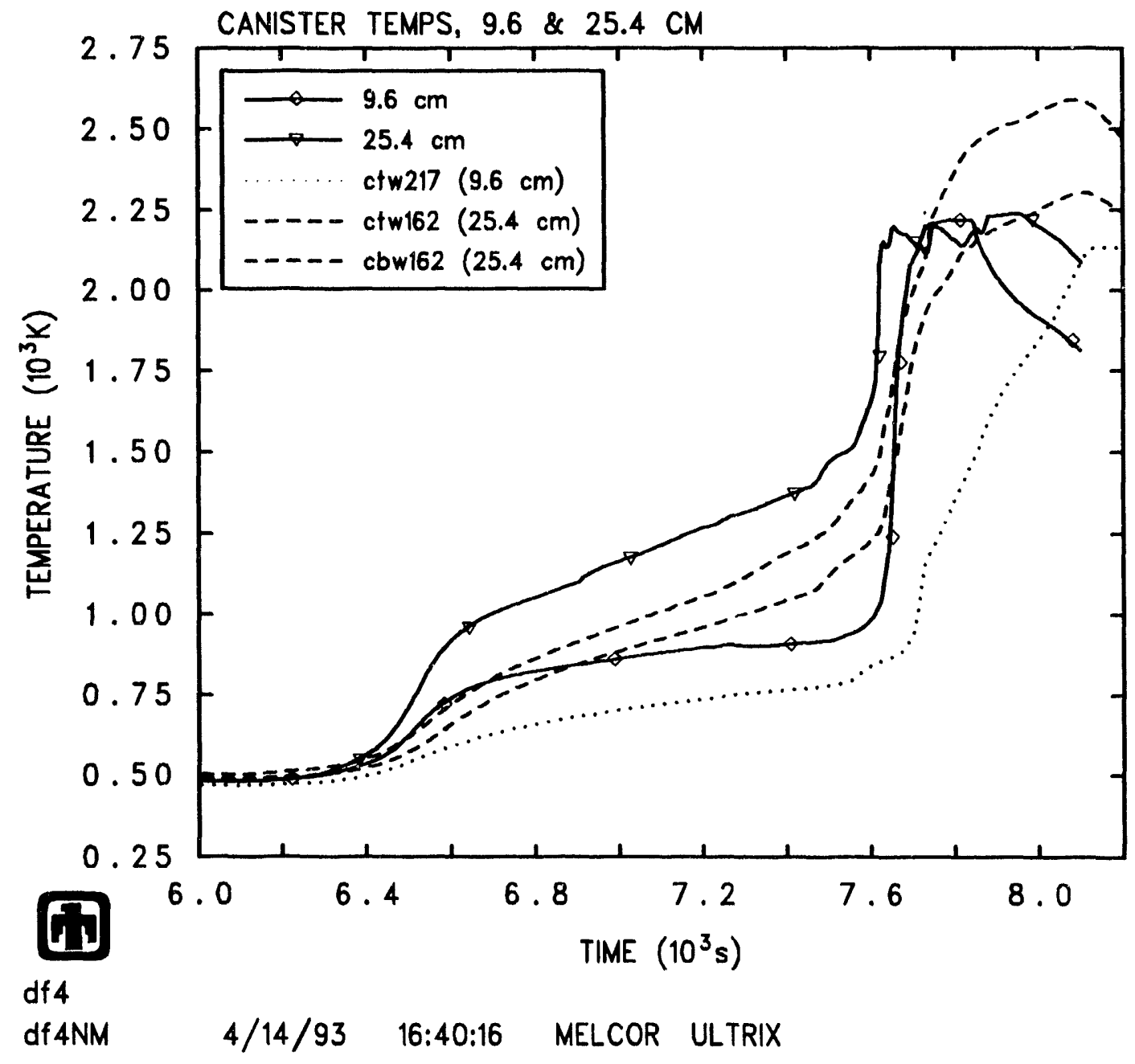

Figure 4.1.10. Measured vs. calculated canister temperatures at the 25.4 and 9.6 cm levels. 
blade. This caused the control blade temperature to exceed the cladding and canister temperature by a small amount, until the arrival of the oxidation front.

\subsection{Melting and Relocation}

The discussion of the temperature response in the last section gives some indication of the melting and relocation of bundle materials. In this section a more detailed discussion of melting and relocation is given. The initial state of the fuel bundle is given first, in the format that is used to discuss core degradation and relocation. The damage progression is then discussed, starting at the beginning of the oxidation at 7500 seconds and proceeding to the later stages of bundle damage.

\subsubsection{Initial State}

The initial material distribution in the test bundle is shown in Figure 4.2.1. Shown are volume fraction plots, where the shaded areas represent the fraction of total volume in each core cell occupied by the designated components and materials at each level. Each major component is represented in a different frame, with the fuel and cladding components on the upper left of the figure, the particulate debris on the upper right, the canister-b component on the lower left, and the other structure component on the lower right. Each plot shows the locations of intact and conglomerate debris materials for the designated component, with the shading used for e ch material shown at the right of the figure.

The $\mathrm{UO}_{2}$ in the uppermost and lower-most axial cells in Figure 4.2 .1 represents spacer material (the actual material used in the spacers was $\mathrm{Al}_{2} \mathrm{O}_{3}$ ), and was not allocated fission power in the base case MELCOR model. Note that the core plate was represented by stainless steel in the other structure component, and that there were no core components in the lower plenum cell. In the following subsections, levels are referred to by their starting elevations. For reference, the starting elevations of all axial levels are given in Table 3.2.2. Each axial level in the active fuel region was $5.6 \mathrm{~cm}$ in height.

\subsubsection{Early Oxidation Subphase (7450-7625 seconds)}

The start of significant test bundle damage in the DF-4 experiment was at approximately 7450 seconds. The core material states between 7450 and 7625 seconds, in 50 second increments, are shown in Figures 4.2.2 $\cdots 4.2 .6$.

At 7450 seconds, Figure 4.2 .2 shows the early stages of cladding and canister oxidation between 25 and $50 \mathrm{~cm}$. At 7500 seconds, $\mathrm{B}_{4} \mathrm{C}$ (CRP in Figure 4.2.3) had started relocating from axial level $11(39.3 \mathrm{~cm}$ elevation). Note that the measured temperatures at the 36.8 and $49.5 \mathrm{~cm}$ planes in Figures 4.1 .1 and 4.1 .2 were below the melting point of stainless steel $(1700 \mathrm{~K})$ at 7500 seconds. This shows the beginning of the eutectic interaction between the $\mathrm{B}_{4} \mathrm{C}$ and stainless steel. The eutectic interaction products became molten and candled down approximately $10 \mathrm{~cm}$, to axial levels 8 and 


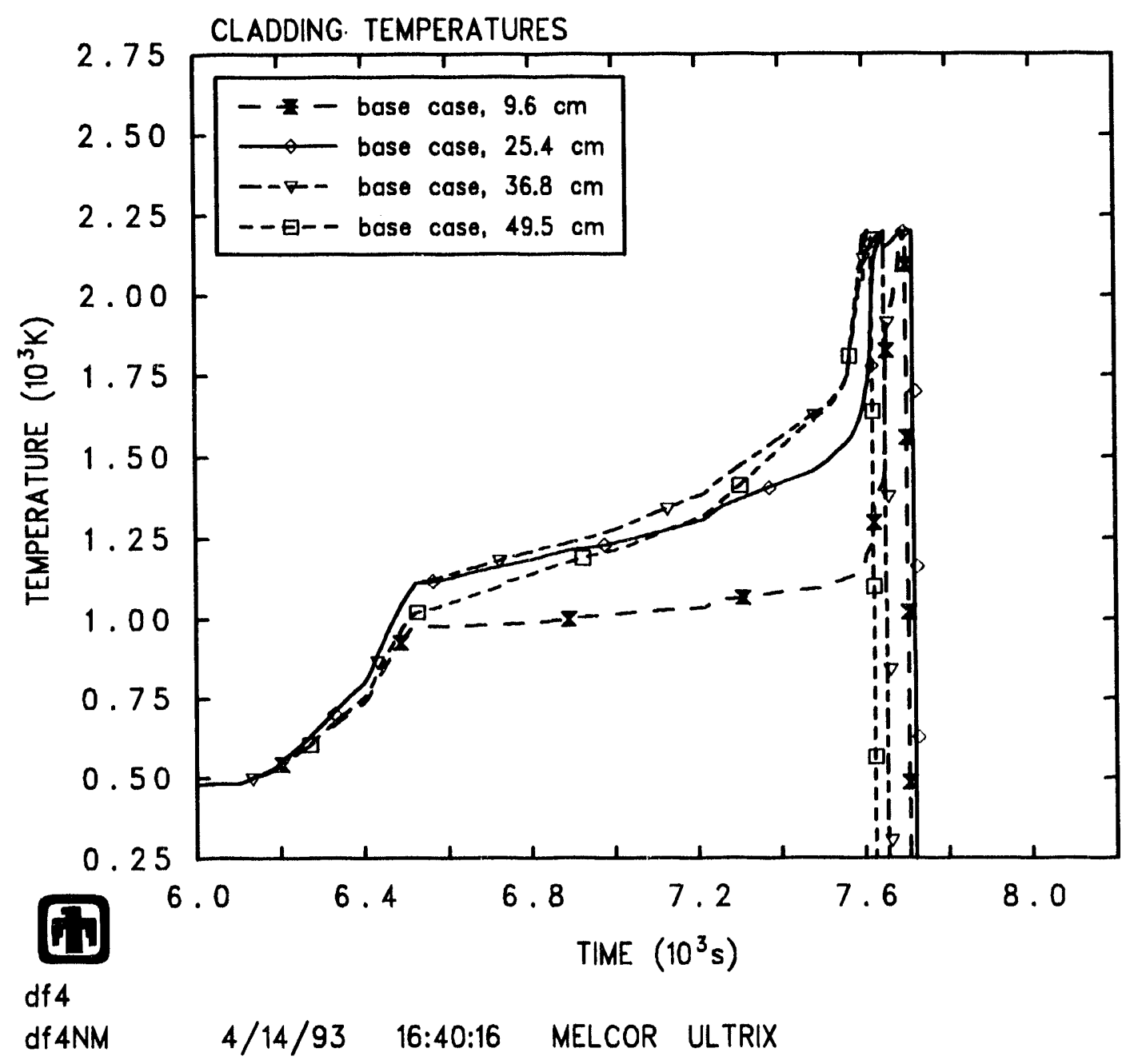

Figure 4.1.11. Cladding temperatures at the four measured axial planes, for the base case input model. 


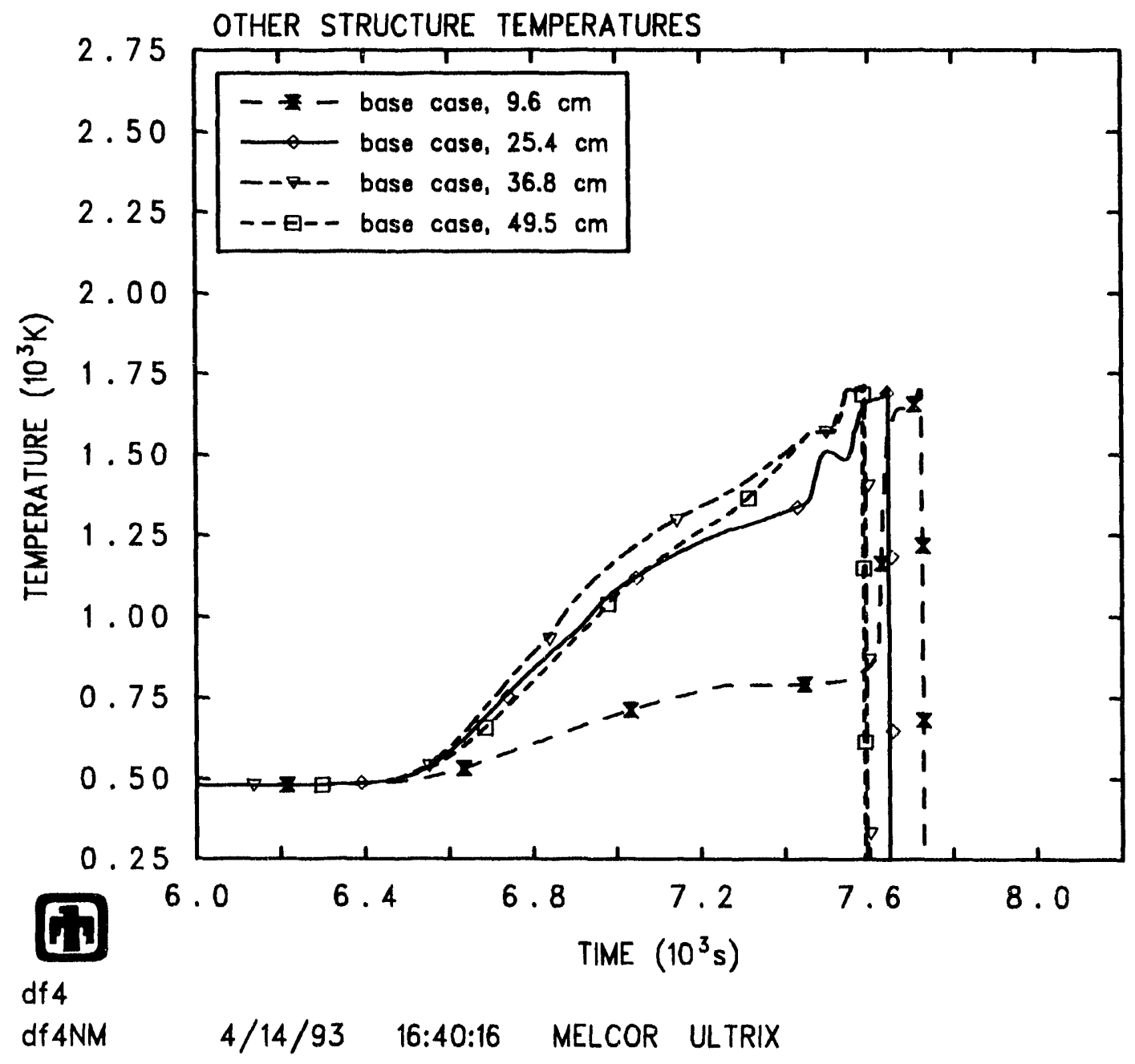

Figure 4.1.12. Control blade temperatures at the four measured axial planes, for the base case input model. 


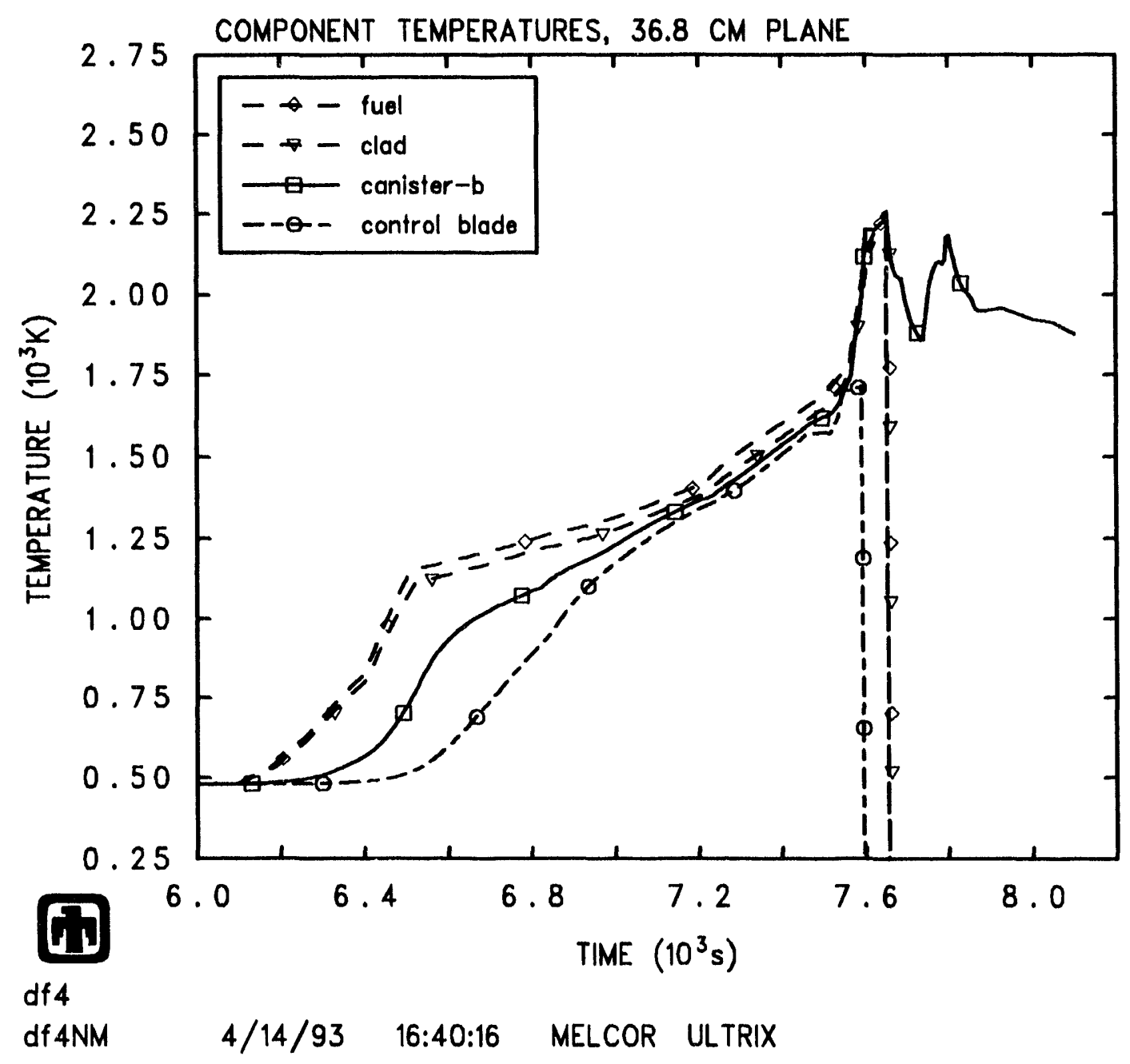

Figure 4.1.13. Component temperatures for the fuel, cladding, canister-b and other structure (control blade) components, at the $36.8 \mathrm{~cm}$ plane, for the base case input model. 


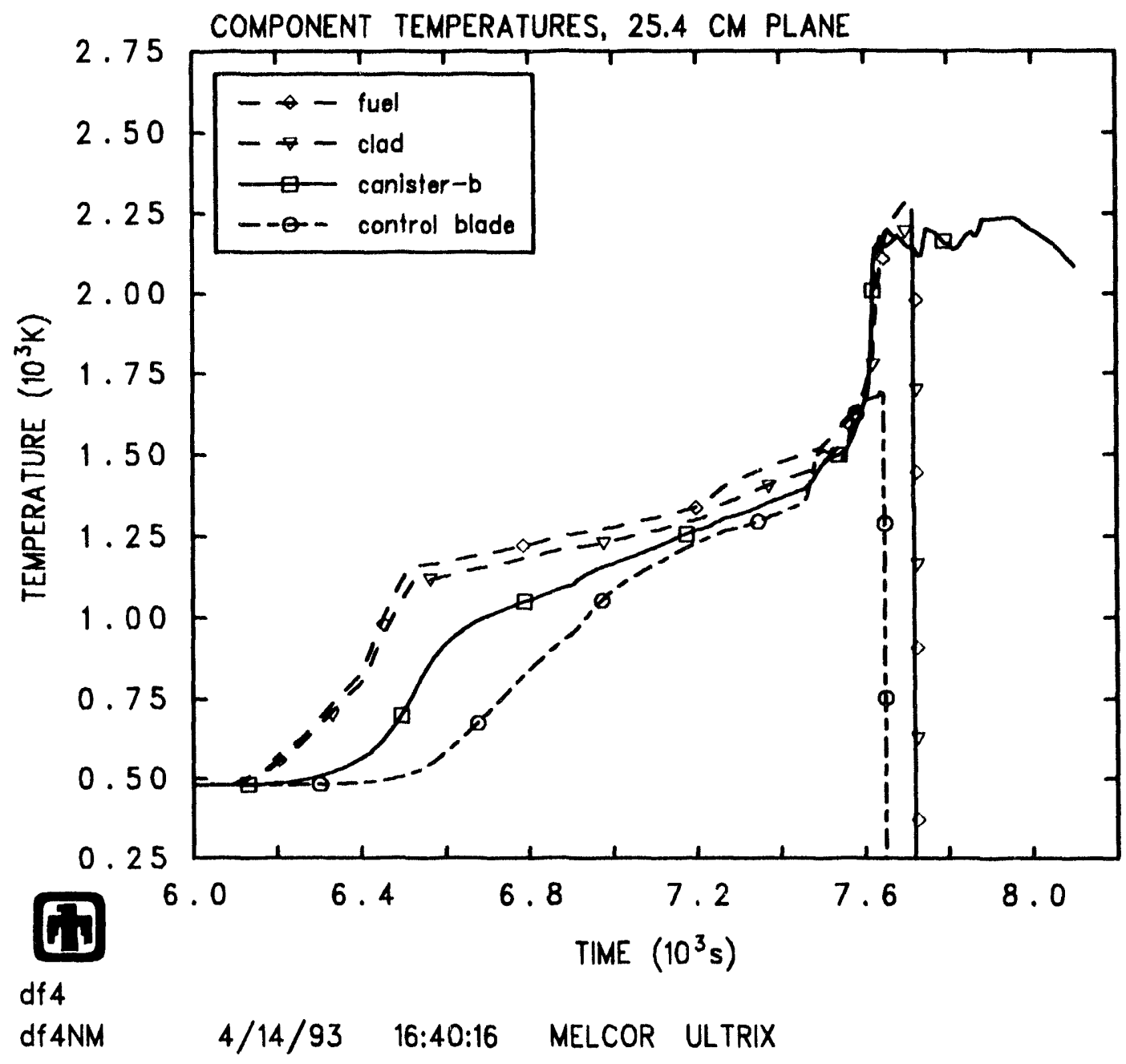

Figure 4.1.14. Component temperatures for the fuel, cladding, canister-b and other structure (control blade) components, at the $25.4 \mathrm{~cm}$ plane, for the base case input model. 

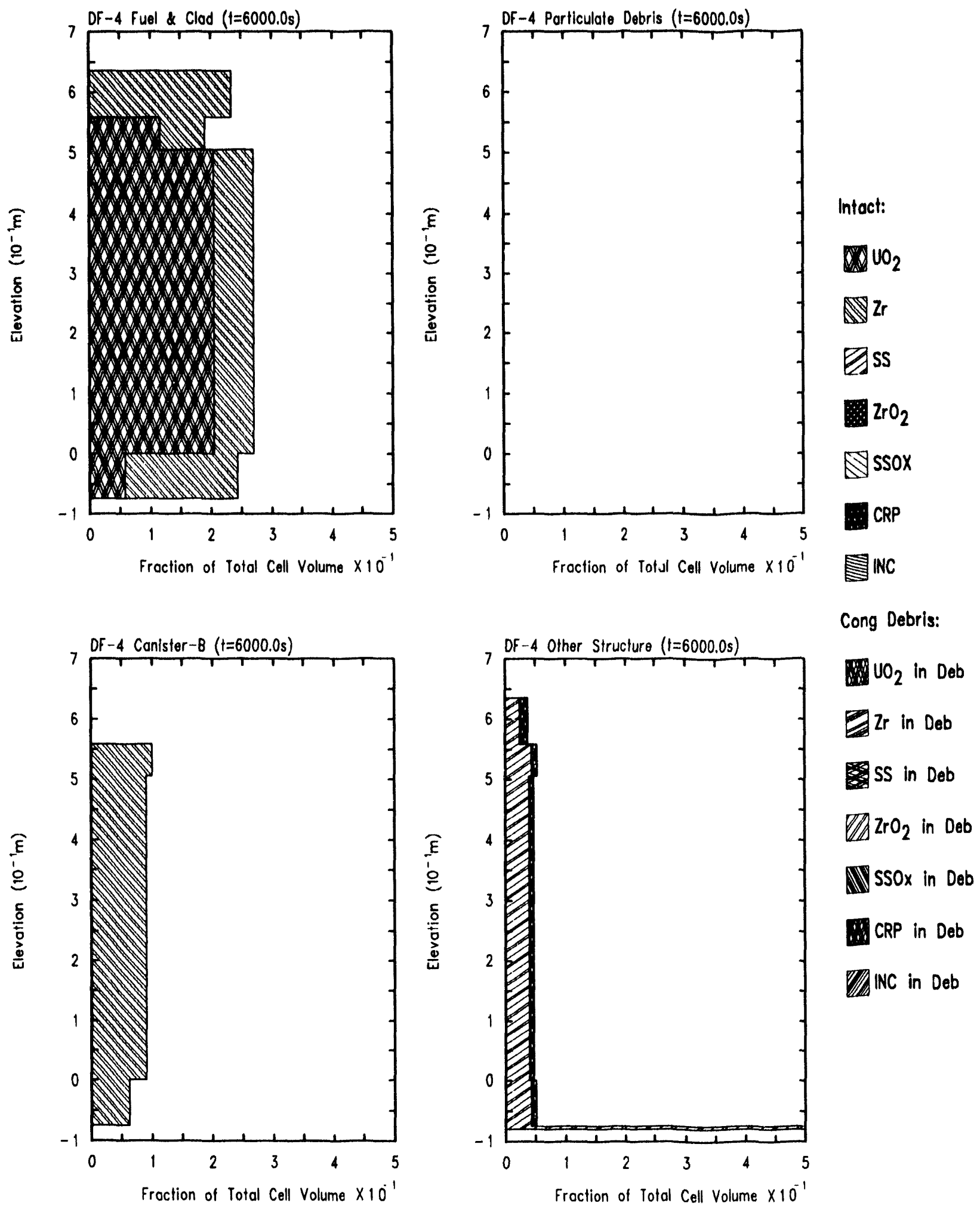

Figure 4.2.1. Initial locations (at 6000 seconds) of fuel and clad (upper left), particulate debris (upper right), canister-b (lower left) and other structure (lower right) component materials in DF-4. 

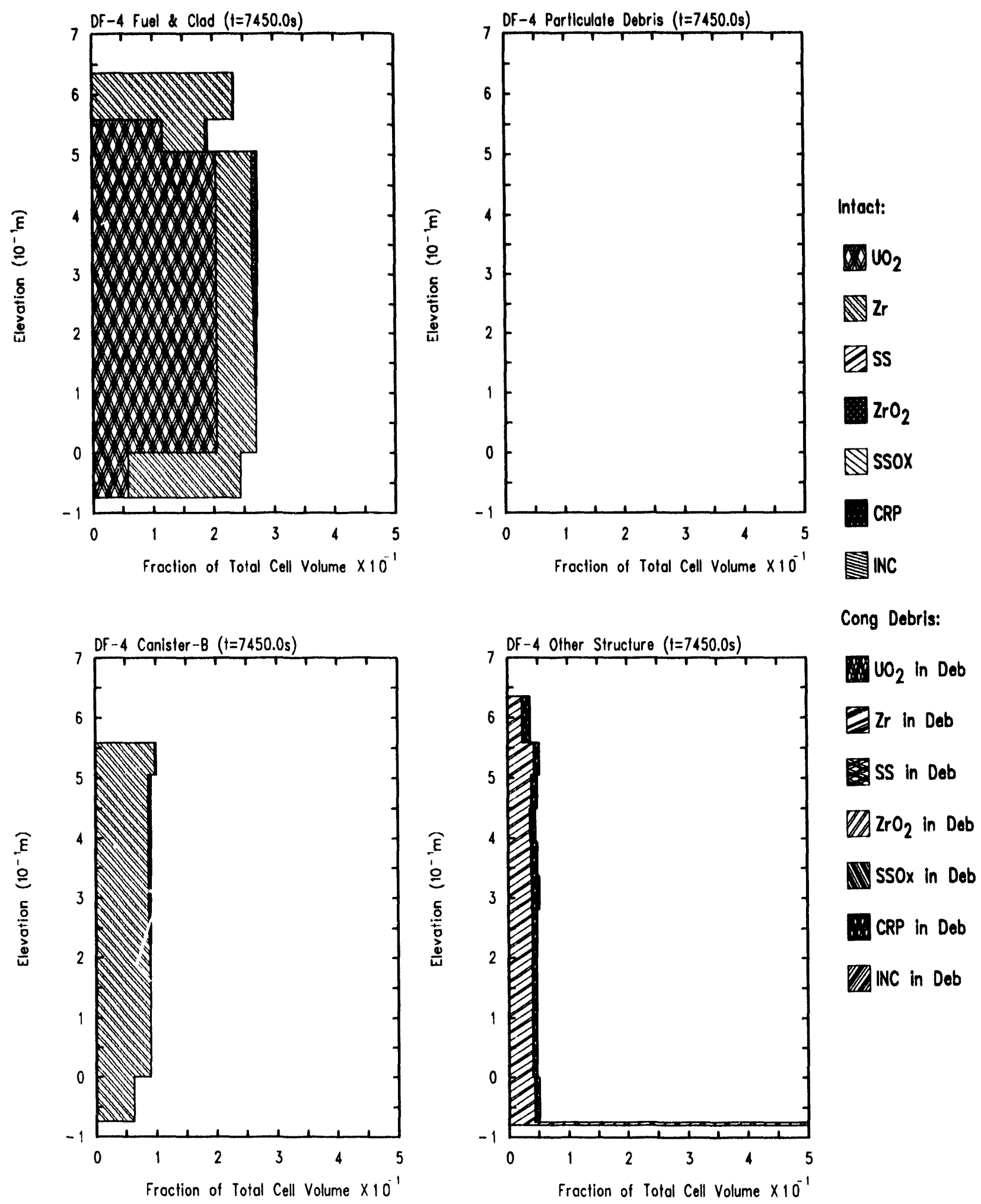

Figure 4.2.2. Locations of fuel and clad (upper left), particulate debris (upper right), canister-b (lower left) and other structure (lower right) component materials in DF-4 at 7450 seconds. 

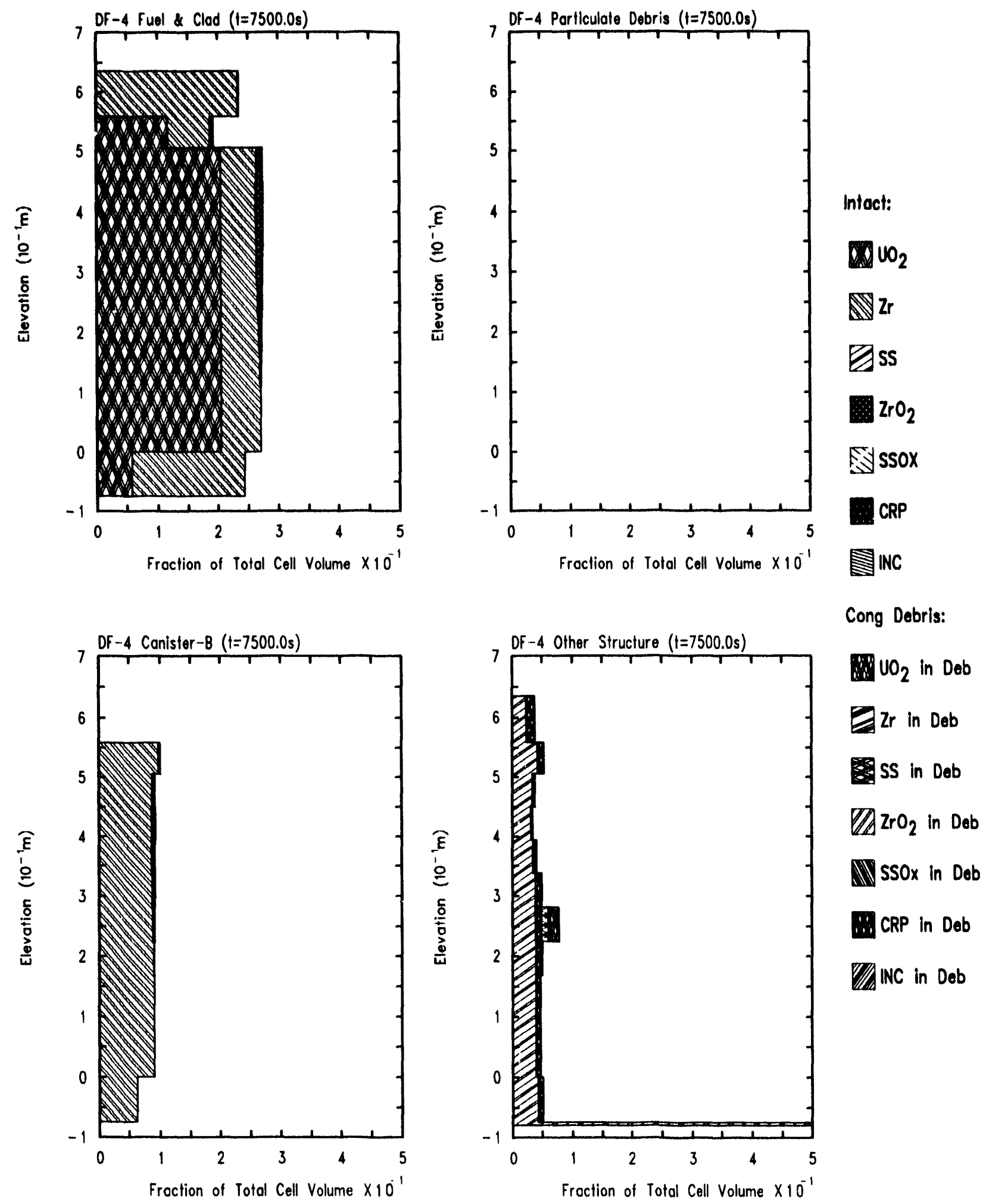

Figure 4.2.3. Locations of fuel and clad (upper left), particulate debris (upper right), canister-b (lower left) and other structure (lower right) component materials in DF-4 at 7500 seconds. 

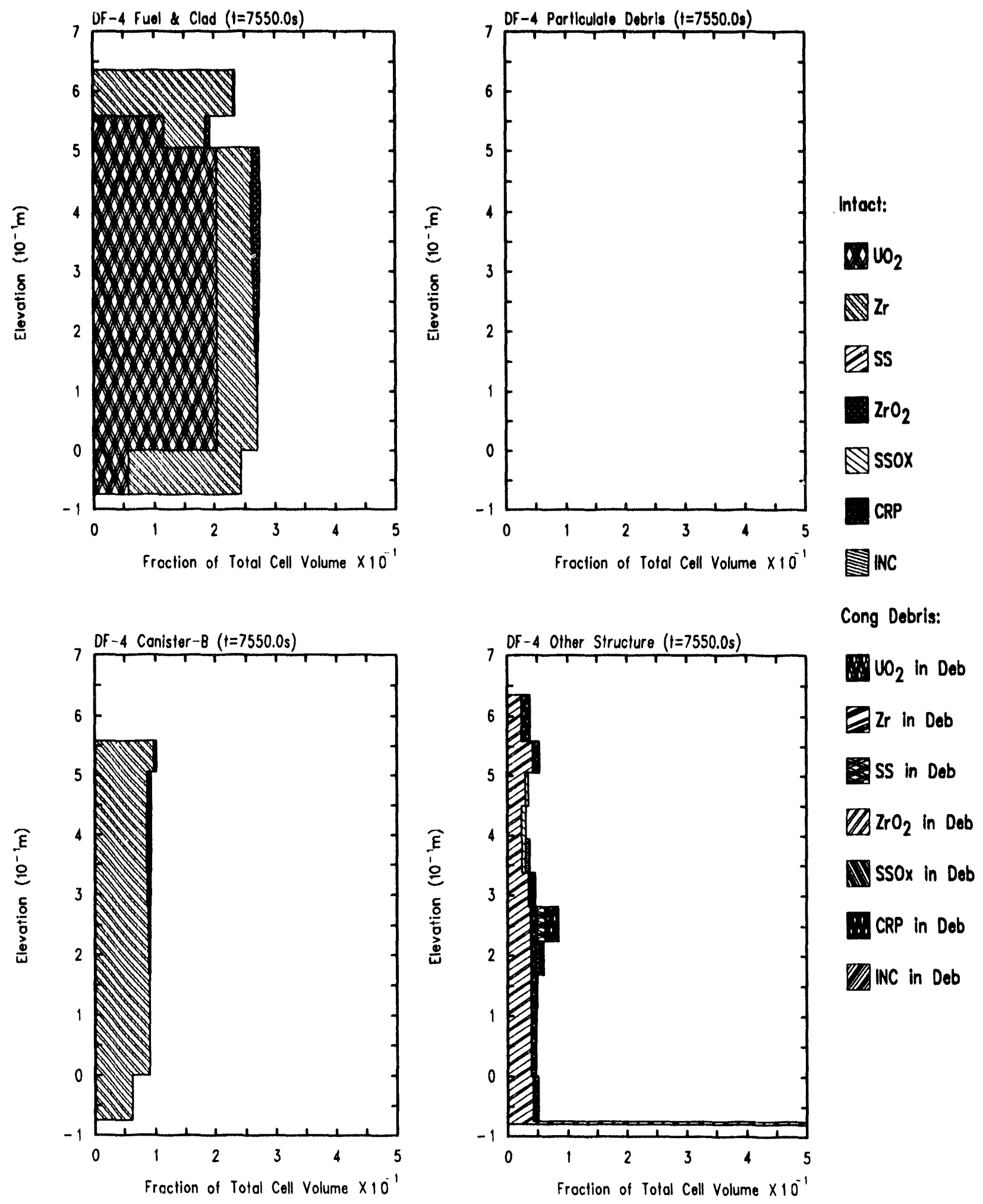

Figure 4.2.4. Locations of fuel and clad (upper left), particulate debris (upper right), canister-b (lower left) and other structure (lower right) component materials in DF-4 at 7550 seconds. 

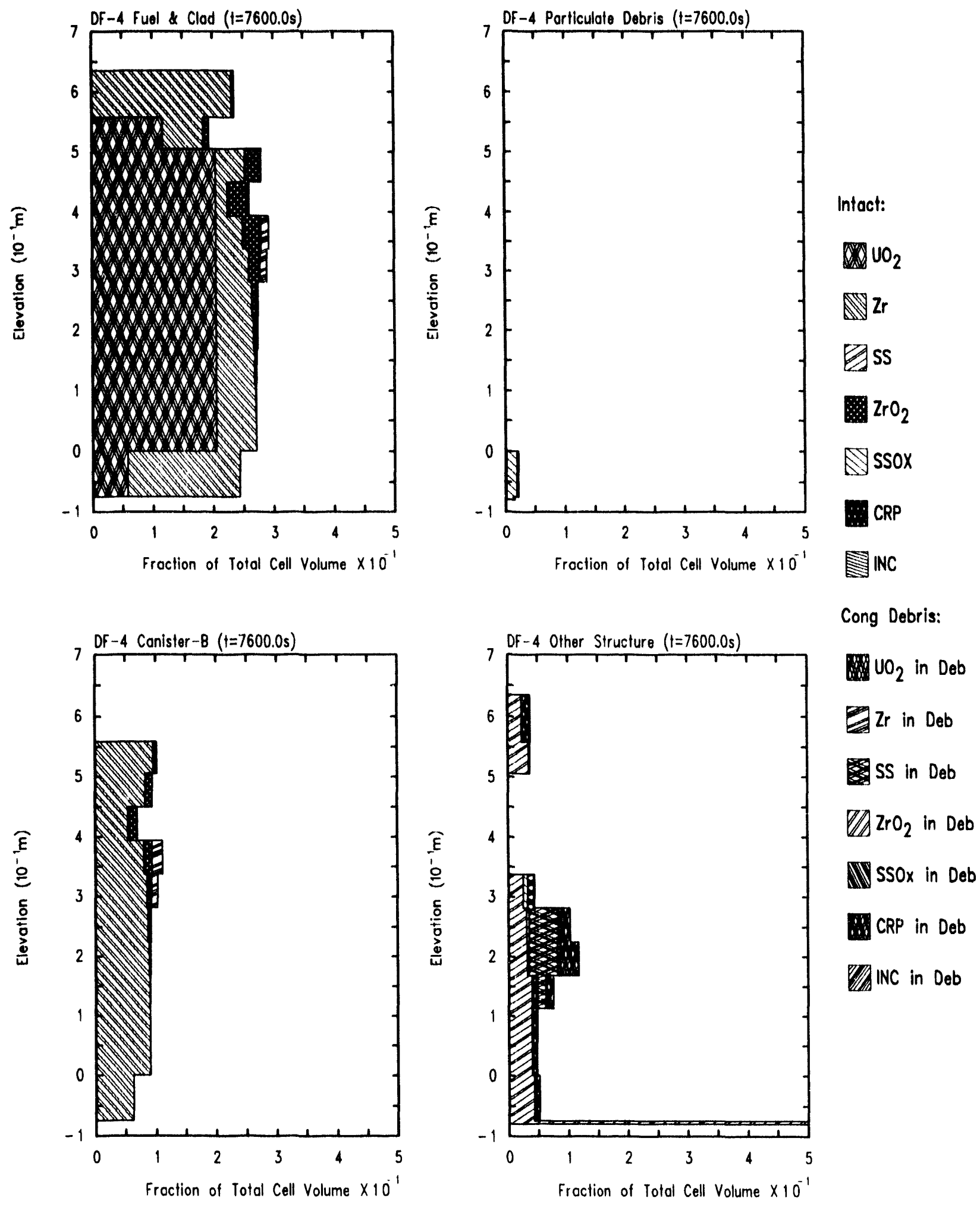

Figure 4.2.5. Locations of fuel and clad (upper left), particulate debris (upper right), canister-b (lower left) and other structure (lower right) component materials in DF-4 at 7600 seconds. 

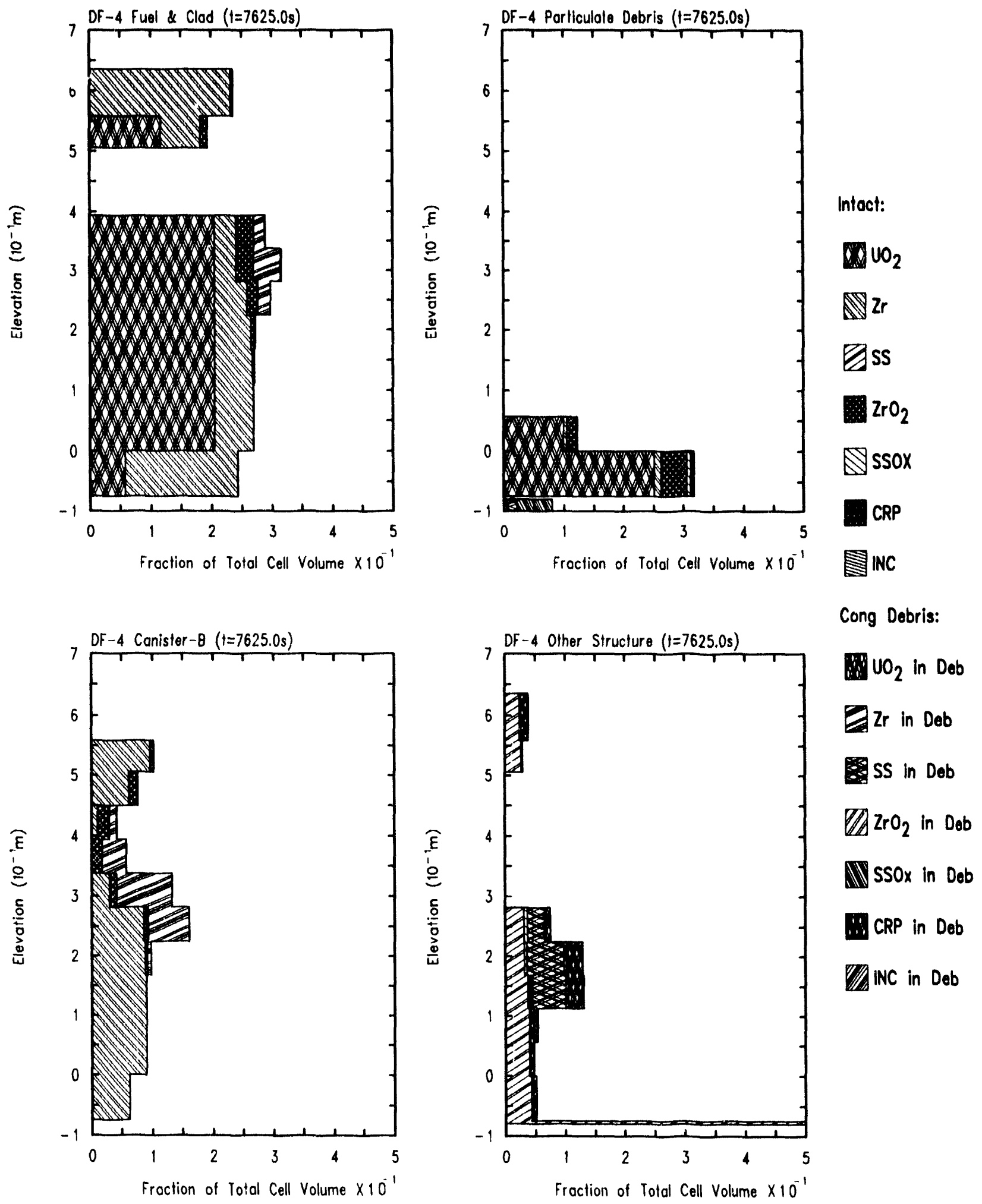

Figure 4.2.6. Locations of fuel and clad (upper left), particulate debris (upper right), canister-b (lower left) and other structure (lower right) component materials in DF-4 at 7625 seconds. 
9 (elevations 22.4 and $28.1 \mathrm{~cm}$, respectively). At 7500 seconds the $\mathrm{B}_{4} \mathrm{C}$ was almost entirely depleted from axial level 11 (elevation $39.3 \mathrm{~cm}$ ), but the eutectic reaction had not depleted noticeable amounts of $\mathrm{B}_{4} \mathrm{C}$ from any other axial levels. The canister and clad components were continuing to oxidize slowly at this time.

The relocation of $\mathrm{B}_{4} \mathrm{C}$ had speeded up by 7550 seconds, as shown in Figure 4.2.4 All of the $\mathrm{B}_{4} \mathrm{C}$ had relocated from axial levels 10,11 and 12 (elevations $33.7,39.3$ and $44.9 \mathrm{~cm}$, respectively) by this time. This shows the migration of the eutectic reaction front both up and down from the origination point in level 11.

Note that the $\mathrm{B}_{4} \mathrm{C}$ and stainless steel did not candle very far down the control blade before refreezing. This was in contrast to the early arrival of melt measured on the control blade at the $9.6 \mathrm{~cm}$ plane, shown in Figure 4.1.4. This may indicate a different candling heat transfer coefficient for the control blade materials than that used in the MELCOR base case input model. A sensitivity study on this parameter is discussed in Section 5.1.7.

Figure 4.2.6 shows that ly 7625 seconds the first fuel relocation had taken place. Note that the fuel had completely relocated from axial level 11 . This was due to the onset of rapid oxidation in that cell, which quickly depleted the cladding, leaving no support for the fuel pellets in that cell. The resulting debris relocated down to the core plate at axial level 2 (elevation $-7.6 \mathrm{~cm}$ ), which supported debris. Part of the cladding had also candled, again going only two or three levels before refreezing. The onset of rapid oxidation in the cladding drove the canister component to rapid oxidation as well. The largest amount of canister oxidation had taken place at axial level 11, but had also spread in both directions, like the eutectic reaction front in the control blade at earlier times. Candling of molten zircaloy in the canister component showed the same behavior as shown for $\mathrm{B}_{4} \mathrm{C}$ in the blade, where refreezing took place only two or three axial levels below the melting level. The rapid oxidation in the canister in turn drove the stainless steel in the control blade to melting and relocation, candling down a maximum of three or four axial levels.

Figures 4.2.2- 4.2.6 show two important features of these MELCOR calculations. First, although the control rod poison material began to relocate before the fuel material, it did not candle far before refreezing onto the control blade. This behavior was highly dependent on the candling heat transfer coefficient (see Section 5.1.7). Second, large scale melting and relocation seemed to be driven by fuel rod heatup and oxidation, and took place level by level instead of component by component. That is, all materials in a cell tended to relocate together before large scale relocation took place in other cells. This is also important for reactivity control. Note that the eutectic interaction front traveled faster than the oxidation front. The issue of fuel and poison separation for this problem is therefore highly dependent on the candling of control blade materials.

\subsubsection{Later Oxidation Subphase (7650-7700 seconds)}

The progression of melting and relocation in the test bundle at 25 second intervals is shown in Figures 4.2.6- 4.2.9. The behavior of the fuel and cladding was similar to earlier times, where the fuel material in axial levels relocated level by level, 
accompanied by a small amount of zircaloy candling.

The behavior of the canister and control blade was also similar. Melting and relocation progressed on a level by level basis, with $\mathrm{B}_{\mathbf{4}} \mathrm{C}$ and other materials candling only two or three cells down before refreezing. Note that the control blade melting and relocation led that of the fuel and clad, but the control poison material did not relocate as far as the fuel did. Thus, the fuel and control rod poison leap-frogged each other, but remained relatively close. This conclusion is based upon the behavior of the core plate, which remained intact during this phase of the experiment, and on the candling heat transfer coefficients.

There are two more interesting features to note in Figure 4.2.9. First is the existence of stainless steel oxide in the particulate debris component at axial level three (elevation $-7.5 \mathrm{~cm}$ ). This material originated in the other structure component at axial level 13 (elevation $50.5 \mathrm{~cm}$ ), the level containing the upper spacer region of the fuel. This was the result of the stainless steel at this level melting and having no material in cells below on which to candle. In this situation, MELCOR put the candling material into the particulate debris component. Second, the canister at axial levels seven (elevation $16.8 \mathrm{~cm}$ ) and above had significant amounts of zircaloy in the conglomerate debris, but little $\mathrm{ZrO}_{2}$. This indicates that the zircaloy was not oxidizing very quickly in the canister at these levels. This was probably due to the oxide layer that had formed on the canister, above the conglomerate debris layer. It may also have been due to steam starvation as a result of debris oxidation farther down in the debris bed.

\subsubsection{Final State}

The final calculated state of the test bundle is shown in Figure 4.2.10, at 8100 seconds. This was the time when the ACRR was shut down (see Figure 2.2.1). This can be compared to photos resulting from the PIE, shown in Figures 4.2.11- 4.2.14 [5], for the $95 \mathrm{~mm}, 133 \mathrm{~mm}, 238 \mathrm{~mm}$ and $308 \mathrm{~mm}$ axial levels.

At $95 \mathrm{~mm}(9.5 \mathrm{~cm})$, the PIE showed that most of the refrozen zircaloy inside the test bundle was left unoxidized, with some oxidation taking place in the outer portions of the bundle. There were also large amounts of stainless steel refrozen at the $95 \mathrm{~mm}$ level and below, due to the early candling of control blade eutectic mixture. The calculated state at this level in Figure 4.2.10 shows large portions of zircaloy remaining in the canister-b conglomerate debris and some in the particulate debris component. This material would correspond to the refrozen material shown in Figure 4.2.11. Figure 4.2.10 shows small amounts of control blade conglomerate debris but no intact material at this level; this is in contrast to the void filling the former position of the control blade showed in the PIE photo in Figure 4.2.11. There is more $\mathrm{UO}_{2}$ and zircaloy at this level than shown by the PIE, indicating again that the control blade material was calculated by MELCOR to candle only a short distance in the test bundle before refreezing, rather than flowing all the way to the bottom of the bundle as it did in the experiment. This indicates that the default values for candling heat transfer coefficients for control blade materials (which were used in the MELCOR base case model) do not represent the materials in DF-4 very well. The 

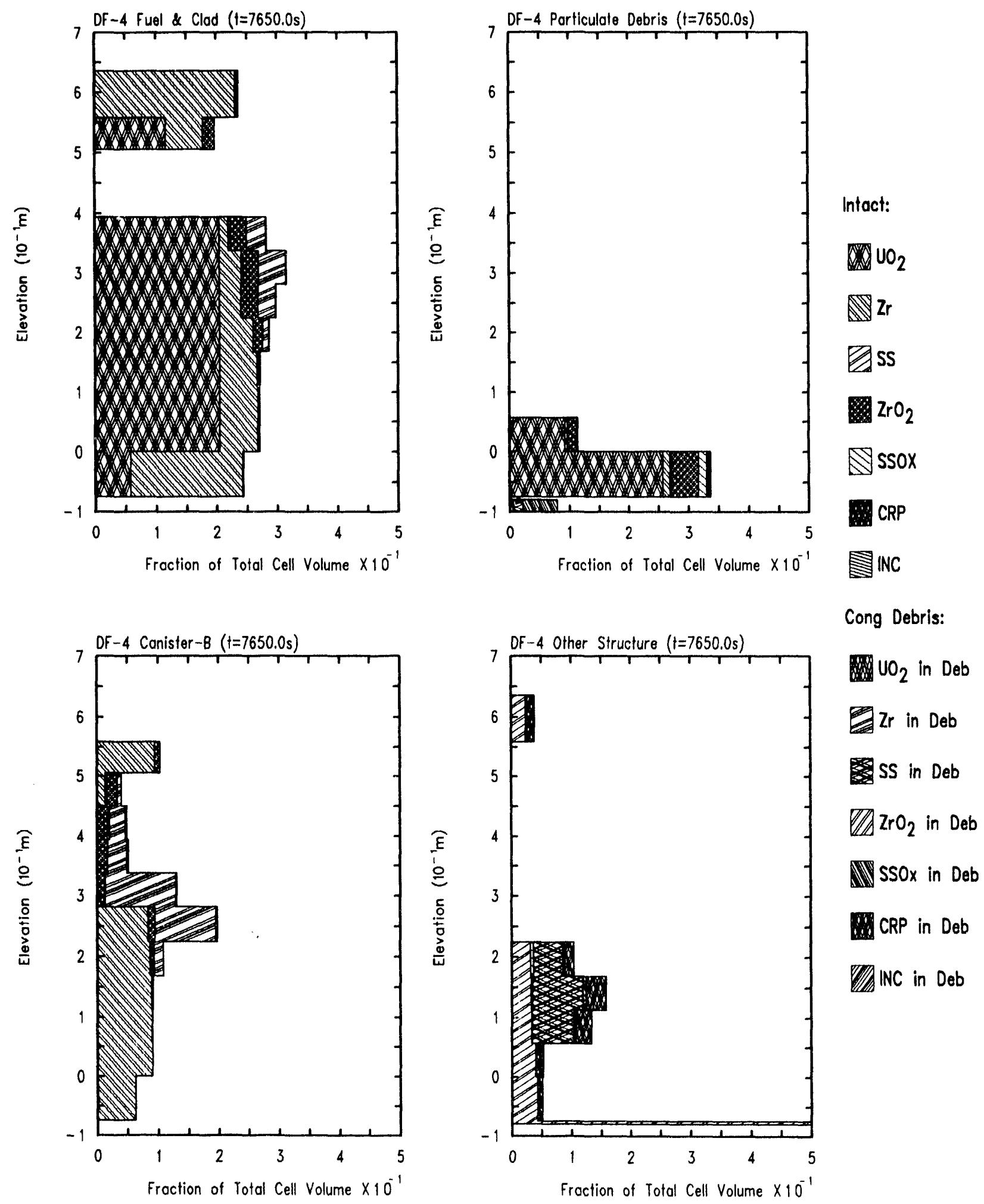

Figure 4.2.7. Locations of fuel and clad (upper left), particulate debris (upper right), canister-b (lower left) and other structure (lower right) component materials in DF-4 at 7650 seconds. 

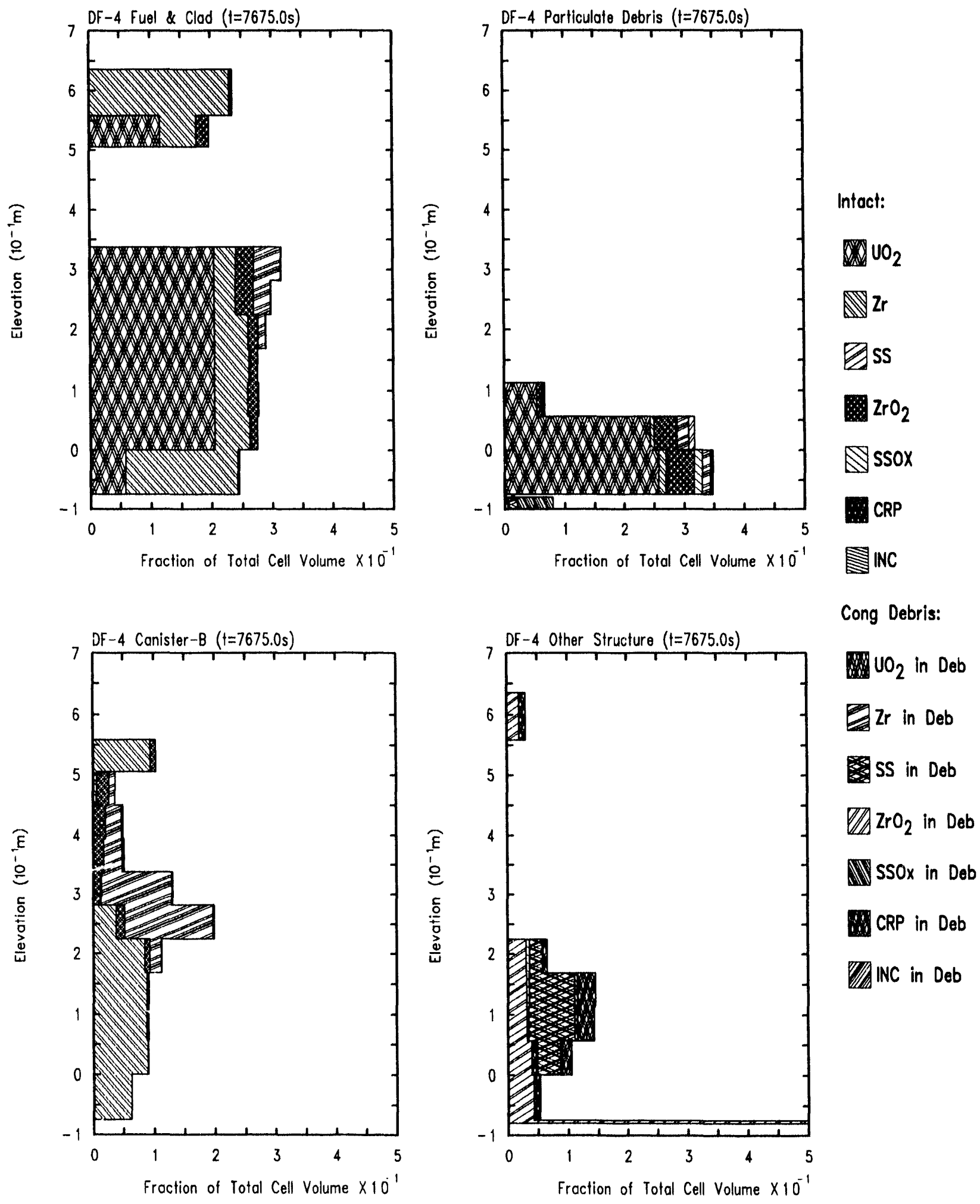

Figure 4.2.8. Locations of fuel and clad (upper left), particulate debris (upper right), canister-b (lower left) and other structure (lower right) component materials in DF-4 at 7675 seconds. 

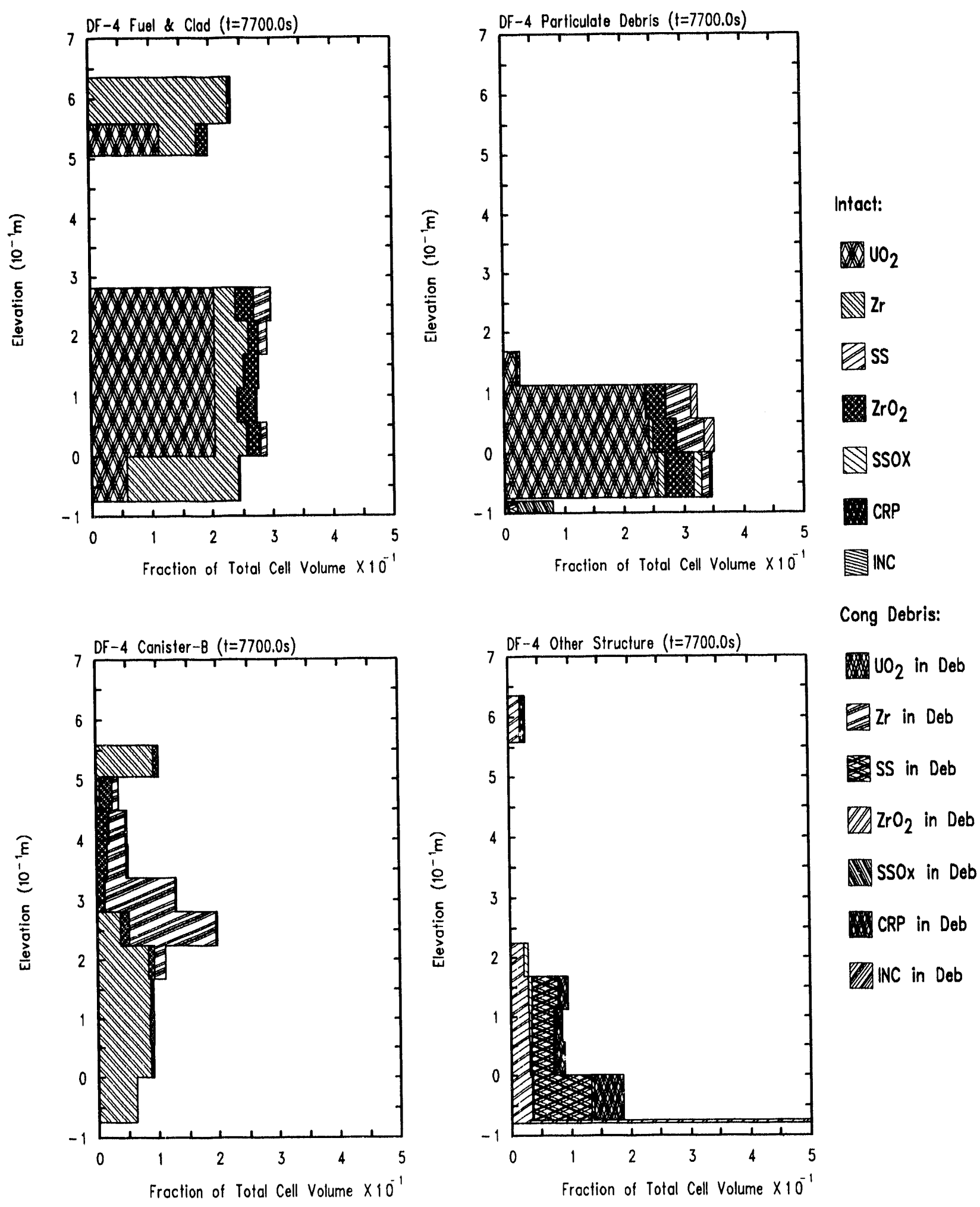

Figure 4.2.9. Locations of fuel and clad (upper left), particulate debris (upper right), canister-b (lower left) and other structure (lower right) component materials in DF-4 at 7700 seconds. 
control blade candling heat transfer coefficient defaults in MELCOR did not capture the rivulet-type candling shown by the video record of DF-4.

The debris at $133 \mathrm{~mm}(13.3 \mathrm{~cm})$ was very porous, as shown in Figure 4.2.12. This indicates the upper extent of debris in the test bundle. (In the next axial level shown in the PIE photos, the test bundle was largely void; this photo is not shown here.) MEICOR calculated the canister and control blade debris to end at approximately this level, neglecting the fuel debris.

Figure 4.2.13 shows some remnants of the canister and some material refrozen inside the canister area at $238 \mathrm{~mm}(23.8 \mathrm{~cm})$. 'The channel area between the remaining fuel rods was largely void. Figure 4.2 .10 shows $\mathrm{ZrO}_{2}$ remaining in the canister component at this level, corresponding to canister remnants. There were also substantial amounts of refrozen conglomerate debris on the canister at this level. This agrees well with the PIE results.

The PIE photo in Figure 4.2 .14 for the $308 \mathrm{~mm}(30.8 \mathrm{~cm})$ level shows some canister material remaining, and less debris material refrozen inside the canister.

At all levels, the PIE showed that the fuel rod pellets were mostly left standing. This was in contrast to the calculated state, where MELCOR relocated the fuel pellets after all the cladding had melted or oxidized. The stacking of fuel pellets has been observed in other core melt progression experiments, e.g. the CORA experiments [12]. There were various postulated reasons for this stacking, two of which were the internal Ti electrical heating elements inside the CORA fuel rods and the use of fresh instead of irradiated fuel in most of these experiments. However, there are no clear conclusions as to how the fuel pellets would behave during a real nuclear power plant accident. Therefore, we make no recommendations concerning the adjustment of fuel relocation parameters, except to say that these parameters should be investigated in a sensitivity study. A sensitivity study on core relocation parameters is reported in Section 5.1.8.

In summary, the distribution of debris materials in the test bundle was predicted by MELCOR with mixed success in comparison with the final state revealed during the PIE. The stainless steel was predicted to end up in the lower portions of the test bundle and there were large amounts of unoxidized zircaloy predicted in the debris, both in good agreement with PIE data. However, no fuel pellet stacking was predicted by MELCOR, resulting in a debris bed whose surface was higher than that observed in the PIE.

\subsection{Hydrogen Production}

The hydrogen production in DF-4 was measured using the hydrogen recombiner system designed specifically for this experiment. The measured total hydrogen production is compared to the calculated hydrogen production in Figure 4.3.1. Also shown is the total hydrogen production indicated by the hydrogen recombiner tubes, and an extrapolated production curve based on the final range of values for hydrogen production given by the PIE [6].

The calculated hydrogen production began much earlier than the measured production, building up to about 5 grams of hydrogen by 7550 seconds. The start of rapid 

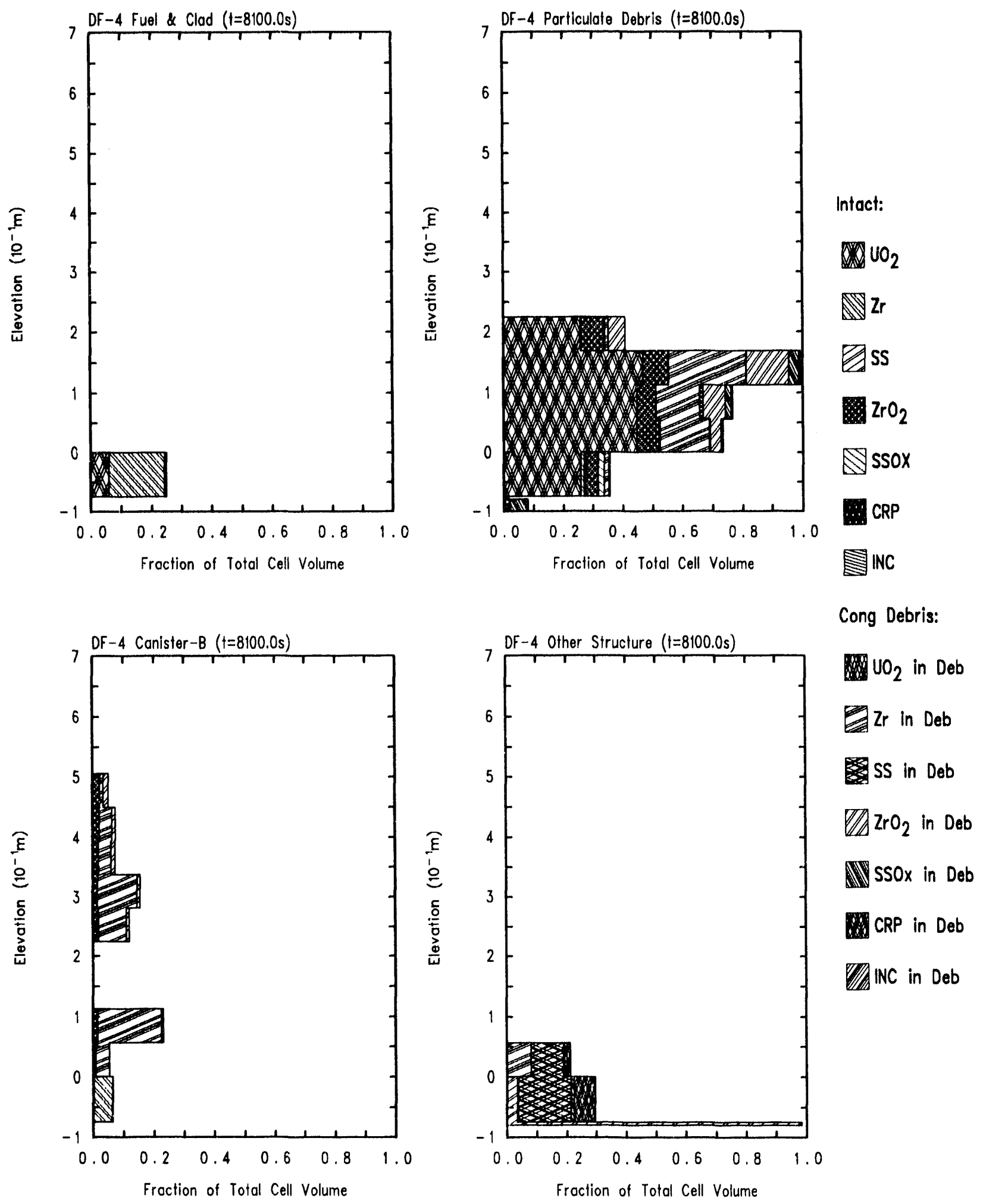

Figure 4.2.10. Locations of fuel and clad (upper left), particulate debris (upper right), canister-b (lower left) and other structure (lower right) component materials in DF-4 at 8100 seconds. 


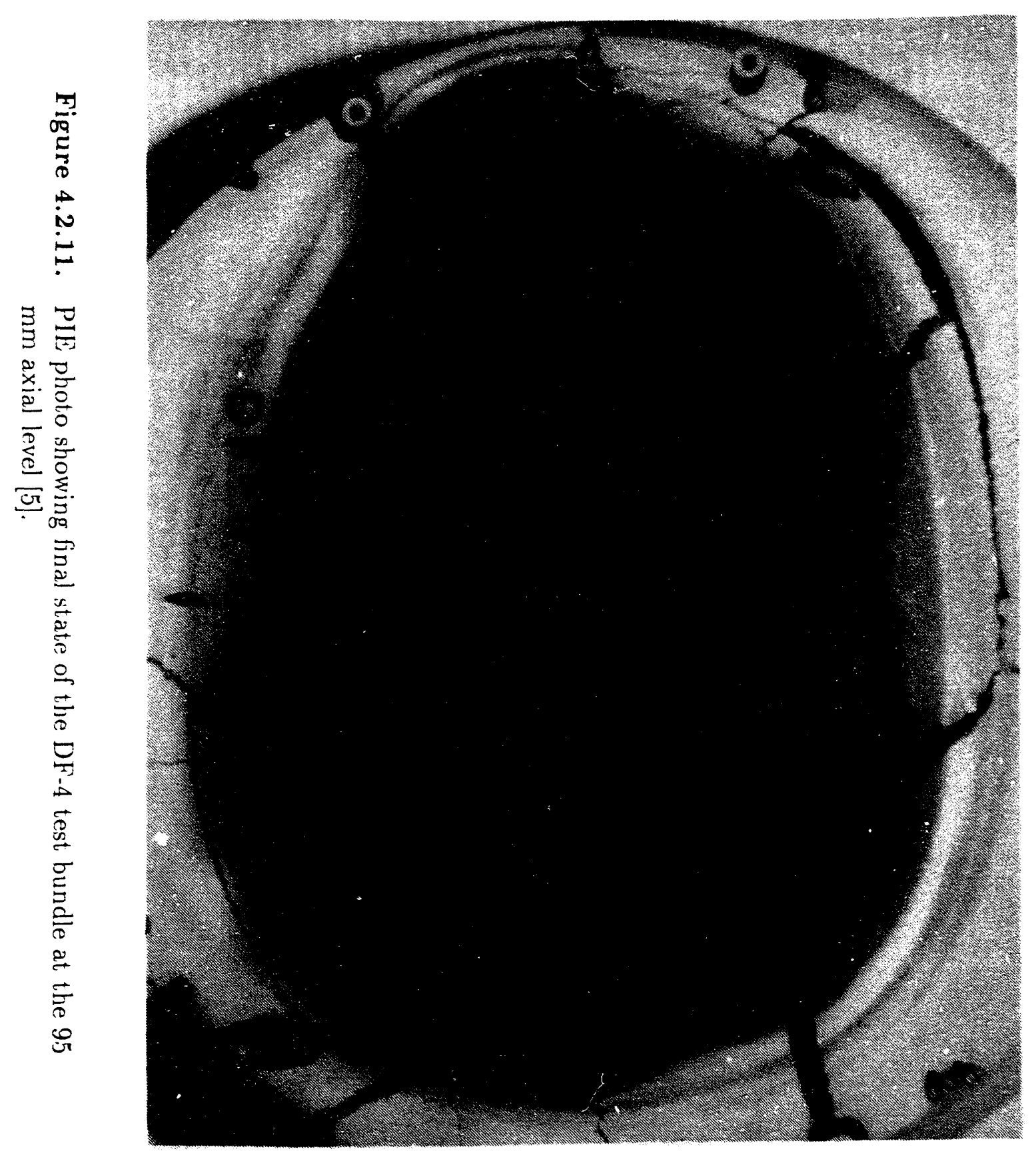




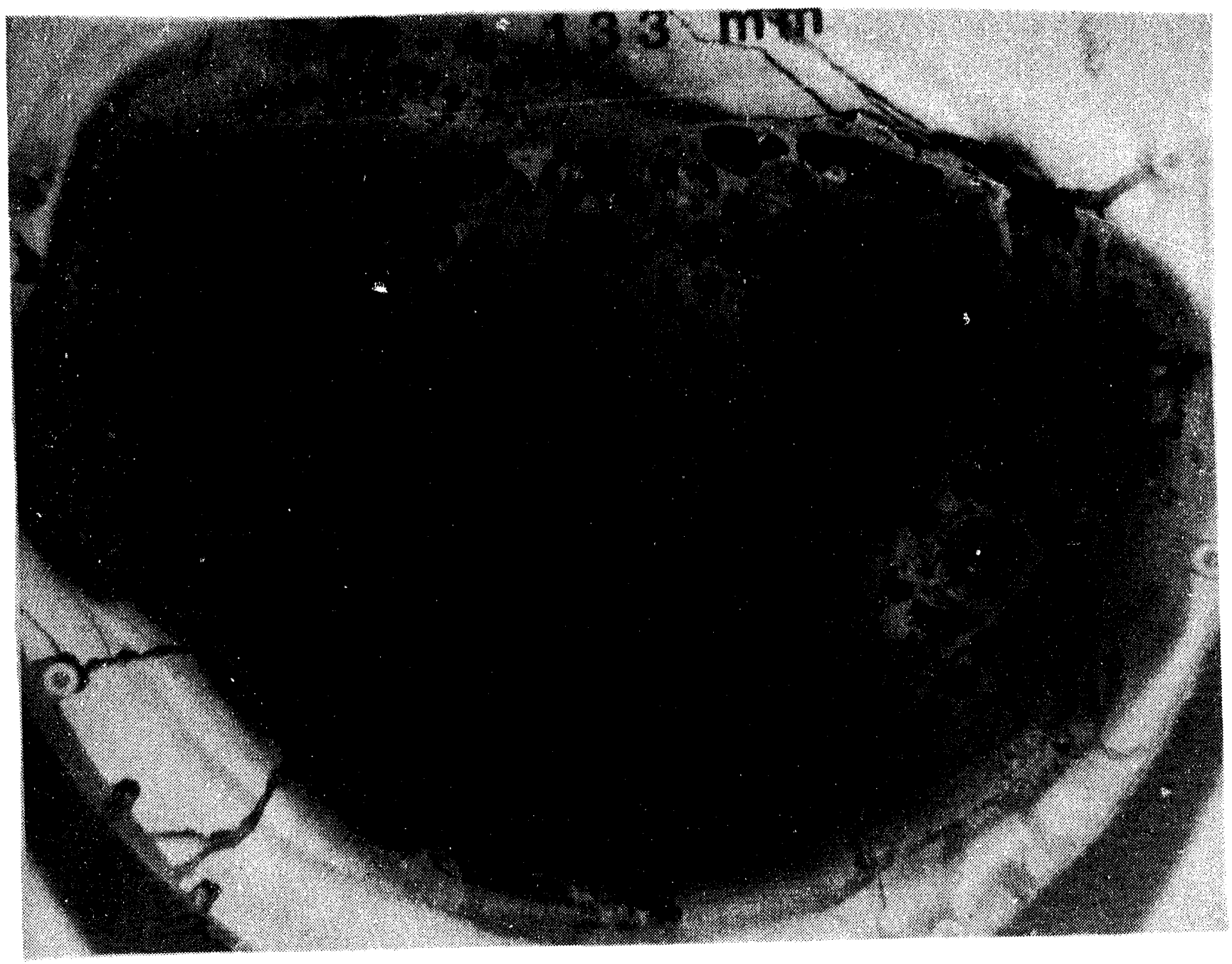

Figure 4.2.12. PIE photo showing final state of the DF-4 test bundle at the 133 mm axial level [5]. 


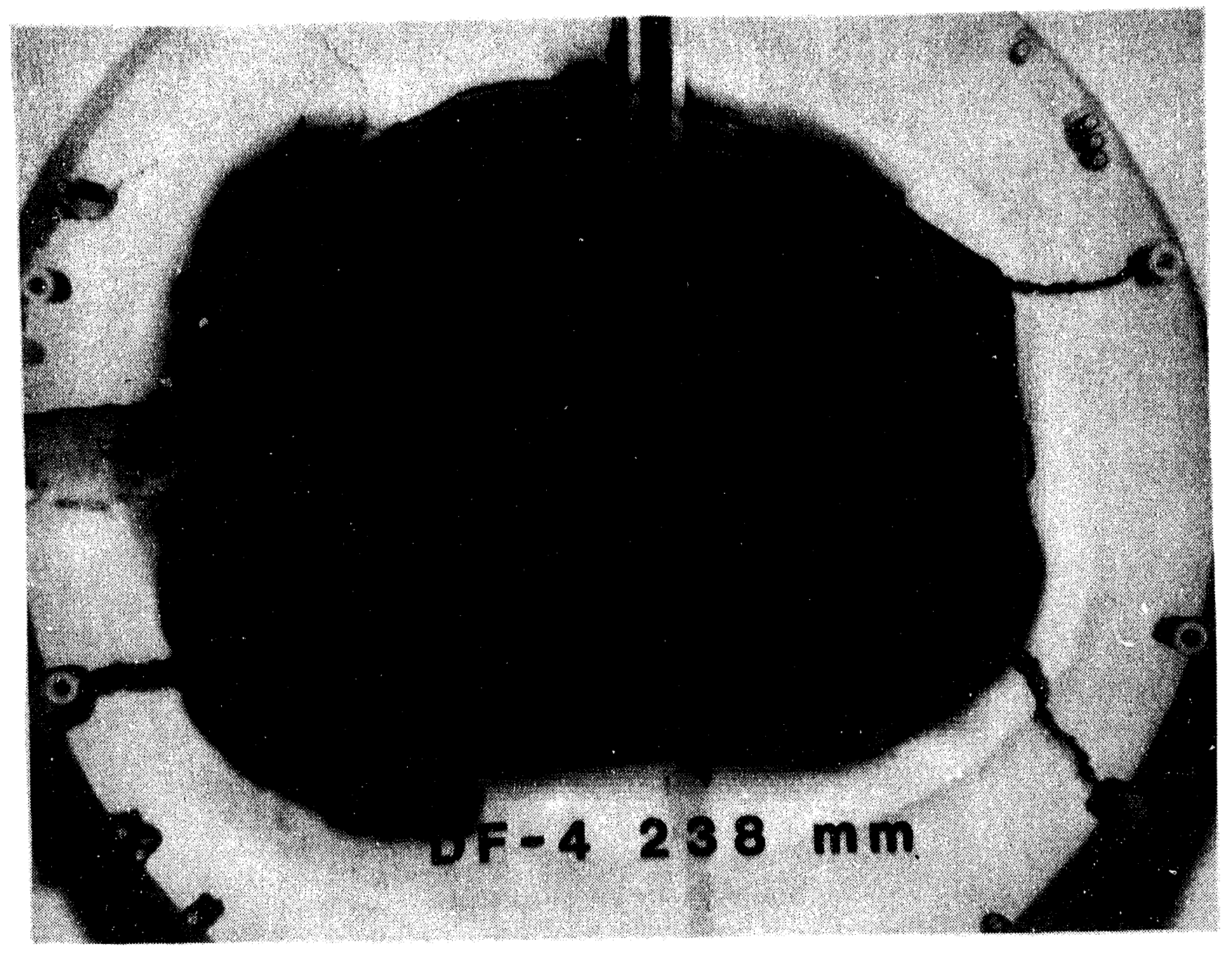

Figure 4.2.13. PIE photo showing final state of the DF-4 test bundle at the 238 $\mathrm{mm}$ axial level [5]. 


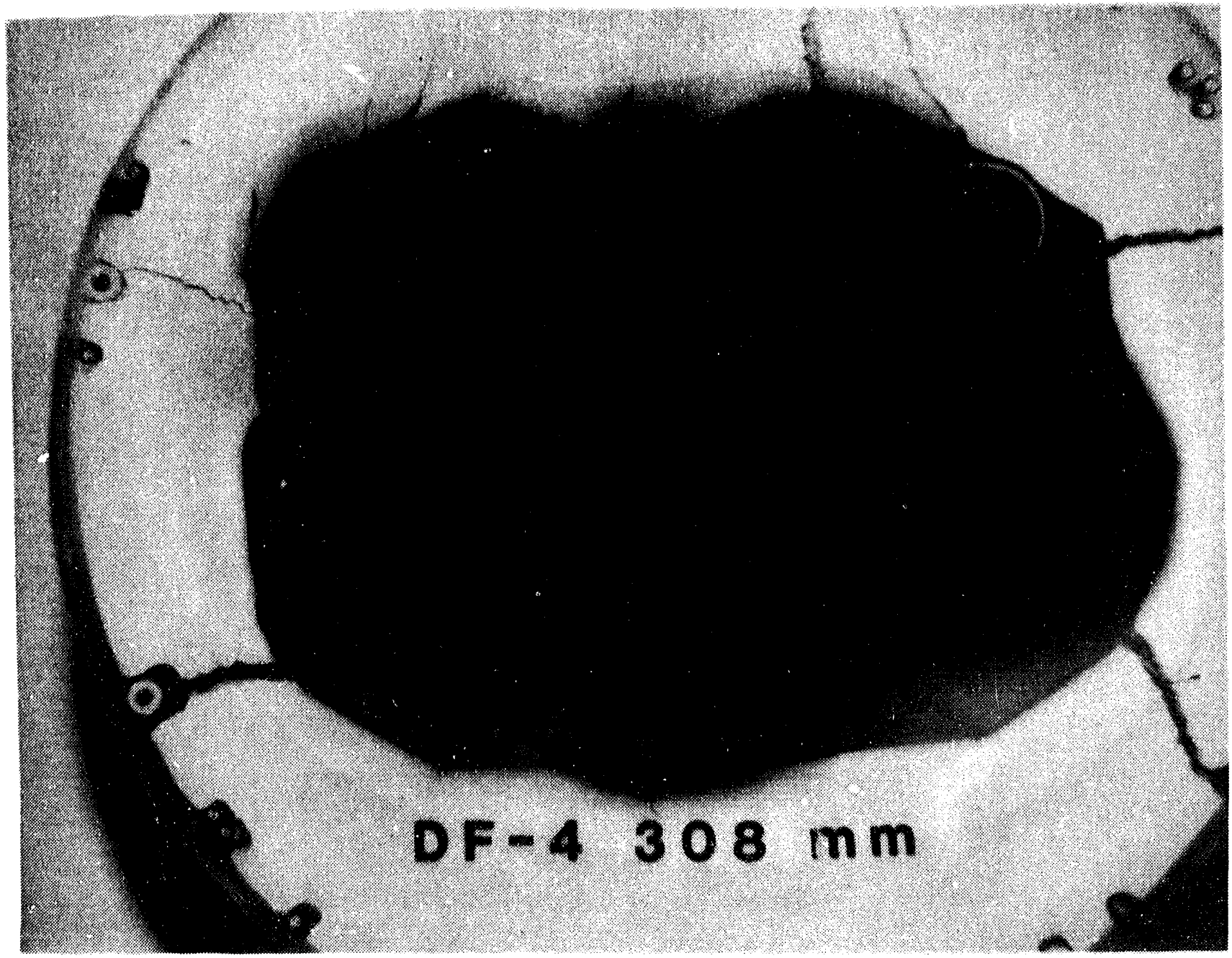

Figure 4.2.14. PIE photo showing final state of the DF-4 test bundle at the 308 mm axial level [5]. 
hydrogen production was very close to that measured in the experiment, but the production rate was initially higher than measured. The bulk of the calculated hydrogen production also took place early, compared to the measured hydrogen production. These results indicate that MELCOR may have over-predicted oxidation during the melting stage and under-predicted oxidation after debris had been formed. The same results for hydrogen production were also noted in the MELCOR model of the CORA13 experiment [13]. The total hydrogen production calculated by MELCOR was 36.4 grams, which was well within the range of 33-40 grams given by the DF-4 PIE [6].

The measured and calculated hydrogen production rates are shown in Figure 4.3.2. The maximum possible hydrogen production rate (corresponding to steam-starved conditions), based on an inlet steam flow of 0.88 grams/second, would be 0.098 grams/second. After the onset of rapid oxidation at about 7550 seconds, the production rate rose quickly to the steam-starved conditions. The calculated rate dropped along with the measured rate after the remainder of the core had relocated, but peaked again between 7800 and 8000 seconds. This was due to the uncovery of more unoxidized zircaloy by the eutectic dissolution of the canister. Note that the measured hydrogen production rate in Figure 4.3.1 started tailing off at about 7800 seconds. This indicates that the measured hydrogen production given by the recombiner tubes was not accurate after this time, since the measured production rate shown in Figure 4.3 .2 could not have resulted in the total hydrogen produced indicated by the PIE. Therefore, the peak in the hydrogen production rate predicted by MELCOR after 7800 seconds may have occurred during the experiment, despite the contradicting hydrogen recombiner data. 'This conclusion is reinforced by the hydrogen production rate calculated by the APRIL code, as described in Chapter 6. 


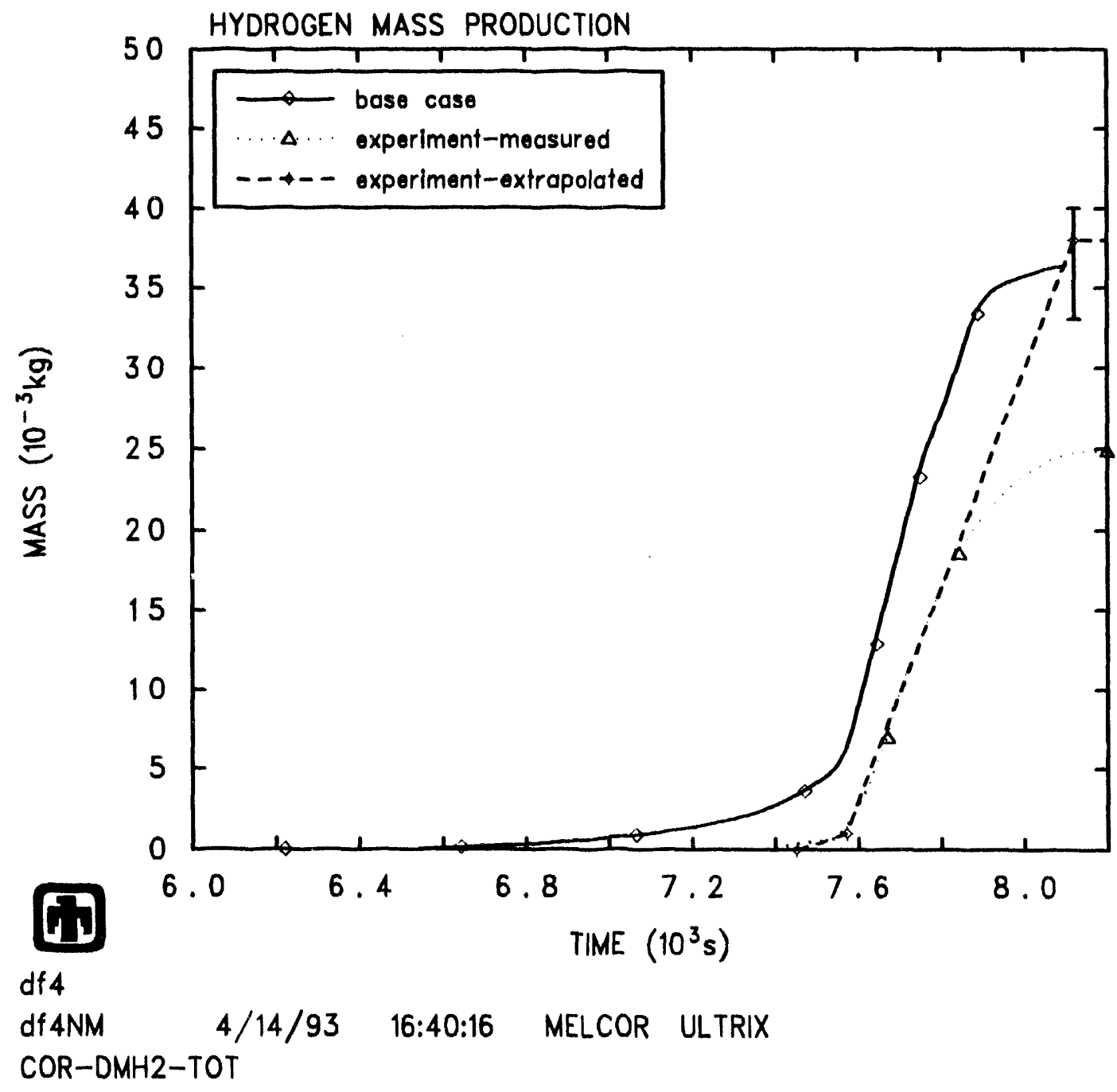

Figure 4.3.1. Calculated and measured integral hydrogen production in the DF-4 experiment. The final amount of the extrapolated production was given by the PIE. 


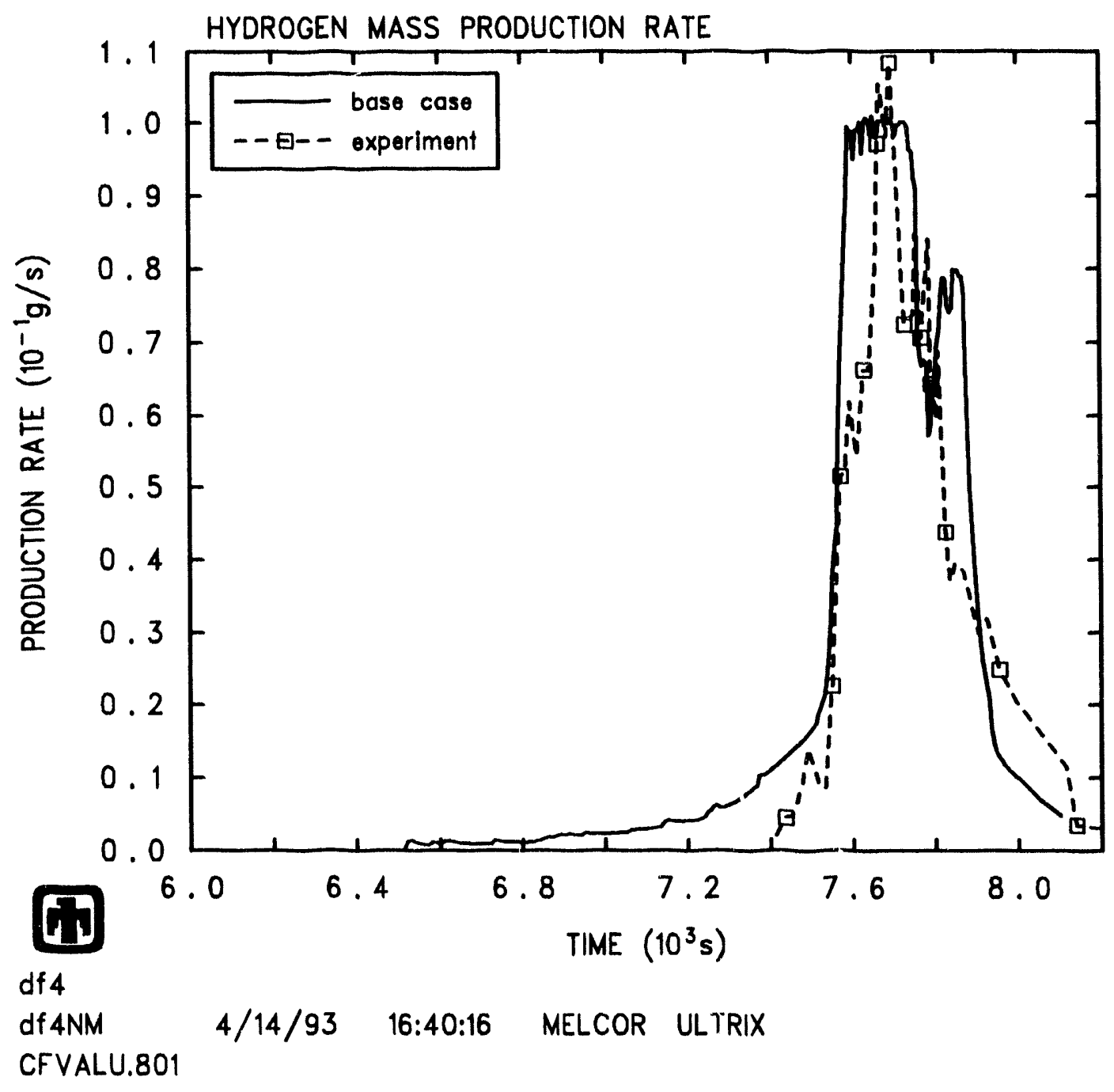

Figure 4.3.2. Calculated and measured hydrogen production rate in the DF-4 experiment. 


\section{Chapter 5}

\section{Sensitivity Studies}

A number of sensitivity studies were done to investigate the influence on results of varying all of the non-default options and some of the other input options that were used. Many of the non-default options used in the DF-4 base case model were the result of preliminary versions of these sensitivity studies. The focus of most of the sensitivity studies was the core package in MELCOR, because of the emphasis the DF-4 assessment placed on this package.

The studies reported in this chapter are grouped into eight main areas. These areas are: general core package input options, core package debris, oxidation and eutectic modeling, thermal/hydraulic initial and boundary conditions, heat structures package studies, COR and CVH nodalization for DF ' nd time step and machine dependency studies.

\subsection{General Core Package Studies}

Nine sensitivity studies on general MELCOR core package input options are reported in this section. The options studied are: convective heat transfer coefficients in the core package, core material properties, core component initial temperatures, core component view factors, canister mass and area partition, minimum component mass, candling heat transfer coefficients, minimum oxide shell thickness and boundary heat structure fluid temperature option. The studies are described in the order given.

\subsubsection{Convective Heat Transfer Coefficients}

For this sensitivity study, all the convective heat transfer coefficients in the COR package were increased and decreased by $50 \%$. This was done using sensitivity coefficient arrays 1212, 1214, 1221, 1222, 1231, and 1232.

The hydrogen production for the two variations is compared to that of the base case and to experimental values in Figure 5.1.1. Both increasing and decreasing the convective heat transfer coefficients decreased the amount of hydrogen generated during the experiment. The difference in production took place mostly after $7700 \mathrm{sec}-$ onds, which was after most relocation had taken place. This suggests that decreased 
production in the debris state had the most influence on final results. This conclusion is reinforced by comparing the cladding and control blade temperatures to base case and measured results. Figures 5.1.2 and 5.1.3 show that temperatures in the cladding and control blade, respectively, were not affected very much by variations in convective heat transfer coefficients. The largest variation was in the control blade during the test bundle equilibration subphase (6400-7200 seconds), when the control blade was being heated up as a result of heat transfer through the canister. The higher the heat transfer coefficient, the hotter the control blade was and the earlier blade failure occurred. The behavior observed here is similar to that observed in the LOFT LP-FP-2 MELCOR assessment [11], which also indicated nonmonotonic dependence of hydrogen production on core convective heat transfer.

\subsubsection{Core Package Material Properties}

Properties from the MATPRO materials properties package [9] were used in the base case model (the MATPRO package is used in the CONTAIN [14], MELPROG [15] and SCDAP/RELAP5 [16] codes). For this study, the material properties for zircaloy were changed back to the MELCOR default values. The most significant change made for this study was the decrease in zircaloy melting temperature from the MATPRO value of $2200 \mathrm{~K}$ to the MELCOR default value of $2098 \mathrm{~K}$. Material properties were adjusted using a combination of MP and TF package inputs.

The temperature response of all core components was almost identical, up to the point of zircaloy melting. Figure 5.1.4 shows that the cladding temperature in both calculations at the $36.8 \mathrm{~cm}$ plane was identical for this study, and the component failed at nearly the same time. Figure 5.1.5 shows that, when the MELCOR-default zircaloy melting temperature was used, the hydrogen production was decreased from 36.4 grams to 28 grams. The lower zircaloy melting temperature caused the cladding to melt and relocate downward, out of the oxidizing regions. This reduced the total mass of hydrogen produced from cladding oxidation.

\subsubsection{Core Component Initial Temperatures}

In the DF-4 MELCOR base case model, all core components were assumed to have initial temperatures of $480 \mathrm{~K}$. The core component temperatures at the beginning of the DF-4 experiment (at 6000 seconds) were actually in the range of $420-510 \mathrm{~K}$ (see Figure 3.2.4). For this sensitivity study, the temperatures of the fuel, cladding, canister and other structure components were set to the instrumented initial temperatures (the fuel component temperature was set equal to the cladding component temperature). Initial component temperatures were set on record CORijj03.

After the first few hundred seconds, the component temperatures calculated for this study were identical to the base case temperatures. All other quantities calculated by MELCOR for this study were also the same as the base case values. 


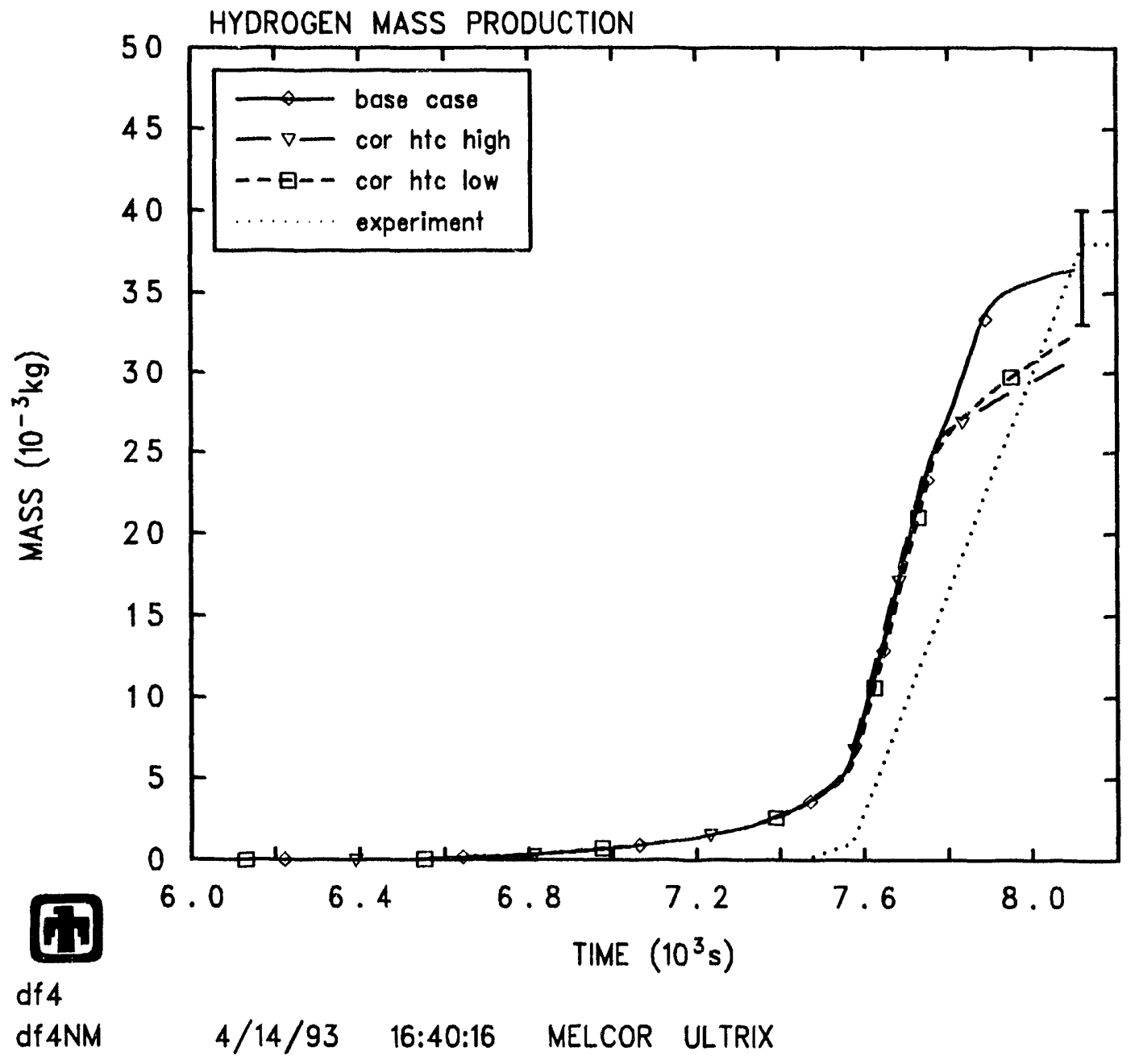

Figure 5.1.1. Hydrogen production for variations on the core convective heat transfer coefficients, compared to base case and measured values. 


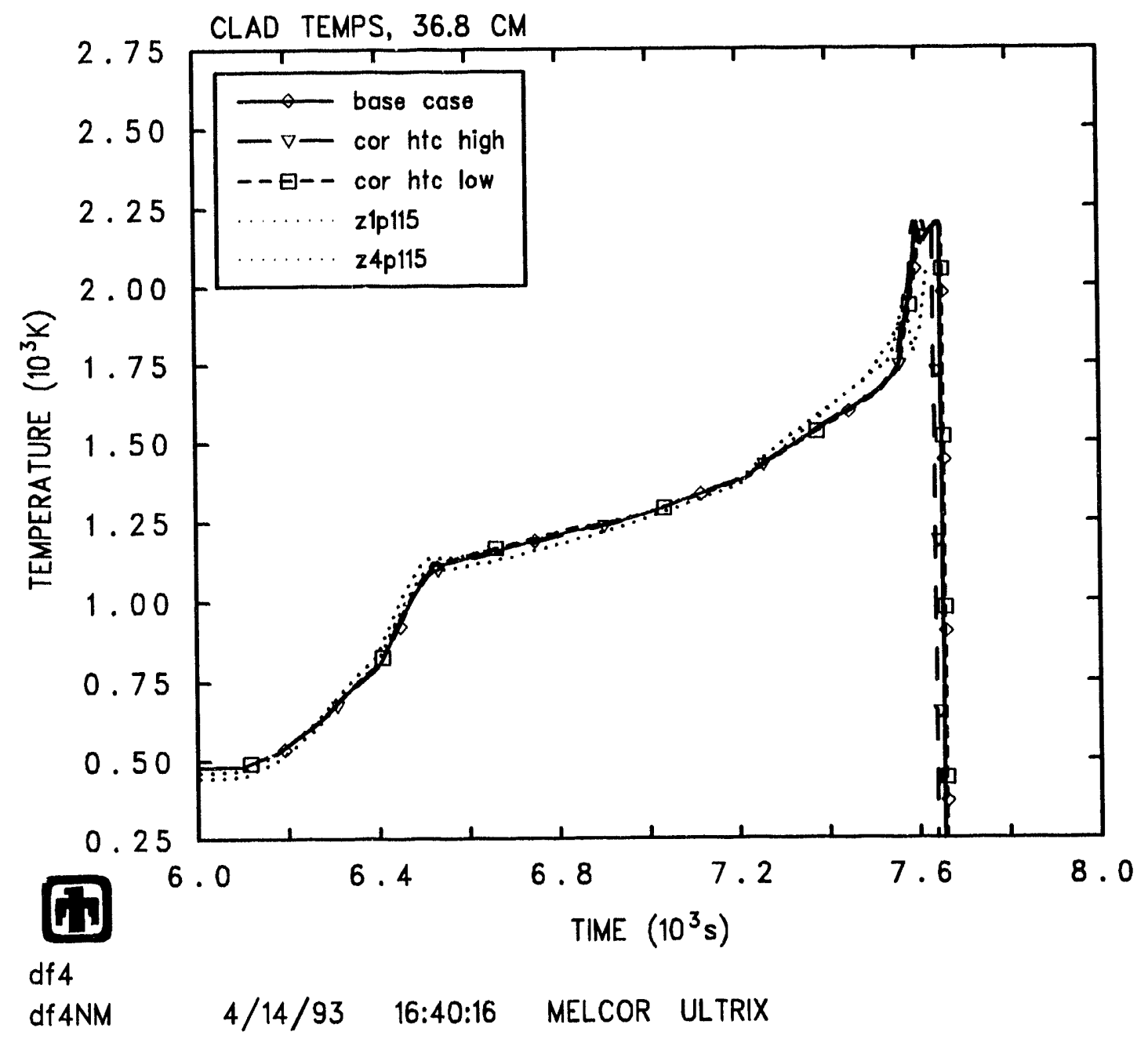

Figure 5.1.2. Cladding temperatures at the $36.8 \mathrm{~cm}$ plane for variations on the core convective heat transfer coefficients, compared to base case and measured values. 


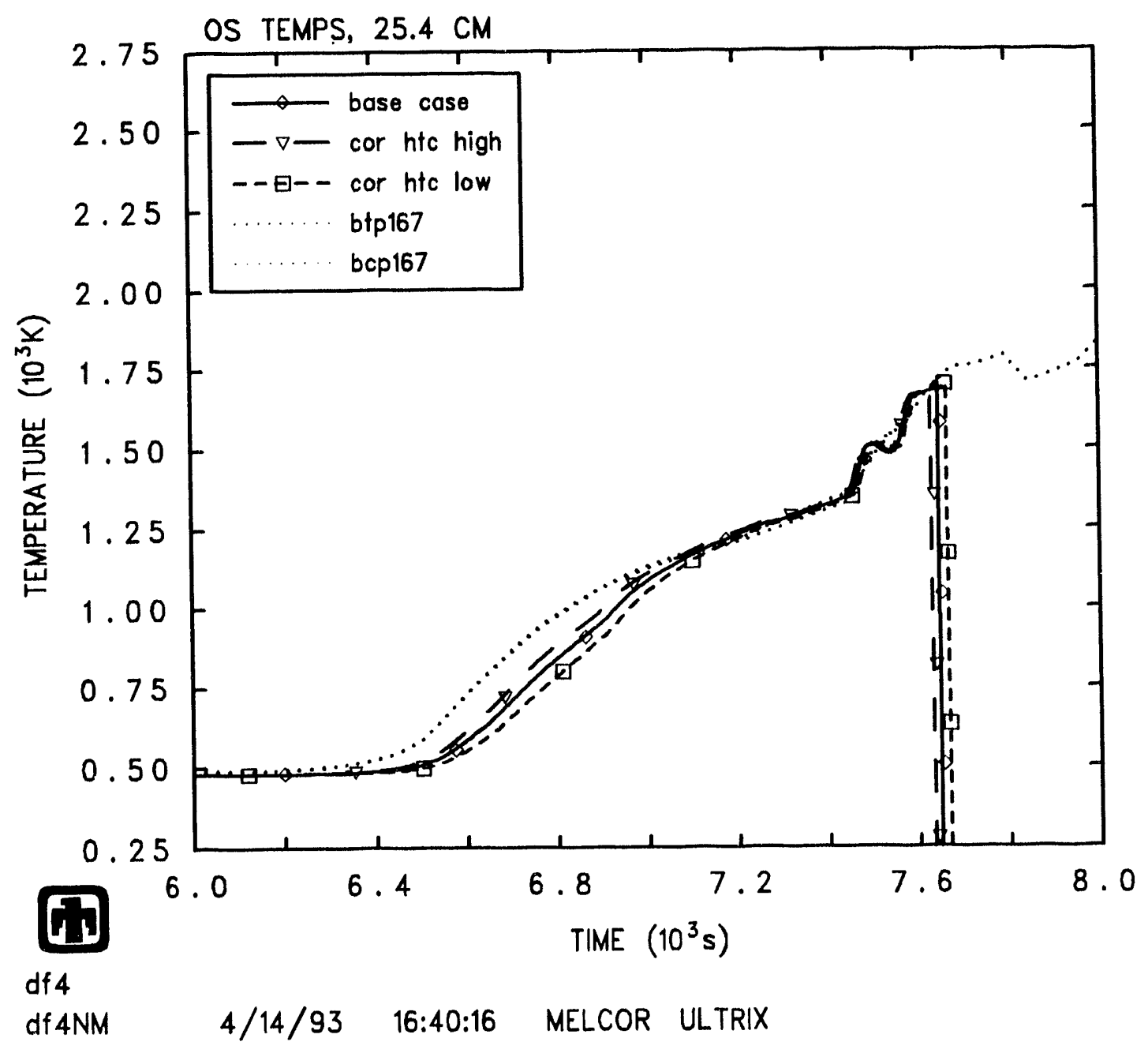

Figure 5.1.3. Control blade temperatures at the $25.4 \mathrm{~cm}$ plane for variations on the core convective heat transfer coefficients, compared to base case and measured values. 


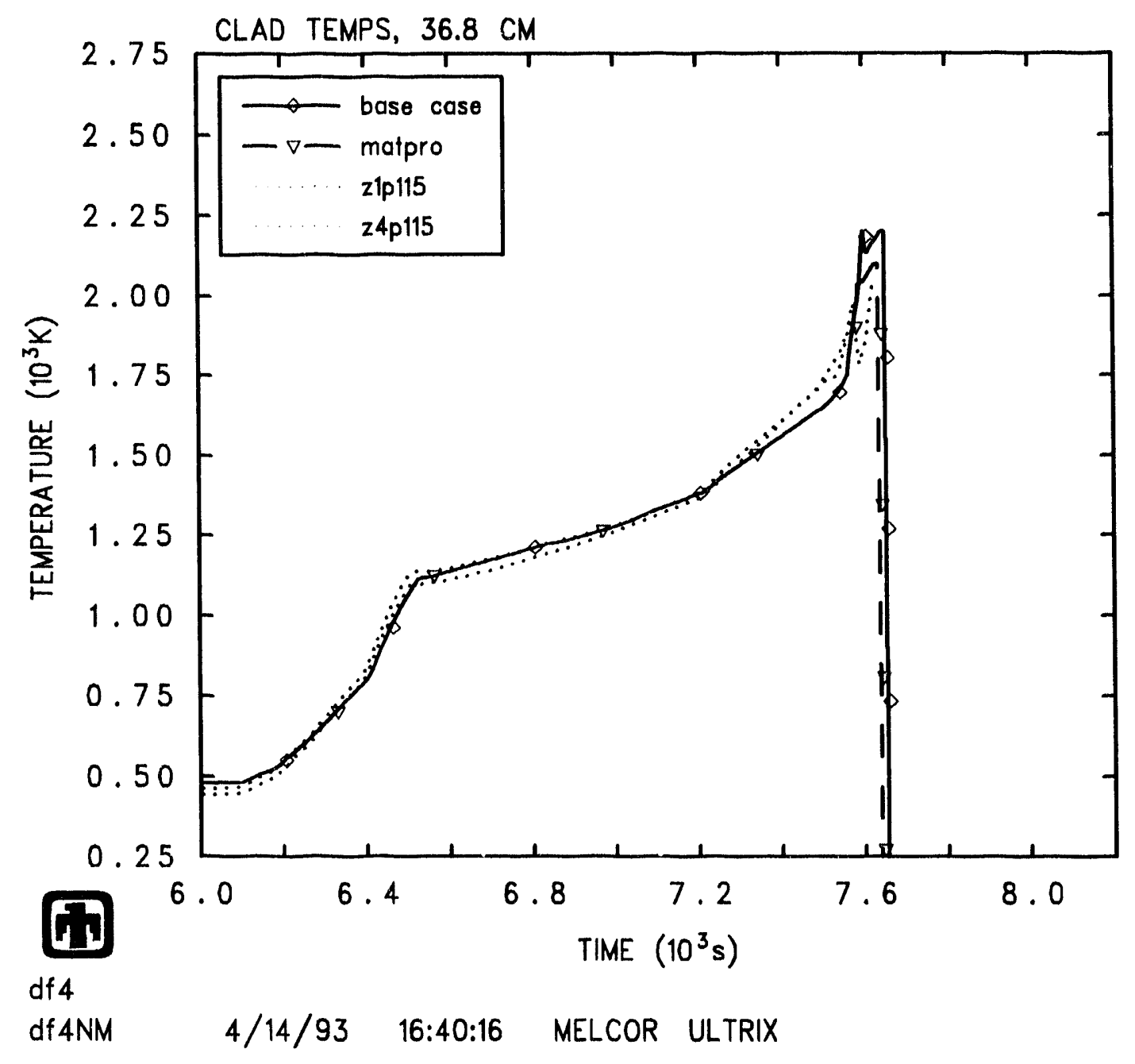

Figure 5.1.4. Cladding temperatures at the $36.8 \mathrm{~cm}$ plane for the MATPRO sensitivity study, compared to base case and measured values. 


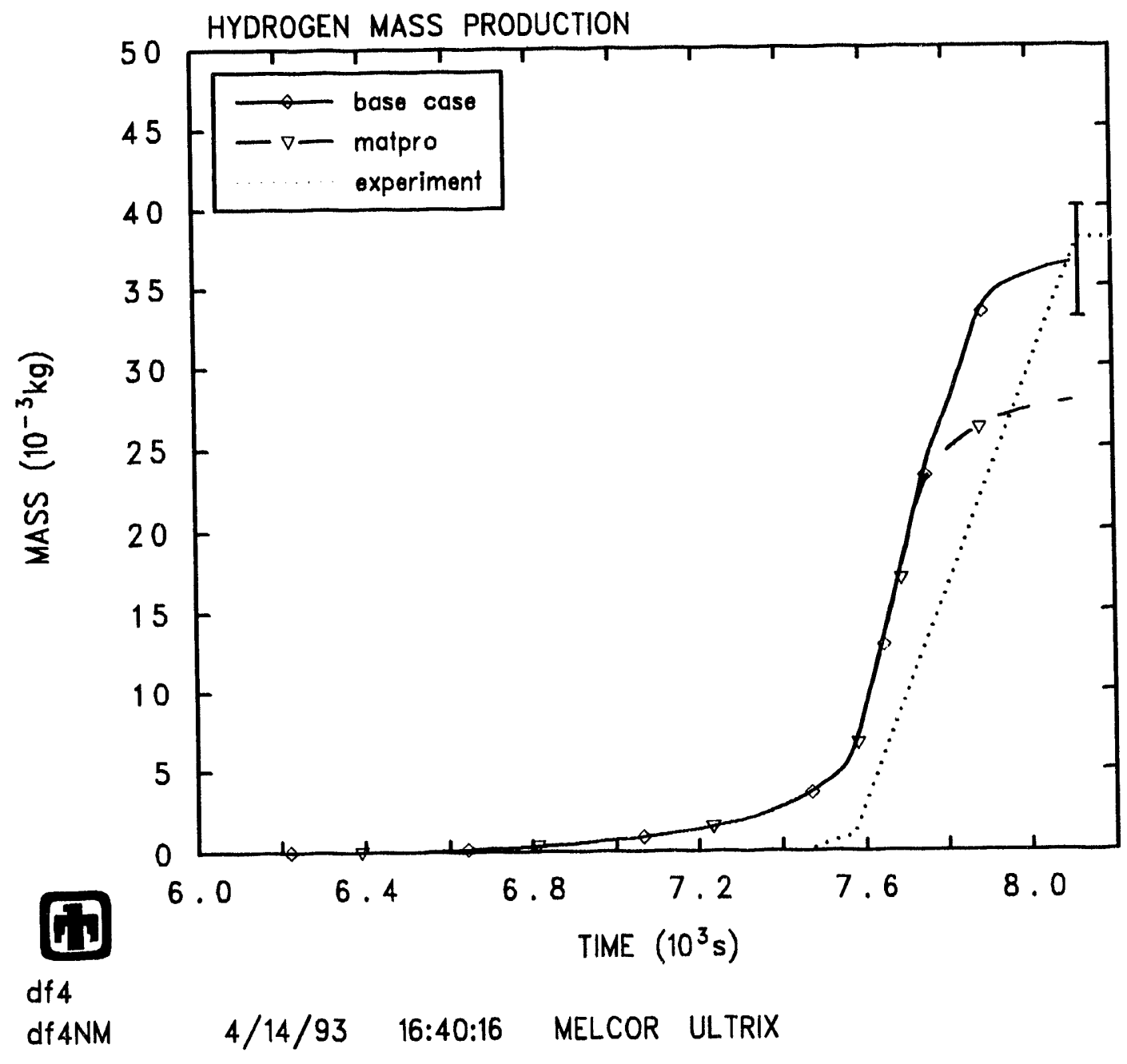

Figure 5.1.5. Hydrogen production for the MATPRO sensitivity study, compared to base case and measured values. 


\subsubsection{Core Component View Factors}

The core component view factors used in the MELCOR model for DF-4 were very important in determining heat losses to the insulating shroud outside the test bundle. The test bundle for DF-4 was designed to have high radiation heat transfer between the fuel rods and the canister, which was an important factor in heating up the control blade and starting the $\mathrm{B}_{4} \mathrm{C}$-stainless steel eutectic reaction. The view factors used in the DF-4 base case model were derived from analytical view factor calculations, and are given in Table 3.1.1. Three cases were run for this study, two cases with core package view factors that were $50 \%$ and $150 \%$ of the base case view factors, and a case using the MELCOR default core component view factors. View factors are input on record COR00003, and have default values of 0.25 .

The cladding temperatures at the $25.4 \mathrm{~cm}$ plane calculated for this study are shown in Figure 5.1.6. This shows that indeed the radiation view factors did have some influence on the temperature results. Increasing and decreasing the view factors had the expected effect on the cladding; increasing view factors to $150 \%$ of the base case values increased the radiation heat transfer from the fuel rods, which lowered the fuel rod temperatures and delayed cladding failure. Decreasing view factors to $50 \%$ of the base case values had the opposite effect. The cladding temperature was unchanged below a temperature of $1200 \mathrm{~K}$.

Although at first it seems like the default view factors case should have behaved like the $50 \%$ case (since most view factors in the DF-4 MELCOR base case model were greater than the default MELCOR values), in reality it behaved like the $150 \%$ view factors case. This is because the cell-cell (radial) view factor in the $150 \%$ case was closer to the default value than that of the $50 \%$ case. Increasing this view factor decreased the cladding temperatures and delayed cladding failure. This effect offset the decrease in the canister-other structure view factor, which resulted in less heat transfer to the control blade.

The control blade temperatures at the $36.8 \mathrm{~cm}$ plane showed slightly different behavior in the heatup stage of the experiment. Figure 5.1.7 shows that increasing and decreasing the view factors decreased and increased control blade temperatures at this plane, respectively. The changes were only evident at temperatures above $1200 \mathrm{~K}$, similar to the cladding temperature results. Using the default view factors lowered the control blade temperature. This was not surprising, since the canisterother structure view factor was lowered from the base case value of 0.9999 to the default value of 0.25 . This resulted in less heat transfer to the control blade and later control blade failure.

The hydrogen production for this study is shown in Figure 5.1.8. Decreasing view factors and thereby increasing cladding temperatures tended to increase early production of hydrogen, while increasing view factors and decreasing cladding temperatures had the opposite effect. Although the timing of hydrogen production was affected, the total amount produced was the same for both cases, and was approximately 3 grams less than that produced in the base case calculation. These data may indicate some tradeoff between the oxidation of iscaloy in the cladding component versus the oxidation of zircaloy in the particulate debris component. The default view fac- 
tor case behaved in a similar manner as the $150 \%$ case, as it did with the cladding temperatures.

In general, there was a fair amount of sensitivity of final results to view factors, mostly in the timing of failure and of hydrogen production but also in the total amount of hydrogen produced. Note that the influence of view factors on core degradation modeling would probably be greater in the absence of continued nuclear heating, since nuclear heating in DF-4 tended to drive core degradation no matter what the radiation heat transfer was. In any case, the $\mathrm{COR}$ component view factors should always reflect the geometry being modeled.

\subsubsection{Canister Mass and Area Partition}

The COR package in MELCOR allocates canister mass to two components, designated canister and canister-b. The canister-b component is that part of the canister which is adjacent to the control blade, while the canister component is adjacent to other canisters. The separation of the canister mass into these two components affects the heat transfer to the control blade and to the radial boundary in the DF-4 model.

In the MELCOR base case model, all canister zircaloy was allocated to the canister-b component. This was done to more closely match the geometry of the DF-4 test bundle (see Figure 2.1.1), where the majority of the canister area was adjacent to the control blade. In this sensitivity study, the canister mass and area were split evenly between the canister and canister-b components (this is the default configuration in MELCOR). The canister area partition between canister and canister-b components is controlled by sensitivity coefficient 1501 .

The temperature response of the cladding at the $36.8 \mathrm{~cm}$ plane is compared with that of the base case in Figure 5.1.9. This shows that the default partition of canister mass resulted in earlier failure of the cla iding. Figure 5.1.10 shows the same results for the control blade at the $25.4 \mathrm{~cm}$ plane. The reason for this could be that allocating less canister mass to the canister-b component provided less of a heat sink between the fuel rods and the control blade, resulting in earlier control blade and cladding heatup and failure.

The overall hydrogen production for this study was almost identical to the base case value, but was generated slightly earlier in the problem. This was consistent with the temperature behavior.

The final material distributions for the canister and canister-b components for this sensitivity study calculation are shown in Figure 5.1.11. These component distribution plots can be compared with the final base case canister-b component material distribution shown in Figure 4.2.10. This figure indicates that using the default partition of canister mass resulted in slightly less total canister mass renaining in the middle of the test bundle and slightly more refrozen zircaloy at the bottom of the test bundle at the end of the problem. This suggests that the canister and canister-b components melted faster in this sensitivity study calculation, and relocated to the bottom of the core.

This study indicates that there were slight differences in final material locations due to the allocation of all canister mass to the canister-b component, while the 


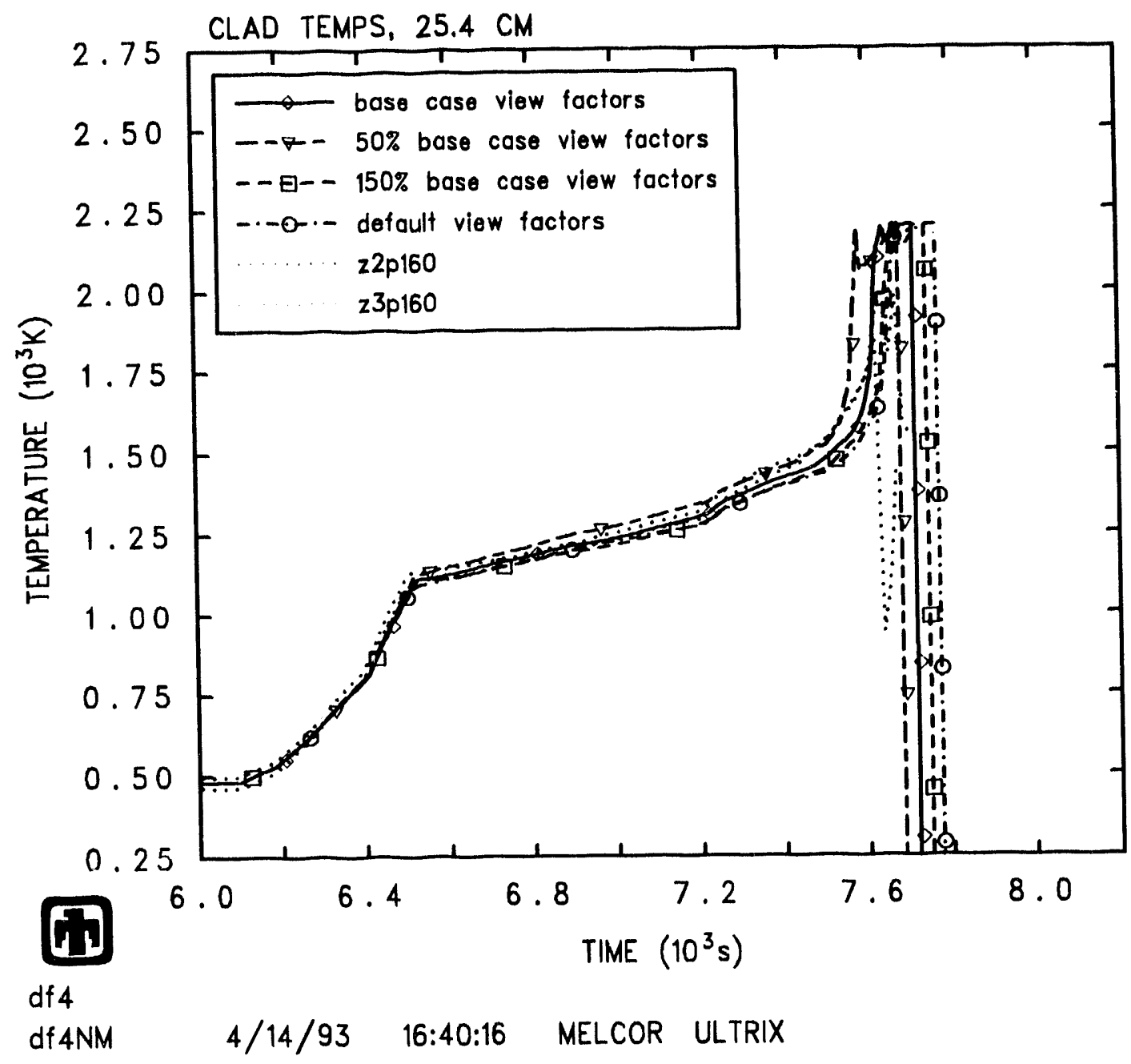

Figure 5.1.6. Cladding temperatures at the $25.4 \mathrm{~cm}$ plane for the radiation view factor sensitivity study, compared to base case and measured values. 


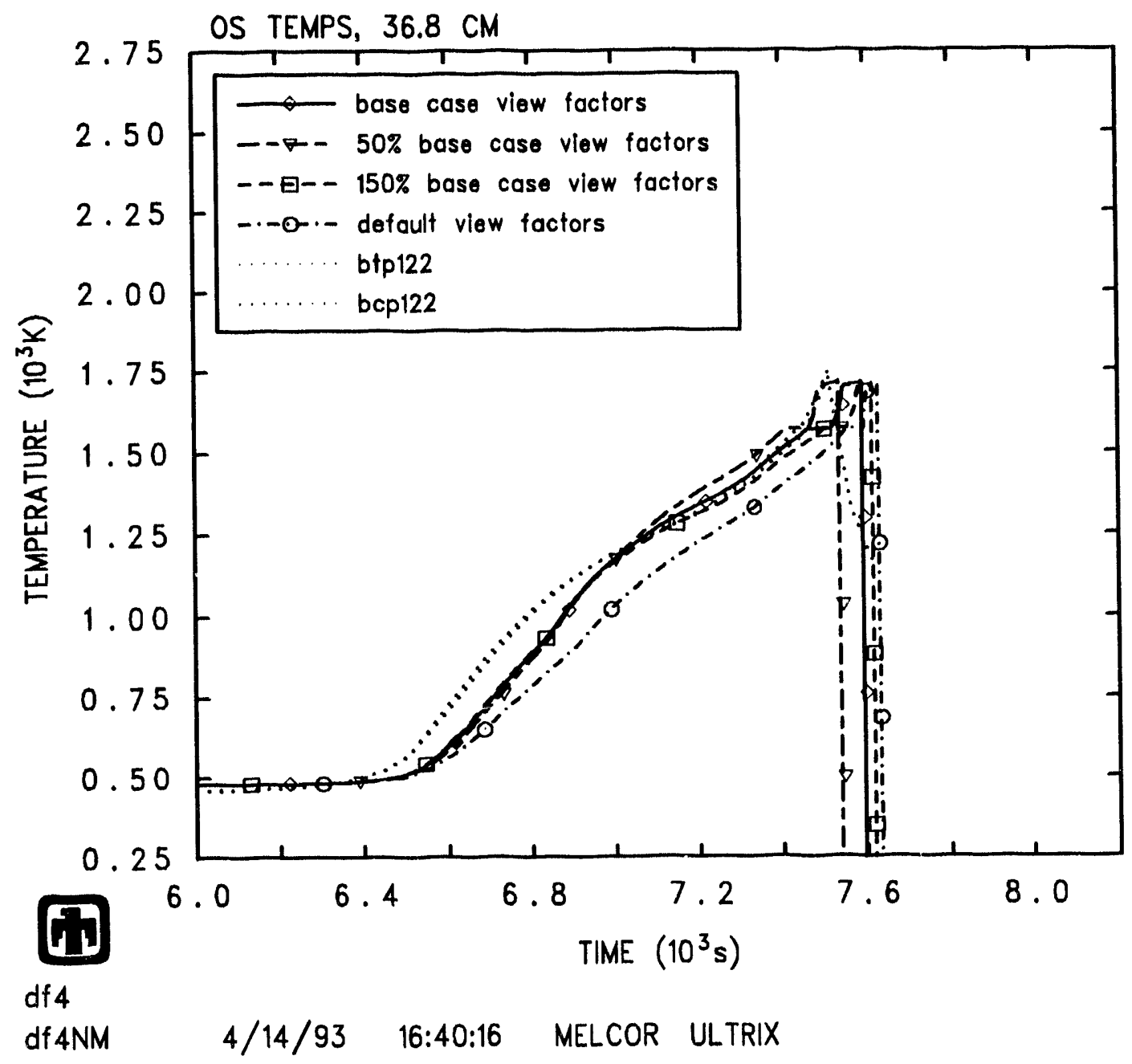

Figure 5.1.7. Control blade temperatures at the $36.8 \mathrm{~cm}$ plane for the radiation view factor sensitivity study, compared to base case and measured values. 


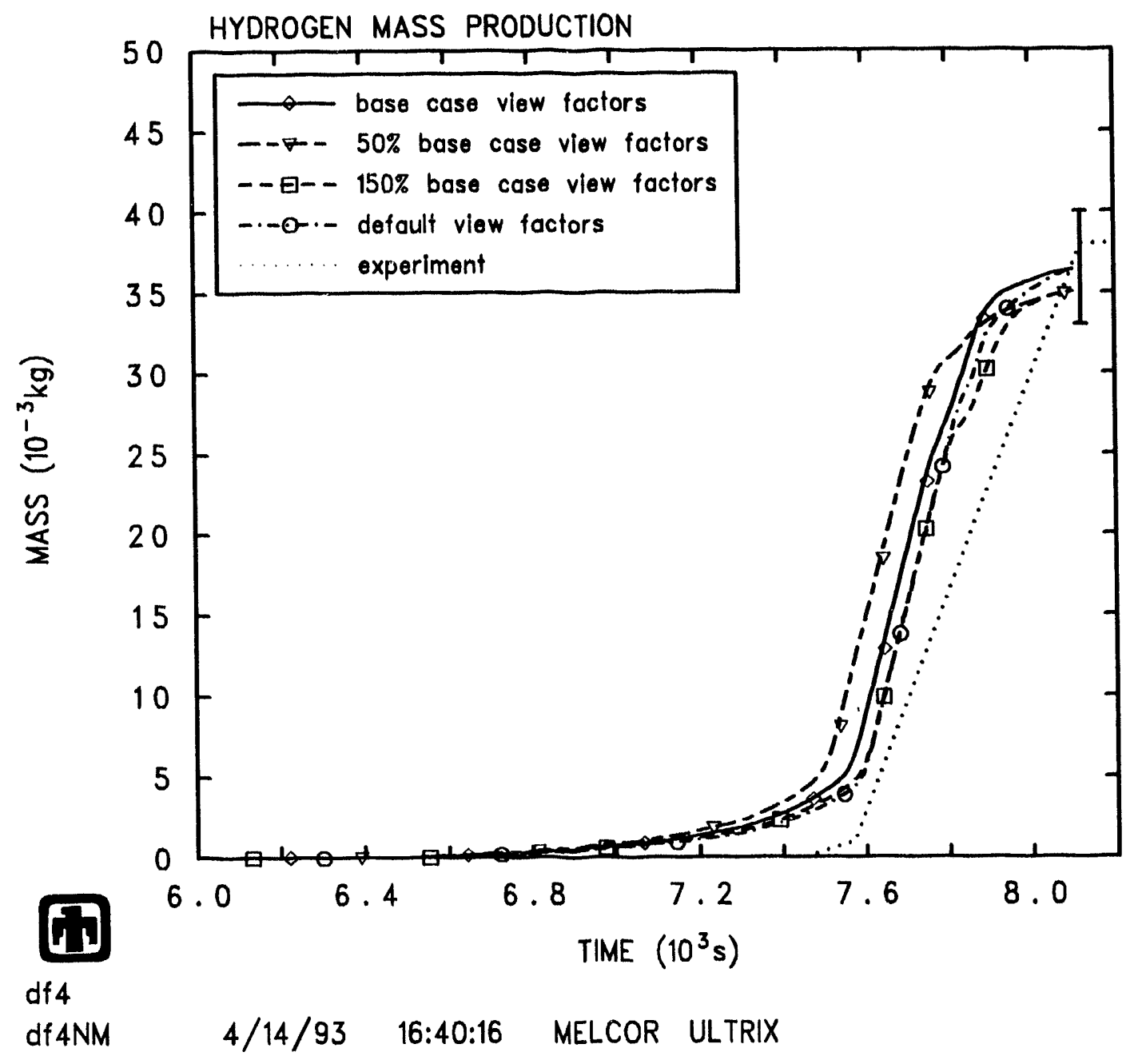

Figure 5.1.8. Hydrogen production for the radiation view factor sensitivity study, compared to base case and measured values. 
temperature and failure timing of the cladding and control blade components showed some sensitivity to where the canister mass was allocated. We suggest allocating canister mass in a manner which best represents the experimental or plant geometry.

\subsubsection{Minimum Component Mass}

The COR package allows the user to set the minimum significant mass for any component in sensitivity coefficient array 1502 . When a component falls below this value its mass and energy are discarded. SC1502 also contains an element for the minimum component mass which is used for time step control. The default values for these coefficients are $10^{-3} \mathrm{~kg}$ and $10.0 \mathrm{~kg}$, respectively (these values are based on typical PWR and BWR cores). In the MELCOR base case model, these limits were set to $10^{-5}$ and $10^{-4} \mathrm{~kg}$, respectively. These are compared with the minimum single-cell initial component mass in the DF-4 model of 4.4 grams, for the control rod poison $\left(\mathrm{B}_{4} \mathrm{C}\right)$ component. In this sensitivity study, the minimum component mass and the minimum mass for temperature control sensitivity coefficients were changed to the default MELCOR values.

The hydrogen production for this case is compared with the base case and measured results in Figure 5.1.12. There was a slight increase in hydrogen production at the end of the problem when the minimum component mass parameters were set to their default values. The temperature results for the cladding and control blade were almost identical to the base case results. The minimum component mass parameters in the COR package did not affect the COR package or the system time step.

Although in this case there were no obvious effects, we nevertheless recommend that the values of these parameters reflect the component masses in the input problem.

\subsubsection{Candling Heat Transfer Coefficient}

The COR package in MELCOR treats candling using an effective heat transfer coefficient between the candling and substrate materials. 'This coefficient is supposed to account for the fraction of component area that is actually covered by the candling material. In this way, the COR package can model either "rivulet" or "film" candling. The default candling heat transfer coefficients were not chosen to model either of these options, but were based on other considerations [17].

The discussion of relocation behavior in Section 4.2 indicates that control blade materials in the MELCOR base case calculation candled a shorter distance before refreezing than was indicated by experimental data. In this sensitivity study, the candling heat transfer coefficients for all materials were reduced to $10 \%$ and $1 \%$ of the default values for all materials in an attempt to more closely match experimental relocation behavior (the default value for candling heat transfer coefficients in MELCOR is $1000 \mathrm{~W} / \mathrm{m}^{2} \mathrm{~K}$ ). These values are input on record COR.00005.

The largest change as a result of reducing the candling heat transfer coefficients in the COR package was expected in the control blade temperatures in the lower half of the test bundle, where most of the candling took place. The cladding and control blade temperatures at that plane are compared to base case and measured results 


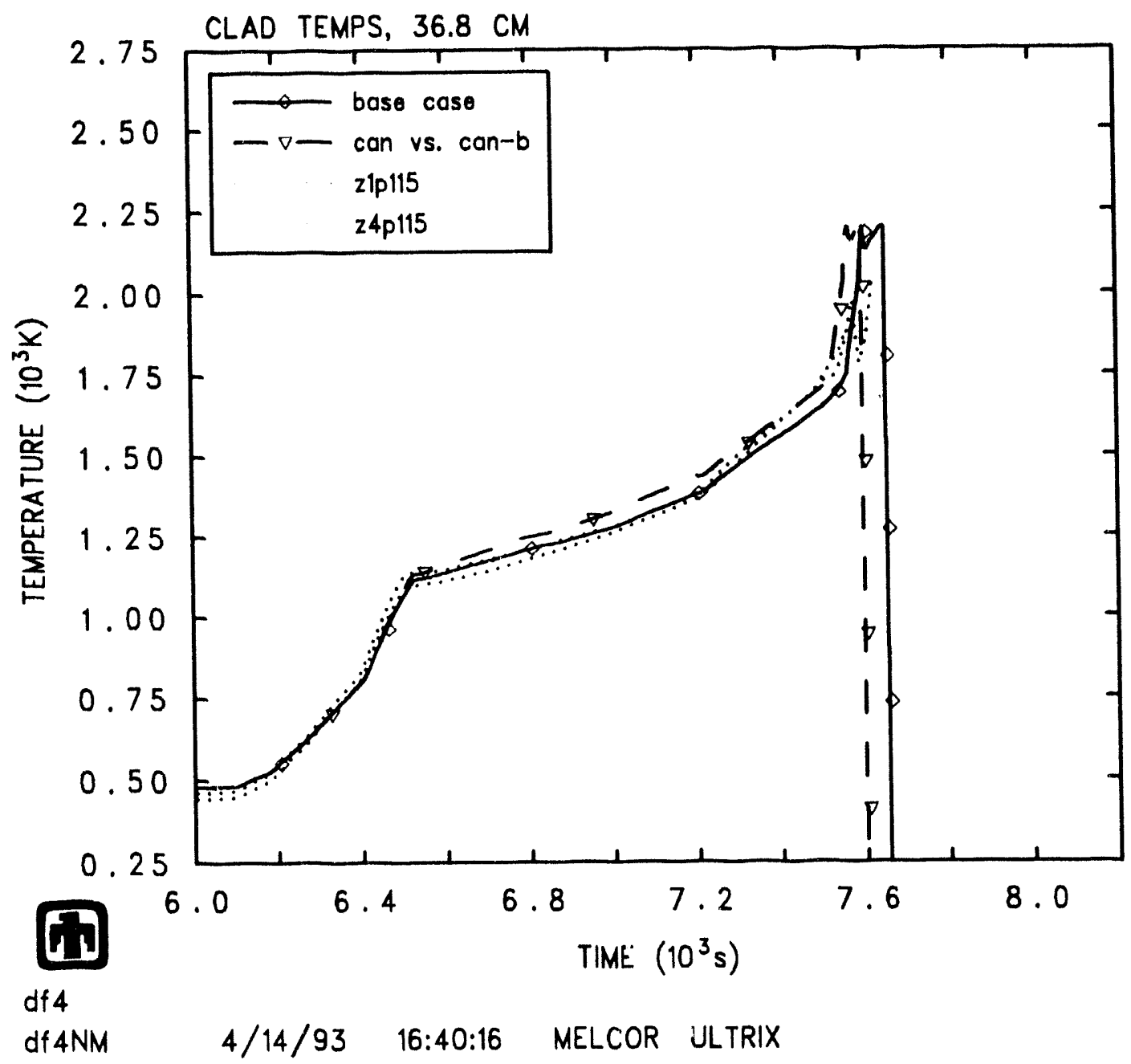

Figure 5.1.9. Cladding temperature response at the $36.8 \mathrm{~cm}$ plane for the canister mass and area partition sensitivity study calculation, compared to base case and measured values. 


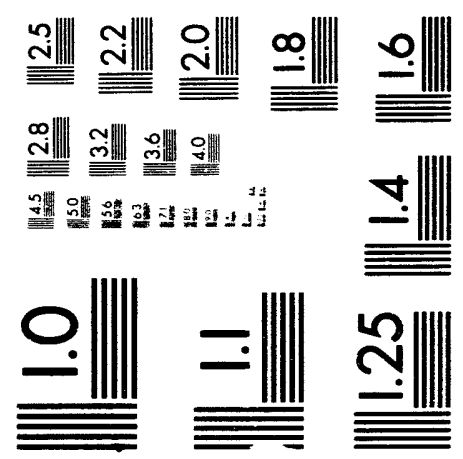



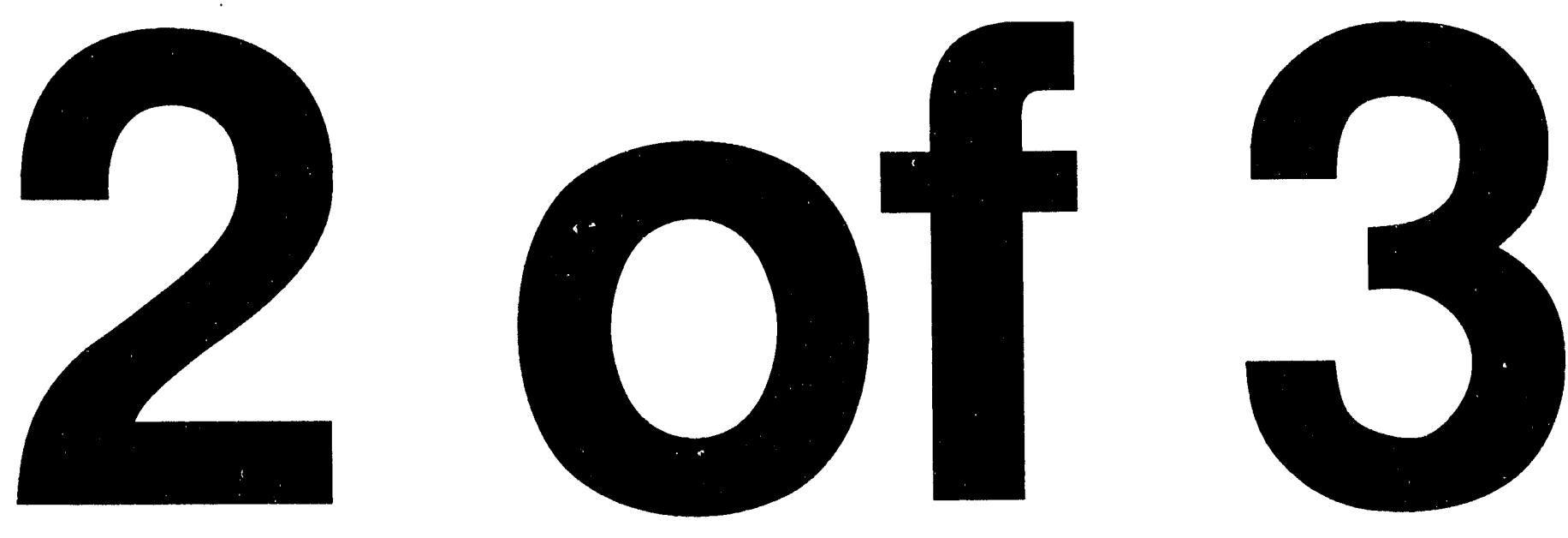


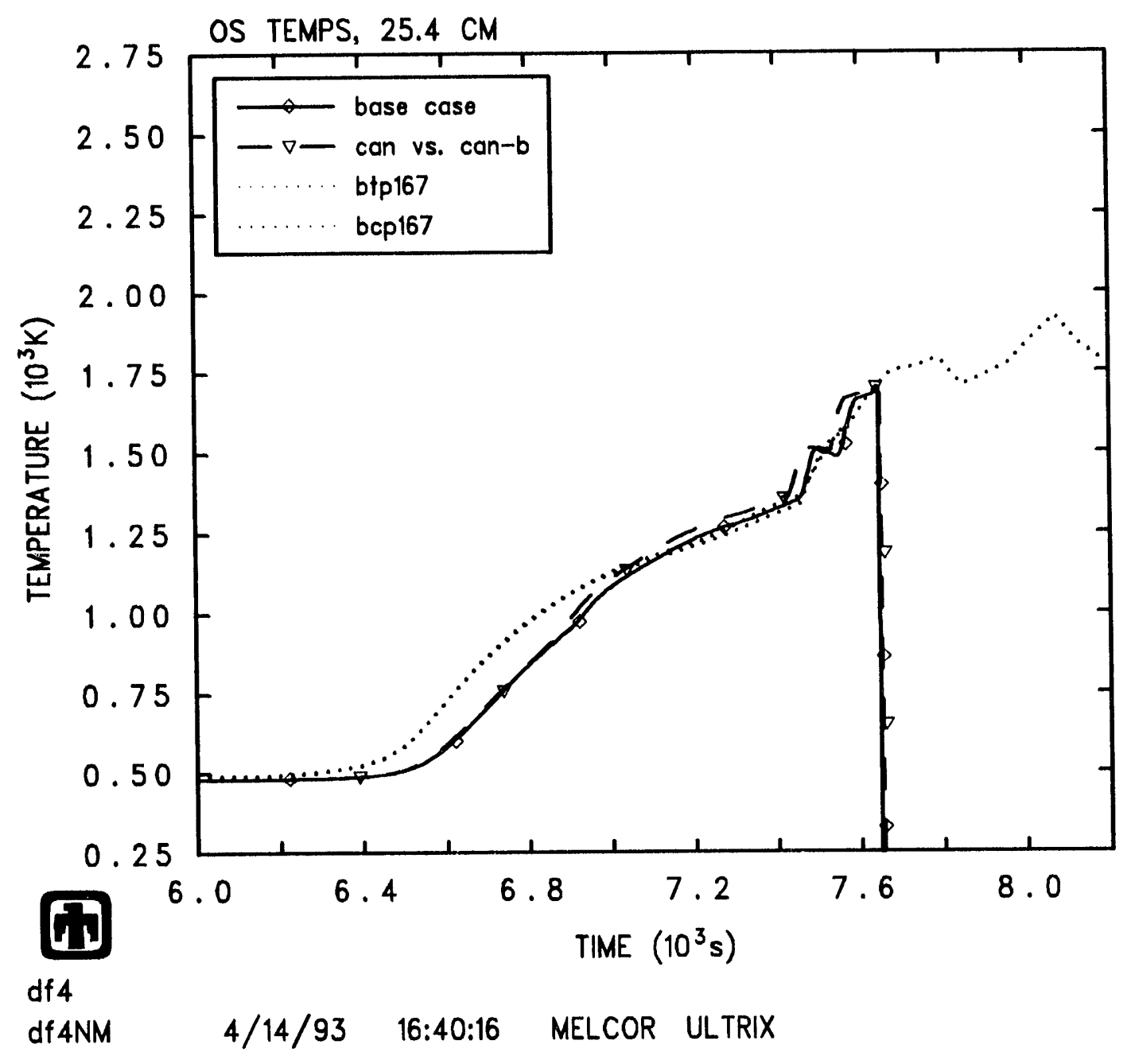

Figure 5.1.10. Control blade temperature response at the $25.4 \mathrm{~cm}$ plane for the canister mass and area partition sensitivity study calculation, compared to base case and measured values. 


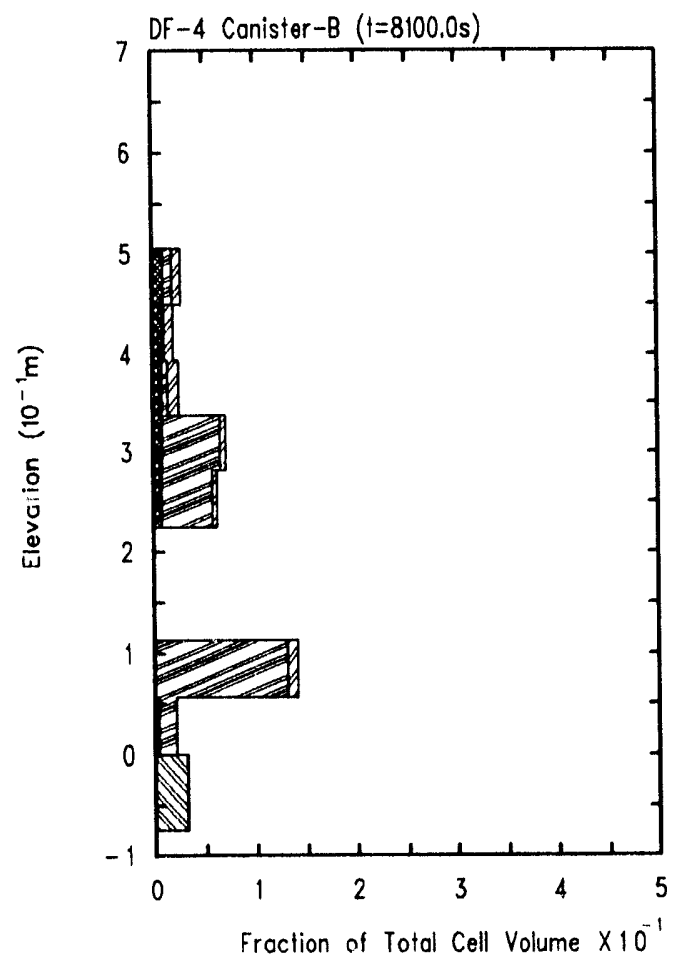

Intact:

$\mathrm{UO}_{2}$

$\mathrm{Zr}$

SS

$\mathrm{ZrO}_{2}$

Stsox

CRP

望 INC

Cong Debris:

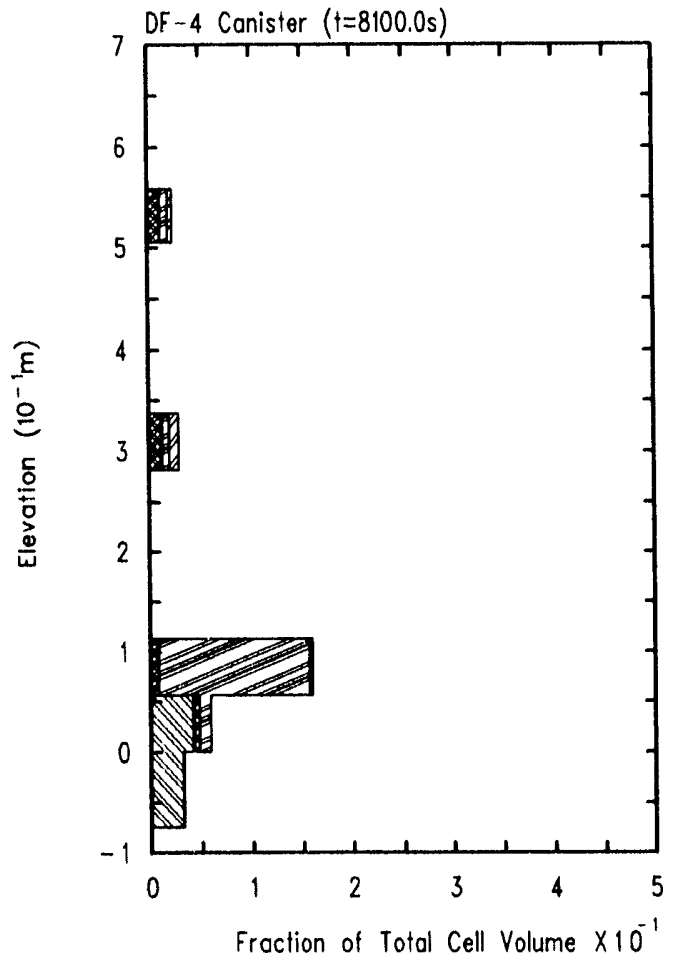

$\mathrm{UO}_{2}$ in Deb

Zr in Deb

SS in Deb

Z $\mathrm{ZrO}_{2}$ in Deb

WSsox in Deb

A CRP in Deb

VIINC in Deb

Figure 5.1.11. Canister component (top) and canister-b component (bottom) material distributions at 8100 seconds for the canister mass and area partition sensitivity study calculation (base case canister-b component material distributions are shown in Figure 4.2.10). 


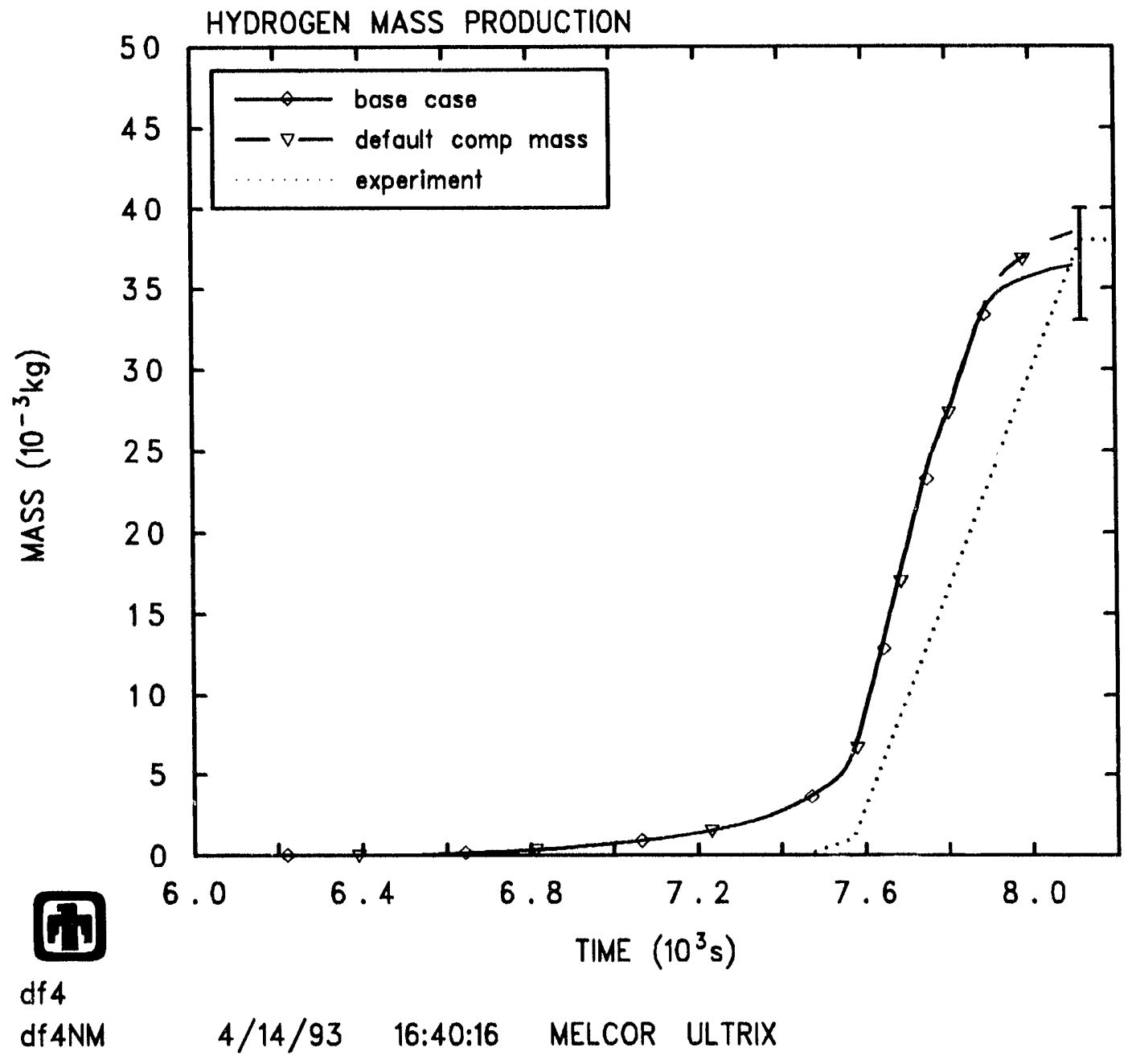

Figure 5.1.12. Hydrogen production for the minimum component mass sensitivity study, compared to base case and measured values. 
in Figures 5.1.13 and 5.1.14, respectively. For the cladding, the early temperature rise due to the arrival of melt was slightly greater for the $1 \%$ case, but otherwise the temperatures were very close. This is not surprising, given that most of the material relocating into this region was solid debris that did not candle.

The results for the control blade were much different. Candling played a significant role in the relocation of control blade material. Decreasing the candling heat transfer coefficient resulted in much better agreement with experimental results in the timing of melt arrival at the base of the control blade. The blockage formed by core materials prevented the control blade at this plane from failing, and allowed this component to heat up beyond the stainless steel melting temperature.

The cladding and control blade temperatures at the $25.4 \mathrm{~cm}$ plane are shown in Figures 5.1.15 and 5.1.16, respectively. The cladding heatup at about 7600 seconds was slightly delayed for the $1 \%$ case. In the base case model a great deal of material refroze at this plane. Reducing the candling heat transfer coefficient forced this material to candle past the $25.4 \mathrm{~cm}$ plane, resulting in less heatup. This effect is more obvious in the control blade, where candling played a stronger role in relocation. The heatup due to melt arrival was delayed slightly for the $10 \%$ case and more significantly for the $1 \%$ case. Note that failure of the control blade at this plane was not delayed by more than 20 seconds even for the $1 \%$ case.

At the $36.8 \mathrm{~cm}$ and $49.5 \mathrm{~cm}$ planes, the temperature behavior was almost identical for all cases, because very little material candled and refroze in these planes.

The hydrogen production for the $10 \%$ and $1 \%$ cases is compared to base case and measured results in Figure 5.1.17. Decreasing the candling heat transfer coefficient slightly decreased the overall hydrogen production. This was the result of refreezing much lower in the test bundle, which decreased the amount of zircaloy heated to autocatalytic oxidation temperatures in the active regions of the core.

The material locations at 7600 seconds for the $1 \%$ case are shown in Figure 5.1.18. This figure can be compared to the base case values at 7600 seconds, shown in Figure 4.2.5. Figure 5.1.18 shows much more control blade (other structure) material at the bottom of the test bundle. This is in better agreement with the PIE and the video record of DF-4, which showed the control blade material to candle much farther down in the test bundle. The fuel and cladding component plot shows that axial level 11 (at $40 \mathrm{~cm}$ ) had not failed at 7600 seconds; this agrees with the slightly delayed cladding temperature shown in Figure 5.1.15.

In conclusion, reducing the candling heat transfer coefficient improved agreement with measured response in the timing of melt arrival at the base of the bundle and in the final distribution of control blade materials. However, it also made the temperature response in the $25.4 \mathrm{~cm}$ plane a bit worse compared to the measured response. The MELCOR user is advised to study the effect of varying the candling heat transfer coefficients in the COR package.

\subsubsection{Minimum Oxide Shell Thickness}

The relocation of core materials after they become molten is controlled by parameters in three MELCOR input records. The minimum oxide shell thickness required 


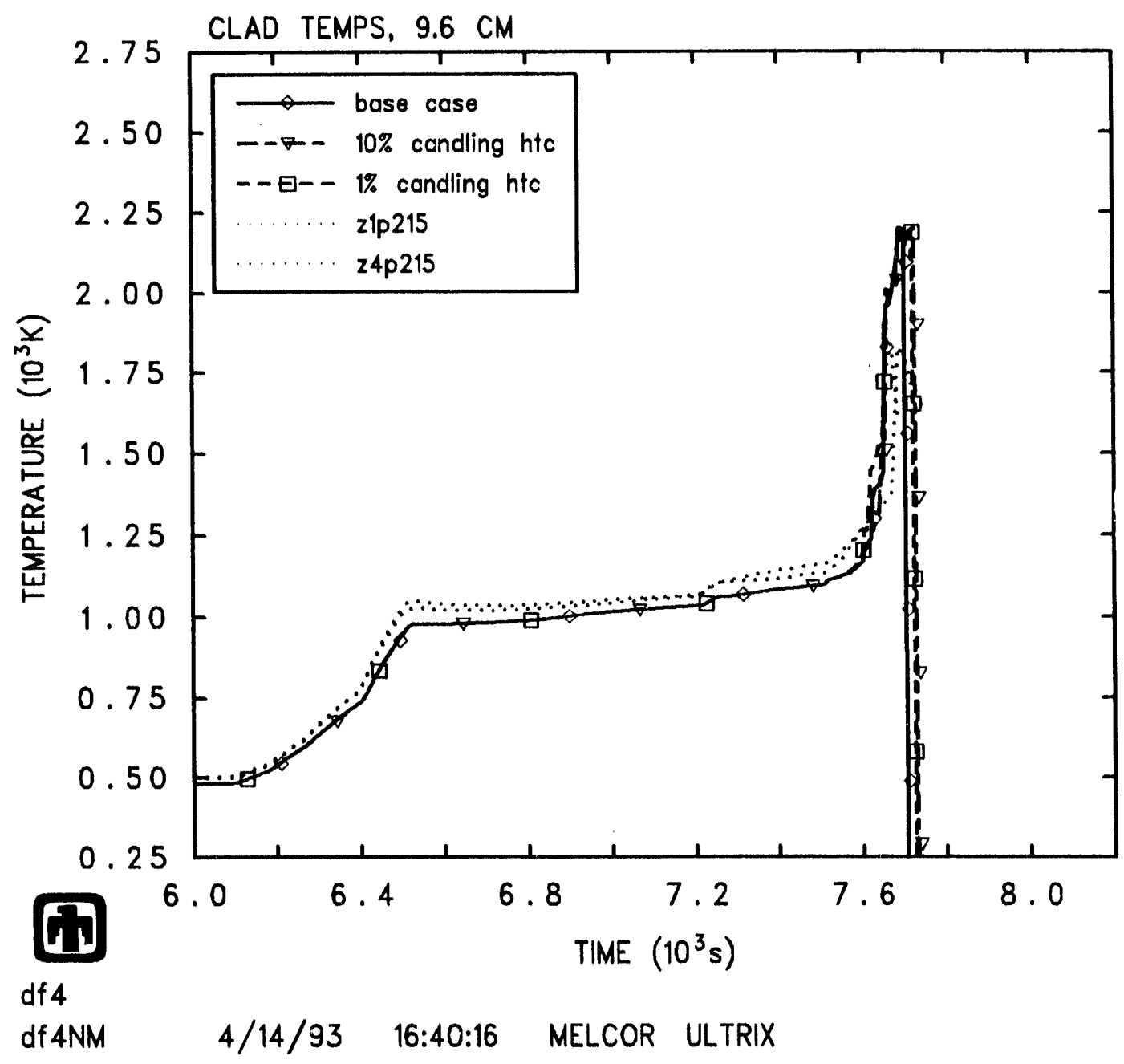

Figure 5.1.13. Cladding temperature response at the $9.6 \mathrm{~cm}$ plane for the candling heat transfer coefficient sensitivity study, compared to base case and measured values. 


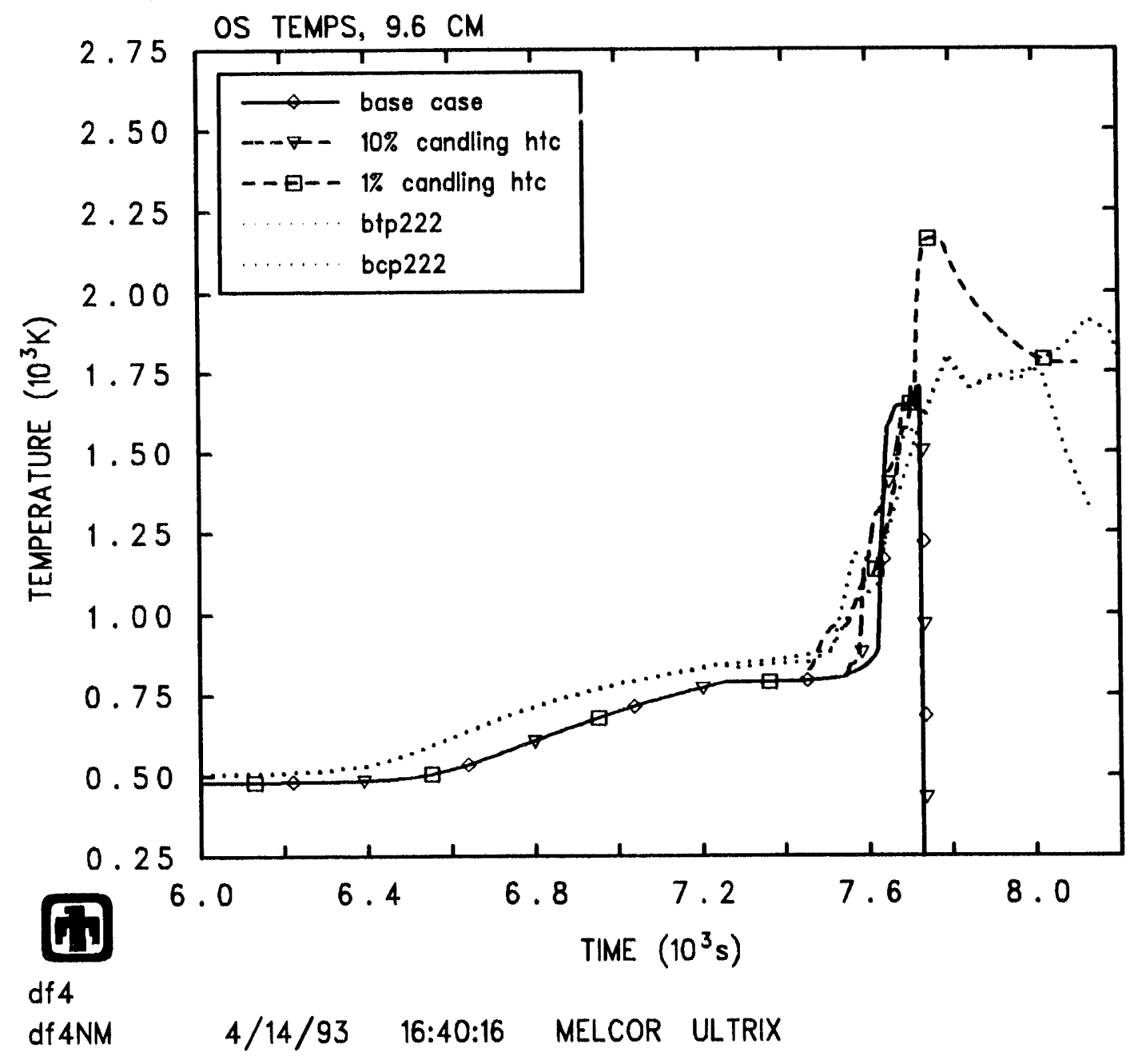

Figure 5.1.14. Control blade temperature response at the $9.6 \mathrm{~cm}$ plane for the candling heat transfer coefficient sensitivity study, compared to base case and measured values. 


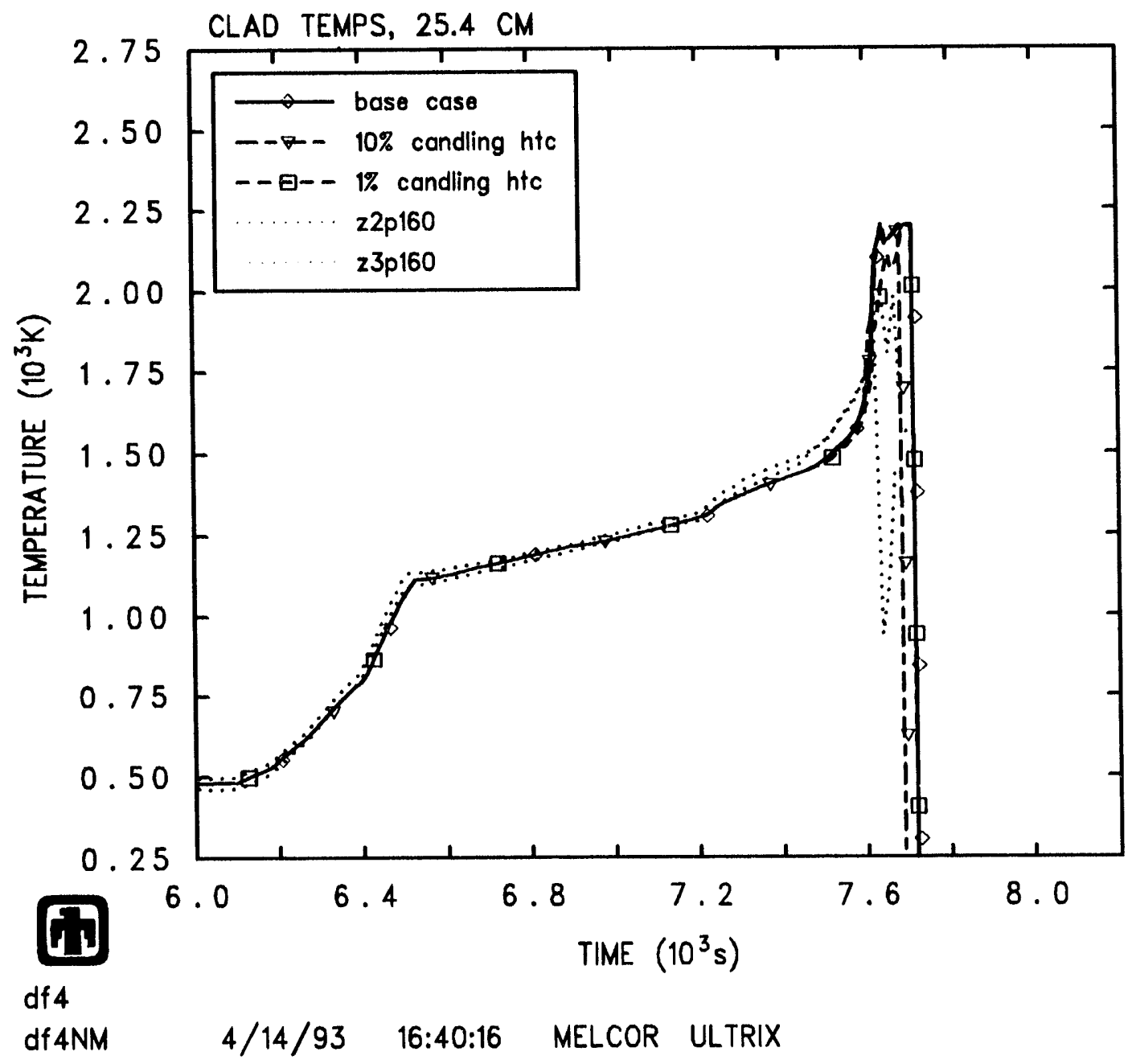

Figure 5.1.15. Cladding temperature response at the $25.4 \mathrm{~cm}$ plane for the candling heat transfer coefficient sensitivity study, compared to base case and measured values. 


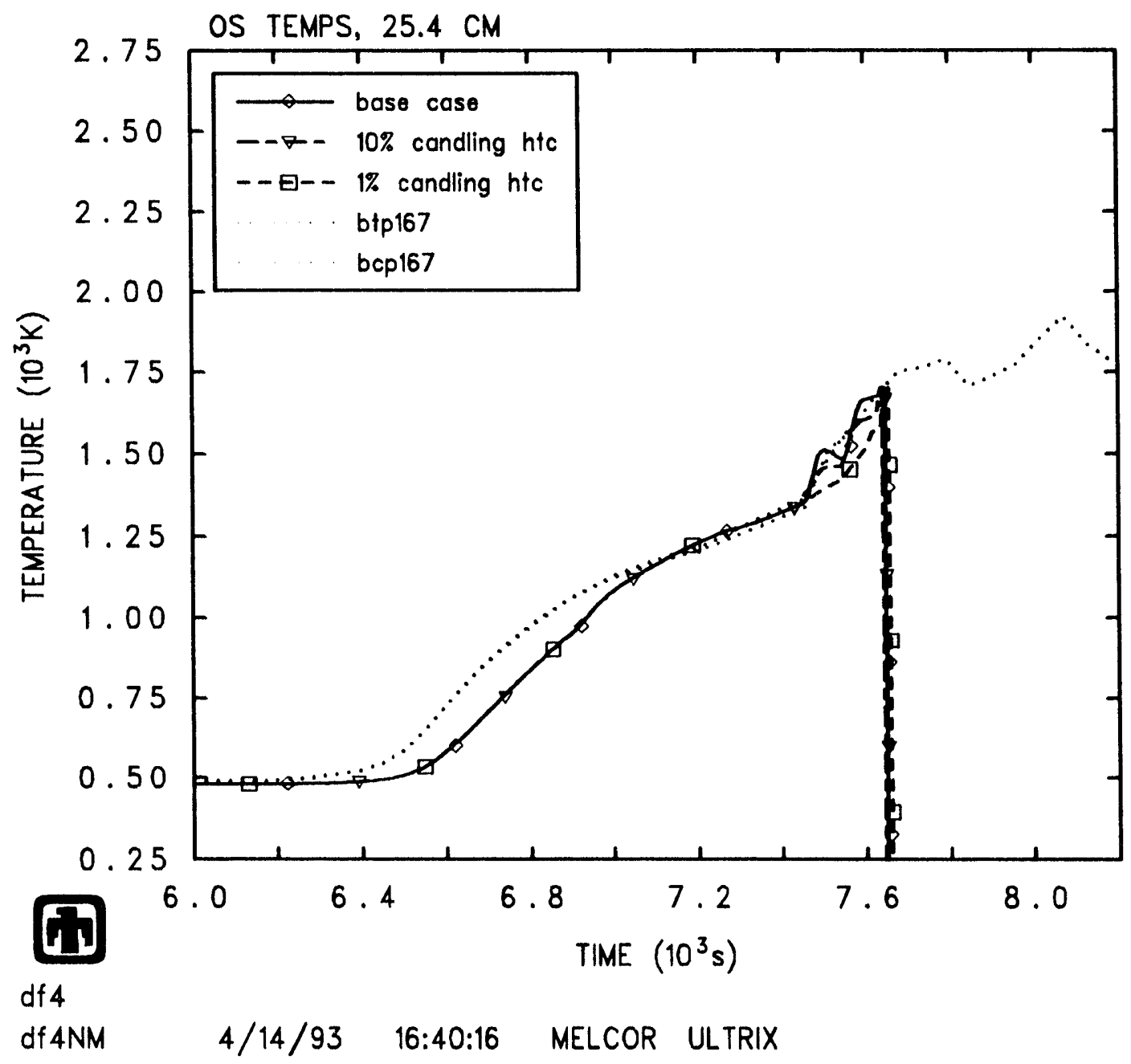

Figure 5.1.16. Control blade temperature response at the $25.4 \mathrm{~cm}$ plane for the candling heat transfer coefficient sensitivity study, compared to base case and measured values. 


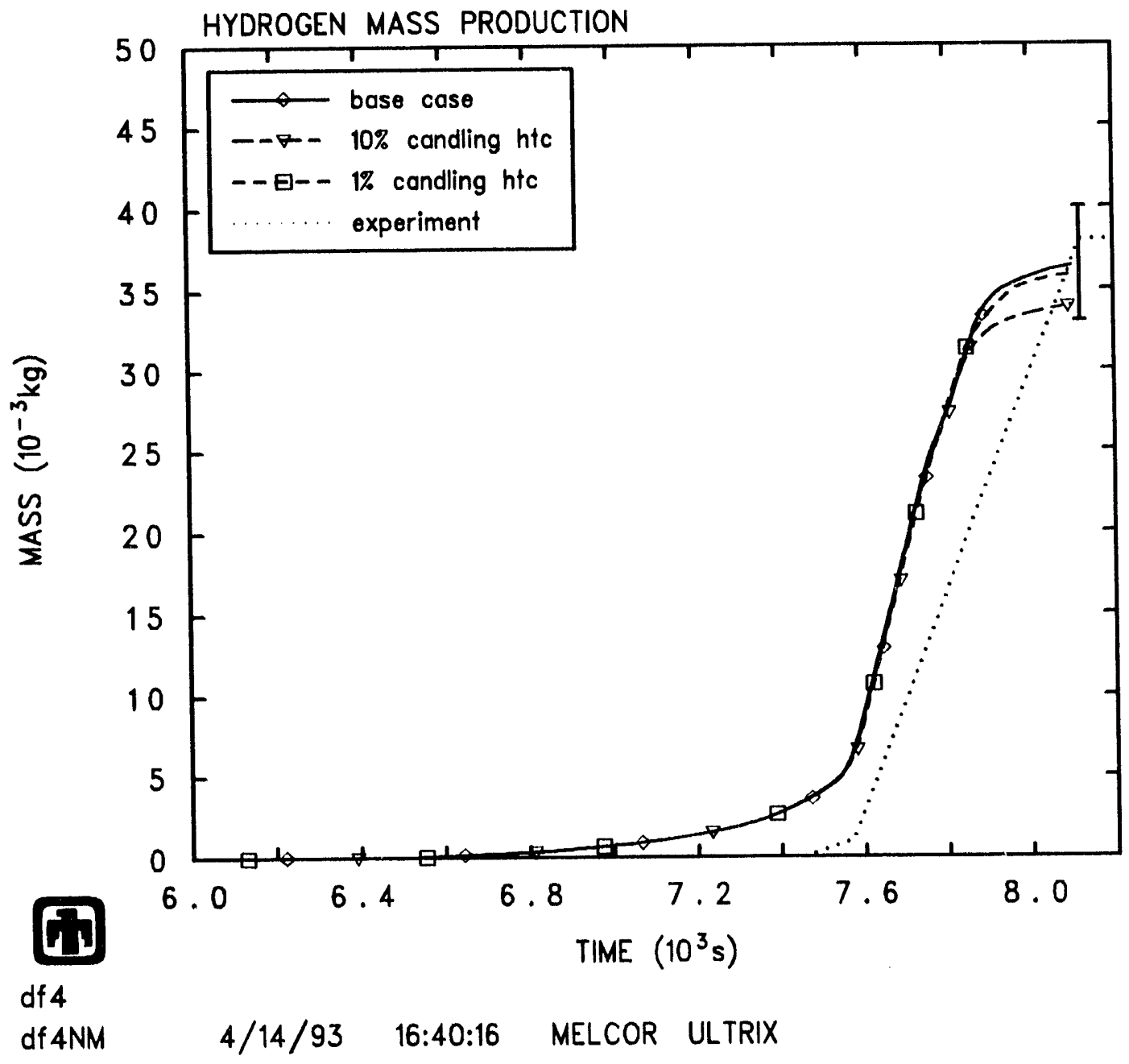

Figure 5.1.17. Hydrogen production for the candling heat transfer coefficient sensitivity study, compared to base case and measured values. 

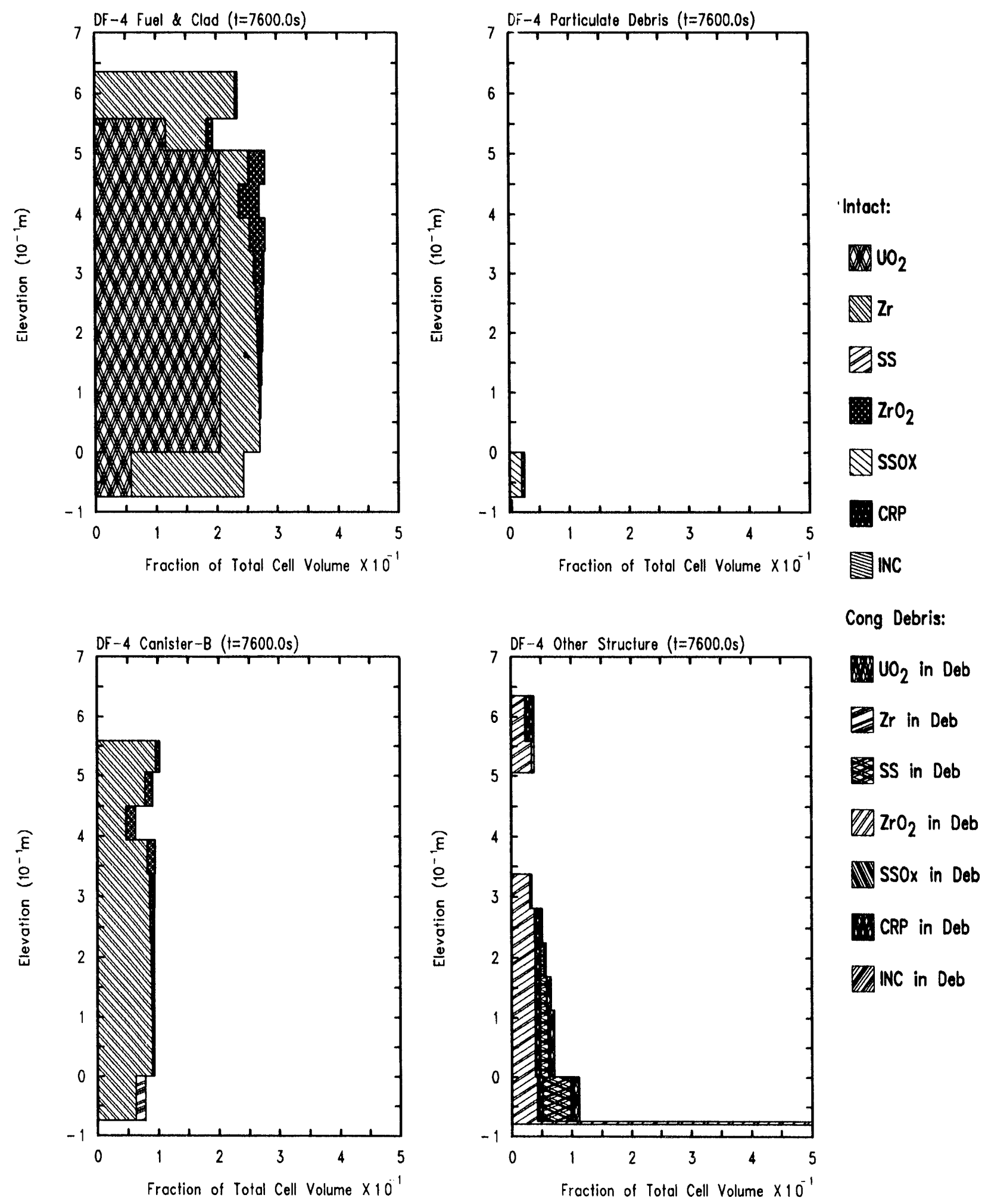

Figure 5.1.18. Component material distributions at 7600 seconds for the $1 \%$ case of the candling heat transfer coefficient sensitivity study. 
to hold up molten material for both zirconium oxide and stainless steel oxide is controlled using sensitivity coefficient array 1131. When materials melt and candle, they are allowed to take along other materials in the same core cell. This is called "secondary material transport," and is intended to model the dissolution of intact material by the candling material (this model is disabled when the eutectics model is active). The transport of secondary materials with the primary candling materials is controlled by parameters input on record COR00007. Finally, the critical minimum thickness of unoxidized zircaloy in the cladding and canister components and of unoxidized stainless steel in the other structure component are input on record COR00008; the zircaloy parameter determines the minimum thickness of unoxidized zircaloy necessary to hold up fuel and cladding materials, while the stainless steel parameter determines the minimum stainless steel thickness required to hold up other structure materials.

In the DF-4 base case input model, the minimum oxide shell thickness needed to hold up molten material at that level was specified to be approximately one tenth the thickness of the oxidizing component. For zircaloy, this parameter was specined as $61 \mu \mathrm{m}$, and for stainless steel it was specified as $142 \mu \mathrm{m}$. The default value for both of these parameters is $1 \mathrm{~mm}$. Also, the default values for the secondary material transport parameters were used. Both critical minimum unoxidized material thicknesses were left at their default values of $0.0001 \mathrm{~m}(0.1 \mathrm{~mm})$.

Two cases were originally designed, each with two changes to the base case. First, the minimum oxide shell thicknesses for both $\mathrm{ZrO}_{2}$ and stainless steel oxide were set to $10 \mu \mathrm{m}$ and $1 \mu \mathrm{m}$. Second, in the same way as was done in the LOFT assessment [11], the transport of secondary materials was disabled in both cases using record COR00007 input. Both of these cases (using $10 \mu \mathrm{m}$ and $1 \mu \mathrm{m}$ for minimum oxide shell thicknesses and no secondary material transport) resulted in almost no difference in temperature behavior throughout the test bundle, for both the cladding and other structure components, and insignificant differences in total hydrogen production.

After further investigation, it was decided to run two more cases for this sensitivity study, each of which had three changes to the base case. The first two changes were identical to those in the previous paragraph. In addition, the debris formation parameters in record COR00008 were adjusted such that no unoxidized zircaloy and stainless steel was required to hold up the associated components. It was later determined that changing the secondary material transport parameters had no effect on the results, since the secondary transport model in MELCOR is disabled when the eutectics model is active.

For the latter two sensitivity study calculations, the changes to component temperatures were also relatively minor. For example, the cladding temperatures at the $36.8 \mathrm{~cm}$ plane are shown in Figure 5.1.19. The only difference with base case temperatures in this case was that the cladding component at the $36.8 \mathrm{~cm}$ plane did not fail. This was due to the oxide layer preventing the conversion of fuel, cladding and oxides at that axial level to debris after all intact zircaloy was depleted. The control blade temperatures at the $36.8 \mathrm{~cm}$ plane are shown in Figure 5.1.20, and the hydrogen production is shown in Figure 5.1.21. There was little change in the control blade temperatures and the overall hydrogen production. 
The final material locations for the $1 \mu \mathrm{m}$ case are shown in Figure 5.1 .22 (the $10 \mu \mathrm{m}$ case was almost identical). This should be compared to Figure 4.2.10 for the base case. The adjustment of debris formation parameters strongiy affected the final material locations, as expected. All of the control blade relocated, due to the dissolution of control blade materials by $\mathrm{B}_{4} \mathrm{C}$, while the fuel component was intact at all levels, held up by a layer of $\mathrm{ZrO}_{2}$.

The fuel pellet stacking that resulted from the adjustment to the parameters described in this section was more realistic when compared to the final debris state in DF-4. However, these adjustments also resulted in the full oxide layer remaining in place of the intact cladding. This is in contrast to DF-4, where much of the oxidized cladding was dissolved by candling zircaloy. When modeling experiments that exhibit fuel pellet stacking, these parameters should probably be adjusted to allow MELCOR to leave fuel pellets stacked.

\subsubsection{Heat Structure Boundary Fluid Temperature Option}

The COR package $\mathrm{d} T / \mathrm{d} z$ model calculates the rise in control volume atmosphere temperature as the atmosphere vapor travels through core cells. 'This model provides a finer resolution of corc atmosphere temperatures than the CVH package is able to provide. These $\mathrm{d} T / \mathrm{d} z$ temperatures are used to calculate heat transfer between the fluid and the core components and radial boundary heat structures. The default temperature used to calculate heat transfer with the radial boundary heat structures is the bulk CVH atmosphere temperature (the control volume bulk pool temperatures are used below the liquid level in each core control volume). For this project, an option was implemented in the HS and COR packages to use local $\mathrm{dT} / \mathrm{d} z$ temperatures instead of bulk CVH atmosphere temperatures to calculate heat transfer to the radial boundary heat structures. This option was implemented in the release version of MFLCOR and was used for all radial boundary heat structures in the DF-4 base case model. In this sensitivity study, the default MELCOR option was specified for all radial boundary heat structures so that CVH bulk atmosphere temperatures were used to calculate heat transfer with radial heat structures. This option is specified on the HSCCCCCO04 record.

The control volume bulk atmosphere temperature for this case is compared with base case results in Figure 5.1.23. Using the default option in all cells had the effect of raising control volume bulk atmosphere temperatures. This was the result of the artificial heatup of the heat structure surfaces in the lower core cells by bulk CVH atmosphere temperatures which were higher than the $\mathrm{d} T / \mathrm{d} z$ temperatures in those lower core colls. The resulting cladding and control blade temperatures at the 36.8 cm plane are shown in Figures 5.1 .24 and 5.1 .25 , respectively. Raising the CVH bulk atmosphere temperature increased the cladding and control blade temperatures, and resulted in earlier component. failure. 'The hydrogen production for both cases 


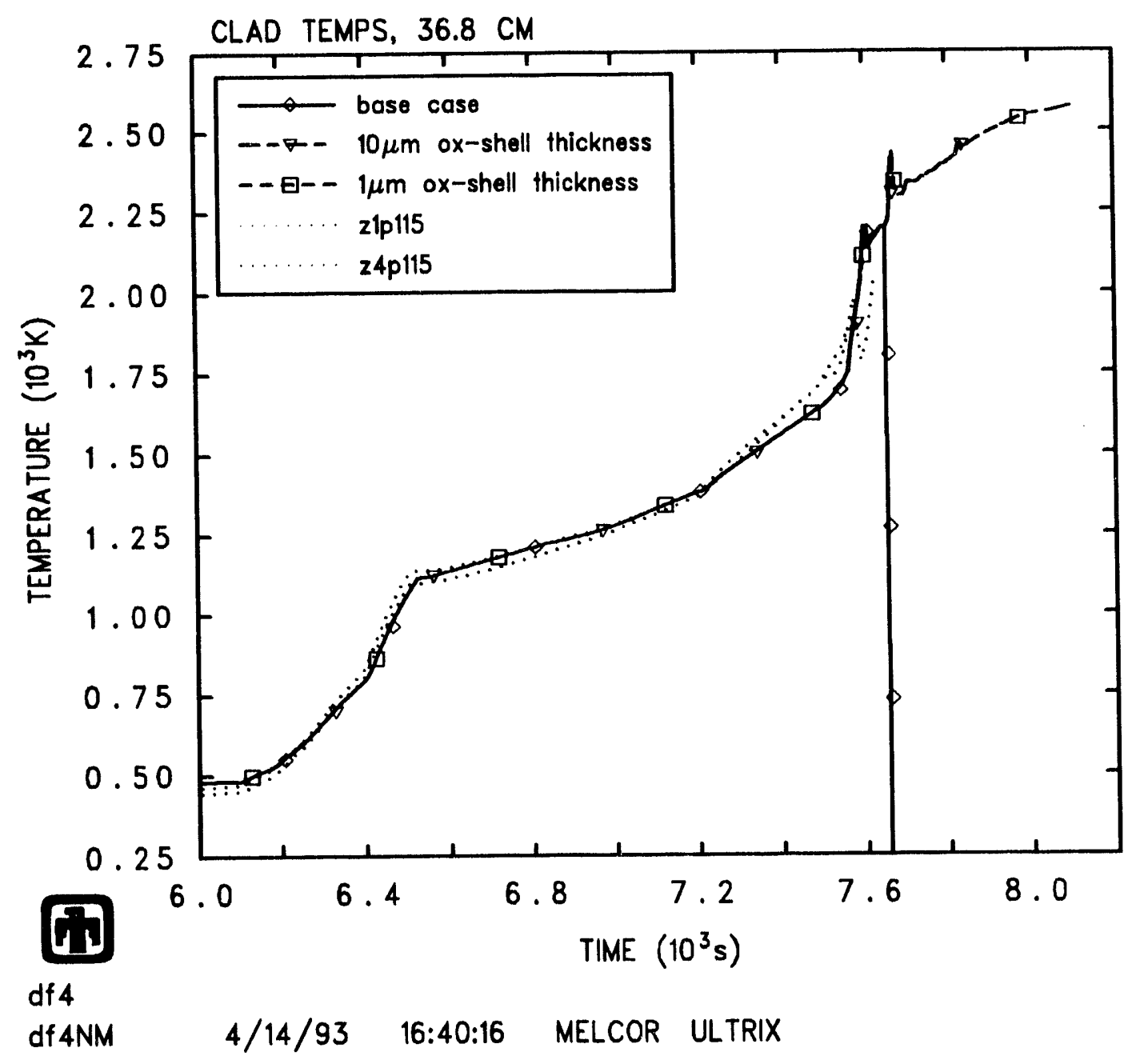

Figure 5.1.19. Cladding temperatures at the $36.8 \mathrm{~cm}$ plane for the minimum oxide shell thickness sensitivity study, compared to base case and measured values. 


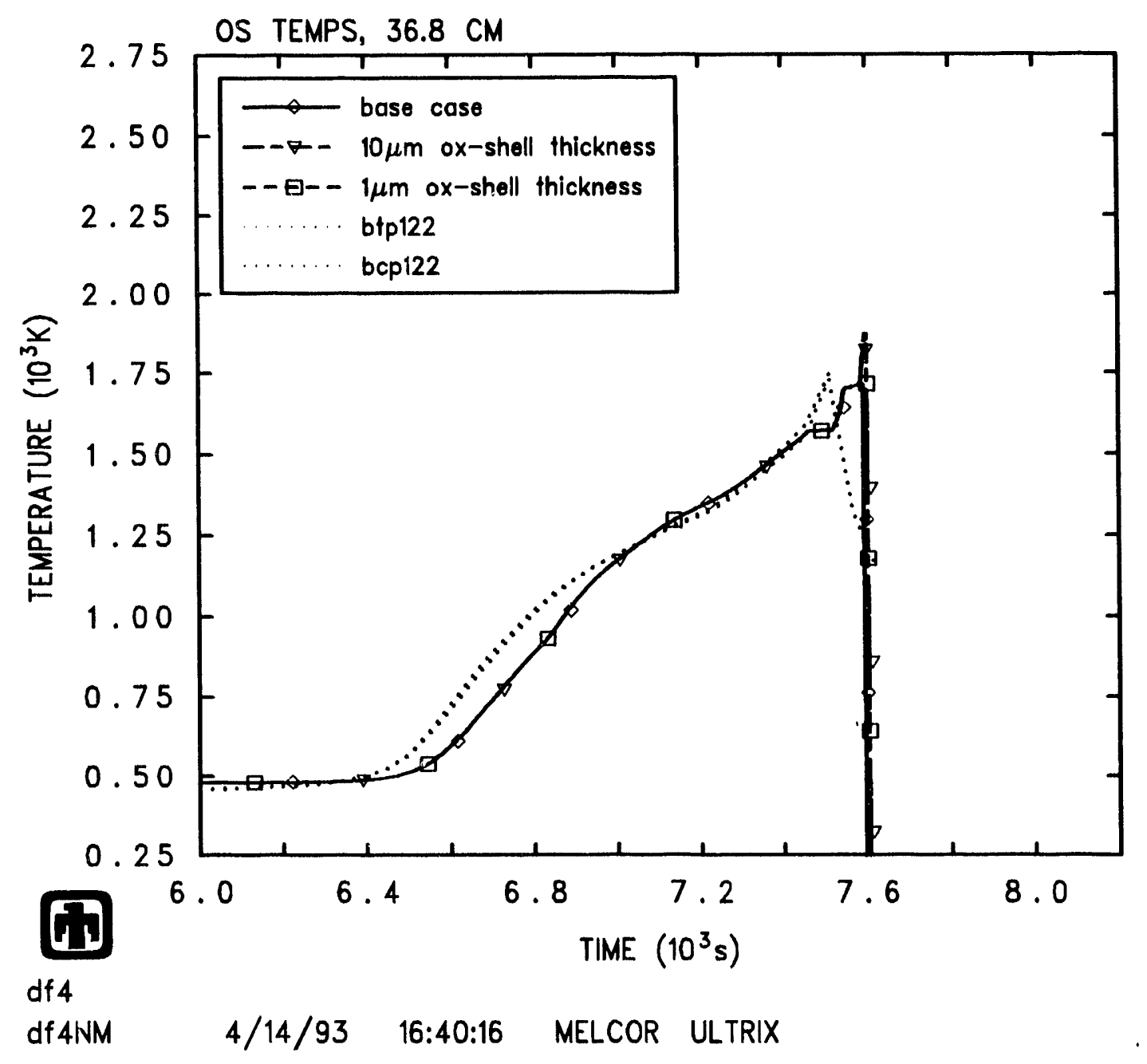

Figure 5.1.20. Control blade temperatures at the $36.8 \mathrm{~cm}$ plane for the minimum oxide shell thickness sensitivity study, compared to base case and measured values. 


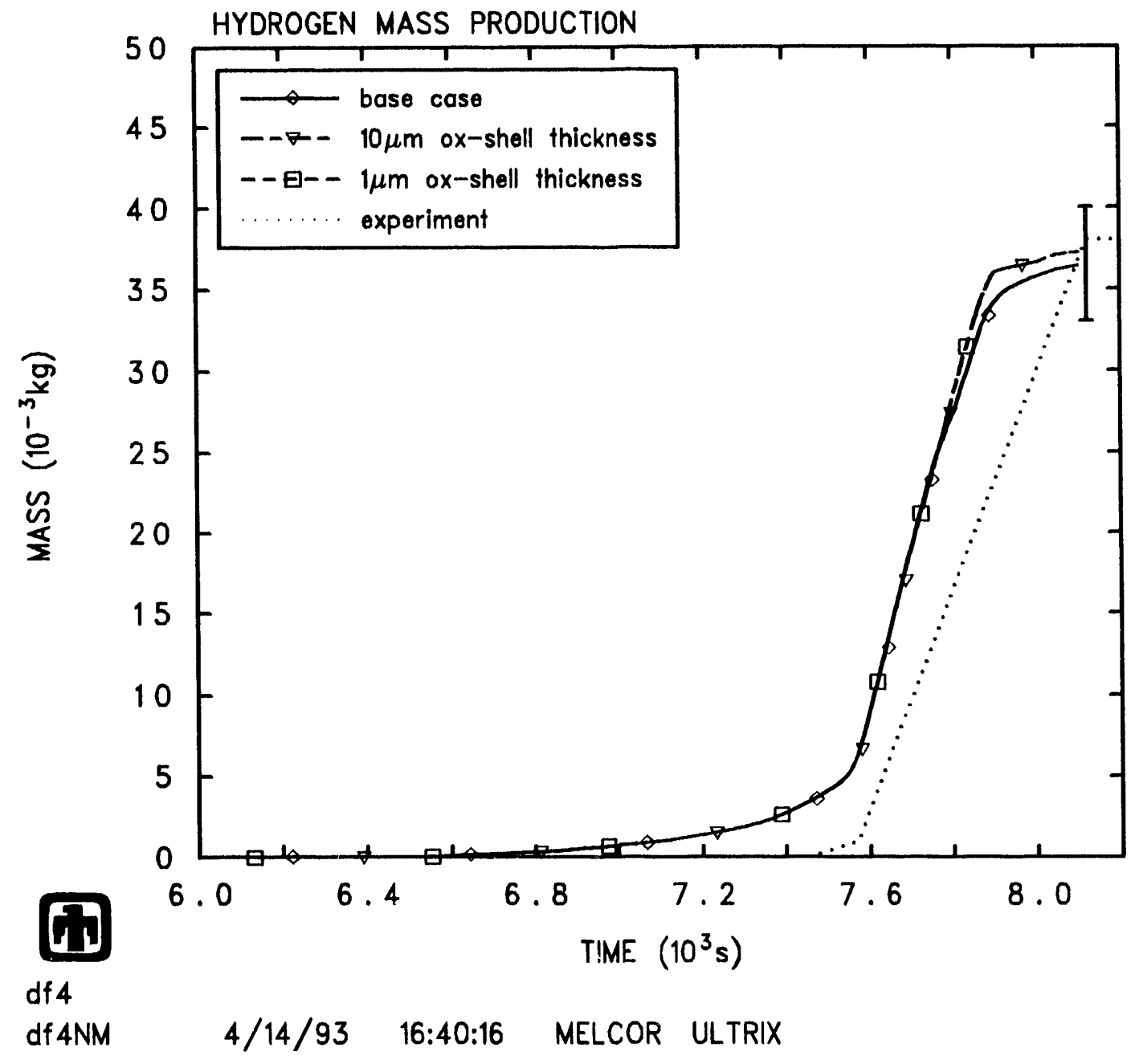

Figure 5.1.21. Hydrogen production for the minimum oxide shell thickness sensitivity study, compared to base case and measured values. 

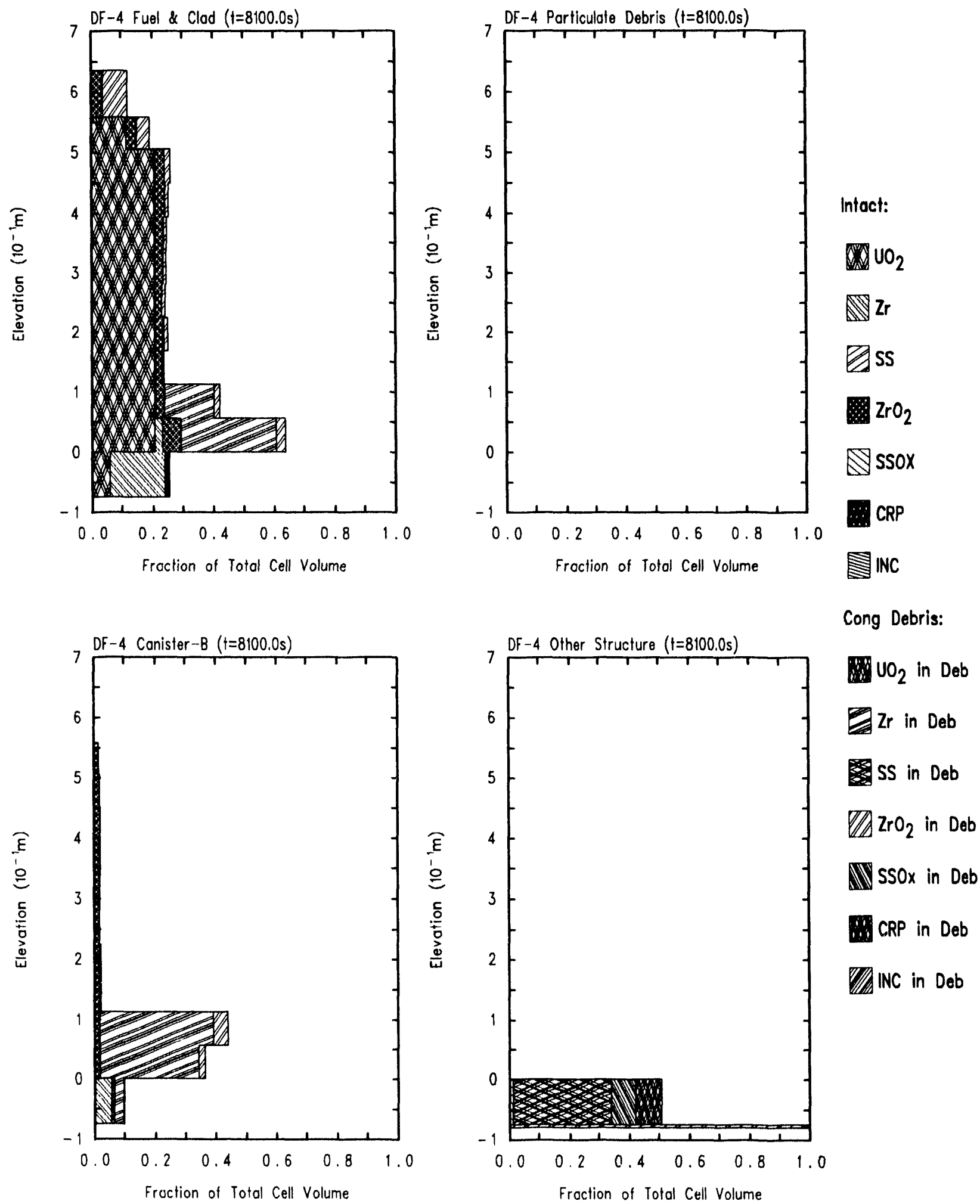

Figure 5.1.22. Component material distributions at 8100 seconds for the $1 \mu \mathrm{m}$ case of the minimum oxide shell thickness sensitivity study. 
is shown in Figure 5.1.26. The early component failure decreased the time available for the oxidation of intact core components, which resulted in less overall hydrogen production. The hydrogen production in the sensitivity study case was moved forward in time as well, due to the increased cladding temperatures.

The effect of the new boundary heat structure fluid temperature option was to lower core component temperatures and delay component failure. Using this option more accurately models the heat transfer with radial boundary heat structures. It is therefore recommended that this new option be used in future MELCOR modeling efforts.

\subsection{Debris Behavior Studies}

Two sensitivity studies were performed to study the effects of varying debris behavior parameters in MELCOR. These studies concerned the debris porosity and the particulate debris diameter specified in COR package input.

\subsubsection{Debris Porosity}

The debris porosity is specified for each axial level in the COR package on record CORZjj01. The intact material porosity is also specified on this record. For this sensitivity study, the debris porosity was increased and decreased from its base case value of 0.50 , resulting in porosities of 0.30 and 0.70 . The intact porosity was also increased from the base case value of 0.0 to 0.25 for both cases. There are no default values for intact or debris porosities. Increasing the porosity of a component had the effect of increasing the volume occupied by that component, and raising the upper surface of the debris pile in the bottom of the test bundle.

Decreasing the debris porosity had a relatively large effect on the amount of hydrogen produced. This was especially true for the DF-4 experiment, where more than $50 \%$ of the hydrogen was produced as a result of debris oxidation. Figure 5.2.1 shows that decreasing the debris porosity decreased the amount of hydrogen produced by about 10 grams, or $26 \%$. Increasing the porosity increased the hydrogen produced by $12 \%$. Changing the debris porosity changed the amount of debris surface area exposed to the control volume atmosphere. It also affected how much cladding was exposed to the atmosphere, since the debris porosity determined the height of debris stacked in the bottom of the test bundle.

The final state of the particulate debris component in the test bundle for the small debris porosity case (porosity $=0.30$ ) is shown in Figure 5.2.2, while the final state of the particulate debris component for the large debris porosity case (porosity $=0.70$ ) is shown in Figure 5.2.3 (these can be compared with the final state of components in the base case, shown in Figure 4.2.10). These figures show that decreasing the debris porosity resulted in more debris at lower axial levels. This increased debris concentration led to earlier cladding failure at those levels (see Figure 5.2.4). The temperature response for the control blade, however, shows that decreasing the debris porosity delayed slightly the arrival of melt at the $9.6 \mathrm{~cm}$ plane (see Figure 5.2.5). 


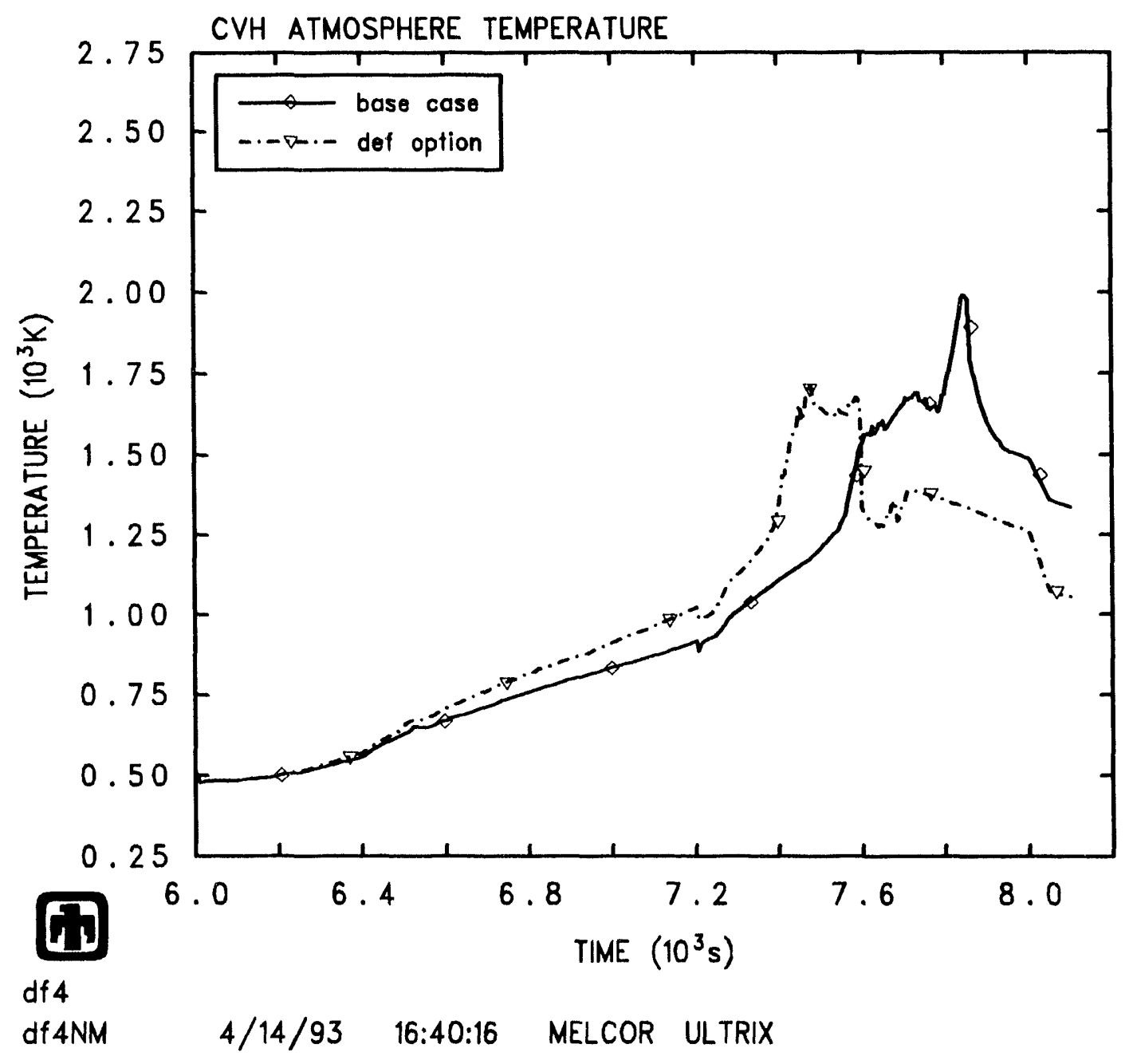

Figure 5.1.23. CVH bulk atmosphere temperature for the default boundary fluid temperature option sensitivity study, compared to base case values. 


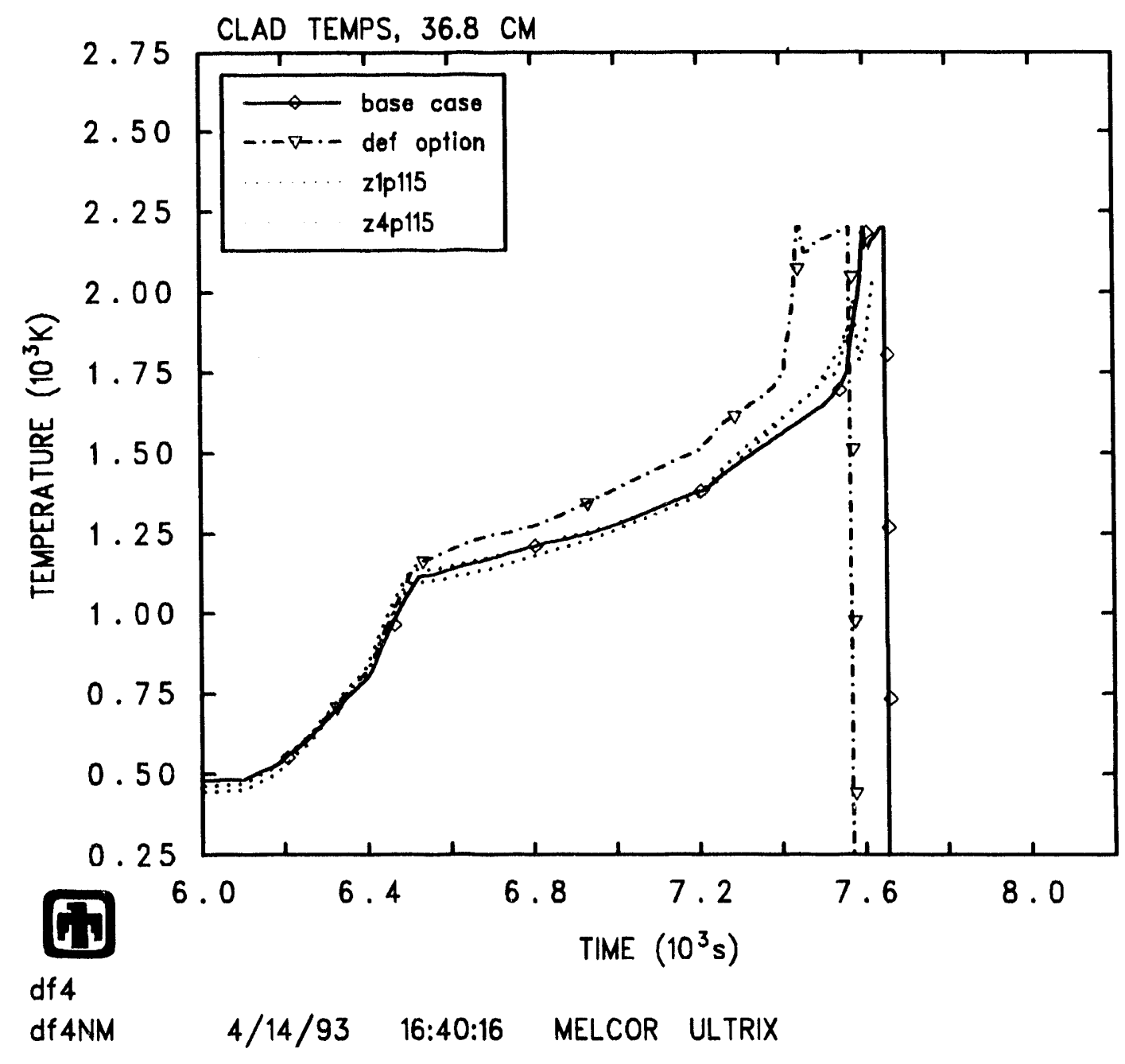

Figure 5.1.24. Cladding temperatures at the $36.8 \mathrm{~cm}$ plane for the default boundary fluid temperature option sensitivity study, compared to base case and measured values. 


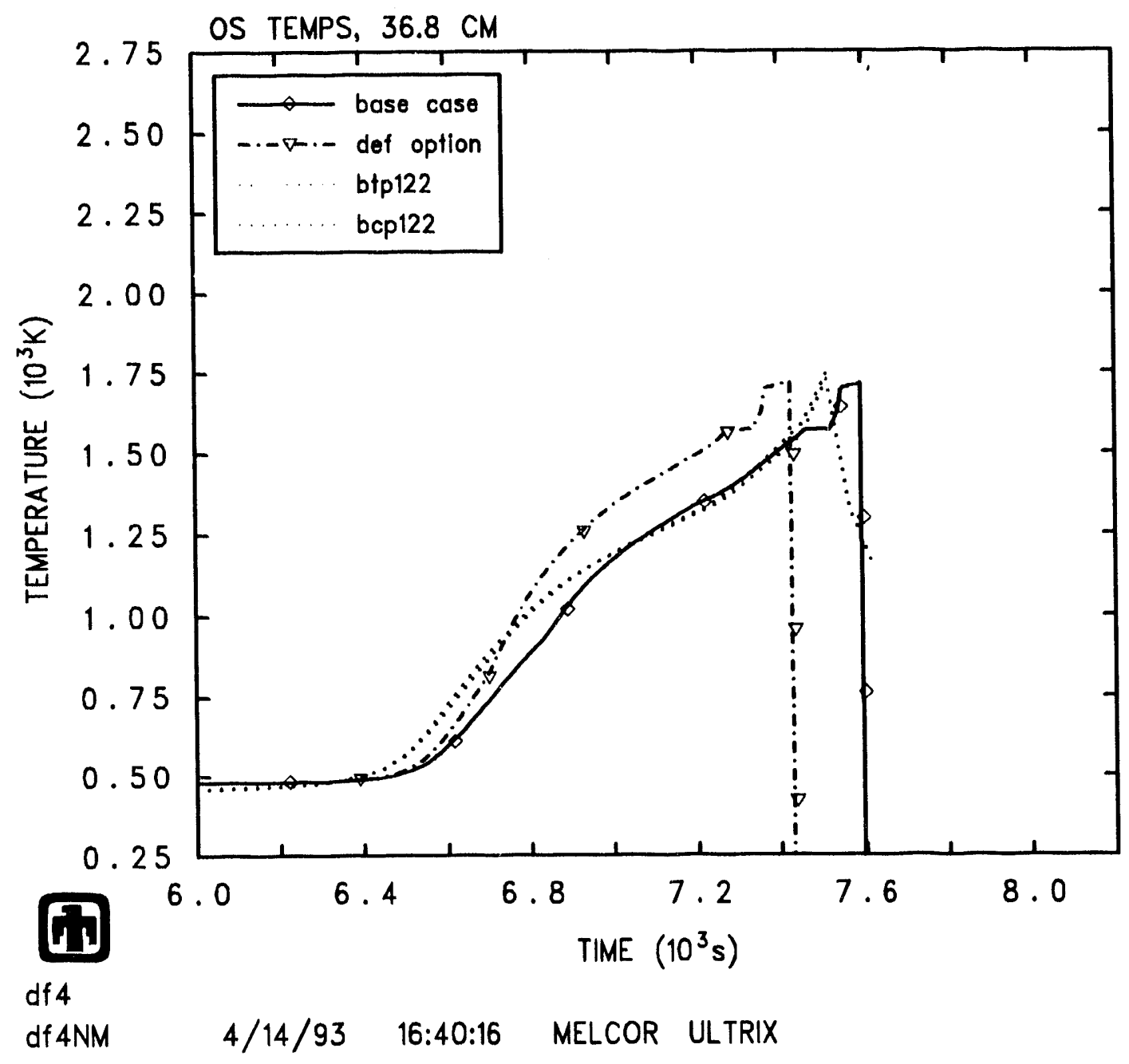

Figure 5.1.25. Control blade temperatures at the $36.8 \mathrm{~cm}$ plane for the default boundary fluid temperature option sensitivity study, compared to base case and measured values. 


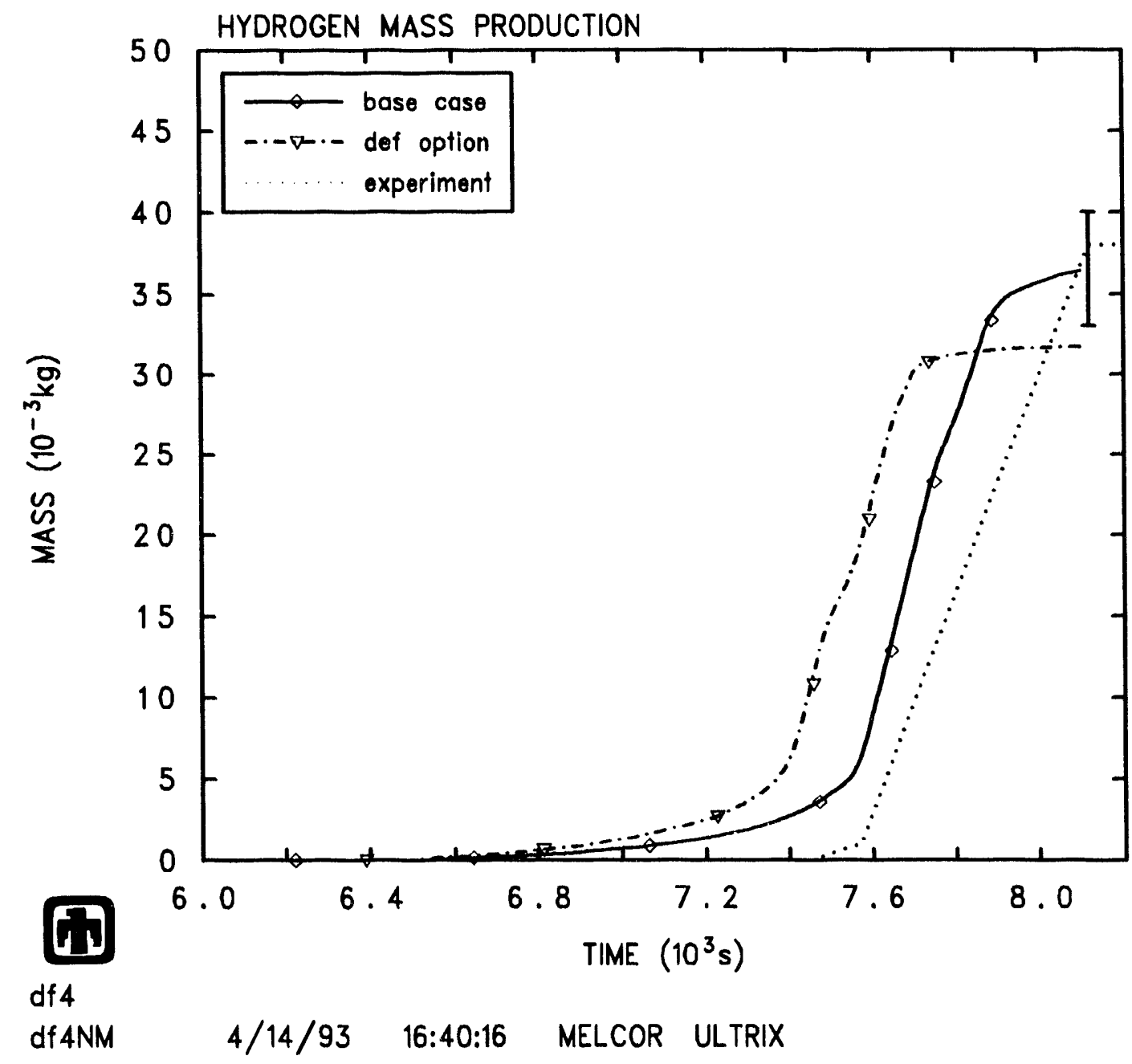

Figure 5.1.26. Hydrogen production for the default boundary fluid temperature option sensitivity study, compared to base case and measured values. 
This was due to the delayed heatup of the control blade further up the bundle, where there was less debris stacked up outside the canister wall.

This study shows that the hydrogen production was sensitive to the debris porosity, and to a lesser extent temperatures in the lower portions of the bundle were affected as well. The volume occupied by debris was also strongly affected. The debris porosity could be more important in problems where the debris resided in the lower plenum. The debris porosity would determine the amount of decay heat emitted by the debris in the lower plenum (because it affects the mass of debris in a given volume) and would affect the debris surface area in contact with the pool. The timing of lower head failure would depend on both of these effects.

\subsubsection{Debris Diameter}

The diameter of particulate debris determines the debris surface areas used for heat transfer and oxidation (this parameter is input on record CORijj04). In the MELCOR base case model for DF-4, the debris diameter was set to $8.26 \mathrm{~mm}$, which was the diameter of fuel pellets in the test bundle. In this sensitivity study, the debris diameter was increased and decreased by a factor of two.

The hydrogen production for the two cases is compared to the base case and measured results in Figure 5.2.6. This shows that reducing the debris diameter resulted in a substantial increase in hydrogen production. This result is not surprising, given that decreasing the debris diameter increased the debris surface area available for oxidation, and a large amount of debris oxidation occurred in DF-4. Increasing the debris diameter had the opposite effect, for the same reason.

The temperature responses of the cladding and control blade components for the $9.6 \mathrm{~cm}$ plane are shown in Figures 5.2 .7 and 5.2.8, respectively. Changing the debris diameter resulted in no change in cladding and control blade temperature behavior.

In summary, the component temperatures were not sensitive to changes in debris diameter, but hydrogen production was quite sensitive to this parameter. The value used for this parameter should reflect the expected debris geometry and composition.

\subsection{Oxidation Studies}

Two studies were performed to investigate the oxidation modeling in the MEL$\mathrm{COR}$ core package. The two studies concern $\mathrm{B}_{4} \mathrm{C}$ oxidation and the transition temperature between slow and fast oxidation reaction rates.

\subsection{1 $\quad \mathrm{B}_{4} \mathrm{C}$ Oxidation Model}

The $\mathrm{B}_{4} \mathrm{C}$ oxidation model in the MELCOR core package is turned off by default because of difficulties encountered with the model [17]. The model was left off in the base case MELCOR model for DF-4. For this sensitivity study, this model was turned on by activating a flag on the CORTST01 input record. 


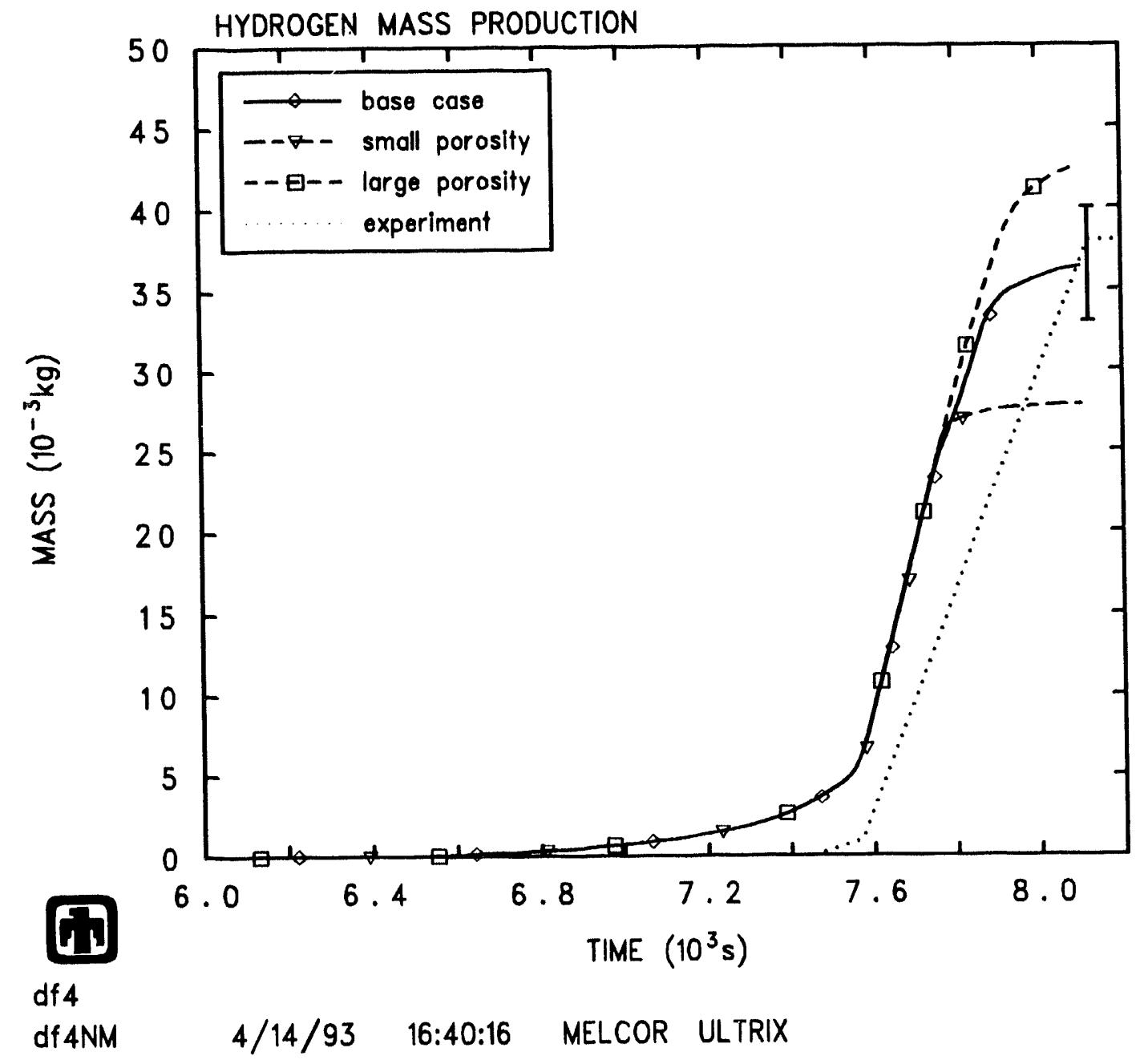

Figure 5.2.1. Hydrogen production for the debris porosity sensitivity study, compared to base case and measured values. 


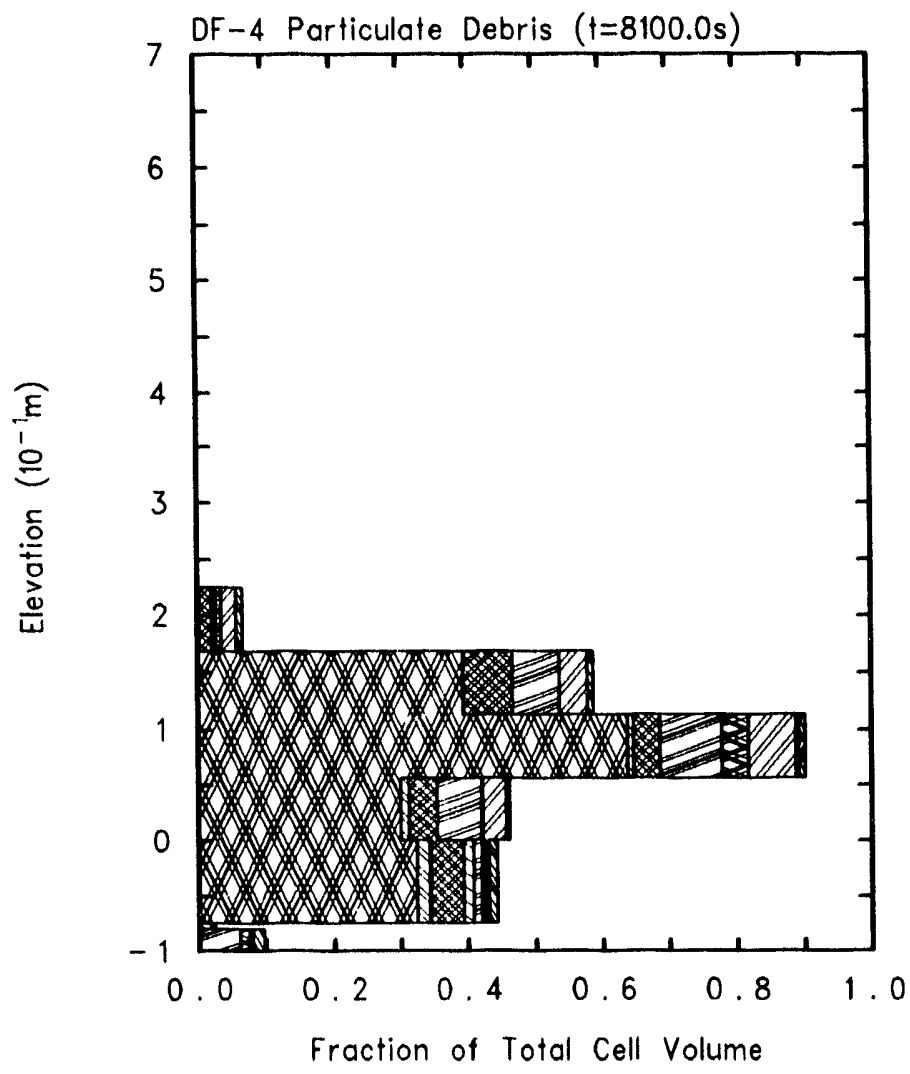

Intoct:

$\mathrm{vO}_{2}$

$\mathrm{Zr}$

$\geqslant$ ss

$\mathrm{ZrO}_{2}$

$\forall$ ssox

CRP

N $I N C$

Cong Debris:

$\mathrm{UO}_{2}$ in Deb

$\sum \mathrm{Zr}$ in Deb

SS in Deb

VIII $\mathrm{ZrO}_{2}$ in $\mathrm{Deb}$

Ssox in Deb

CRP in Deb

INC in Deb

Figure 5.2.2. Particulate debris component material locations at 8100 seconds for the "small" case of the debris porosity sensitivity study. 


\section{$x$}

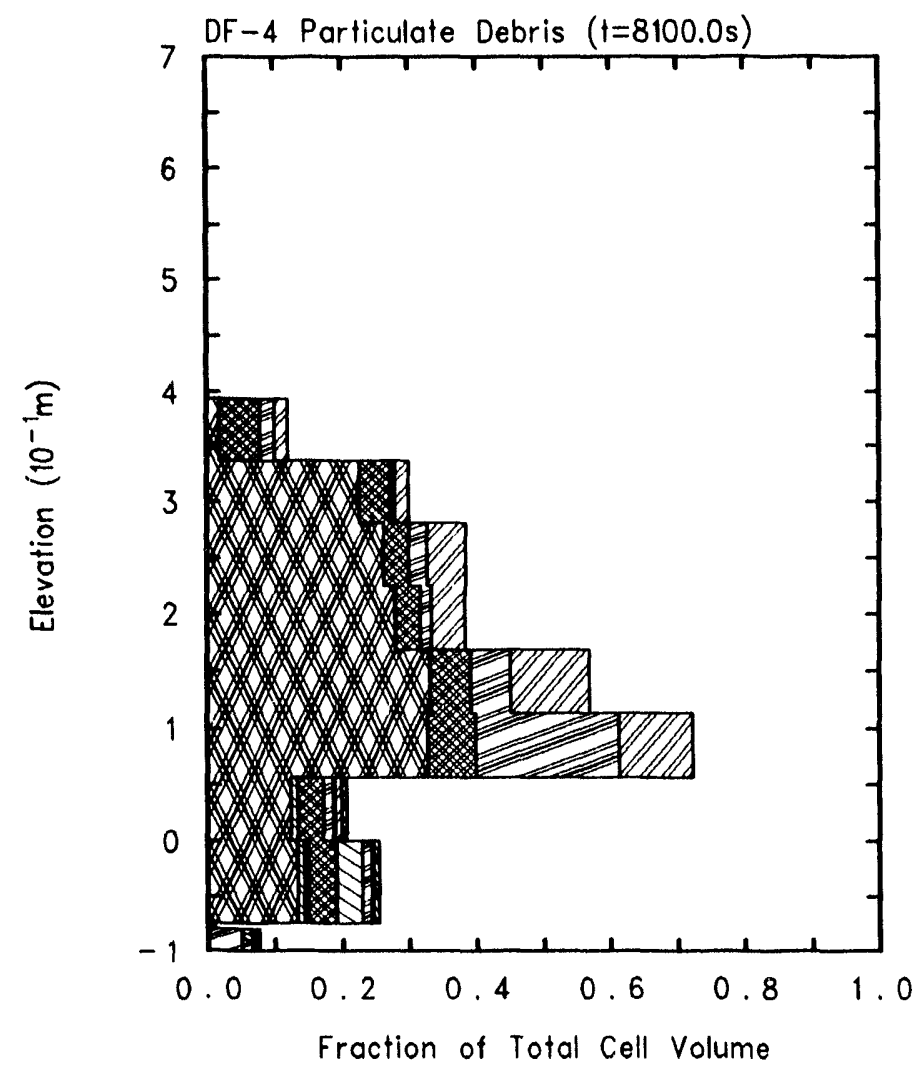

Intact:

$\mathrm{vO}_{2}$

$\mathrm{Zr}$

2 ss

$\mathrm{ZrO}_{2}$

ssox

6 CRP

算 $I N C$

Cong Debris:

$\mathrm{UO}_{2}$ in Deb

Zr in Deb

SS in Deb

VID $\mathrm{ZrO}_{2}$ in Deb

SSOx in Deb

CRP in Deb

INC in Deb

Fraction of Total Cell Volume

Figure 5.2.3. Particulate debris component material locations at 8100 seconds for the "large" case of the debris porosity sensitivity study. 


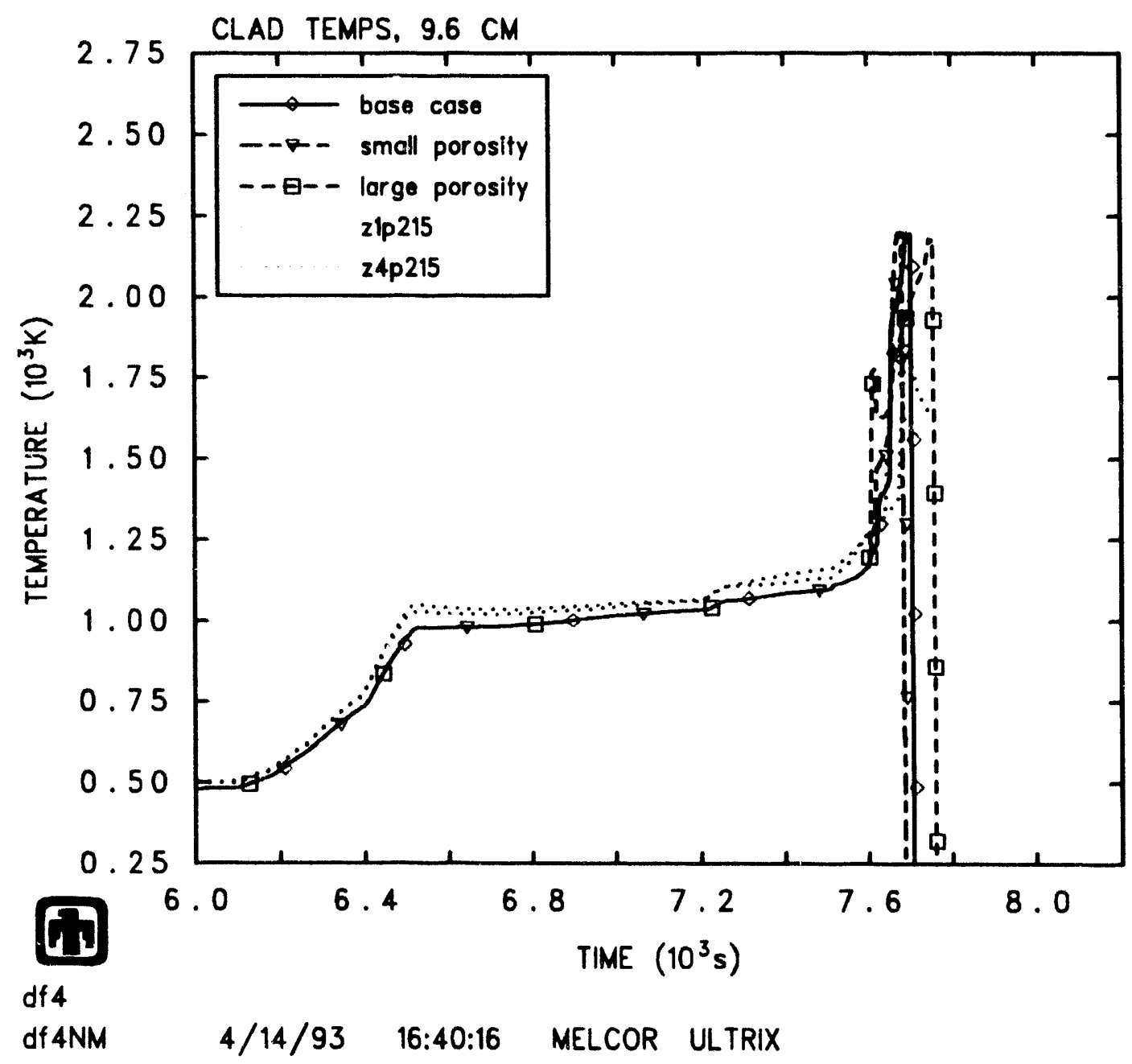

Figure 5.2.4. Cladding temperature response at the $9.6 \mathrm{~cm}$ plane for the debris porosity sensitivity study, compared to base case and measured values. 


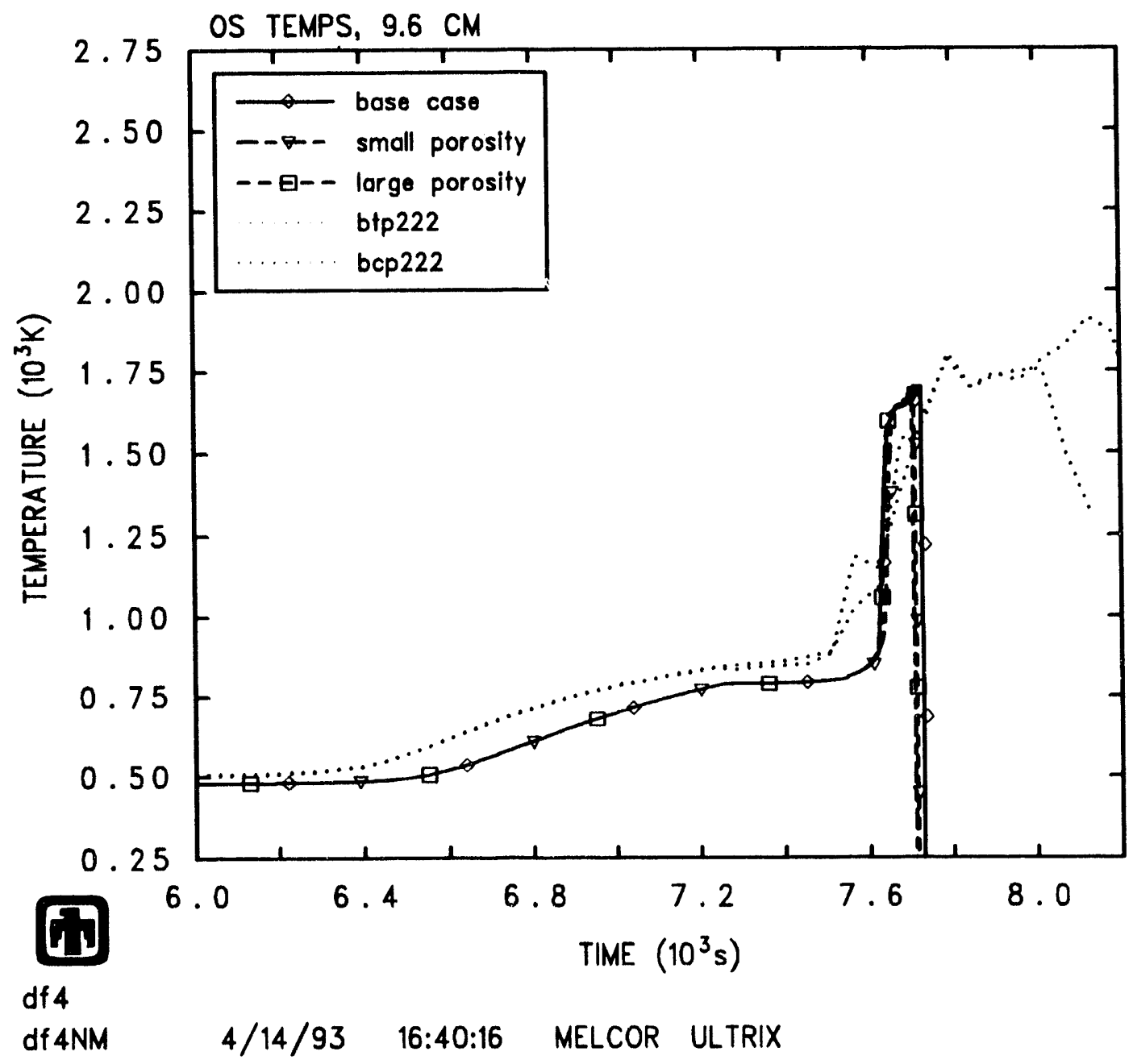

Figure 5.2.5. Control blade temperature response at the $9.6 \mathrm{~cm}$ plane for the debris porosity sensitivity study, compared to base case and measured values. 


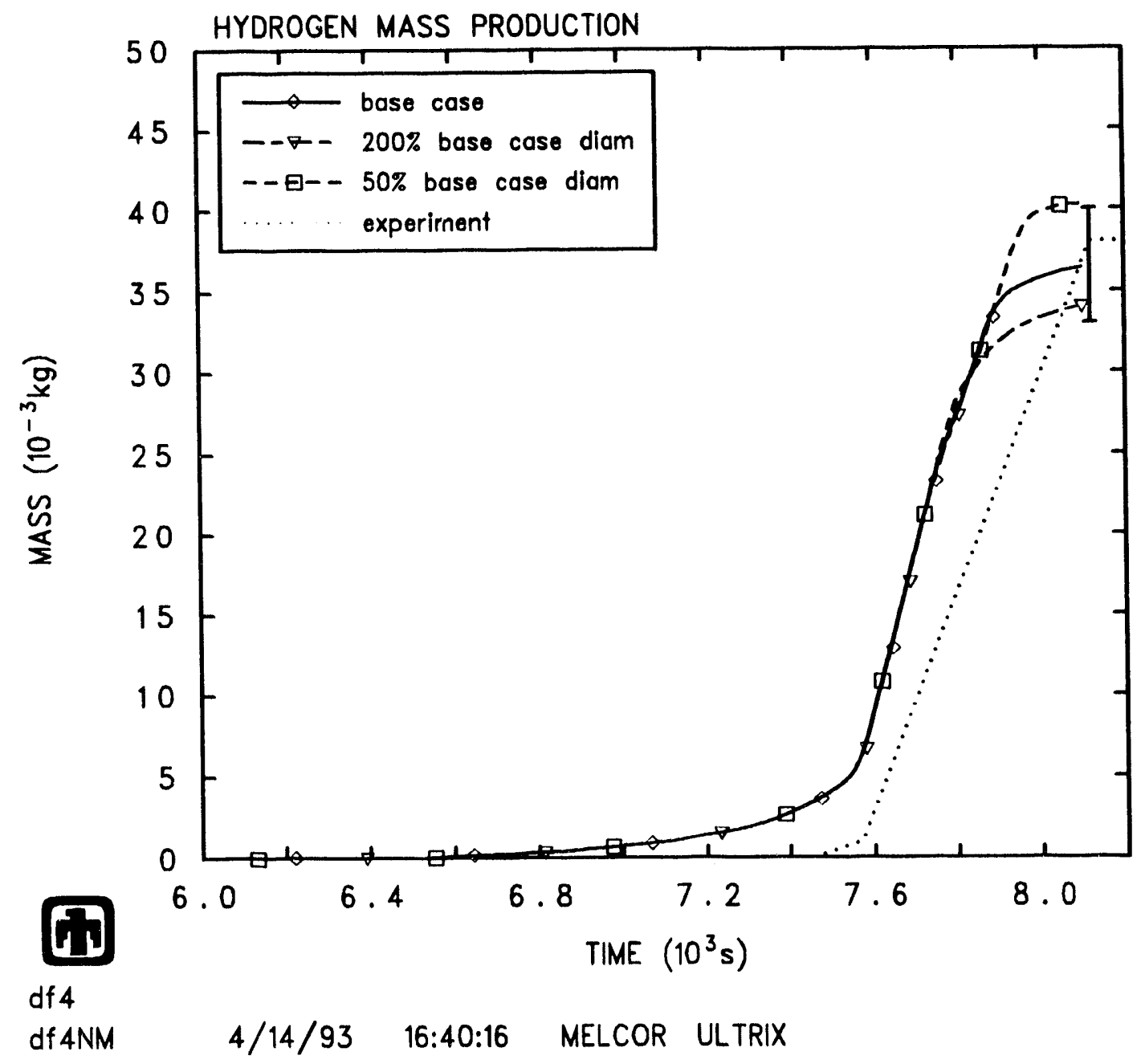

Figure 5.2.6. Hydrogen production for the debris diameter sensitivity study, compared to base case and measured values. 


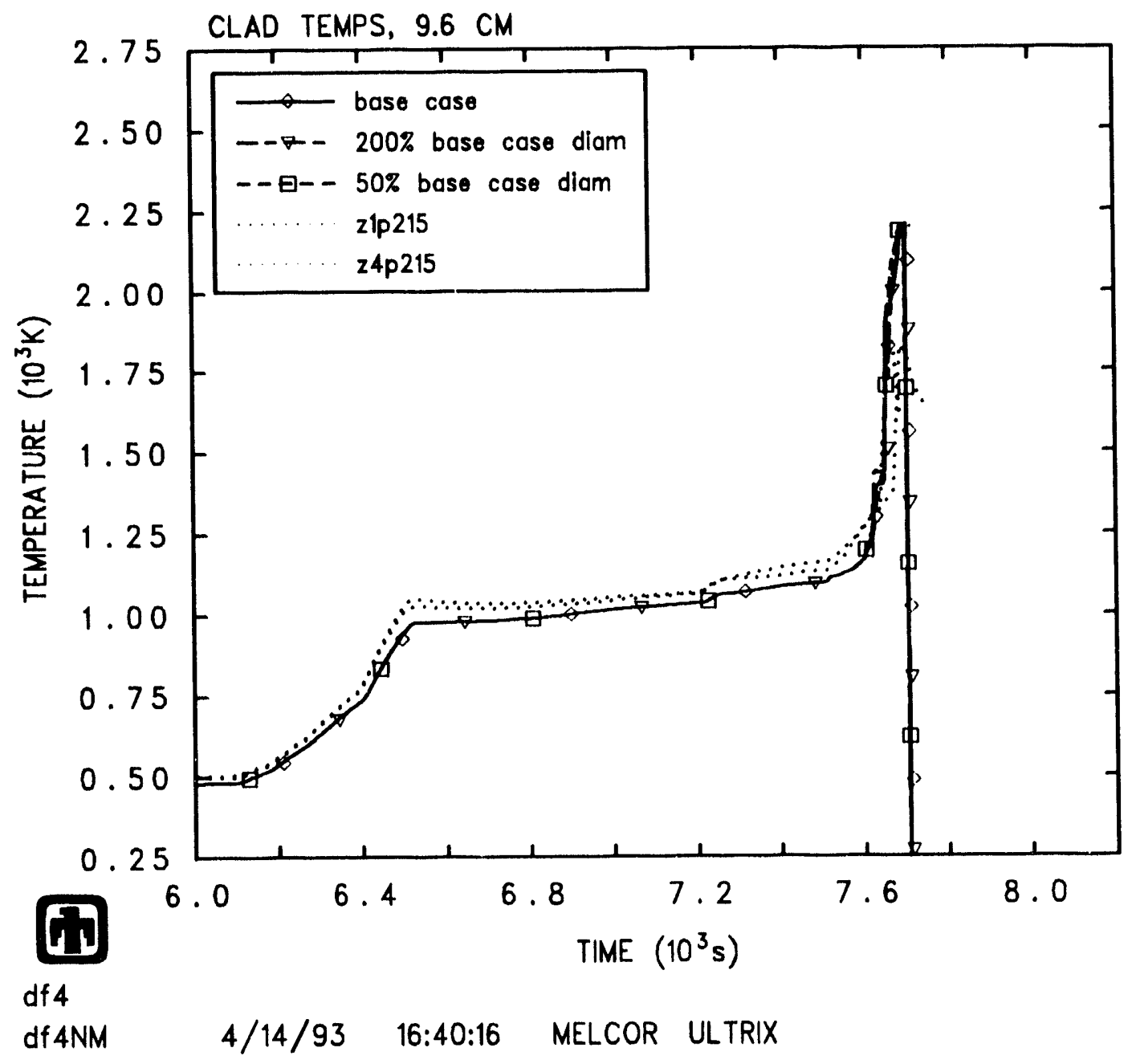

Figure 5.2.7. Cladding temperature response at the $9.6 \mathrm{~cm}$ plane for the debris diameter sensitivity study, compared to base case and measured values. 


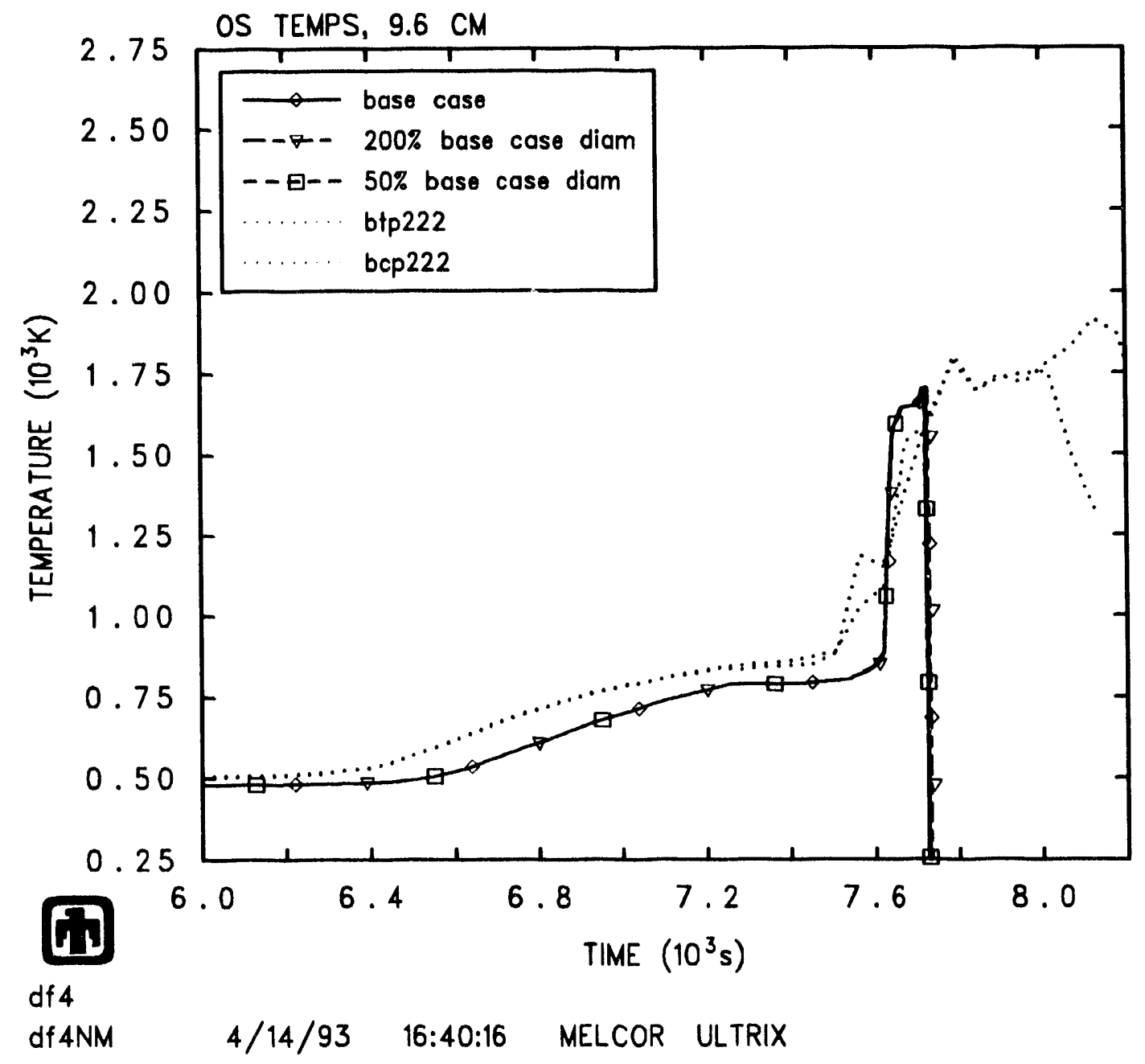

Figure 5.2.8. Control blade temperature response at the $9.6 \mathrm{~cm}$ plane for the debris diameter sensitivity study, compared to base case and measured values. 
In the MELCOR implementation of $\mathrm{B}_{4} \mathrm{C}$ oxidation, the poison material did not begin oxidizing until the other structure component reached the stainless steel melting temperature. In effect this modeled breach of the stainless steel tubes which held the $\mathrm{B}_{4} \mathrm{C}$. The results from this sensitivity study, though, show that the $\mathrm{B}_{4} \mathrm{C}$ model was never exercised, even when the model was enabled. This is because, with the new eutectic model enabled, all of the $\mathrm{B}_{4} \mathrm{C}$ was consumed in the eutectic reaction with stainless steel before the other structure component could reach the stainless steel melting temperature (this assumes there was enough stainless steel available to totally consume the available $\mathrm{B}_{4} \mathrm{C}$ in a eutectic reaction). The $\mathrm{B}_{4} \mathrm{C}$ oxidation model does not calculate oxidation for $\mathrm{B}_{4} \mathrm{C}$ in the liquid state. This situation would probably exist in any realistic BWR input model, which would have the eutectic model active. So, in conclusion, without the capability of oxidizing $\mathrm{B}_{4} \mathrm{C}$ in a eutectic mixture, the $\mathrm{B}_{4} \mathrm{C}$ oxidation model is of little use to the MELCOR user for modeling BWR cores.

\subsubsection{Oxidation Reaction Rate Transition Temperature}

Oxidation is modeled in MELCOR using a parabolic rate equation. For the zircaloy $-\mathrm{H}_{2} \mathrm{O}$ reaction, the rate constant is calculated using the Urbanic-Heidrich correlation [18]:

$$
\begin{aligned}
K(T) & =29.6 \exp \left(\frac{-16820.0}{T}\right) \text { for } \mathrm{T}<\mathrm{T}_{\text {trans }} \\
& =87.9 \exp \left(\frac{-16610.0}{T}\right) \text { for } \mathrm{T} \geq \mathrm{T}_{\text {trans }}
\end{aligned}
$$

The transition temperature, $T_{\text {trans }}$, has a default value of $1853 \mathrm{~K}$, and is adjustable using sensitivity coefficient 1001 in the COR package. In this sensitivity study, values of $1550 \mathrm{~K}, 1650 \mathrm{~K}$ and the default value of $1853 \mathrm{~K}$ were compared with the DF-4 base case which used a transition temperature of $1750 \mathrm{~K}$.

The cladding and control blade temperatures for the $36.8 \mathrm{~cm}$ plane for these three cases are compared to base case and measured results in Figures 5.3.1 and 5.3.2, respectively. The trends in these figures are consistent with the adjustments to the oxidation transition temperature. That is, when the oxidation transition temperature was decreased, rapid oxidation started earlier and resulted in earlier component failure.

The hydrogen production for these three cases in this study is compared to the base case and measured results in Figure 5.3.3. The hydrogen production for each case was also consistent with the trends in transition temperatures, where the lower transition temperature cases resulted in earlier and more hydrogen production. It is interesting to note that the $1853 \mathrm{~K}$ case showed much less hydrogen production. This can be understood by looking at Figure 5.3.1, which shows that in this case the clad reached its melting temperature much sooner after starting rapid oxidation than the other cases, which means it had less time to produce hydrogen at the increased rate.

The oxidation reaction rate transition temperature used in the base case model, $1750 \mathrm{~K}$, resulted in the best overall agreement with experimental data, in terms of temperatures, failure timing and overall hydrogen production. The results showed 
sensitivity to this parameter, and it is recommended that this parameter be investigated as a part of any MELCOR modeling effort. (Both the LOFT LP-FP-2 [11] and the CORA-13 [13] assessments adjusted this parameter in the base case models.)

\subsection{Eutectic Model Studies}

The materials interaction (eutectic) model in the core package was a recent addition to the MELCOR code [7]. This model represents the interaction between certain materials in BWR and PWR reactor cores which results in liquid mixtures forming well below their normal individual melting temperatures. This phenomenon was of concern because it could result in control poison escaping the core before significant fuel relocation, possibly resulting in a reactivity transient. Two studies were done to test the eutectic model in MELCOR. These studies concerned the use of the eutectic model and the eutectic temperature of the $\mathrm{B}_{4} \mathrm{C}$-stainless stcel reaction.

\subsubsection{Deactivate Eutectic Model}

The base case MELCOR model for DF-4 used the new MELC $\checkmark$ R 1.8 .2 eutectic model, which is activated using input record COR00006. In this sensitivity study, the eutectic model was reset to its default state, where it is inactive.

The largest difference in results due to the eutectic model was evident in the relocation behavior of the $\mathrm{B}_{4} \mathrm{C}$ between $7500-7600$ seconds. The distribution of control blade materials in the other structure component at 7550 seconds for the deactivated eutectic model calculation is shown in Figure 5.4.1. This figure can be compared to the other structure component material distribution plot in Figure 4.2.4. Figure 4.2.4 shows that the eutectic model calculated eutectic interaction and relocation of $\mathrm{B}_{4} \mathrm{C}$ from core cells $109 \cdots 112$ (see Table 3.2.2). According to the base case model, at 7550 seconds there was no $\mathrm{B}_{4} \mathrm{C}$ material left in cells $110-112$ (between $34 \mathrm{~cm}$ and $50.5 \mathrm{~cm}$ ). The case with the eutectic model deactivated showed no significant relocation of $\mathrm{B}_{4} \mathrm{C}$ from any level at 7550 seconds. The material distribution for the deactivated eutectic model case at 7600 seconds is shown in Figure 5.4.2, for comparison to the base case results in Figure 4.2.5. At this time, the other structure component had failed in cells 109112 in both calculations. After failure of the substrate material (stainless steel), in the case with no eutectics interaction, the $\mathrm{B}_{4} \mathrm{C}$ was converted to particulate debris and was relocated to the bottom of the core in a manner similar to the way $\mathrm{UO}_{2}$ relocated. This shows the value of using the eutectic model in MELCOR; it is clear from both the temperature measurements and the video record of the DF-4 experiment that the control blade material melted and relocated at a temperature lower than the stainless steel melting temperature, and that it relocated (candled) in the liquid phase.

The final bundle state for this sensitivity study is shown in Figure 5.4.3. This figure can be compared with the base case results, shown in Figure 4.2.10. In the case with no cutectic modeling, more of the fuel and canister components survived intact, instead of being converted to particulate debris. This shows less dissolution of these 


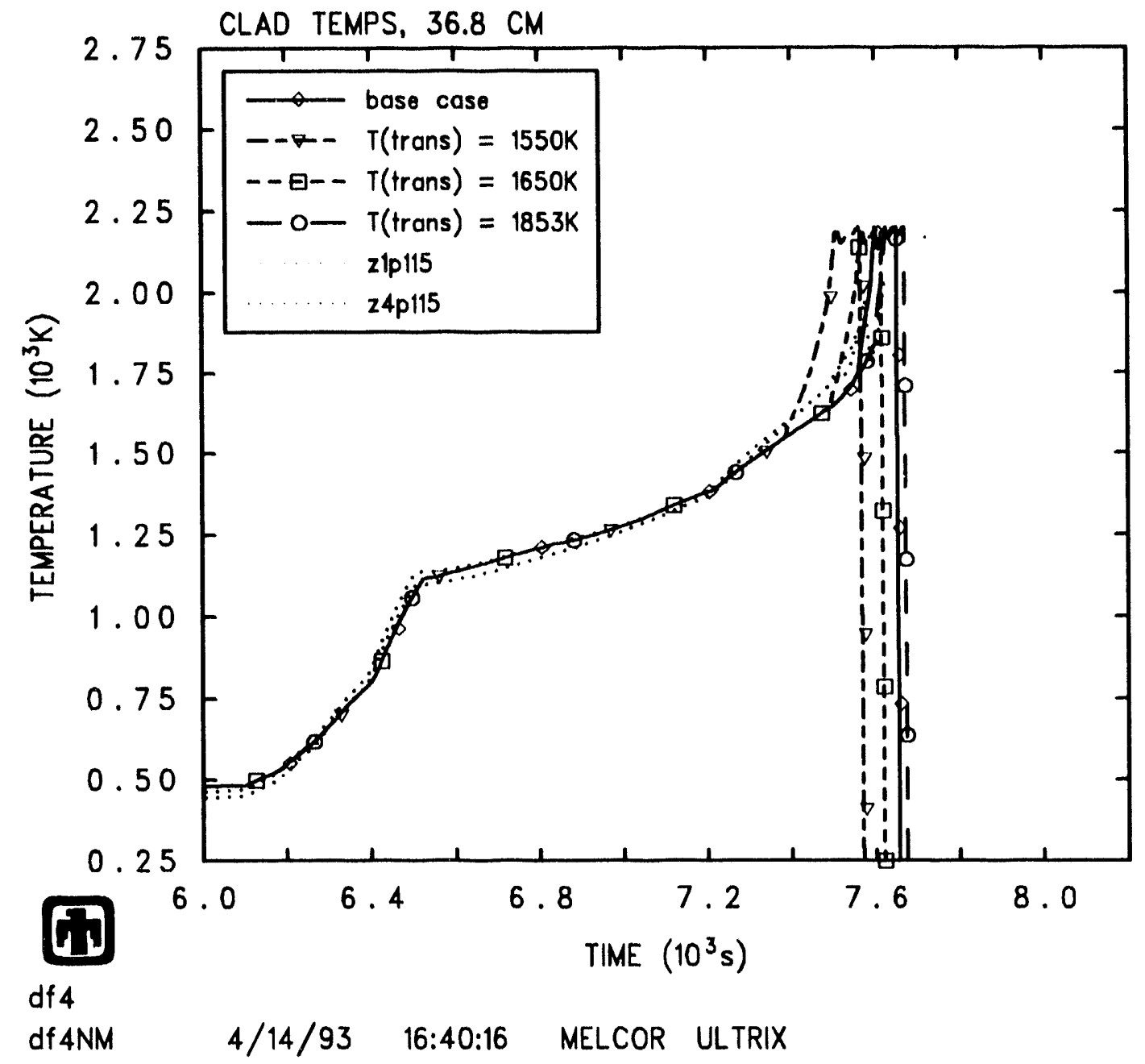

Figure 5.3.1. Cladding temperature response at the $36.8 \mathrm{~cm}$ plane for the oxidation reaction rate transition temperature sensitivity study, compared to base case and measured values. 


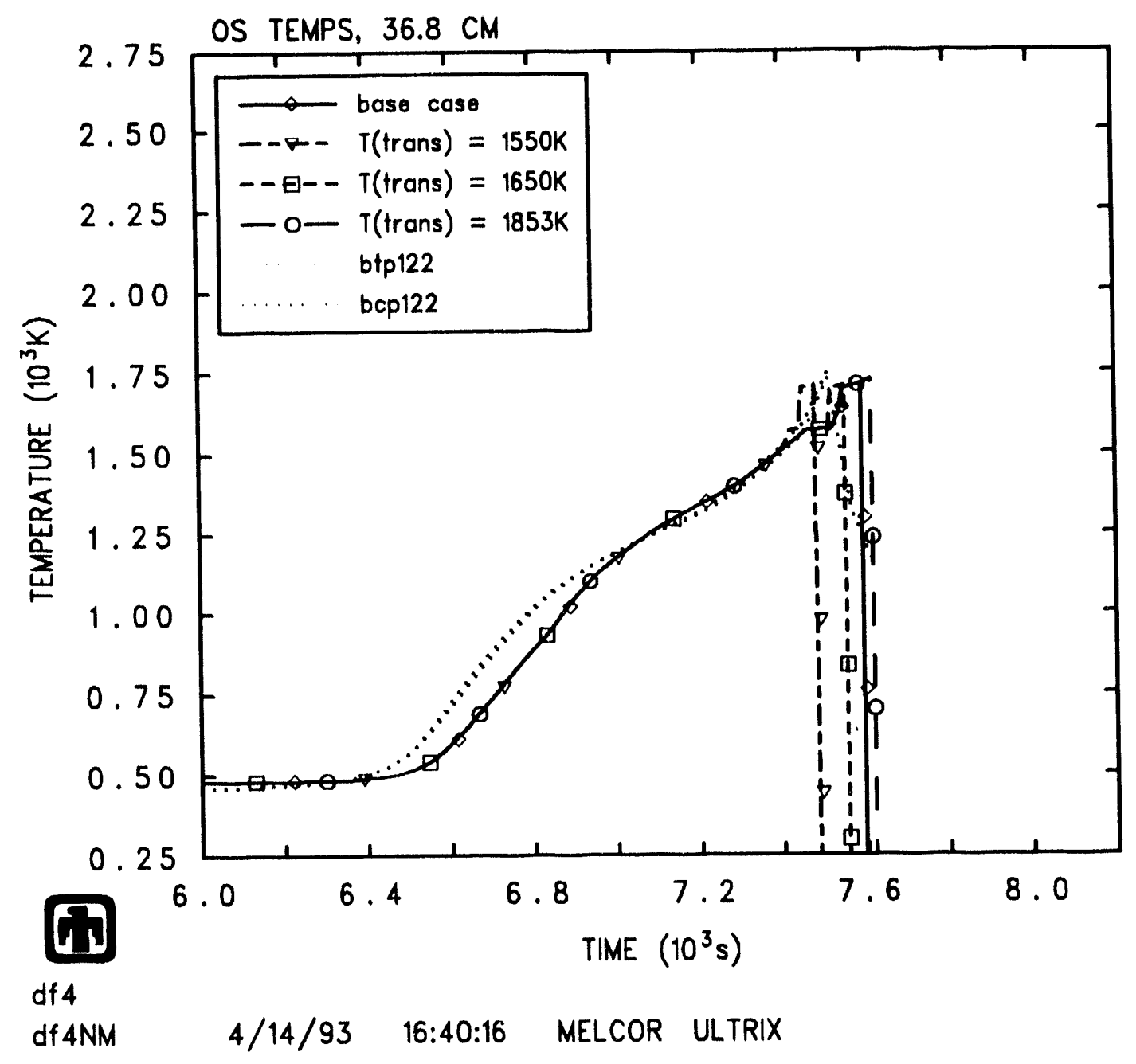

Figure 5.3.2. Control blade temperature response at the $36.8 \mathrm{~cm}$ plane for the oxidation reaction rate transition temperature sensitivity study, compared to base case and measured values. 


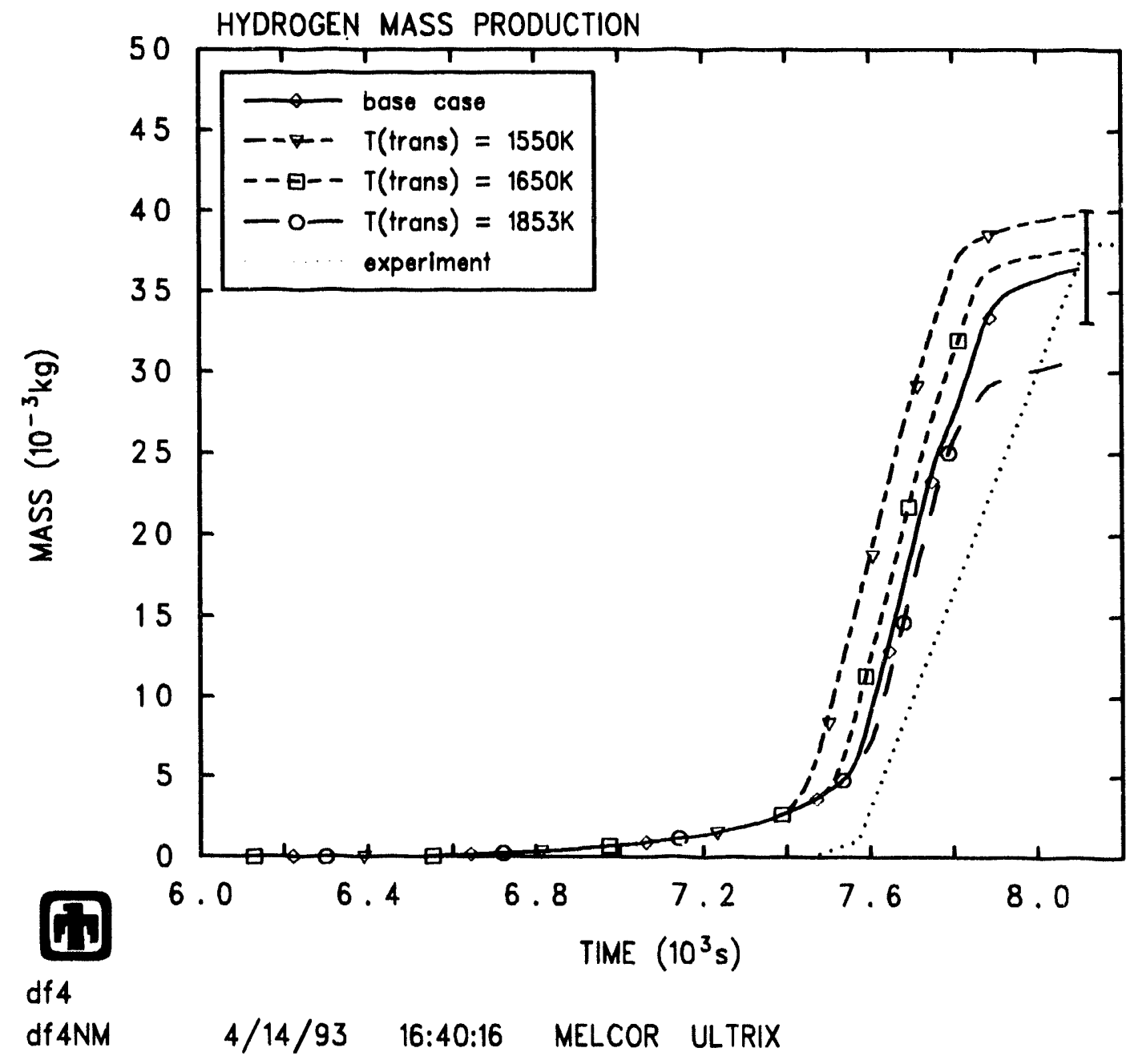

Figure 5.3.3. Hydrogen production for the oxidation reaction rate transition temperature sensitivity study, compared to base case and measured values. 
components by debris materials already residing at those levels. Eutectic dissolution of the fuel and cladding component moves material from that component to the particulate debris component. Moving zircaloy to the particulate debris component made it available for oxidation, whereas in the fuel and cladding component the zircaloy had been covered by an oxide layer. Thus, deactivating the new eutectics model left more material in the fuel and cladding component, making it unavailable for oxidation and reducing the total amount of hydrogen produced. Figure 5.4.4, the hydrogen production for the no eutectics interaction case, shows that this was indeed the case.

The control blade temperature with the eutectic model inactive at the $36.8 \mathrm{~cm}$ plane is compared with the base case and measured results in Figure 5.4.5. The results without eutectic modeling were the same until the control blade reached the eutectic temperature of $1570 \mathrm{~K}$ (this was the value used in the base case model). The eutectic model held the control blade at this temperature to model the $\mathrm{B}_{4} \mathrm{C}$-stainless steel interaction, while with no eutectic modeling the control blade temperature rose to the stainless steel melting temperature of $1700 \mathrm{~K}$.

The cladding temperatures at the $36.8 \mathrm{~cm}$ and $25.4 \mathrm{~cm}$ planes are compared with base case and measured results in Figures 5.4.6 and 5.4.7, respectively. At the 25.4 cm plane, deactivating the cutectic model resulted in earlier failure of the cladding. This is becatse there was less material refrozen onto the control blade at that level, providing less of a heat sink for the fucl rod. The change in failure time was less than 50 seconds, though, so this was a small effect. The effect on temperature and failure timing of the control blade at the $36.8 \mathrm{~cm}$ level was even more slight.

This study clearly shows that the eutectic model should be used in future MELCOR BWR models. The temperature results were not very sensitive to the use of this model, but the relocation behavior of control blade materials was strongly affected by the cutectic model. Eutectic dissolution reactions were also important in determining the final debris state.

\subsubsection{Stainless Steel- $\mathrm{B}_{4} \mathrm{C}$ Eutectic Interaction Temperature}

One of the most important models affecting control blade temperature and relocation behavior in DF-4 was the new materials interactions model in MELCOR [7]. This model accounts for the liquifaction of $\mathrm{B}_{4} \mathrm{C}$ and stainless steel at a temperature well below the melting temperature of either constituent, as well as interactions between other materials. In this sensitivity study, the eutectic temperature of the $\mathrm{B}_{4} \mathrm{C}$-stainless stcel mixture was varied by $\pm 50 \mathrm{~K}$ from its hase case model value of $1570 \mathrm{~K}$. This parameter is located in sensitivity coefficient array 1011 in MELCOR.

The control blade temperature response at the $36.8 \mathrm{~cm}$ plane was expected to show the effects of varying the eutectic temperature, because this was the first plane to show signs of eutectic interaction. The temperatures at this plane are compared with base case and measured results in Figure 5.4.8. As expected, lowering the eutectic interaction temperature caused the control blade to begin forming eutectic earlier. No matter what the eutectic temperature, the amount of energy required by the eutectic reaction between $B_{4}('$ and stainless steel is the same. Since the energy deposited in 


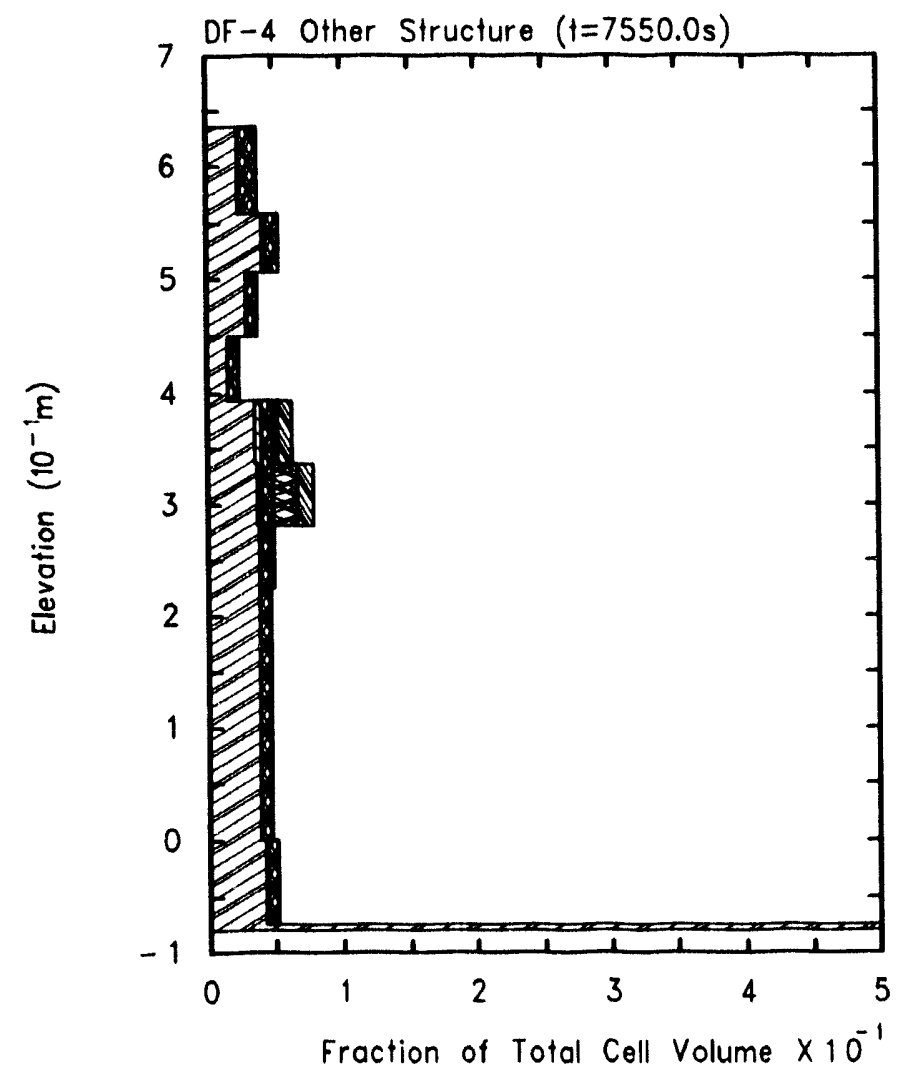

Intact:

$\mathrm{UO}_{2}$

$\mathrm{Zr}$

8 ss

\% $\mathrm{ZrO}_{2}$

$\Delta$ ssox

$\because$ CRP

筆 $I N C$

Cong Debris:

$\mathrm{UO}_{2}$ in $\mathrm{Deb}$

$\sum \mathrm{Zr}$ in Deb

SS in Deb

$\mathrm{ZrO}_{2}$ in Deb

Ssox in Deb

1. CRP in Deb

INC in Deb

Fraction of Total Cell Volume $\times 10^{-1}$

Figure 5.4.1. Other Structure component material locations at 7550 seconds for the eutectic model deactivation sensitivity study. 

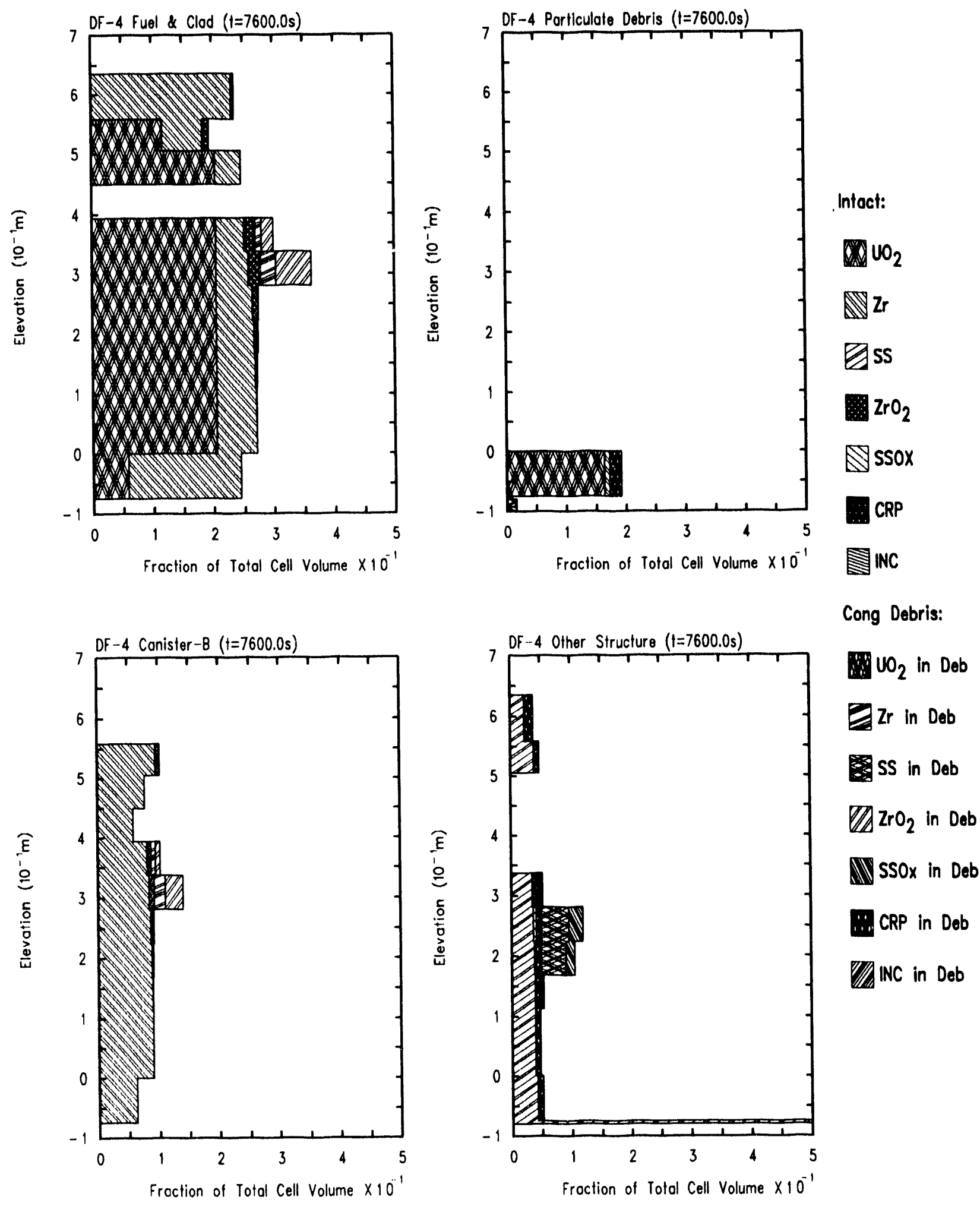

Figure 5.4.2. Locations of fuel and clad (upper left), particulate debris (upper right), canister-b (lower left) and other structure (lower right) component materials at 7600 seconds for the eutectic model deactivation sensitivity study. 

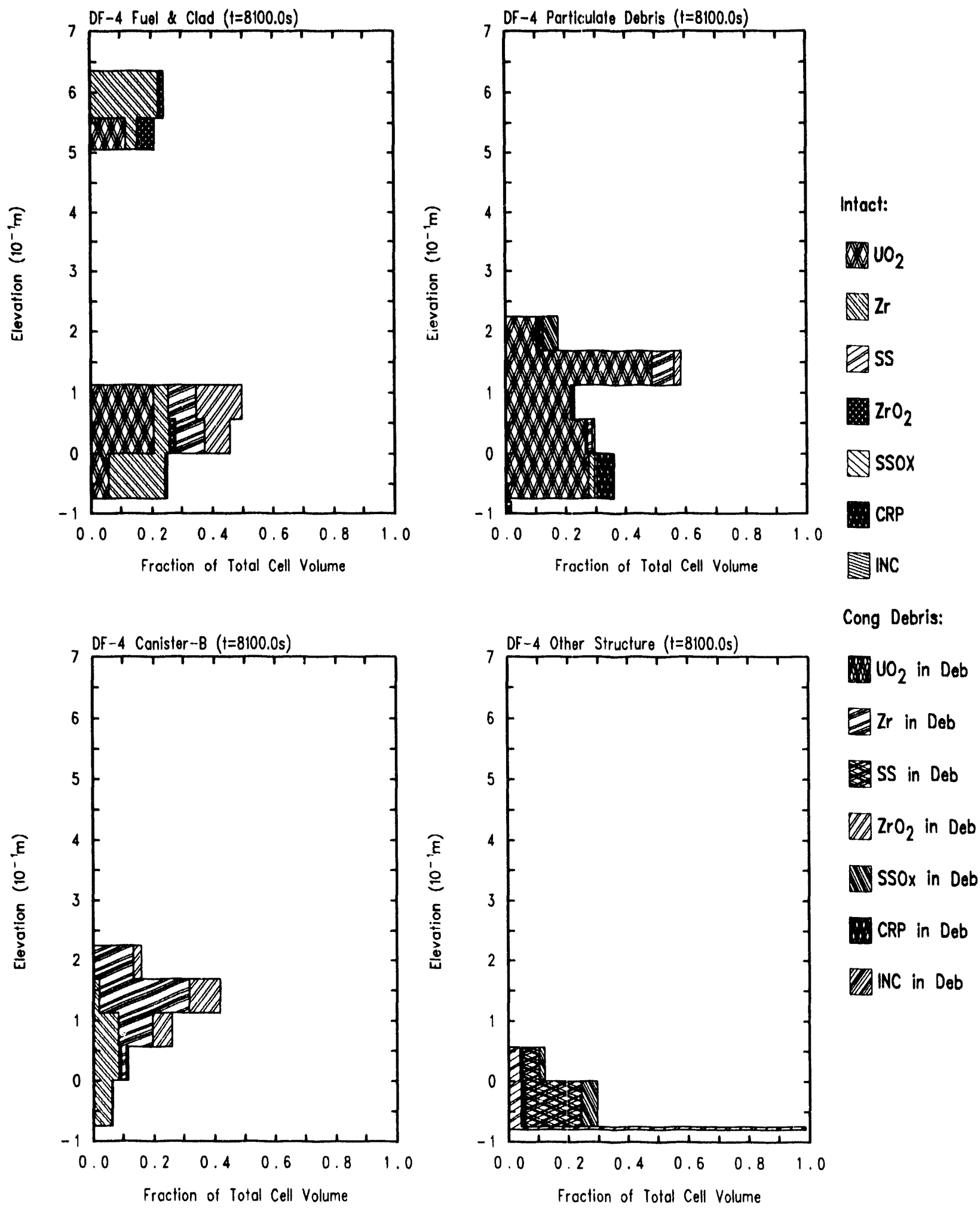

Figure 5.4.3. Material locations for the fuel and clad (upper left), particulate debris (upper right), canister-b (lower left) and other structure (lower right) components at 8100 seconds for the eutectic model deactivation sensitivity study. 


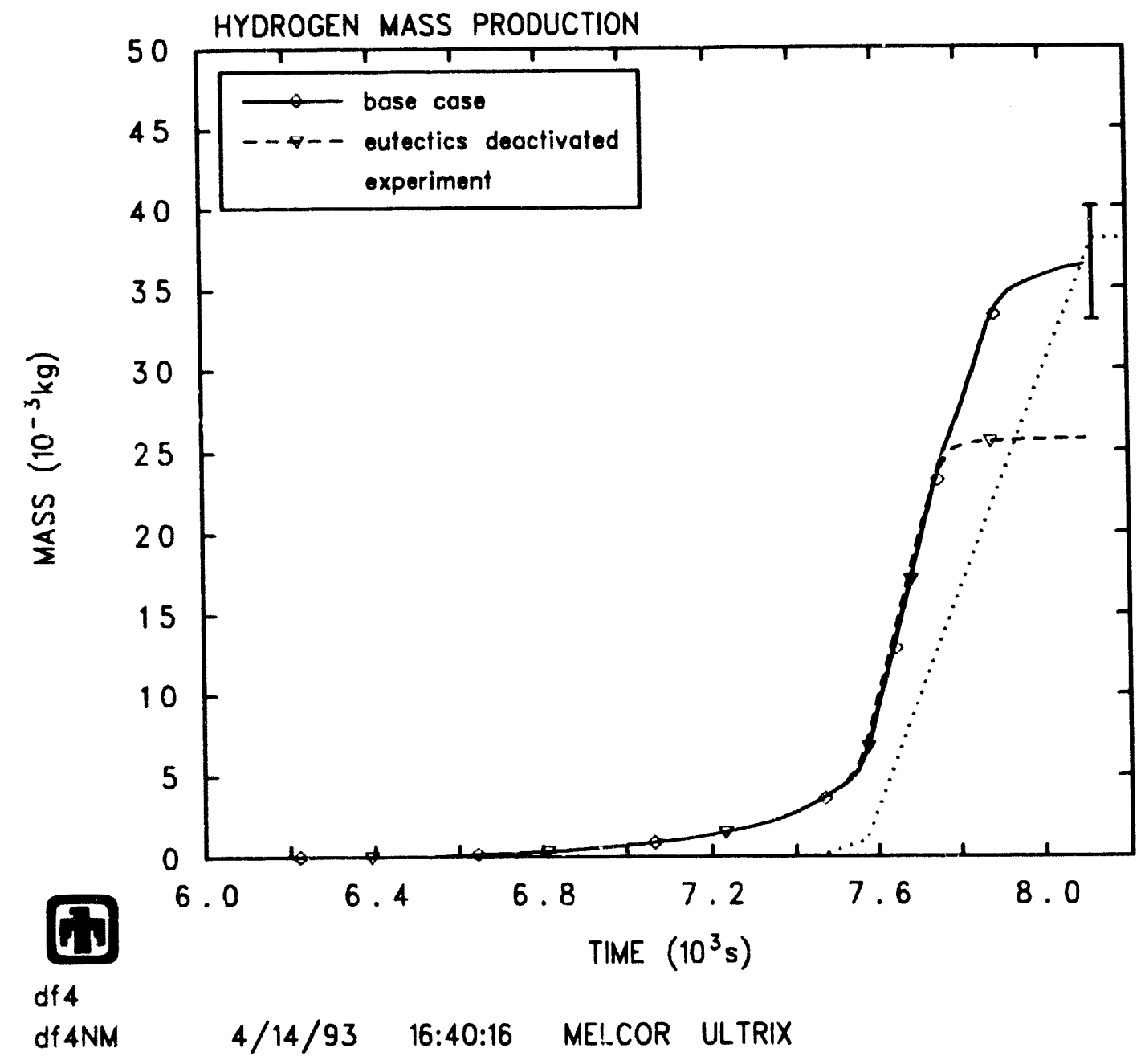

Figure 5.4.4. Hydrogen production for the eutectic model deactivation sensitivity study, compared to base case and measured values. 


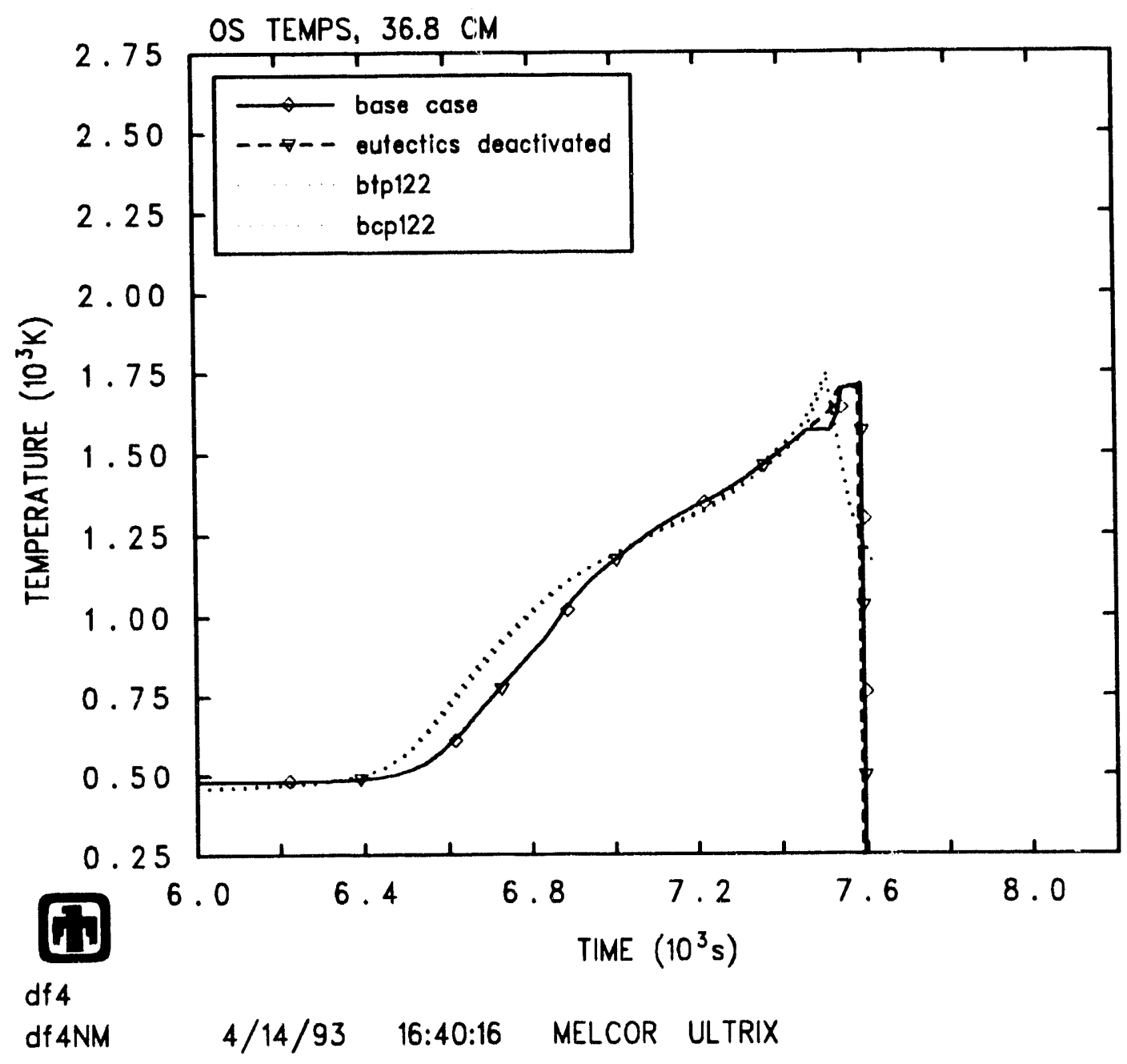

Figure 5.4.5. Control blade temperature response at the $36.8 \mathrm{~cm}$ plane for the eutectic model deactivation sensitivity study, compared to base case and measured values. 


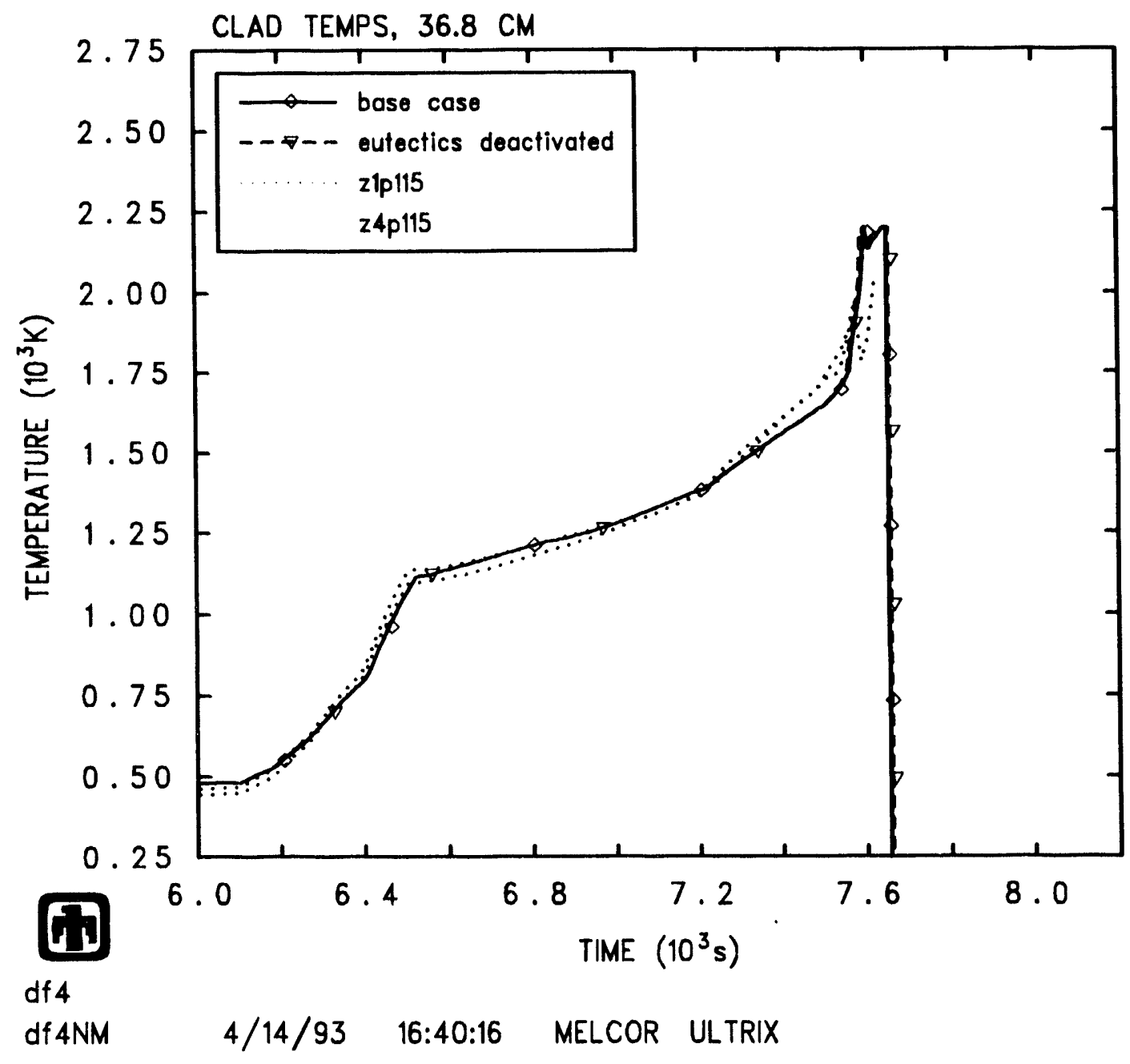

Figure 5.4.6. Cladding temperature response at the $36.8 \mathrm{~cm}$ plane for the eutectic model deactivation sensitivity study, compared to base case and measured values. 


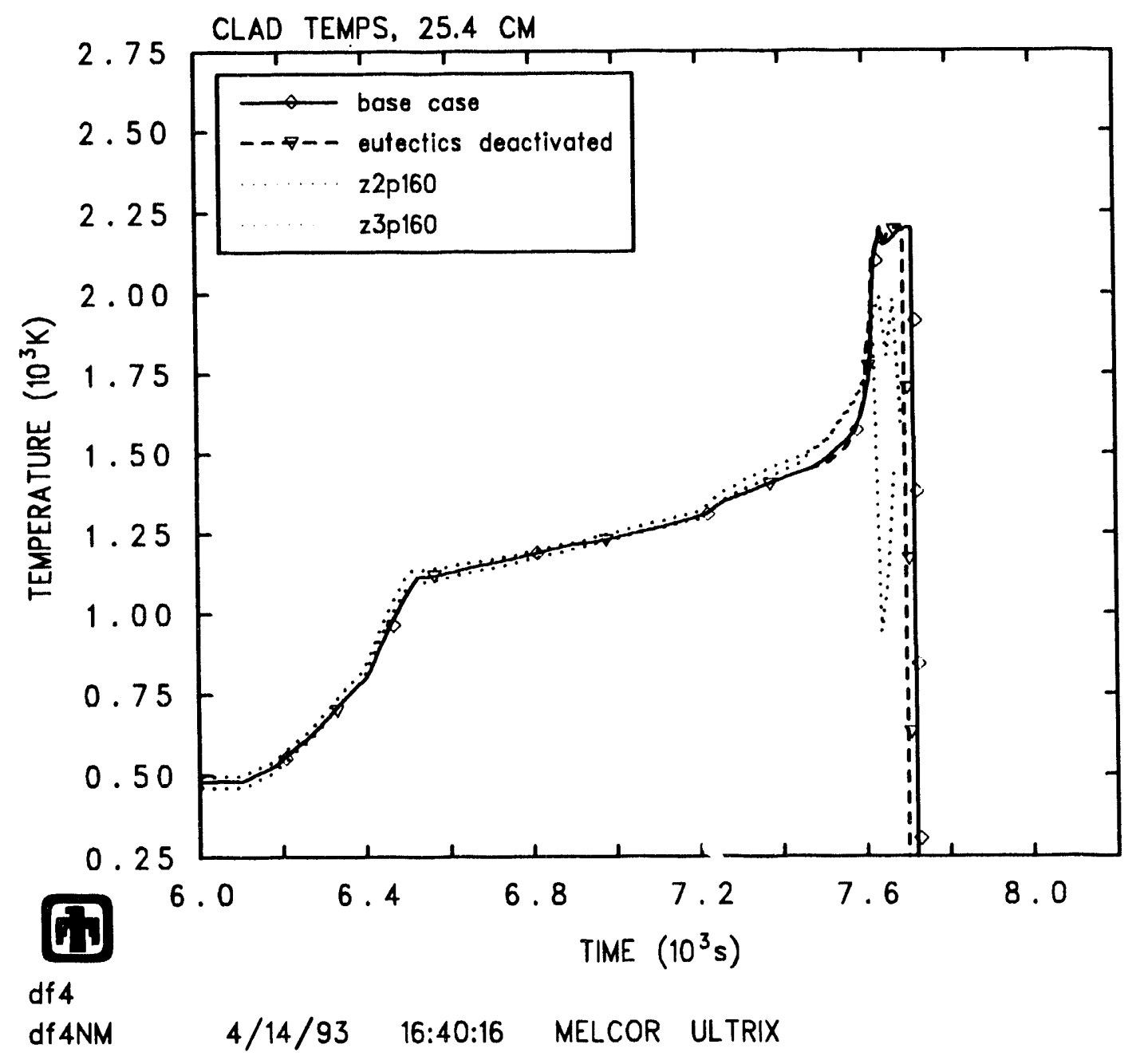

Figure 5.4.7. Cladding temperature response at the $25.4 \mathrm{~cm}$ plane for the eutectic model deactivation sensitivity study, compared to base case and measured values. 
the control blade during this time is the same for all cases, it follows that the failure timing for the control blade should not change significantly. This assumes a negligible difference in sensible heat addition below the eutectic interaction temperature for all cases.

The control blade temperature at the $25.4 \mathrm{~cm}$ plane showed the effects of earlier eutectic interaction at higher planes. Figure 5.4 .9 shows that relocating material arrived about 50 seconds earlier for the $1520 \mathrm{~K}$ case, and about the same amount of time later for the $1620 \mathrm{~K}$ case. Again, failure timing at this level was not affected.

The hydrogen production response, shown in Figure 5.4.10, was not affected by the variation in the eutectic temperature of the $\mathrm{B}_{4} \mathrm{C}$-stainless steel mixture.

The control blade material distributions in the other structure component for the $1520 \mathrm{~K}$ case at 7450 seconds are shown in Figure 5.4.11, to be compared with the base case control blade material distributions in Figure 4.2.2. Figure 5.4.11 shows earlier relocation of control blade materials as a result of decreasing the $\mathrm{B}_{4} \mathrm{C}$-stainless steel eutectic temperature. The control blade material relocations for the $1620 \mathrm{~K}$ case were delayed in time for the $1620 \mathrm{~K}$ case, in the same way that the relocations were moved forward in time for the $1520 \mathrm{~K}$ case.

\subsection{Thermal/Hydraulic Initial and Boundary Conditions}

Five sensitivity studies were done in the area of thermal/hydraulic initial and boundary conditions. Most of the studies were performed to investigate the effects of uncertainties or assumptions about the experimental initial and boundary conditions.

\subsubsection{Steam Mass Flow Rate}

The steam mass flow rate in the experiment was measured using a flow meter and data from this instrument was provided on one of the data channels. This data was used in the MELCOR base case model. For this sensitivity study, the mass flow rate was varied by $\pm 10 \%$. This was done by scaling the control function which calculated flow into the channel and bypass control volumes in the MELCOR DF-4 model.

The cladding temperature at the $36.8 \mathrm{~cm}$ plane for this study is compared with the base case and measured results in Figure 5.5.1. Increasing and decreasing the flow had the expected results of causing later and earlier failure, respectively. This was obviously the result of cooling proportional to the steam mass flow rate.

The hydrogen production behaved as expected during the early part of the experiment, as shown in Figure 5.5.2. However, the final amount of hydrogen produced for the $110 \%$ flow case was slightly less than the base case result. This suggests that the hydrogen production later in the experiment was not limited by flow. Rather, the oxidation reaction may have been limited by the characteristics of the debris. 


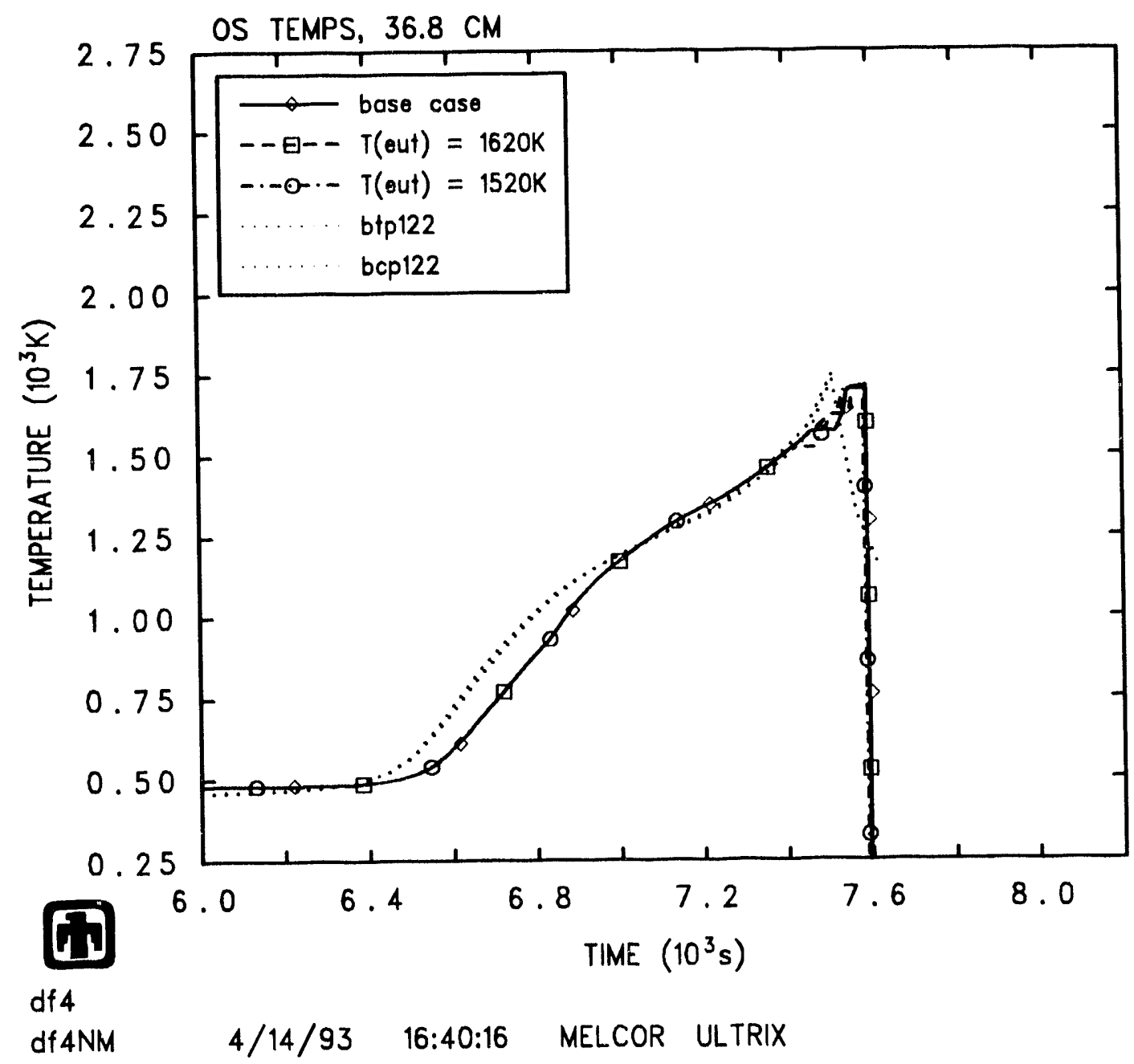

Figure 5.4.8. Control blade temperature response at the $36.8 \mathrm{~cm}$ plane for the $\mathrm{B}_{4} \mathrm{C}$-stainless steel eutectic temperature sensitivity study, compared to base case and measured values. 


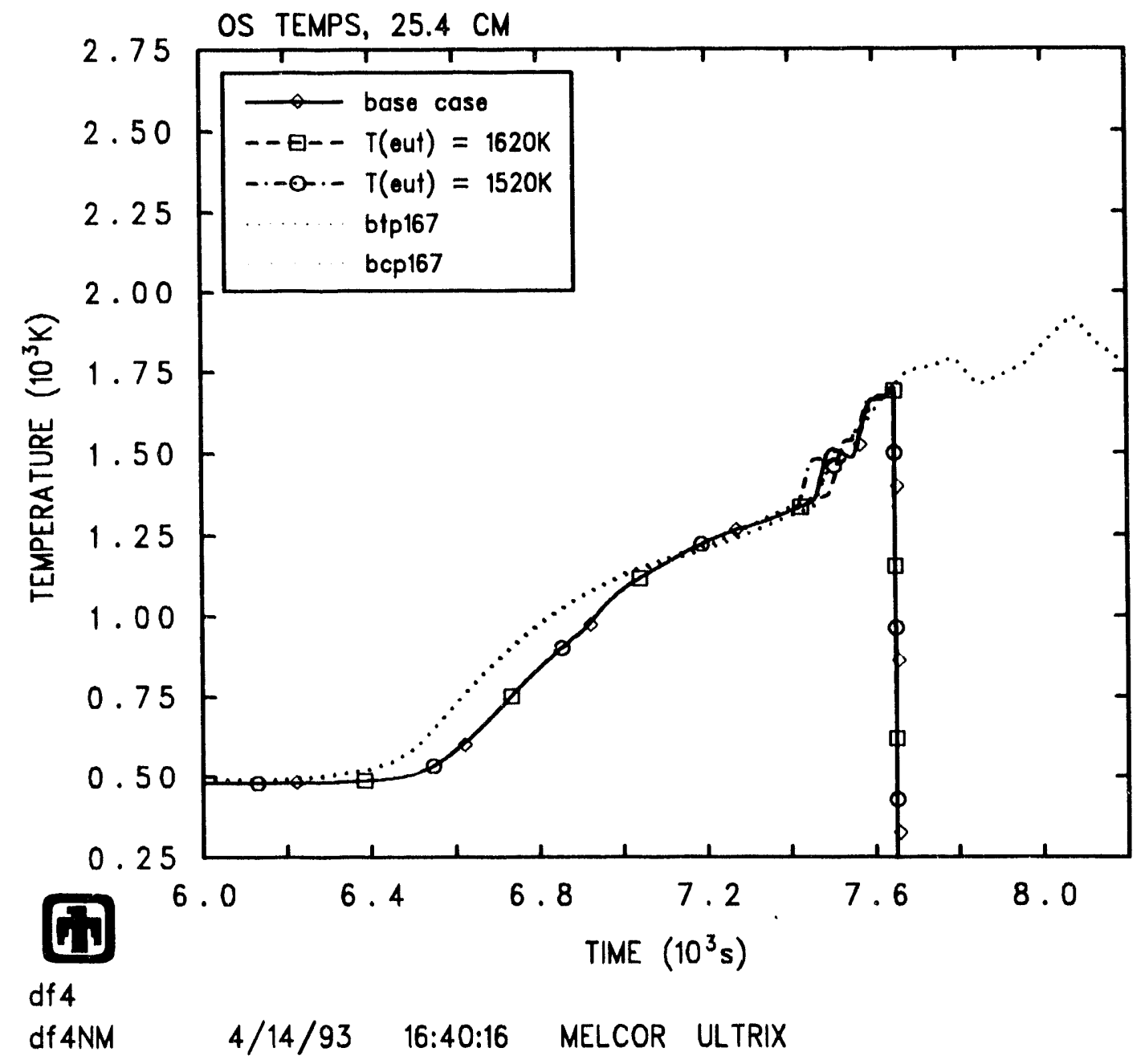

Figure 5.4.9. Control blade temperature response at the $25.4 \mathrm{~cm}$ plane for the $\mathrm{B}_{4} \mathrm{C}$-stainless steel eutectic temperature sensitivity study, compared to base case and measured values. 


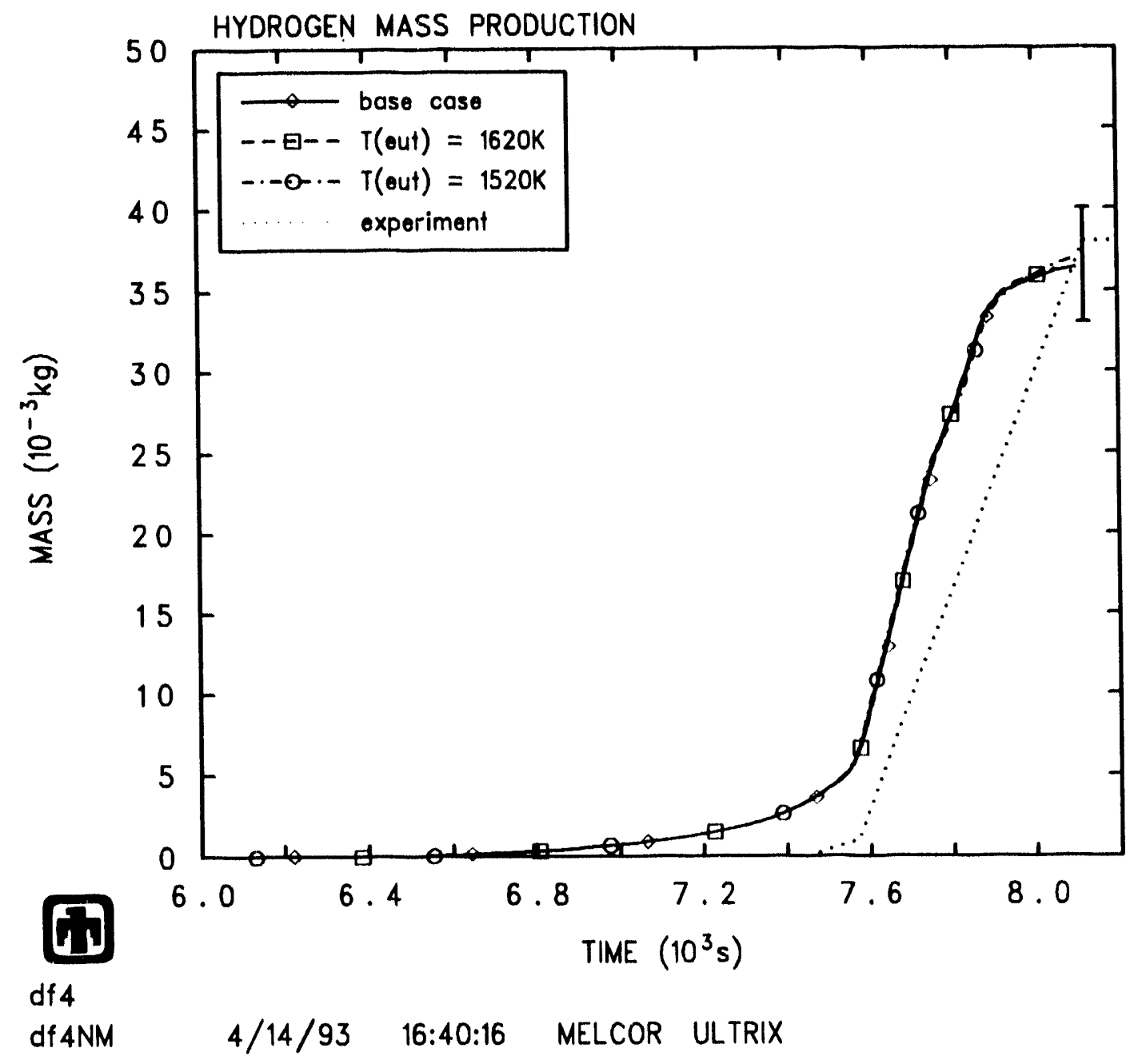

Figure 5.4.10. Hydrogen production for the $\mathrm{B}_{4} \mathrm{C}$-stainless stcel eutectic temperature sensitivity study, compared to base case and measured values. 


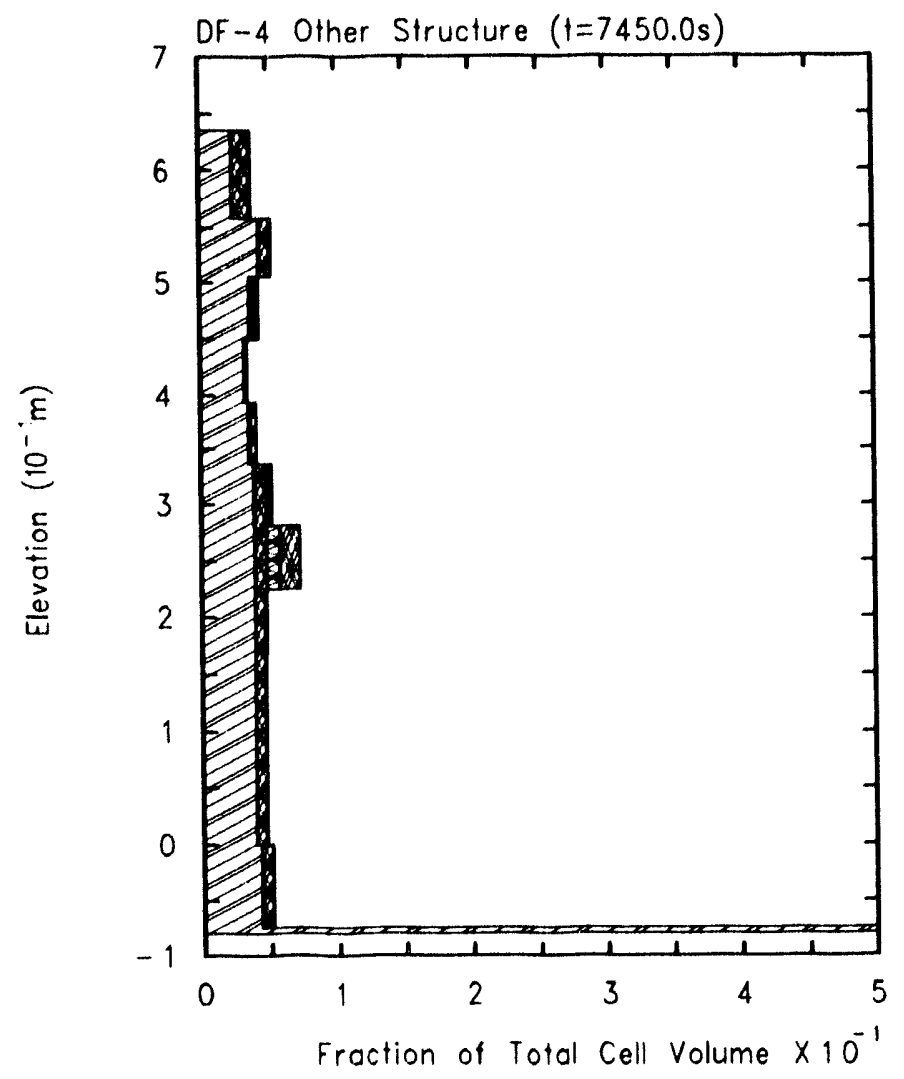

Intoct:

$\mathrm{NO}_{2}$

ed $2 r$

$\theta$ ss

$2 \mathrm{ZrO}_{2}$

83 ssox

$\because$ CRP

FinC

Cong Debris:

$\mathrm{UO}_{2}$ in Deb

$\sum 2 r$ in Deb

SS in Deb

VIIA $\mathrm{ZrO}_{2}$ in Deb

SSOx in Deb

CRP in Dob

INC in Deb

Figure 5.4.11. Other Structure component material locations at 7450 seconds for the $1520 \mathrm{~K}$ case of the eutectic temperature sensitivity study. 


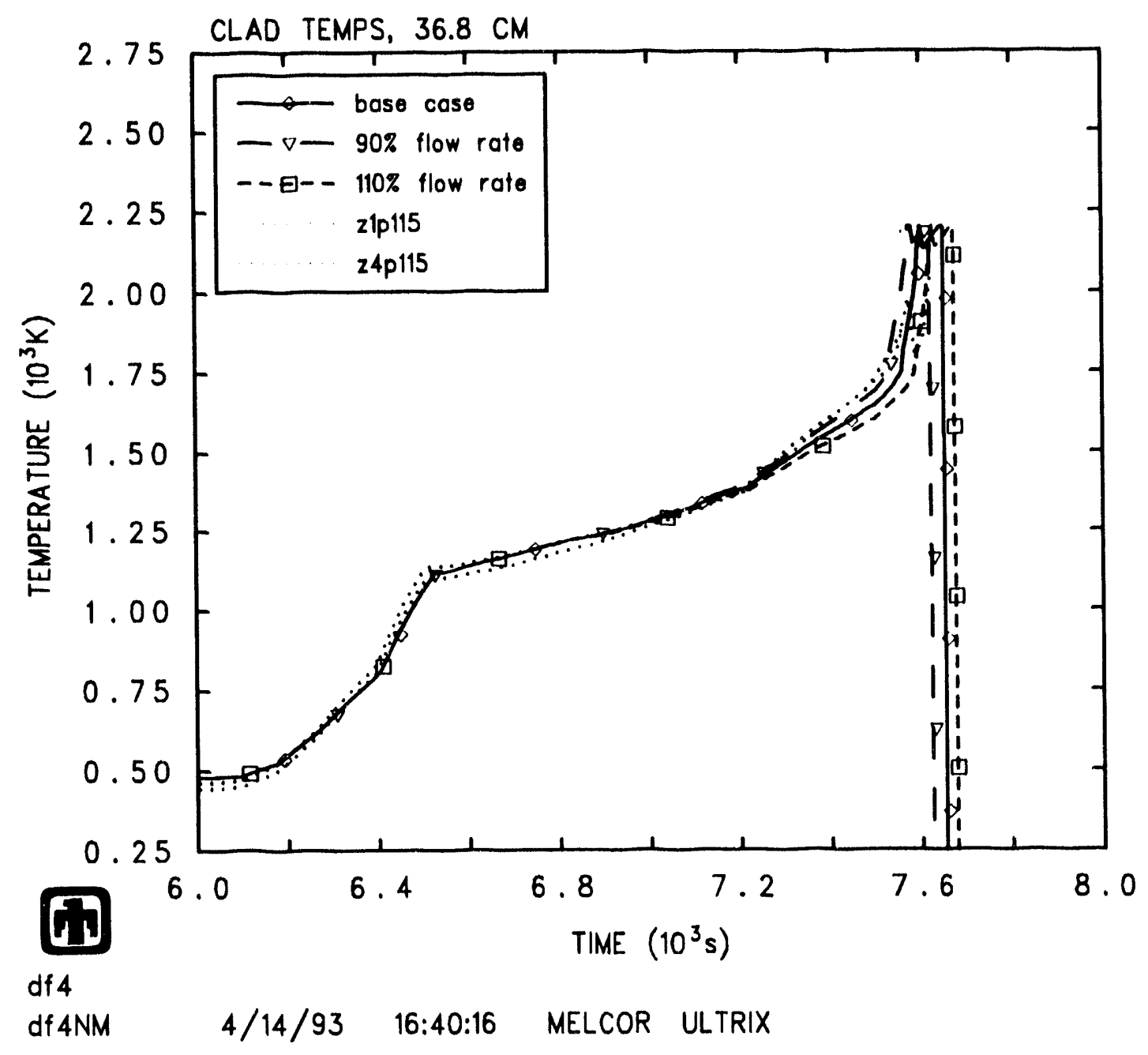

Figure 5.5.1. Cladding temperature response at the $36.8 \mathrm{~cm}$ plane for the steam mass flow rate sensitivity study, compared to base case and measured values. 


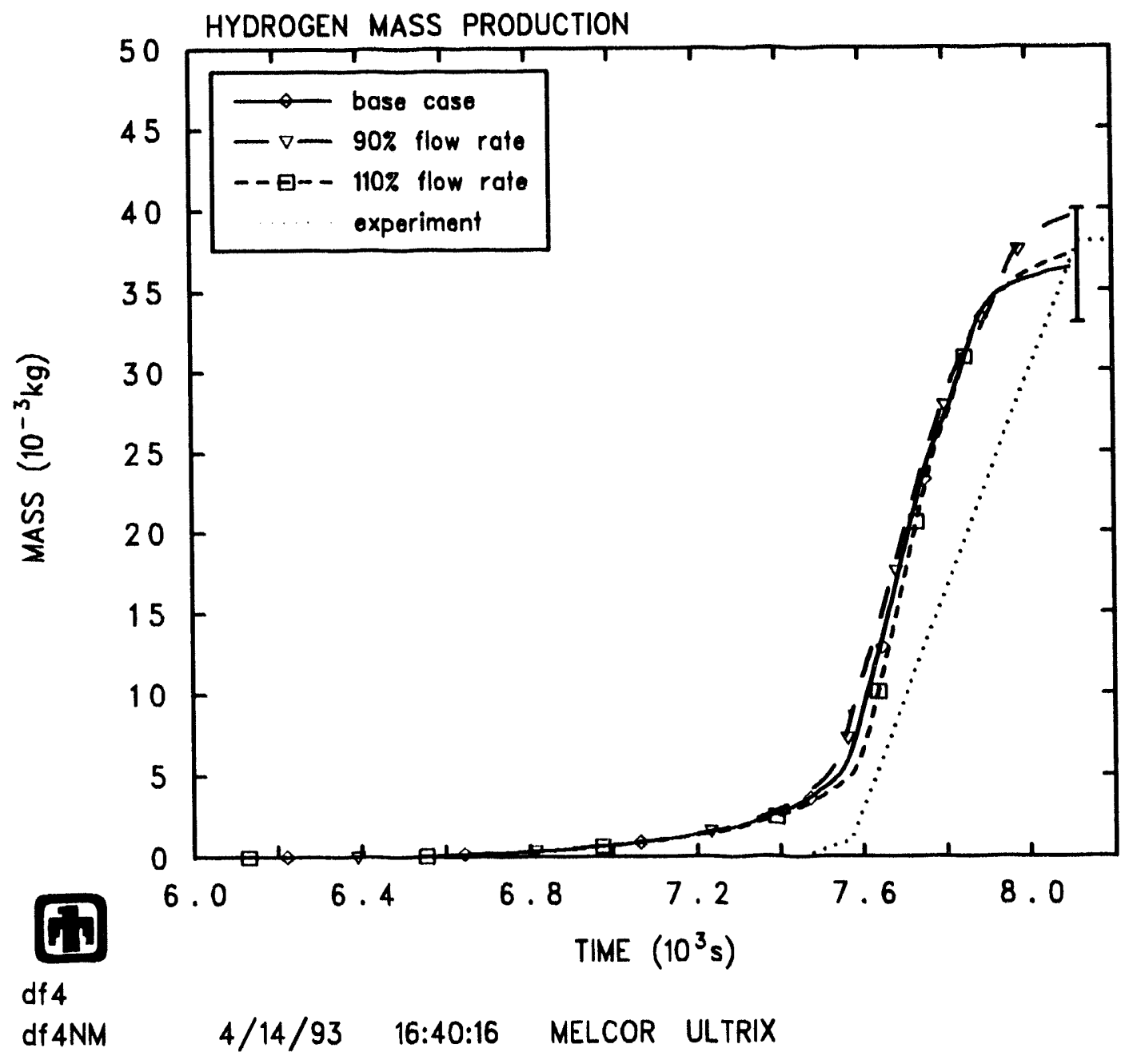

Figure 5.5.2. Hydrogen production for the steam mass flow rate sensitivity study, compared to base case and measured values. 


\subsubsection{Steam Inlet Temperature}

The steam inlet temperature in the experiment was measured using a thermocouple in the inlet plenum below the DF-4 test bundle. This data was provided on a data channel and was used in the MELCOR base case model. For this sensitivity study, the inlet temperature was varied by $\pm 10 \%$. This was done by scaling the control function which specified the temperature in the time-dependent volume below the test bundle in the MELCOR DF-4 model.

The cladding temperature at the $25.4 \mathrm{~cm}$ plane for this study is compared with the base case and measured results in Figure 5.5.3. Increasing and decreasing the steam inlet temperature had the expected results of increasing and decreasing cladding temperatures and causing earlier and later failure, respectively. The variations from the base case were very slight; this was not surprising, considering the overall temperature increase as the steam traveled up the test bundle. The temperature response of the control blade was very similar.

The hydrogen production is shown in Figure 5.5.4. Increasing the inlet temperature resulted in slightly increased hydrogen production early on, but resulted in less overall hydrogen production. This was the result of more zircaloy ending up in debris, which was then covered by a layer of $\mathrm{ZrO}_{2}$ which prevented further oxidation. Decreasing the inlet temperature resulted in the same amount of hydrogen produced as the base case.

In conclusion, the MELCOR results for this experiment were not very sensitive to the steam inlet temperature, due to the large temperature rise in the steam as it traveled up the test bundle.

\subsubsection{Porous Zirconia Thermal Conductivity}

Part of the test bundle insulation consisted of a porous zirconia material whose density was approximately $23 \%$ that of fully dense $\mathrm{Z} \mathrm{rO}_{2}$. A correlation for the thermal conductivity was given in [5] which accounted for the enhanced conductivity due to steam absorbed in this material. This correlation is given in Equation (3.1). For this study, the thermal conductivity was varied by $\pm 25 \%$ by scaling the control function used to input this correlation.

The cladding temperatures at the $36.8 \mathrm{~cm}$ plane for this study are compared with base case and experimental results in Figure 5.5.5. The results changed only at higher temperatures, when radiation heat transfer to the boundary heat structure and heat conduction through that structure were significant. Increasing and decreasing the conductivity through the heat structure resulted in decreasing and increasing the energy absorbed in the test bundle components, respectively. The more energy absorbed in the core components, the earlier component failure occurred. Temperature behaviors at other levels and in the control blade were very similar.

The hydrogen produced for this case is compared to the base case and measured results in Figure 5.5.6. The timing of hydrogen production was affected by changes to the heat structure conductivity, but the total amount of hydrogen produced was changed only for the case which increased the heat structure conductivity. The de- 


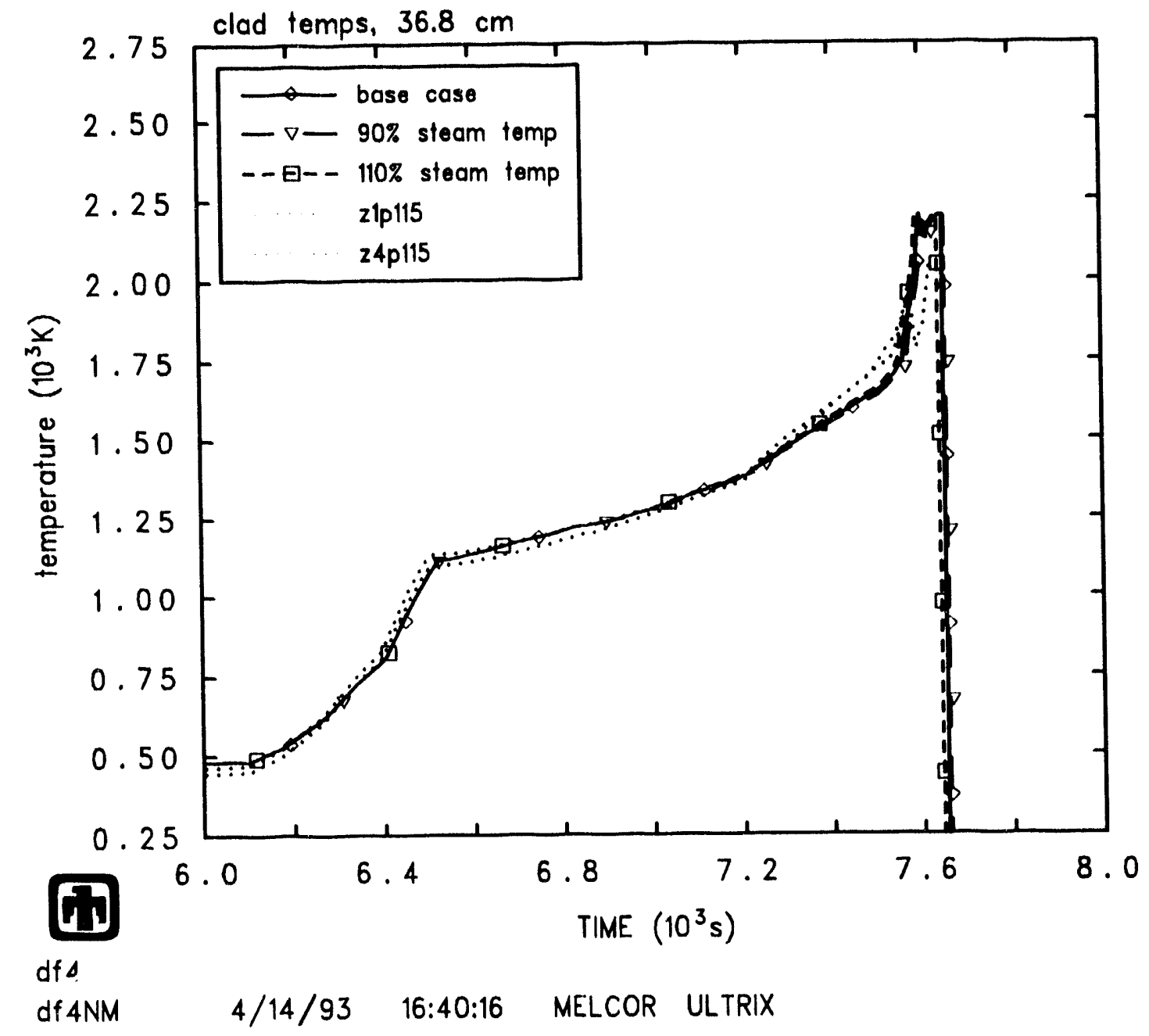

Figure 5.5.3. Cladding temperature response at the $36.8 \mathrm{~cm}$ plane for the steam inlet temperature sensitivity study, compared to base case and measured

values. 


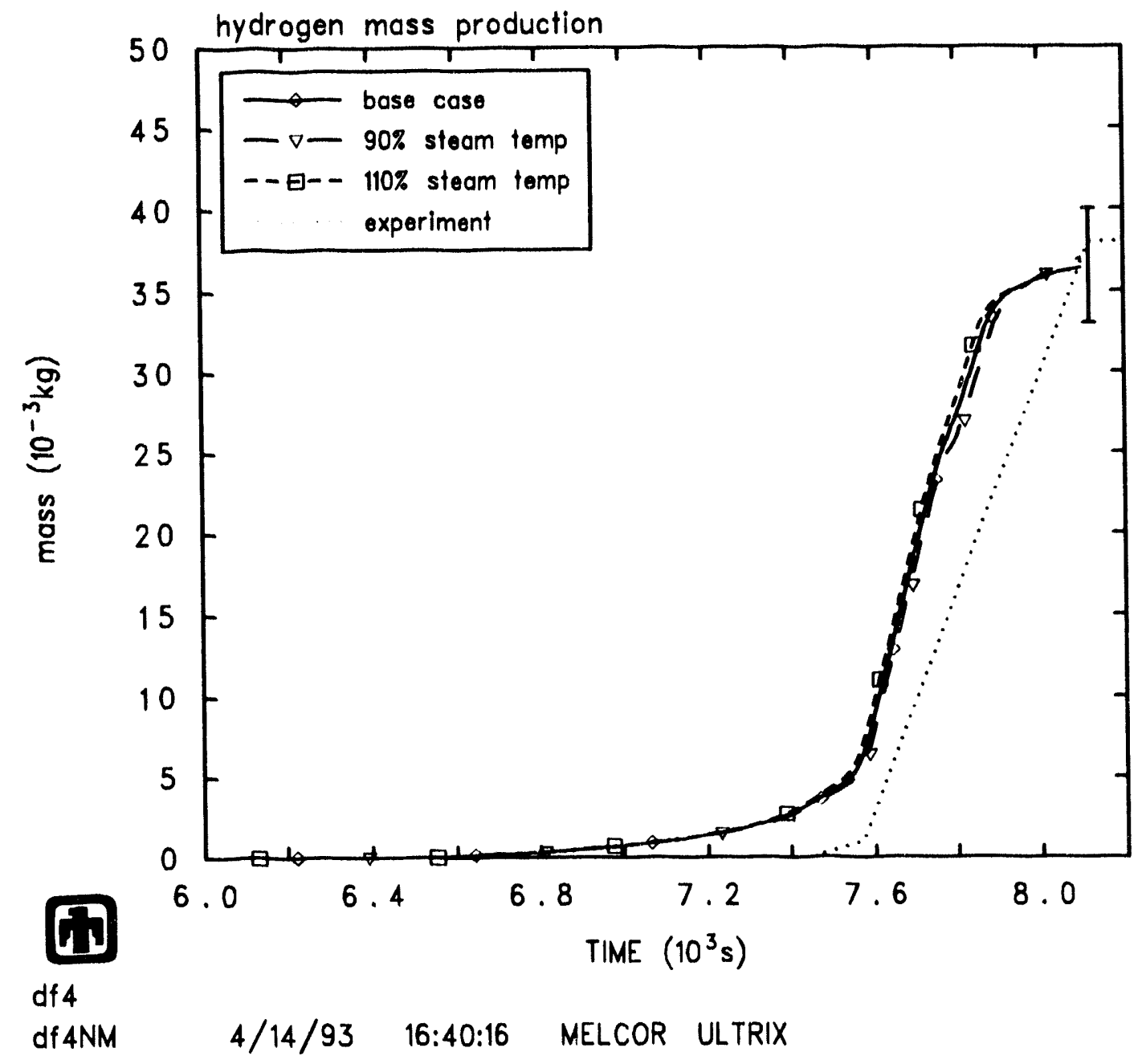

Figure 5.5.4. Hydrogen production for the steam inlet temperature sensitivity study, compared to base case and measured values. 
crease in hydrogen production was probably due to the increased heat loss through the heat structure.

The MELCOR results were sensitive to the boundary heat struclure conductivity. This was not surprising, given the high exposure of the fuel rods to the insulating boundary.

\subsubsection{Radial Heat Structures Outer Boundary Temperature}

The outer surface of the boundary heat structure in the MELCOR base case model corresponded to the pressure boundary shown in Figure 2.1.1. In the experiment, this boundary was heated to $473 \mathrm{~K}$ to prevent condensation on the inside surface of the test bundle insulation. 'The outer surface of the boundary heat structure was held at this temperature in the MELCOR base case model. During the experiment, this surface was actually heated to higher temperatures because of heat transfer from the test bundle region. In this study, the temperature of this surface was raised and lowered by $10 \%$ (about $50 \mathrm{~K}$ ). In addition, a temperature ramp case was performed, where the temperature was raised from $460 \mathrm{~K}$ to $600 \mathrm{~K}$ on an even ramp from 6000 to 8500 seconds. This was accomplished using a control function to specify the outer boundary temperature for all radial heat structures in the MELCOR base case model.

The temperature and hydrogen production results for this study are compared to base case and measured results in Figures 5.5.7 and 5.5.8. These figures show that the MELCOR base case calculation for DF-4 was not very sensitive to changes in the outer boundary temperature of the radial heat structures.

\subsubsection{Bundle Fission Power}

The fission power experienced by the test bundle components was input to the MELCOR base case modei using a power coupling correlation from [5]. This correlation was derived from ACRR power increases and measured cladding temperature response during the initial nuclear heating subphase of the experiment. Figure 3.2 .2 shows that there were large uncertainties (between 10\% and $40 \%$ ) in the coupling between the ACRR and the test bundle fission powers. In this study, the total amount of test bundle power was adjusted by $\pm 10 \%$, using the same peaking factors derived from Equation (3.2). This was accomplished by scaling the control function used to input total fission power.

Figures 5.5.9 and 5.5.10 show that tie MELCOR base case calculation for DF4 was quite sensitive to the amount of fission power deposited in the test bundle. The deviations in temperature, failure timing and total hydrogen production were all nearly proportional to bundle power changes. Temperatures in other axial levels and components were very similar. 


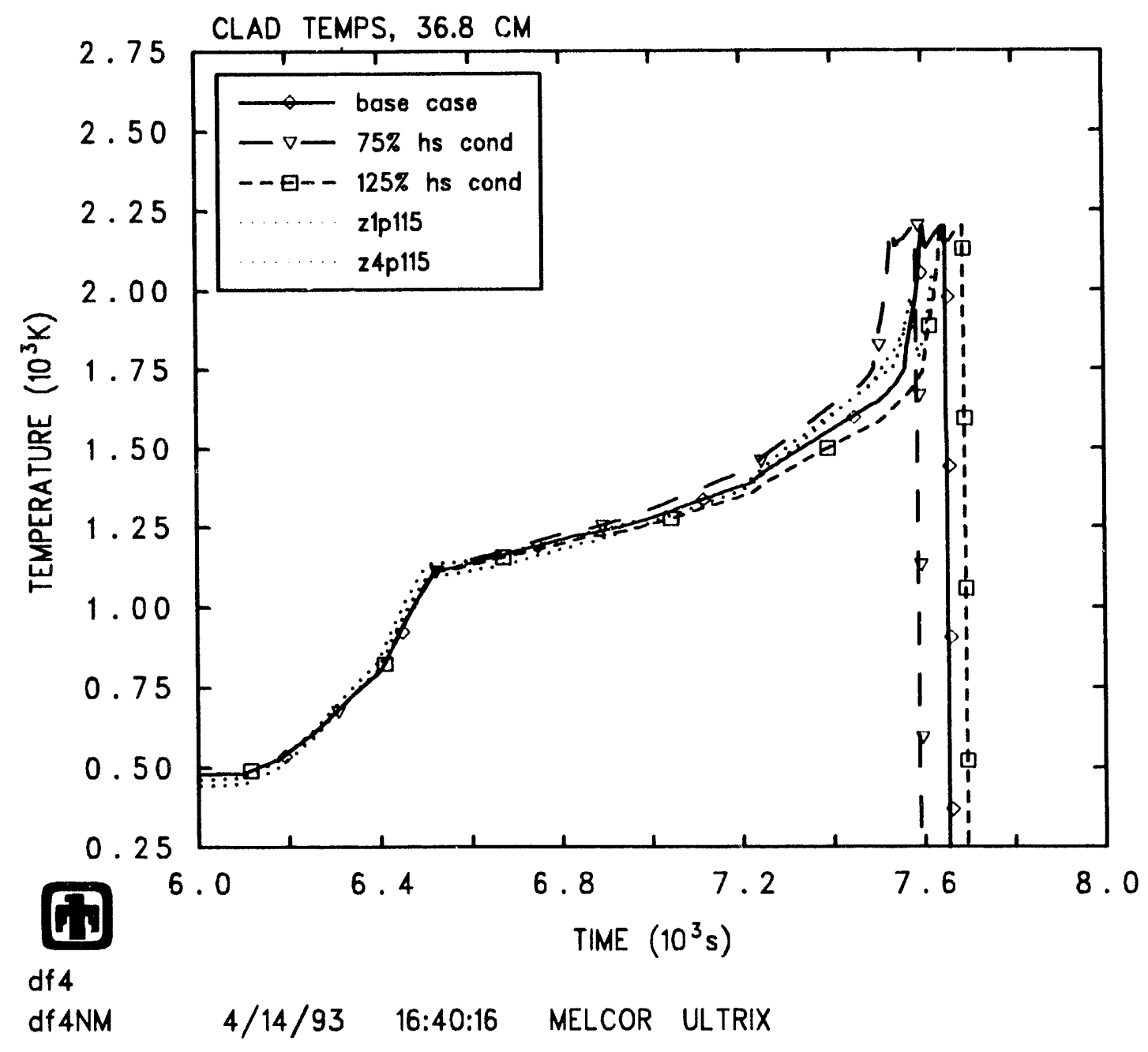

Figure 5.5.5. Cladding temperature response at the $36.8 \mathrm{~cm}$ plane for the boundary heat structure conductivity sensitivity study, compared to base case and measured values. 


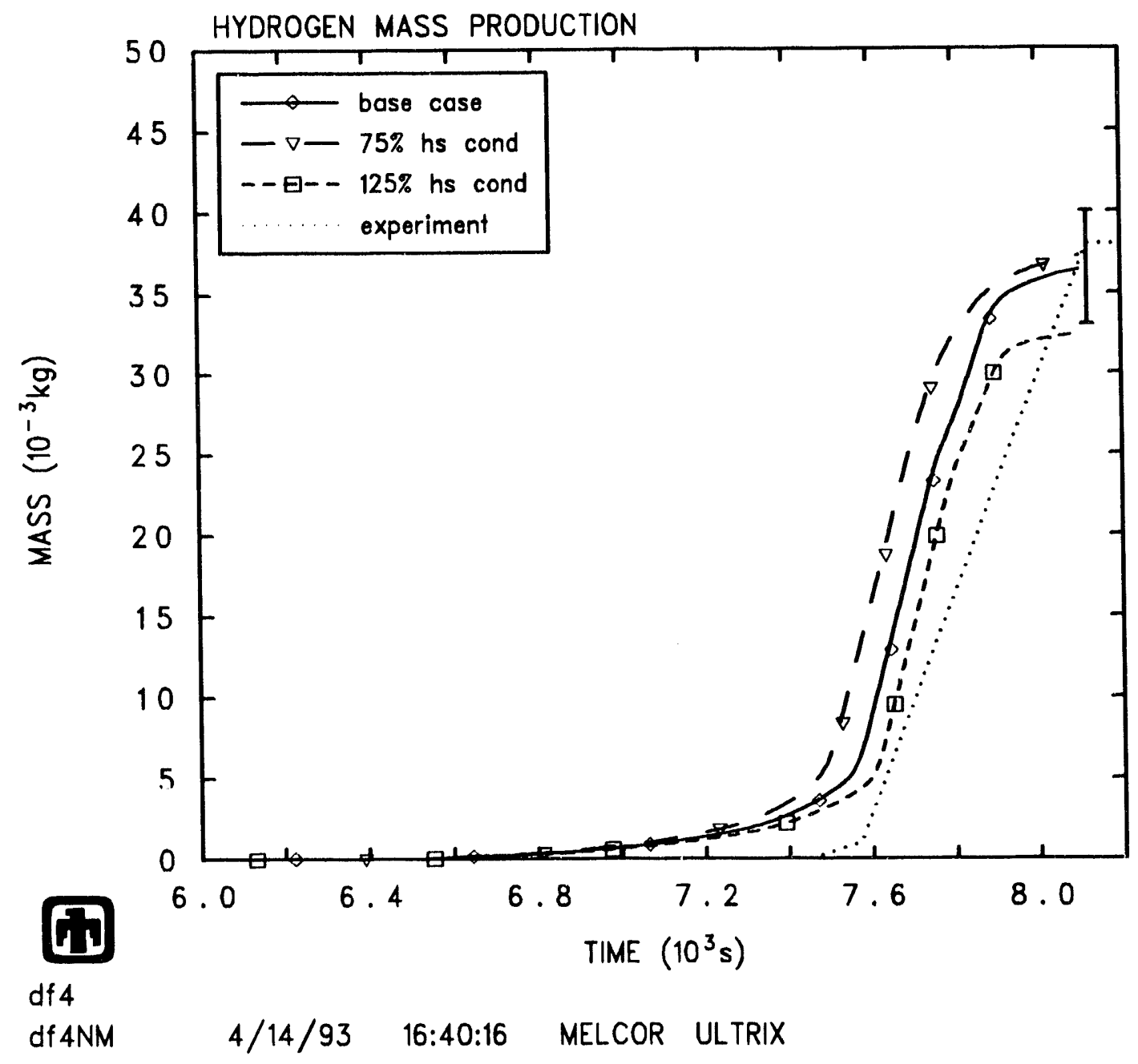

Figure 5.5.6. Hydrogen production for the boundary heat structure conductivity sensitivity study, compared to base case and measured values. 


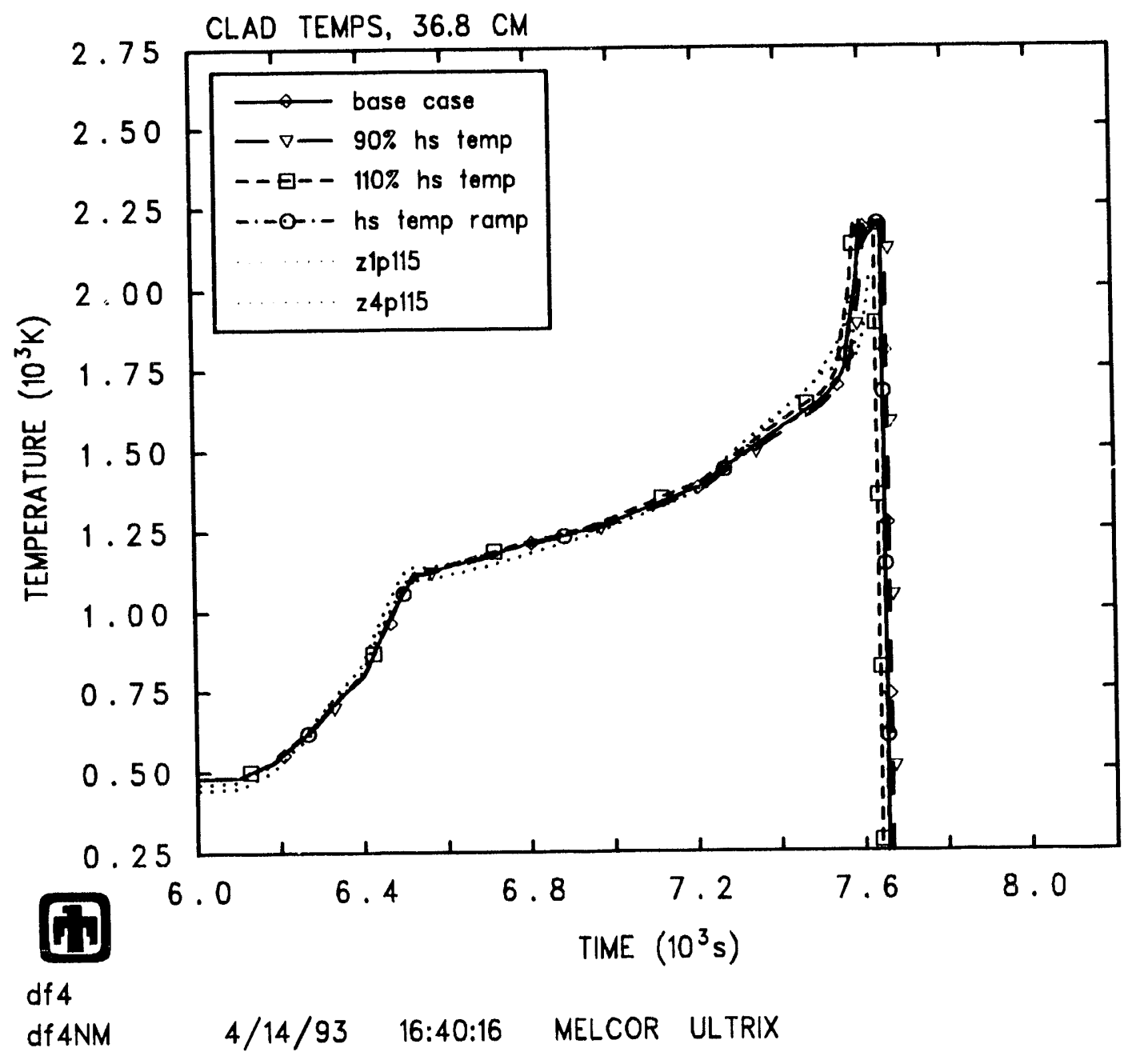

Figure 5.5.7. Cladding temperature response at the $36.8 \mathrm{~cm}$ plane for the boundary heat structure temperature sensitivity study, compared to base case and measured values. 


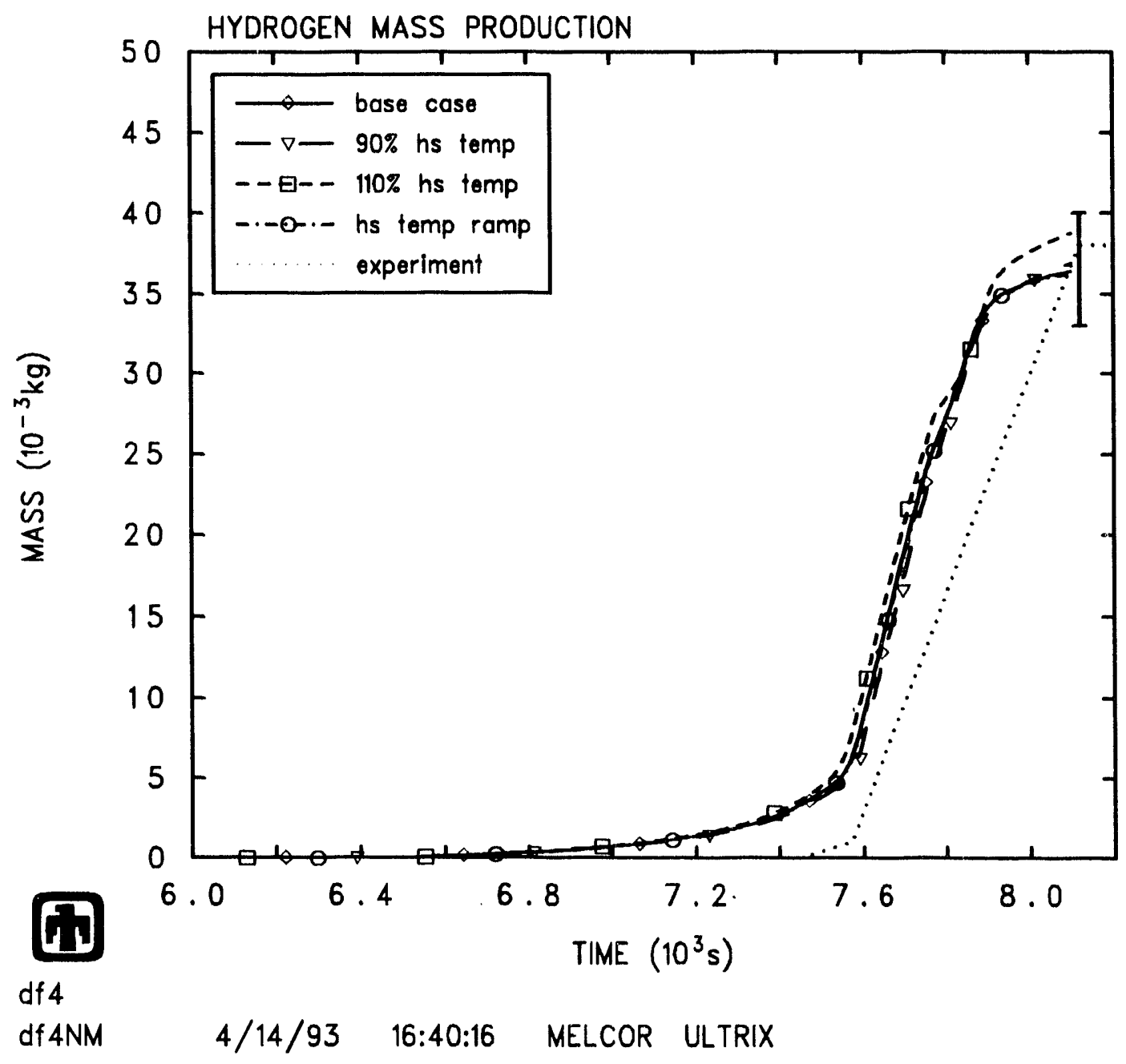

Figure 5.5.8. Hydrogen production for the boundary heat structure temperature sensitivity study, compared to base case and measured values. 


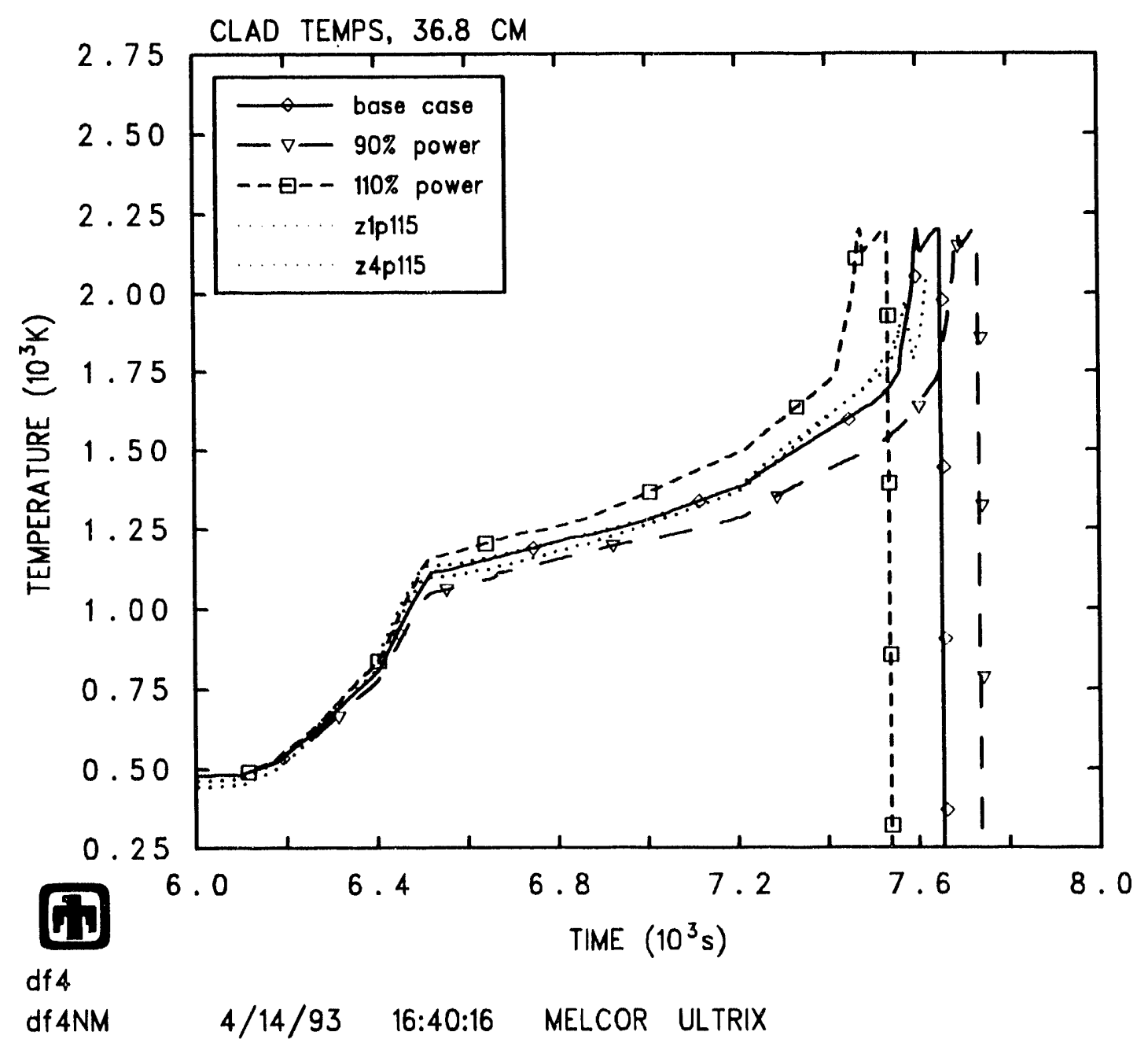

Figure 5.5.9. Cladding temperature response at the $36.8 \mathrm{~cm}$ plane for the bundle power sensitivity study, compared to base case and measured values. 


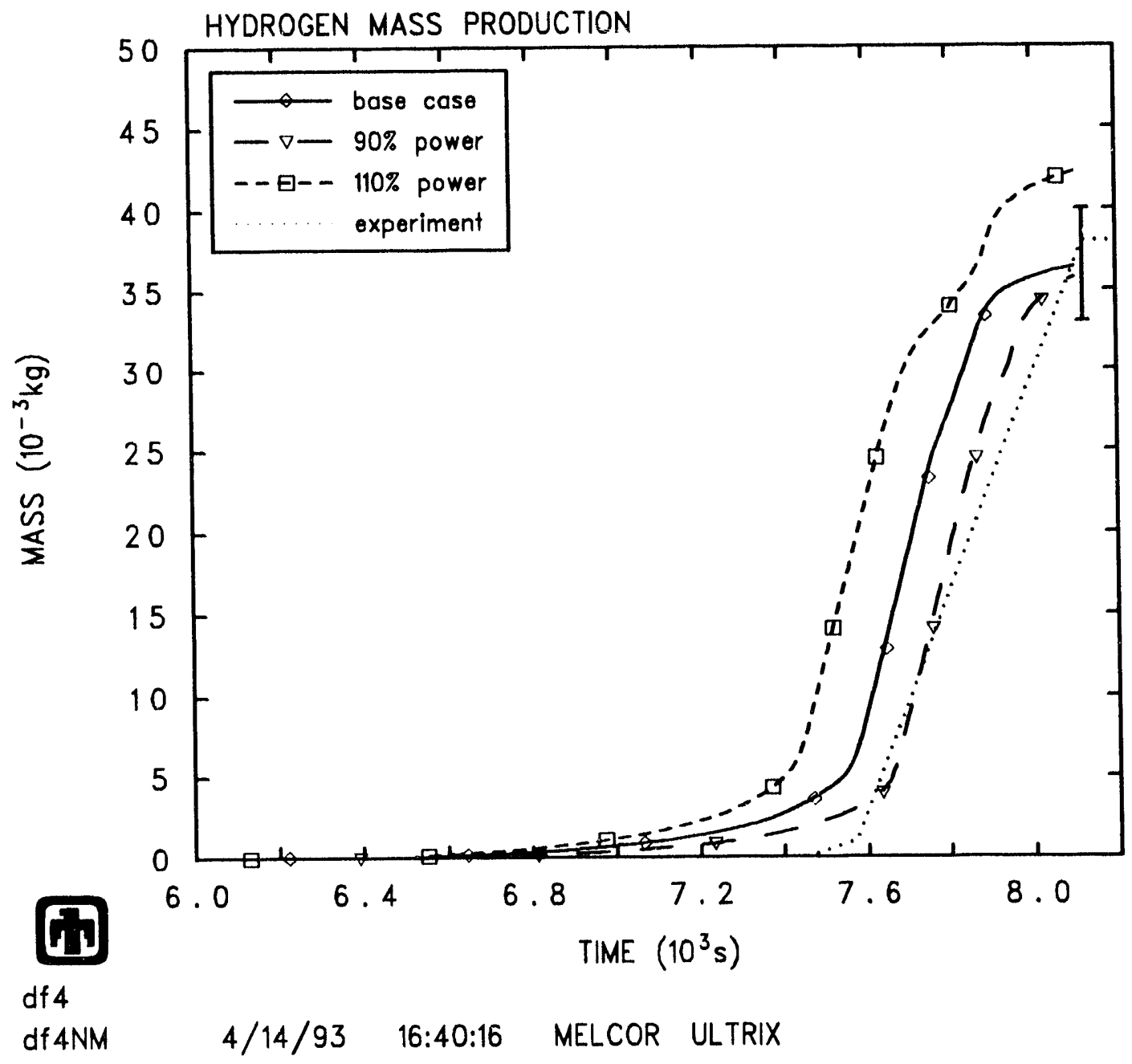

Figure 5.5.10. Hydrogen production for the bundle power sensitivity study, compared to base case and measured values. 


\subsection{General Heat Structures Package Studies}

Two sensitivity studies were done on HS package parameters. The first was on the radiation model used for all heat structures, and the second was on the emissivities used for all heat structures.

\subsubsection{HS Radiation Model Option}

The heat structures package has two options for calculating radiation heat transfer from the steam environment to the heat structures. The base case model used the default option, the equivalent band ("equiv-band") model. In this study, the model was changed to use the "gray-gas-a" option.

The temperature and hydrogen production results from this study were almost identical to the base case results. The radiation model employed in the heat structures package had no effect on the final calculated results for this problem.

\subsubsection{Heat Structures Emissivities}

The emissivities for all heat structures surfaces in the MELCOR base case models were chosen arbitrarily to be 0.7 . A sensitivity study was done to investigate the effects of varying this emissivity. Two cases were done for this study, setting the emissivities for all heat structures surfaces to 0.5 and 0.9 .

The temperature and hydrogen production results for both cases of this study were almost identical to those of the MELCOR base case. Thus, there was very little sensitivity to the emissivity used for heat structures surfaces.

\subsection{COR and CVH Nodalization}

In the MELCOR base case model, there were nine core cells in the active fuel region and two control volumes in the core (one each for the channel and bypass regions). Four variations on the nodalization in the DF-4 model were performed for this study. For the first three cases, the number of core cells in the active fuel region was changed to 5,18 and 27 core cells. For the last case, the 18 cell problem was used with six CVH control volumes instead of two, where the active fuel region was split equally among three axial control volumes in the channel and bypass regions.

The cladding and control blade temperatures at the $36.8 \mathrm{~cm}$ plane are shown in Figures 5.7.1 and 5.7.2, respectively. For the first 500 seconds of the problem, the temperature response was very close in all cases. The temperatures diverge after that, and generally the cases with the finer nodalizations calculated higher cladding temperatures and early failure times. Note that the temperatures monotonically increase with the number of core cells used, with the base case results closer to the finer nodalization cases.

The hydrogen production for all cases is compared to base case and measured results in Figure 5.7.3. Like the temperature response, the hydrogen response is 
sensitive to nodalization, with the two finest nodalization problems producing less hydrogen and the other problems producing about as much hydrogen as the base case calculation. Note that only the 27 -cell problem hydrogen production is outside the uncertainty band of the PIE data.

The reason for higher temperatures and lower hydrogen production with finer nodalizations can possibly be linked to the competition between advection and oxidation for steam flowing through the test bundle. The increased temperatures could be caused by the same effect, where smaller core cells have less steam cooling (this is only a suggestion; it could also be argued that smaller core cells produce less heat and therefore require less cooling). This study shows the need for better time step control during periods of rapid zircaloy oxidation.

\subsection{Time Step and Machine Dependency Studies}

The MELCOR code contains both global time step control and package-specific time step subcycling. However, the effectiveness of that time step control is sometimes suspect, and it is always a good idea to investigate a range of time steps for any particular input model. Also, MHICCOR is considered a very portable code and does in fact run on many different computer platforms of both 32-bit and 64-bit architectures. Comparing code results across these platforms can reveal both machine dependencies and general coding problems [10]. In this section, studies that were performed to investigate the time step control in MELCOR and the dependency of DF-4 results on computer platform are discussed.

\subsubsection{Time Step Study}

The time step in the 1$) \mathrm{F}-4$ base case model was restricted to a maximum of 0.25 seconds between 7200 and 8000 seconds. This was the period of time when core degradation took place. During the rest of the calculation, the maximum time step was set to 1.0 seconds. For this sensitivity study, cases were run with the maximum time step set at 0.1 seconds, 0.5 seconds, 1.0 second and a time step much larger than the MELCOR-default time step between 7200 and 8000 seconds (the maximum time step for the final case: was chosen such that the code always used the MELCOR-chosen time step).

The overall MELCOR time step used by the four cases is compared with that used by the base case model in Figure 5.8.1. The overall code was using the specified maximum time step, except for the case that used the MELCOR-default time step. The COR package time step used by the four cases is compared with that used by the base case model in Figure 5.8 .2 . For time steps of 0.1 and 0.25 seconds, the COR package time step is the same as the system time step. However, using a time step) above 0.25 seconds caused the (OR package to choose smaller time steps. This caused difficulties when core components began to oxidize. The cladding temperature at the $49.5 \mathrm{~cm}$ plane in Figure 5.8 .3 shows the problem encountered in DF-4 runs using timesteps that were too large. At approximately 7400 seconds, the " $\Delta t=$ default" 


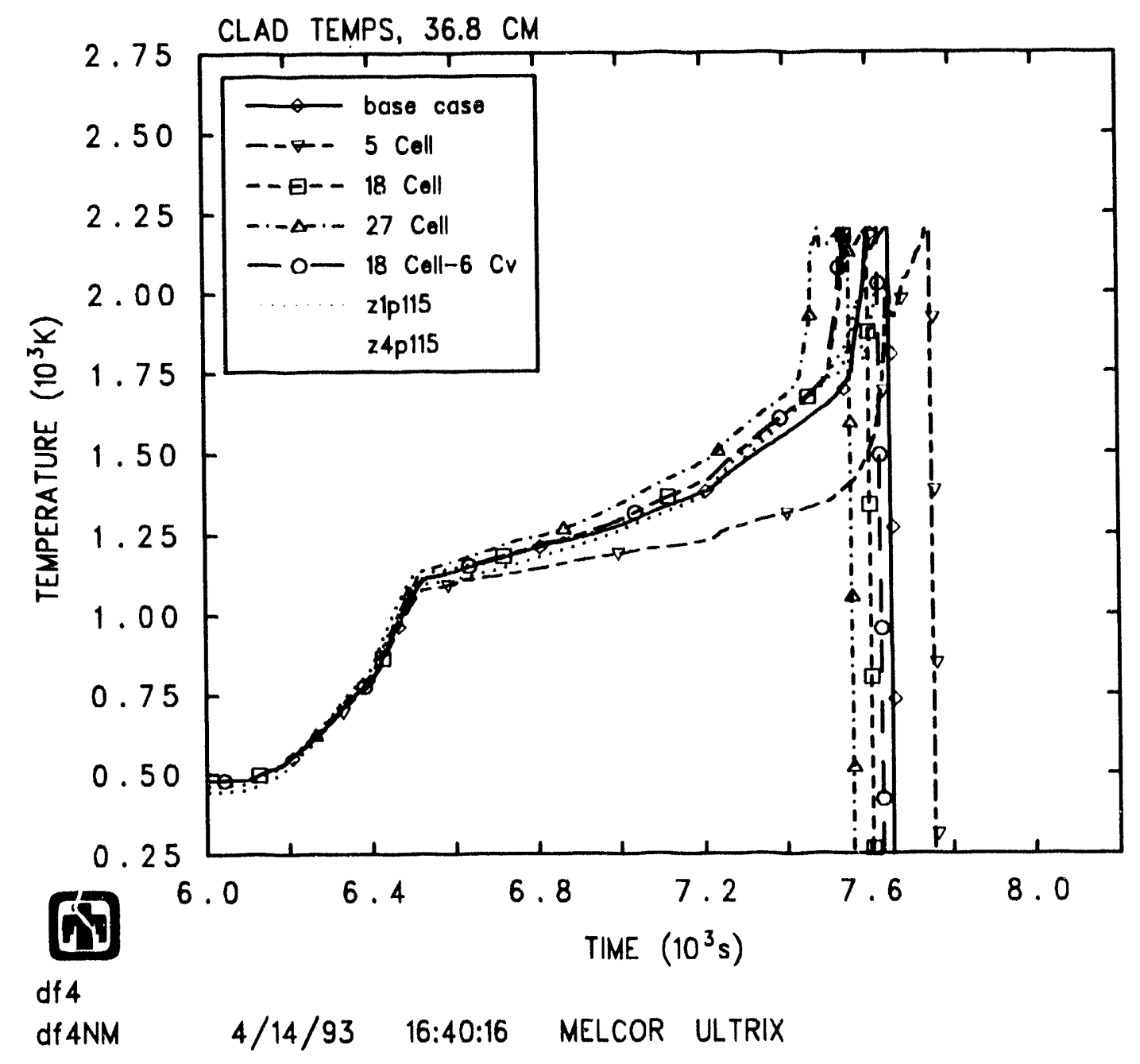

Figure 5.7.1. Cladding temperature response at the $36.8 \mathrm{~cm}$ plane for the $\mathrm{COR}$ and CVH nodalization sensitivity study, compared to base case and measured values. 


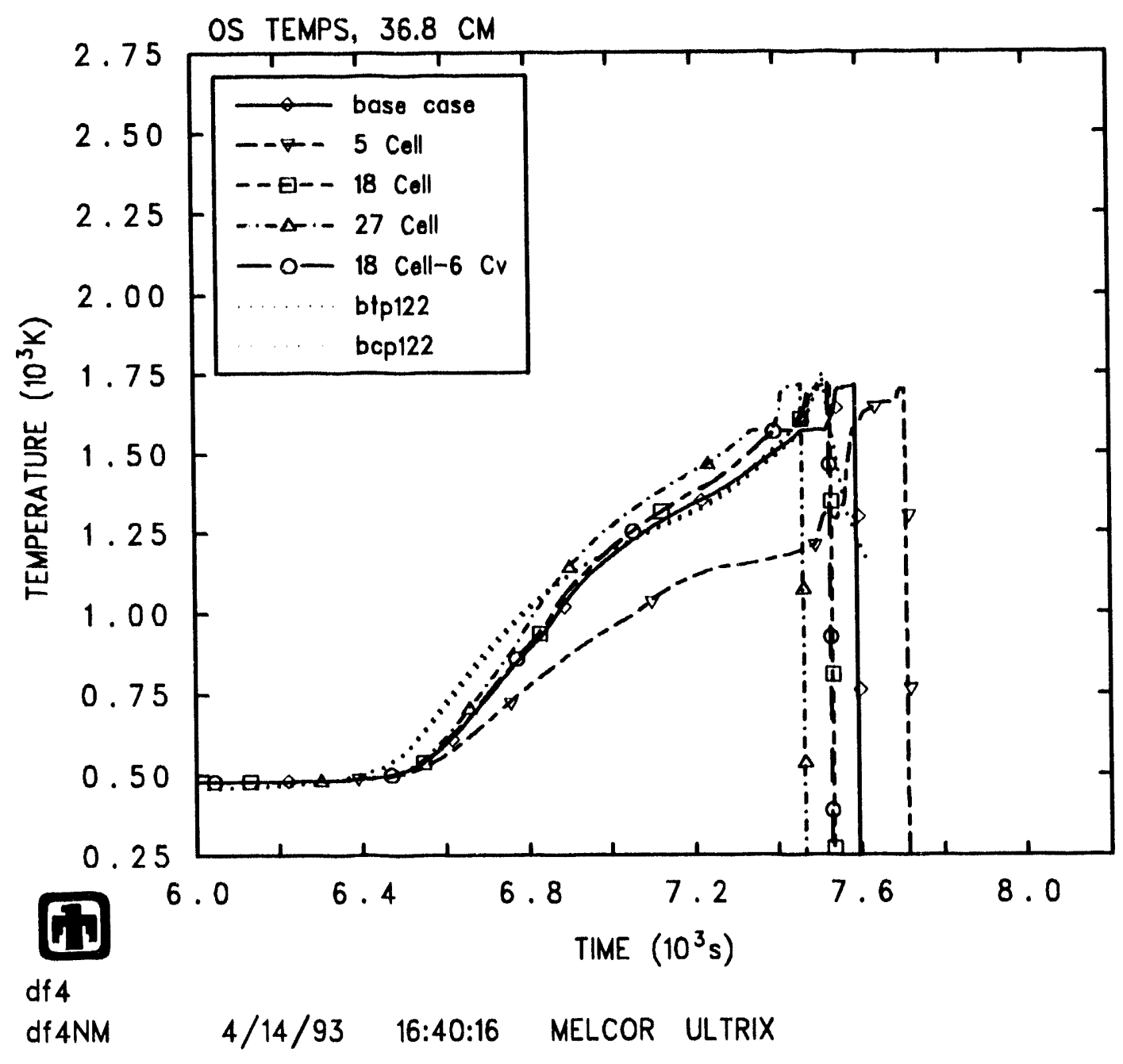

Figure 5.7.2. Control blade temperature response at the $36.8 \mathrm{~cm}$ plane for the $\mathrm{COR}$ and $\mathrm{C}, \mathrm{VH}$ nodalization sensitivity study, compared to base case and measured values. 


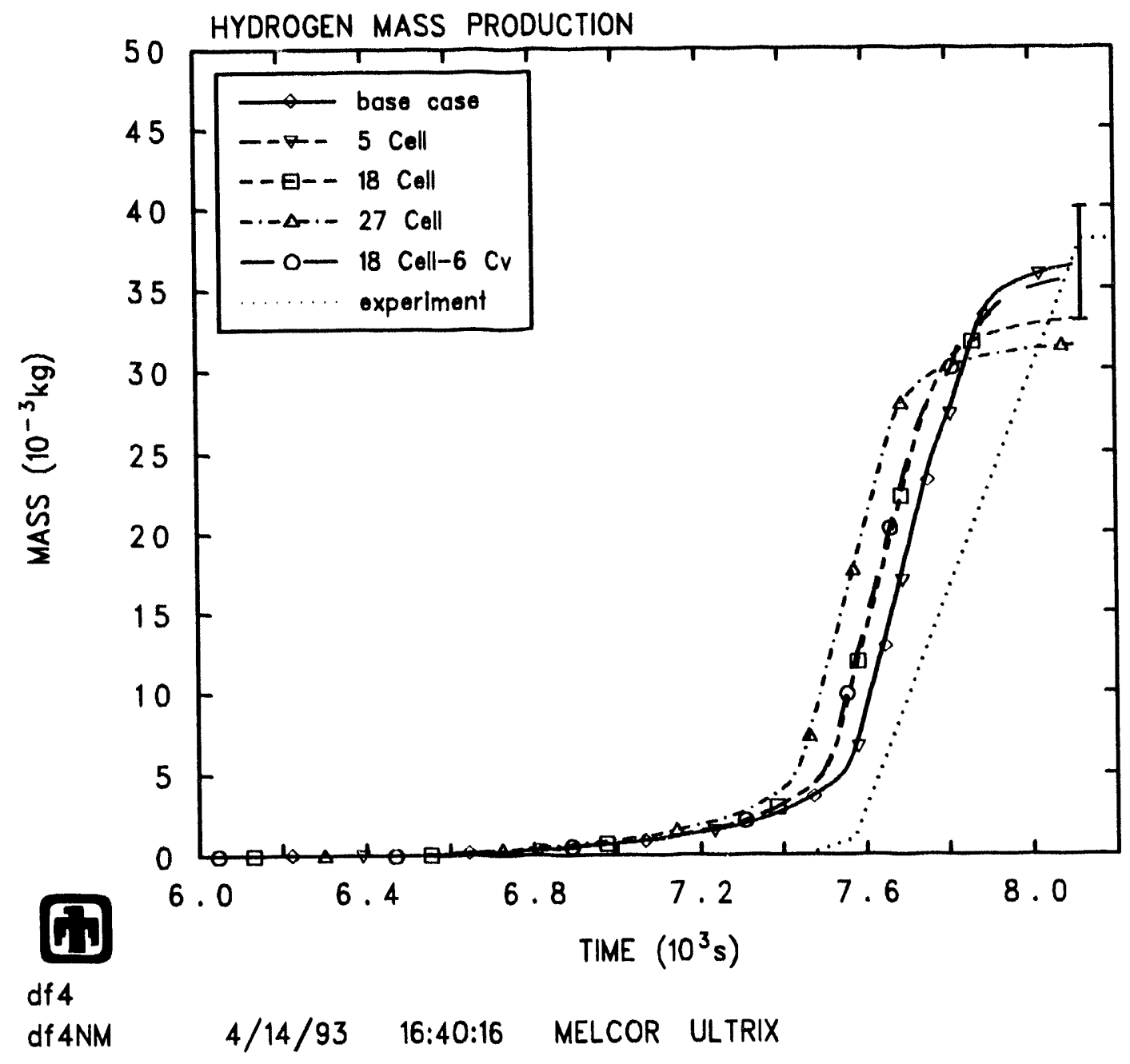

Figure 5.7.3. Hydrogen production for the bundle power sensitivity study, compared to base case and measured values. 
case showed a spike in temperature. The cause of this was apparently the beginning of oxidation at that axial level, which stopped soon afterward due to steam starvation. This phenomenon went away when a smaller time step was used. This was the reason for limiting the time step to 0.25 seconds between 7200 and 8000 seconds for the base case calculation.

The cladding and control blade temperatures at the $36.8 \mathrm{~cm}$ and $25.4 \mathrm{~cm}$ planes are shown in Figures 5.8.4 and 5.8.5, respectively. Temperature behavior before 7200 seconds was identical because the same time step was being used in all cases. After 7200 seonds, results were also relatively close, until the beginning of rapid oxidation. There was a spread of approximately 100 seconds around all cases for the start of rapid oxidation in the cladding at the $36.8 \mathrm{~cm}$ plane. Also, for this plane the base case temperature data was bounded by other cases. The spread in failure time of the cladding at this plane was less than 100 seconds. The temperatures in the control blade were also very close for all cases, until the beginning of the $\mathrm{B}_{4} \mathrm{C}$-stainless steel eutectic interaction. After this point, the temperatures drifted apart, but were still relatively close. The spread of failure times was approximately 100 seconds, similar to the spread in cladding failure times at the $36.8 \mathrm{~cm}$ plane.

The hydrogen production for the time step study cases is shown in Figure 5.8.6, compared to base case and measured results. The hydrogen production in the base case model was bounded by that of the cases run for this study. It is difficult to find the reason for variations in hydrogen production with differing time steps, because of competing time step-dependent phenomena affecting hydrogen production (e.g., core material relocation and steam starvation effects). In any event, a sensitivity study should always be done on time steps used in MELCOR, at least for the portions of a calculation when core materials are degrading or oxidizing rapidly.

In conclusion, this study showed that core component temperatures calculated by MELCOR for this problem were slightly sensitive to the choice of time step, and that hydrogen production was somewhat sensitive to time step. The proper time step for this problem was in the neighborhood of $0.1-0.5$ seconds, and was limited by relocation and oxidation phenomena in the COR package. Time steps for other problems will be different since they depend on flow velocity and other accident progression phenomena.

\subsubsection{Machine Dependency Study}

MELCOR 1.8.2 was installed on six different computer platforms, where the DF4 base case input model was run. These computer platforms were: DECstation 5000/240, Hewlett Packard 755, IBM RS/6000 Model 550, SUN Sparcstation 2, Cray $\mathrm{X} / \mathrm{MP}-24$, and Gateway 486/DX2-50. The first four machines are 32-bit UNIX workstations, the fifth a 64-bit supercomputer, and the sixth an IBM PC compatible. The base case results for each of these machines were translated to one machine and cross-plotted to compare results. Note that the base case and ot her sensitivity study calculations were performed on the DECstation 5000/240.

The cladding and control blade temperatures at the $36.8 \mathrm{~cm}$ plane for all machines are compared to measured results in Figures 5.8.7 and 5.8.8, respectively. The 


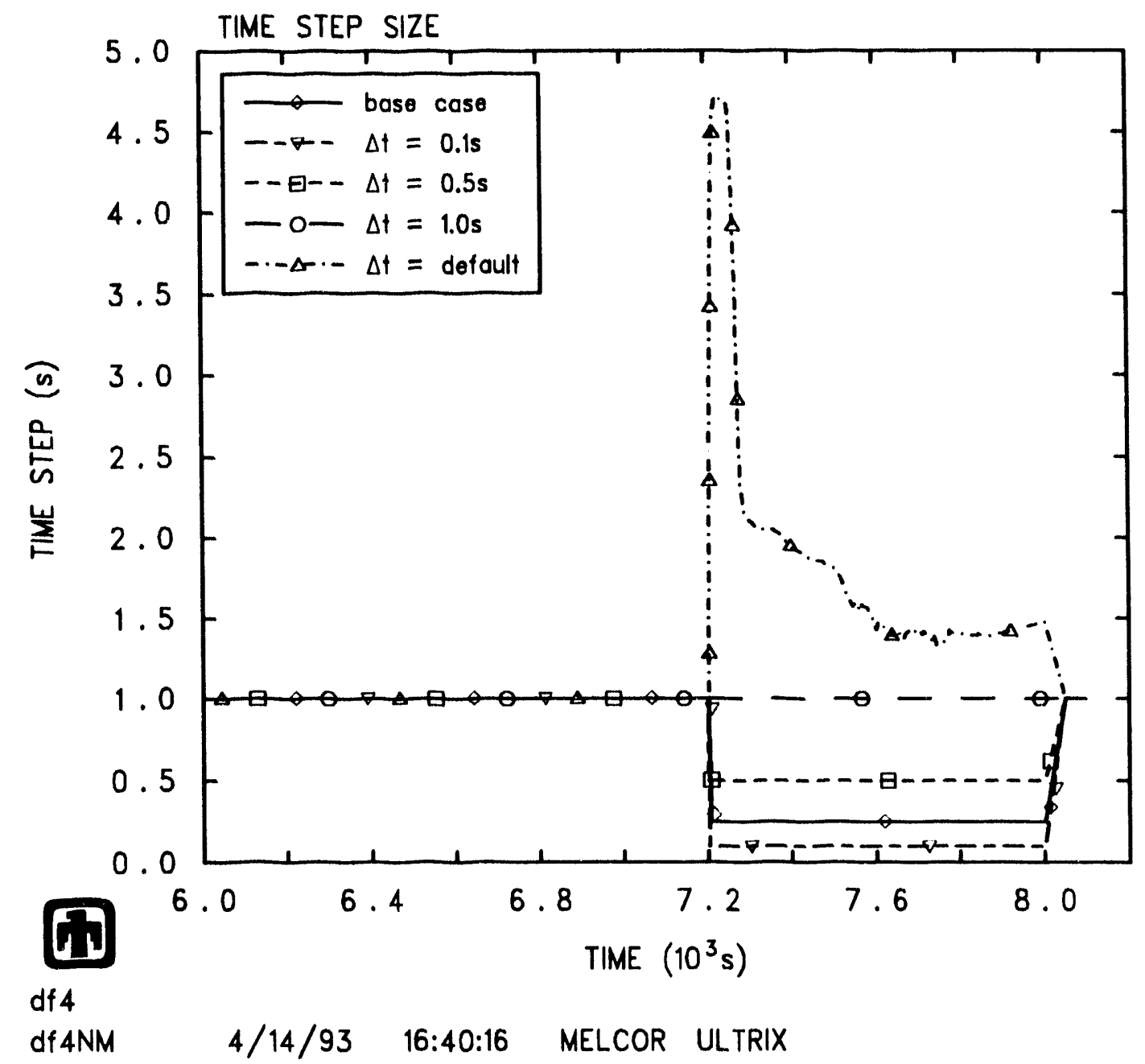

Figure 5.8.1. Time steps used for the time step sensitivity study, compared to base case and measured values. 


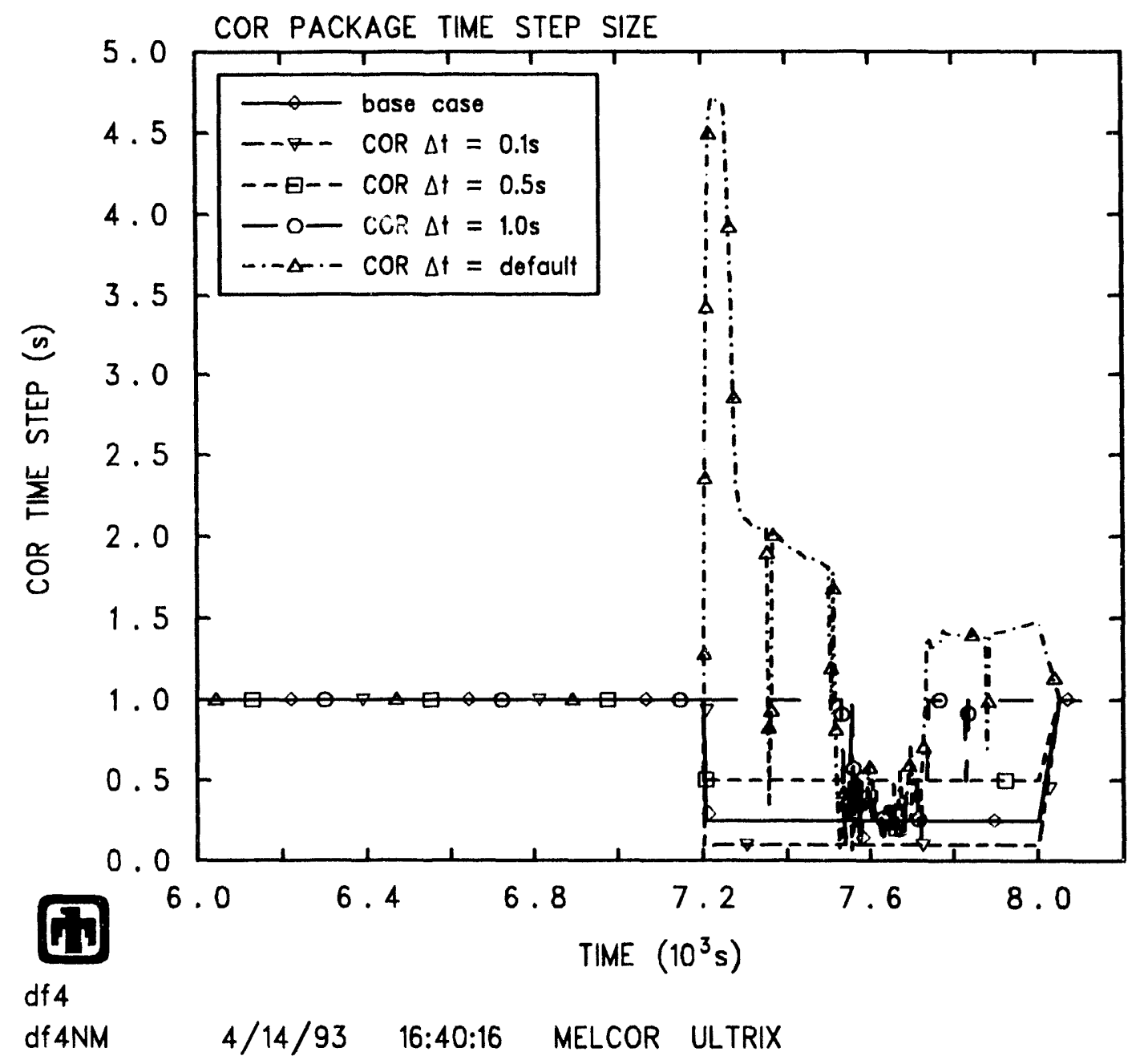

Figure 5.8.2. COR package time steps used for the time step sensitivity study, compared to base case and measured values. 


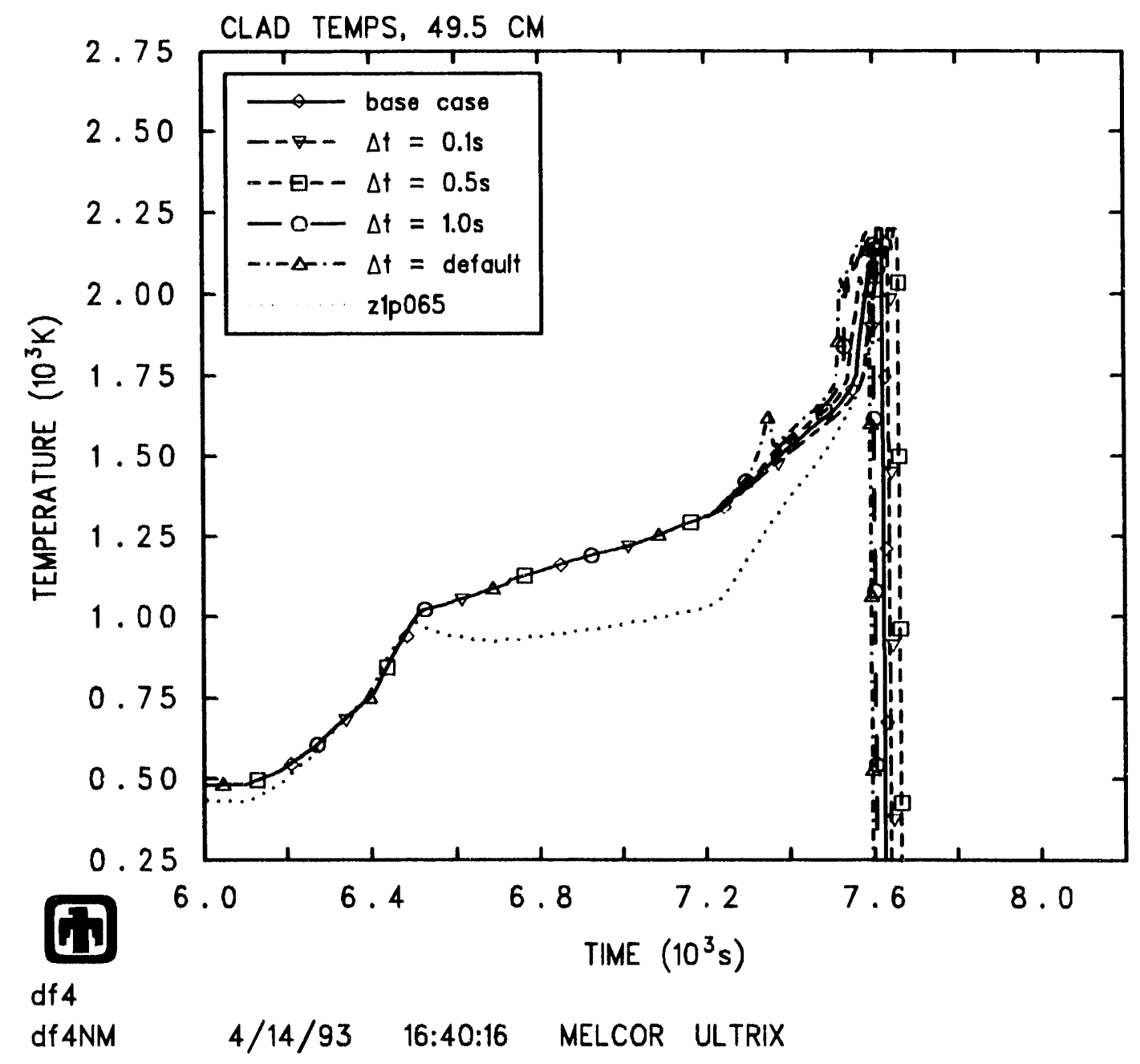

Figure 5.8.3. Cladding temperature response at the $49.5 \mathrm{~cm}$ plane for the time step sensitivity study, compared to base case and measured values. 


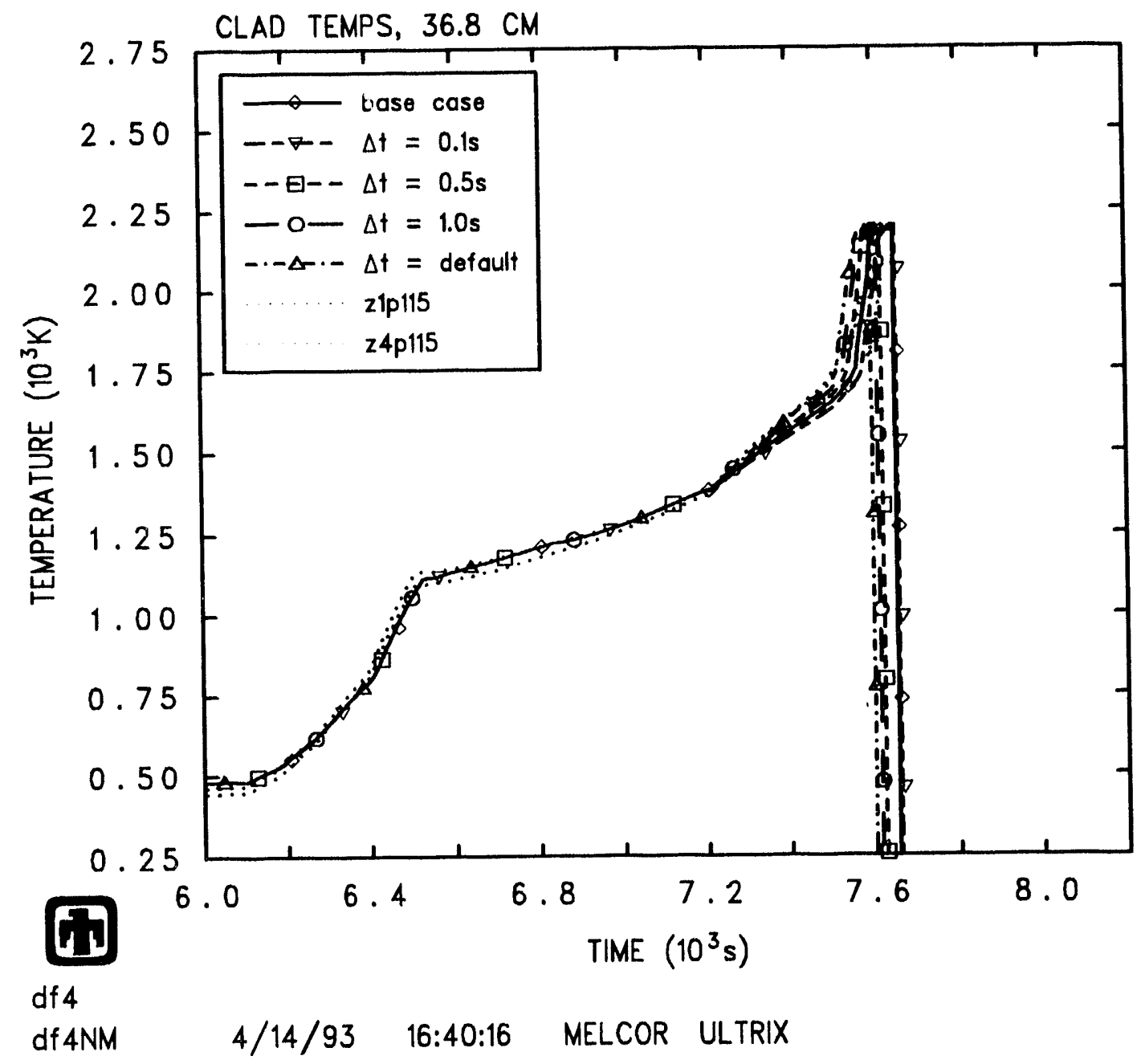

Figure 5.8.4. Cladding temperature response at the $36.8 \mathrm{~cm}$ plane for the time step sensitivity study, compared to base case and measured values. 


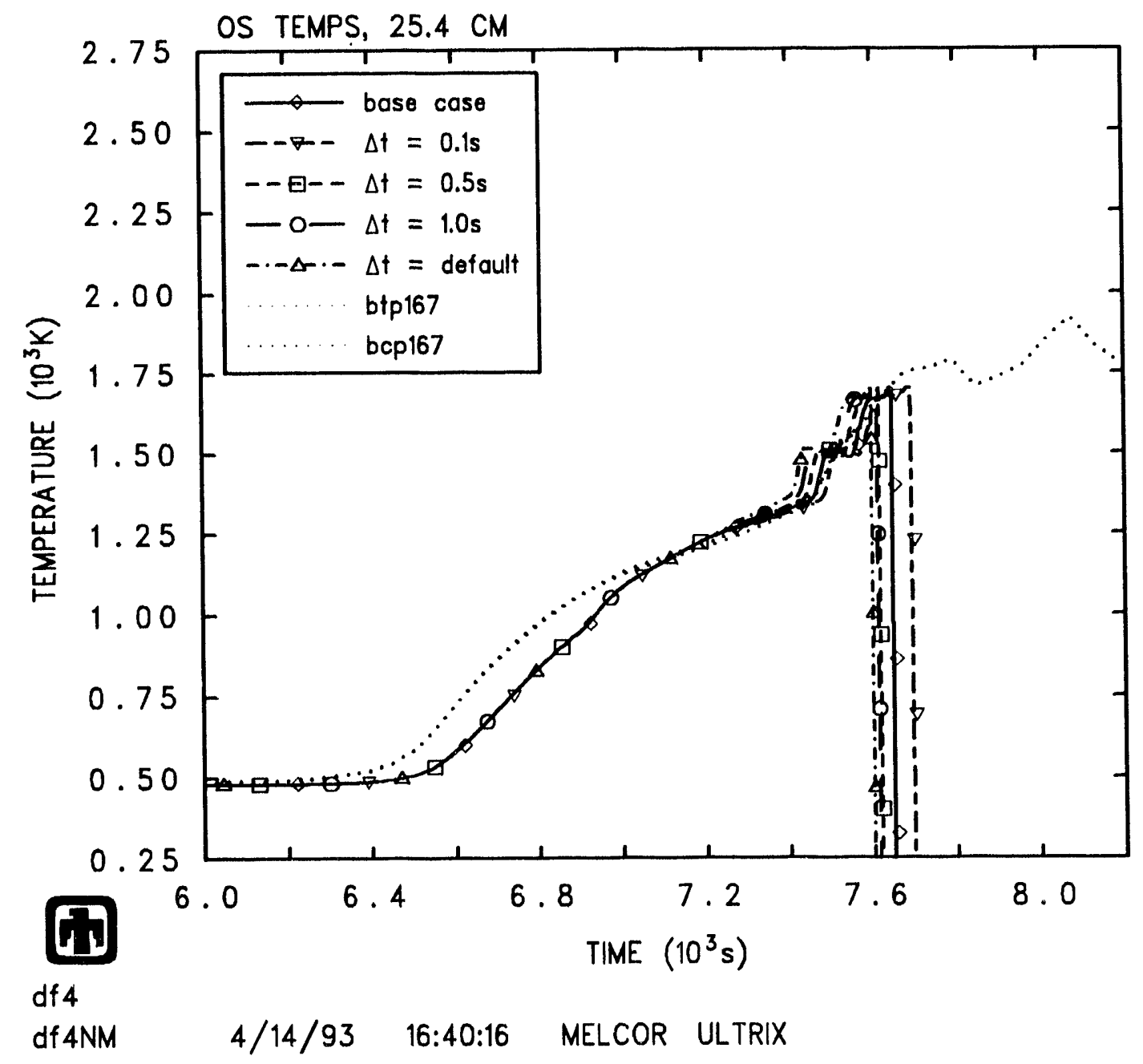

Figure 5.8.5. Control blade temperature response at the $25.4 \mathrm{~cm}$ plane for the time step sensitivity study, compared to base case and measured values. 


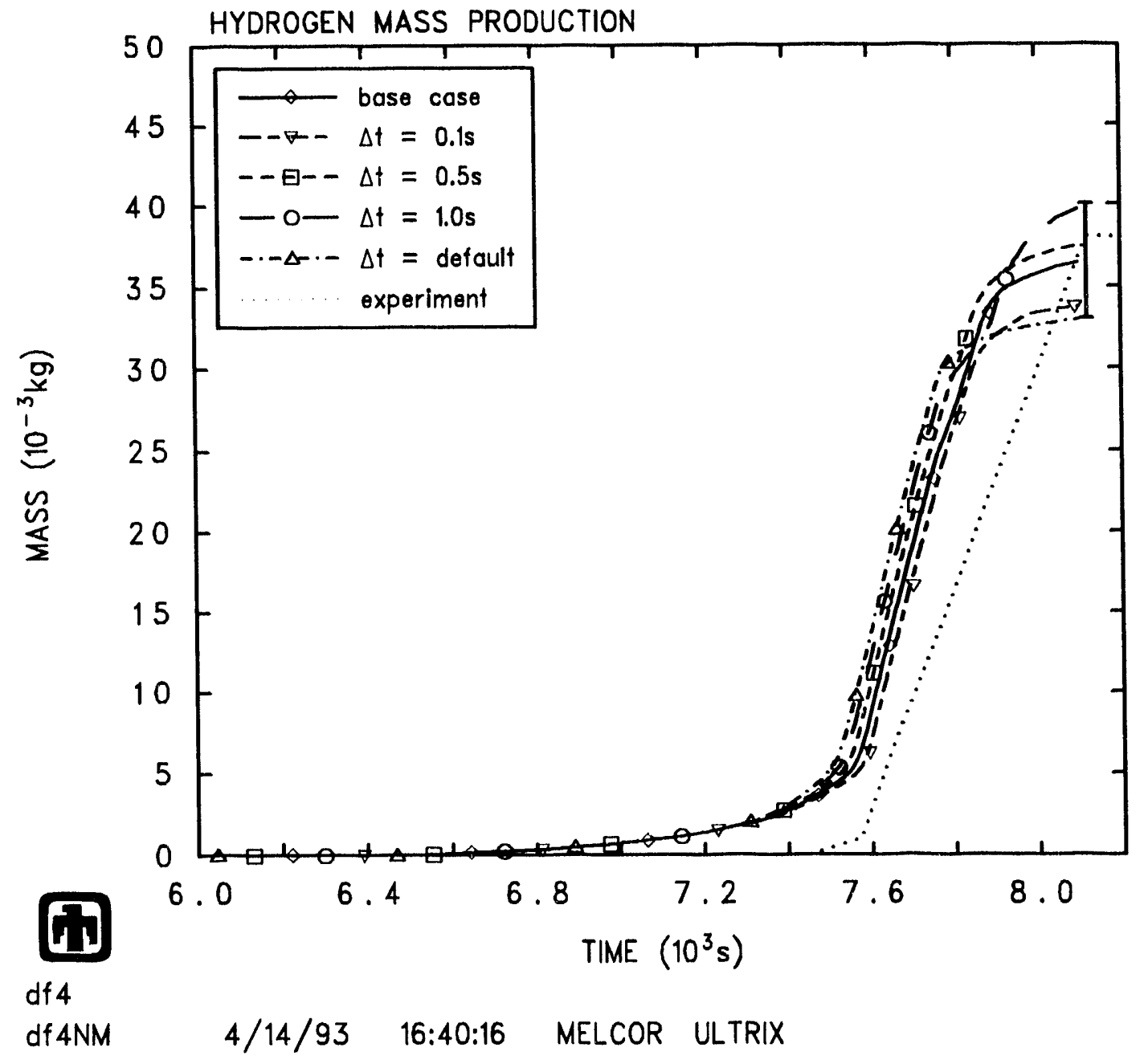

Figure 5.8.6. Hydrogen production for the time step sensitivity study, compared to base case and measured values. 
hydrogen production is shown in Figure 5.8.9. These figures show that there were no machine dependencies when running the DF-4 base case problem.

The final component states at 8100 seconds for all machines are shown in Figures 5.8.10 - 5.8.15. The final material locations were nearly identical across the machines, with the Cray and the SUN versions showing only slight variations and the bottom of the test bundle.

The overall system time steps and the COR package time steps taken by the various machines are shown in Figures 5.8.16 and 5.8.17, respectively. The system time steps were identical for all machines. The COR package time steps were identical and equal to the system time steps for most of the problem, but started to subcycle around 7550 seconds. At this time, a great deal of core component relocation was taking place (see Figures 4.2.4- 4.2.9), which would cause some COR package subcycling to occur. Since there were no significant differences in temperature and relocation results, the differences in COR package subcycling time step results were not significant.

The ending cycle number, the number of fallbacks, and the subcycle ratios for the CVH and COR packages are listed for each machine in Table 5.8.1. The only difference between the results are in the number of $\mathrm{COR}$ package subcycles per timestep on the Cray and the SUN compared with those on the other machines. Again, since the temperature and hydrogen production results agreed so well across the machines, these differences were insignificant.

The overall execution time for all the machines is shown in Figure 5.8.18. The execution time ratios, normalized to that of the fastest machine (the Hewlett Packard), are also listed in Table 5.8.1. Some features of the cpu time data to note are:

- The Hewlett Packard 755 was the fastest machine for running MELCOR on the DF-4 problem.

- The Hewlett Packard 755 was over twice the speed of the Cray X/MP-24.

- The slowest machine was the Gateway 486/DX2-50 PC, but not by much.

These execution time data also show the rapid evolution of workstation-class computers in terms of speed. The HP 755, a relatively new workstation, was somewhat faster than the IBM RS/6000 Model 550, which was in turn faster than the Cray $\mathrm{X} / \mathrm{MP}-24$. The $486 \mathrm{PC}$ had almost caught up to the slowest UNIX workstation on the list (in other assessment calculations, the $486 \mathrm{PC}$ is sometimes faster than the SUN Sparc2, and sometimes slower [11],[19]).

The MELCOR code runs on 386 PC's and VAX computer systems, but these systems were not treated in this assessment. In past assessments, the $386 \mathrm{PC}$ and VAX $8650 / 8700$ computers have been about half the speed of a 486 PC.

The execution times of the C.VH, COR and HS packages are compared with the overall execution tirre for the base case model in Figure 5.8.19. These data are for the run made on the DECStation 5000/240; data for other machines were quite similar. This figure reflects the emphasis placed on COR package calculations and the relatively simple $\mathrm{CVH}$ model in the base case input model. 


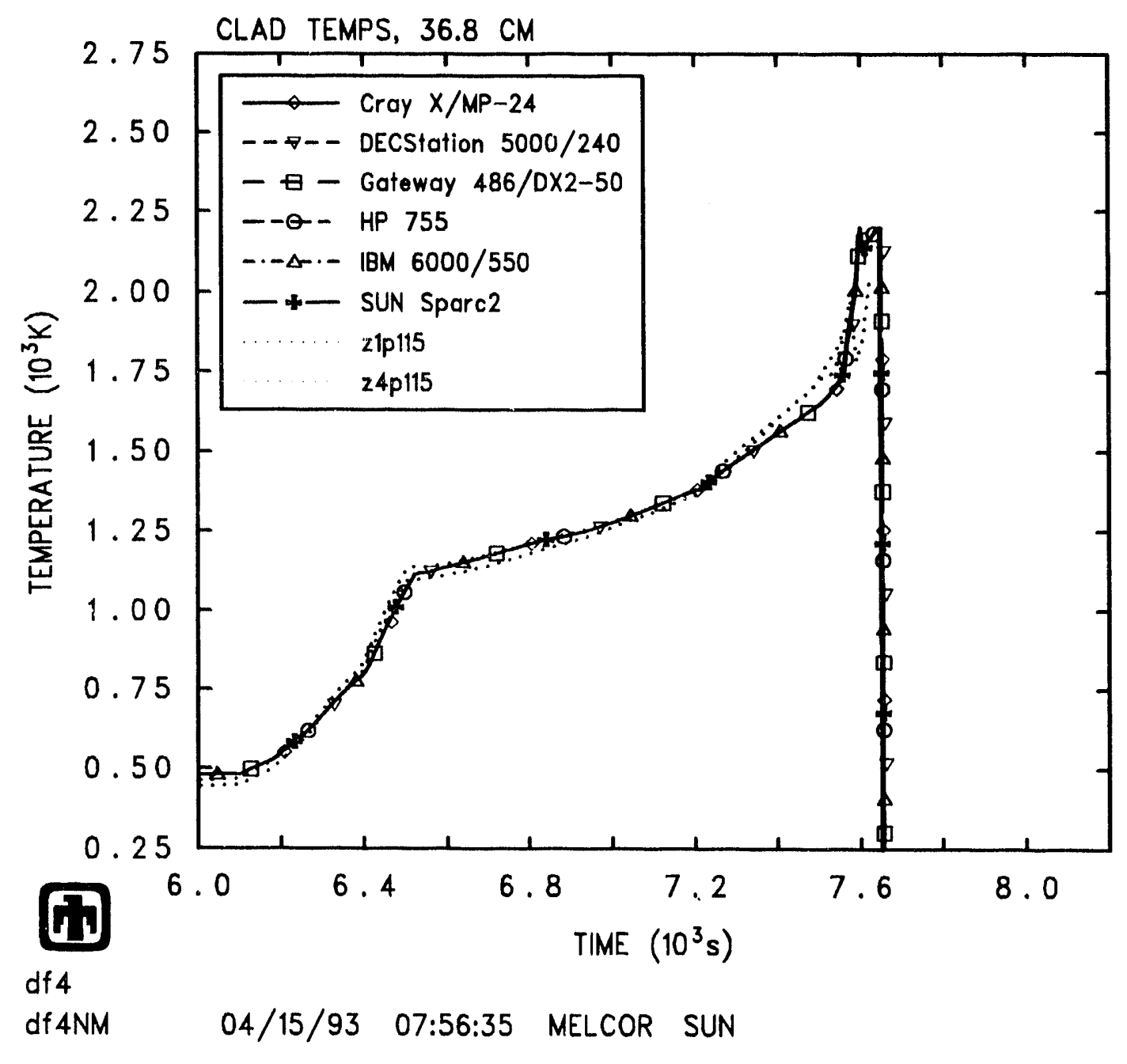

Figure 5.8.7. Cladding temperatures at the $36.8 \mathrm{~cm}$ plane for the six computers in the machine dependency sensitivity study, compared to measured values. 


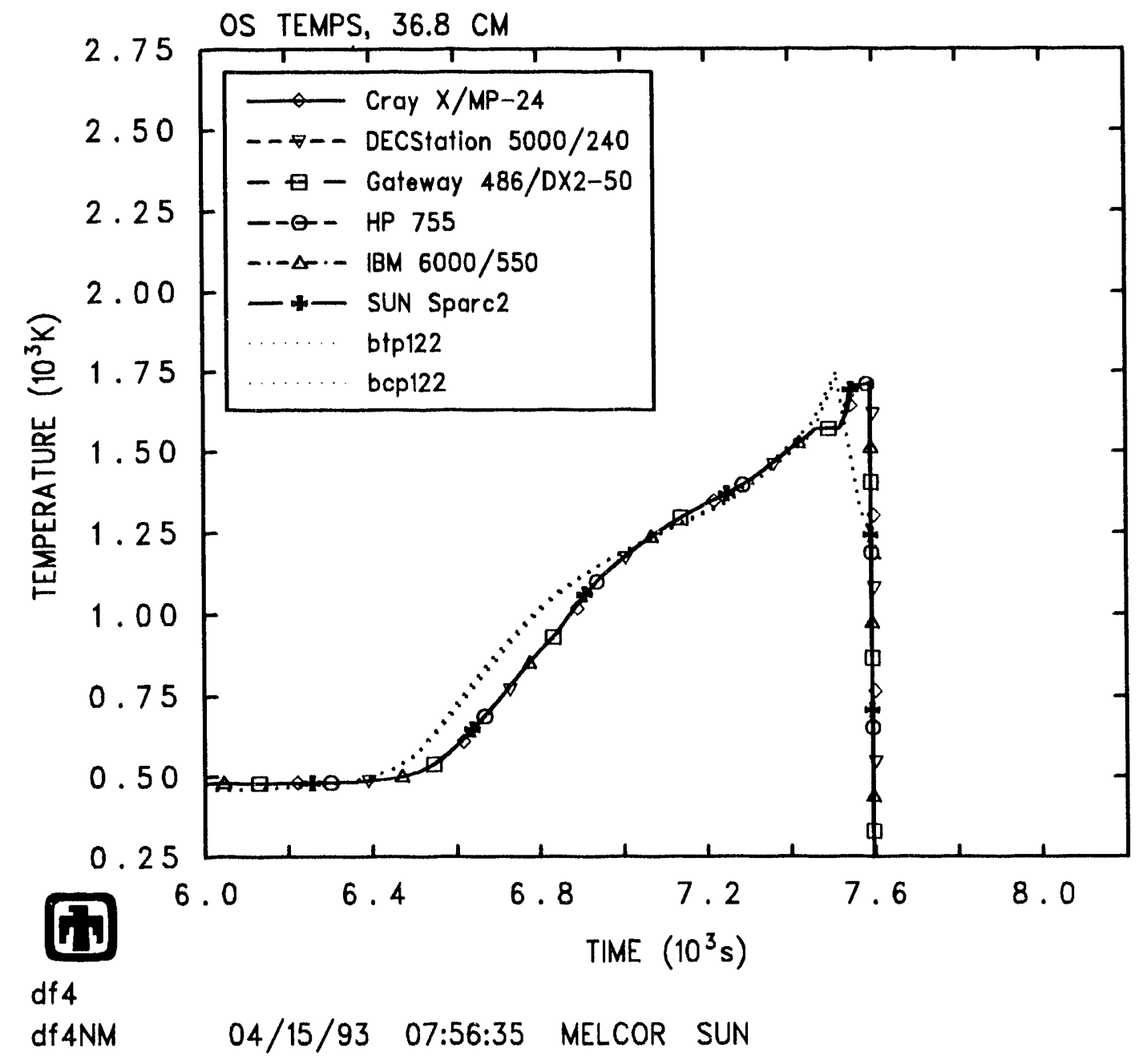

Figure 5.8.8. Control blade temperatures at the $36.8 \mathrm{~cm}$ plane for six computers in the machine dependency sensitivity study, compared to measured values. 


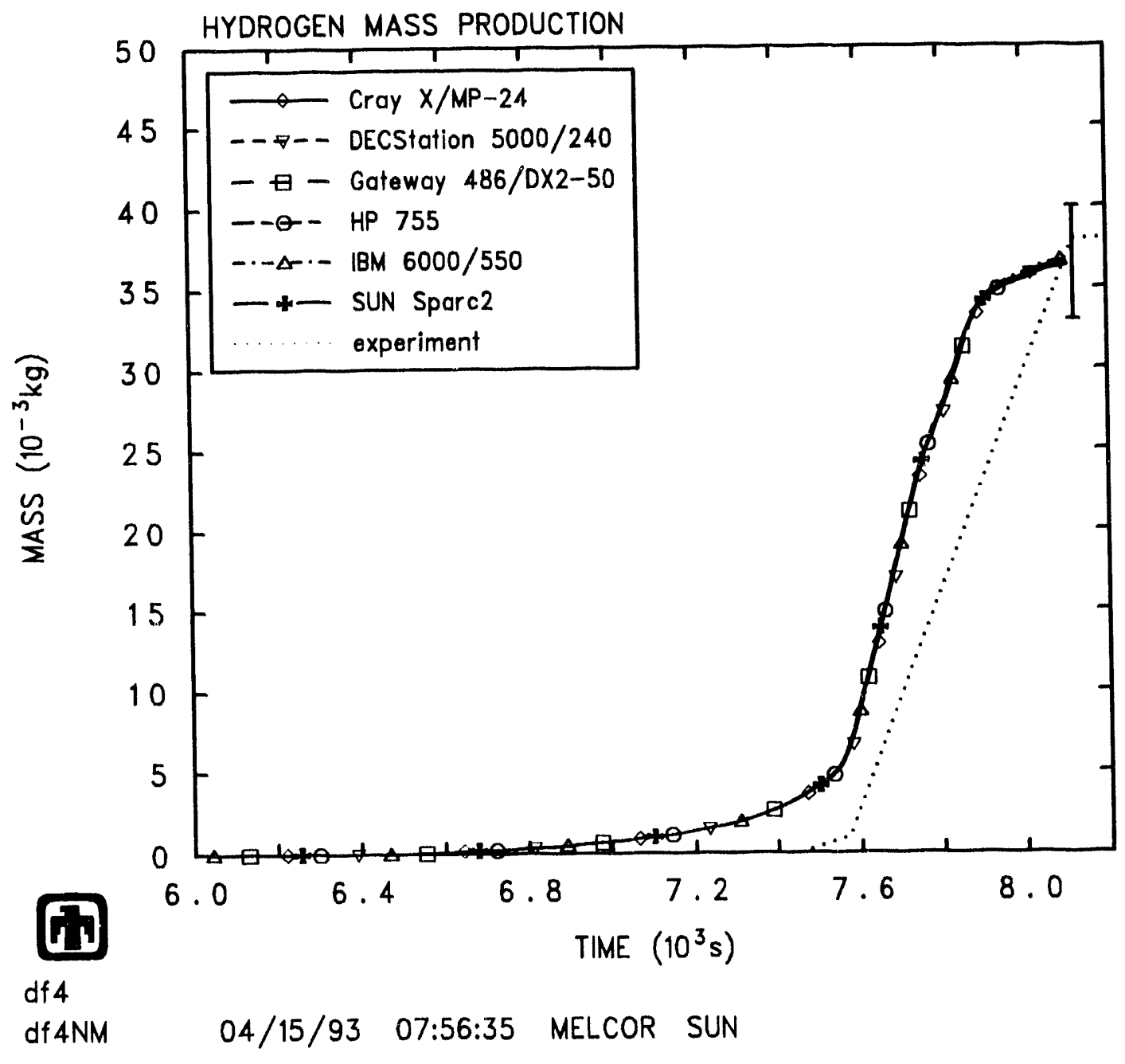

Figure 5.8.9. Hydrogen production for six computers in the machine dependency sensitivity study, compared to measured values. 

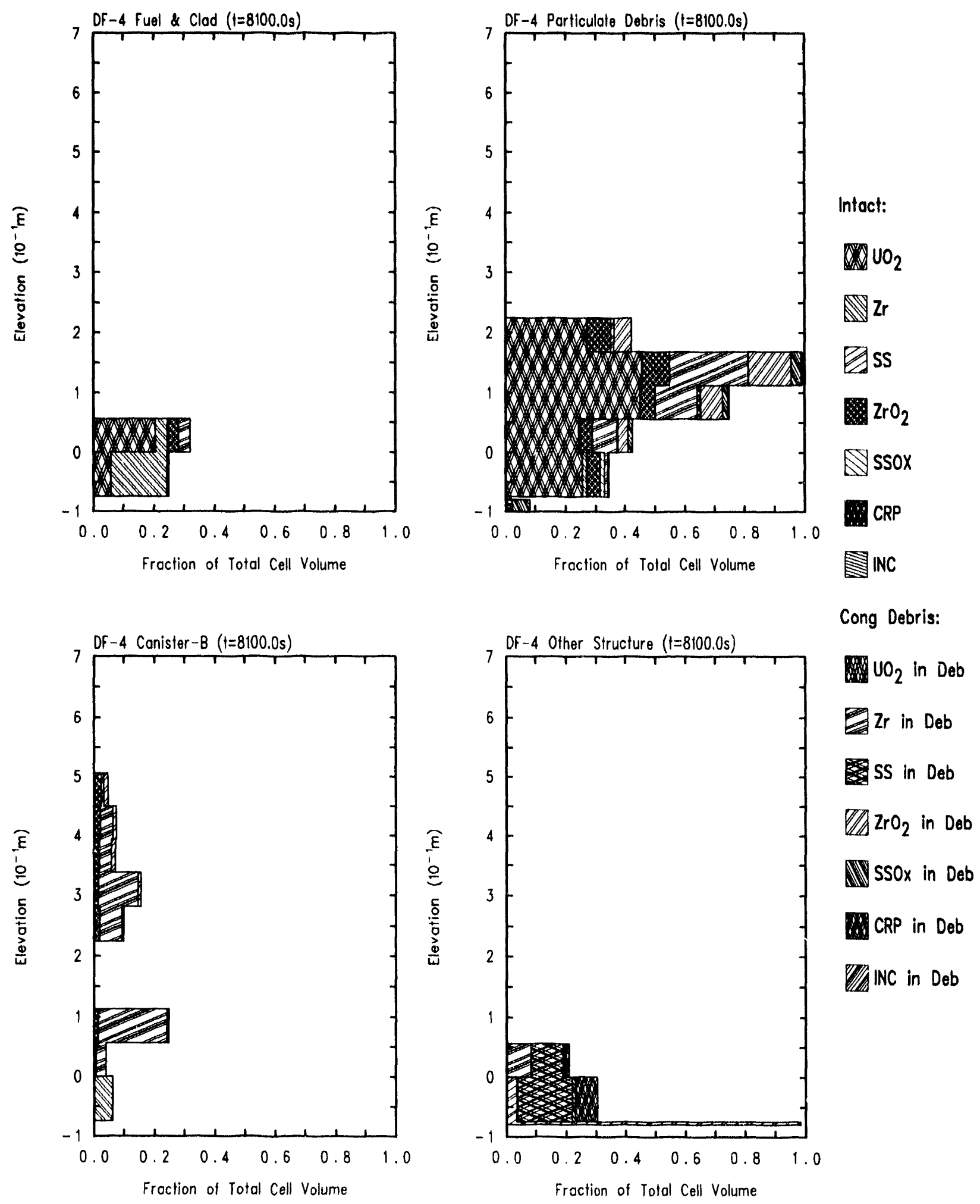

Figure 5.8.10. Component material locations at 8100 seconds for the Cray X/MP-24 case of the machine dependency sensitivity study. For an explanation of the format of this figure, see Section 4.2.1. 

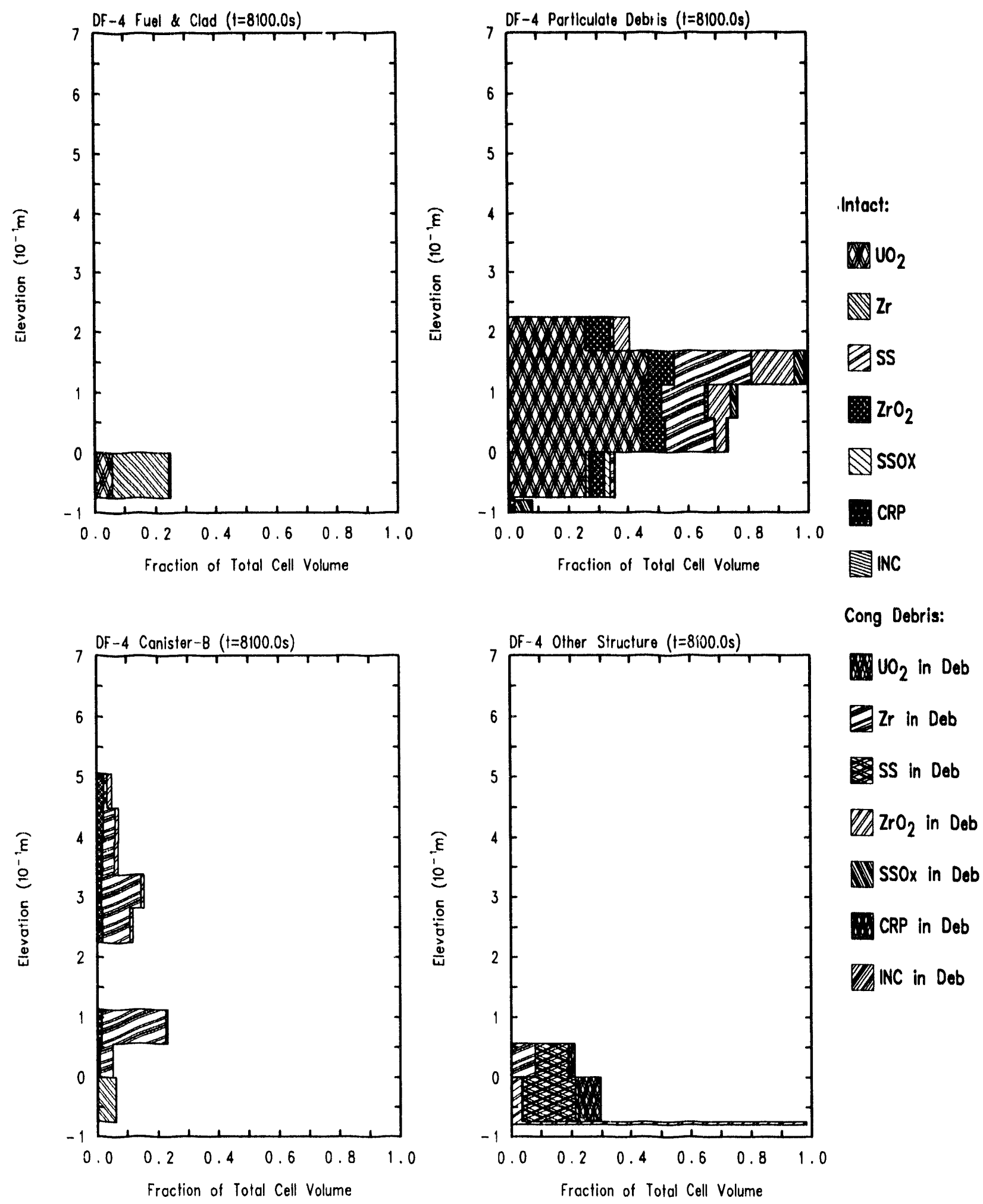

Figure 5.8.11. Component material locations at 8100 seconds for the DECstation $5000 / 240$ case of the machine dependency sensitivity study. For an explanation of the format of this figurs, see Section 4.2.1. 

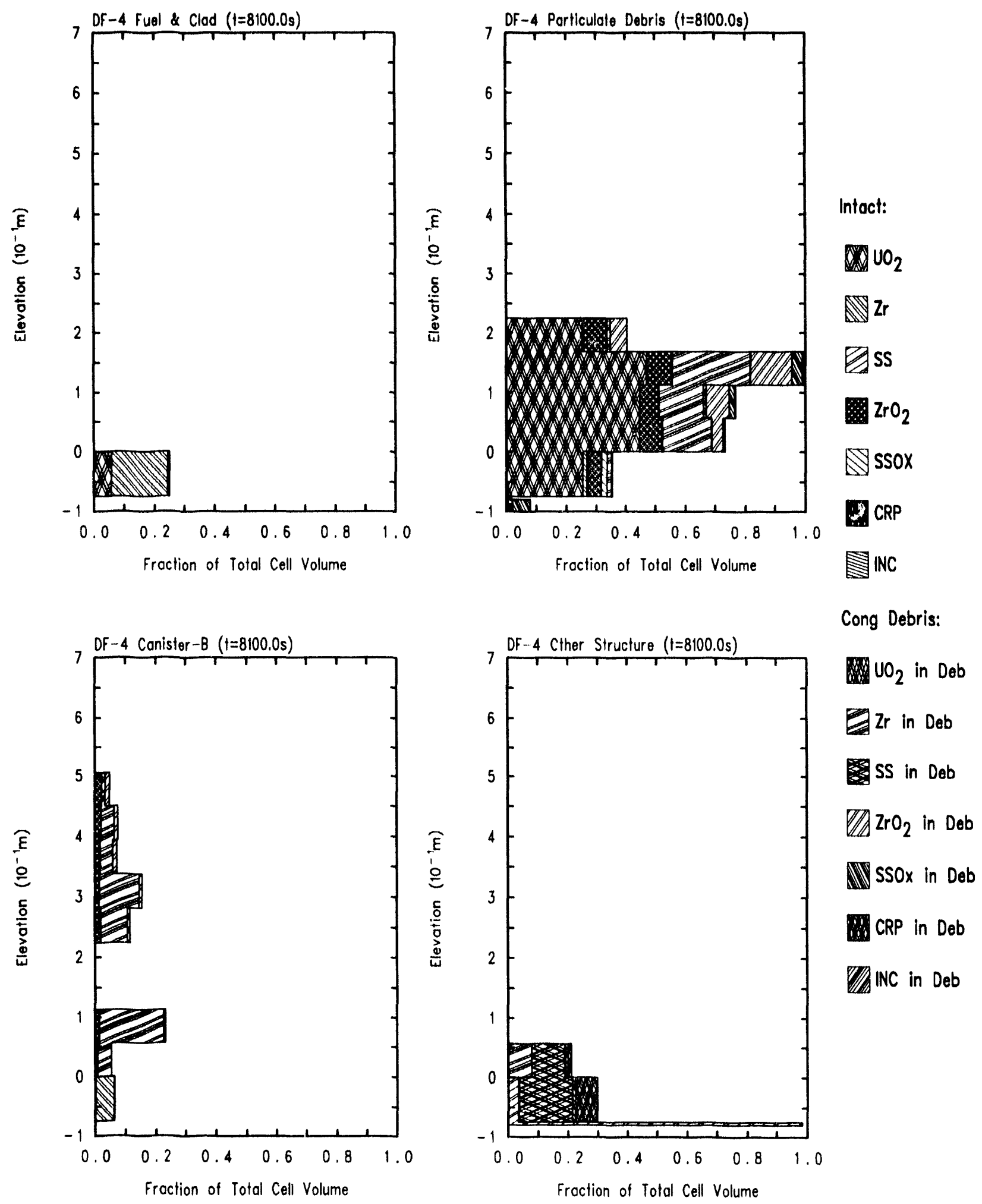

Figure 5.8.12. Component material locations at 8100 seconds for the Gateway 486/DX2-50 case of the machine dependency sensitivity study. For an explanation of the format of this figure, see Section 4.2.1. 

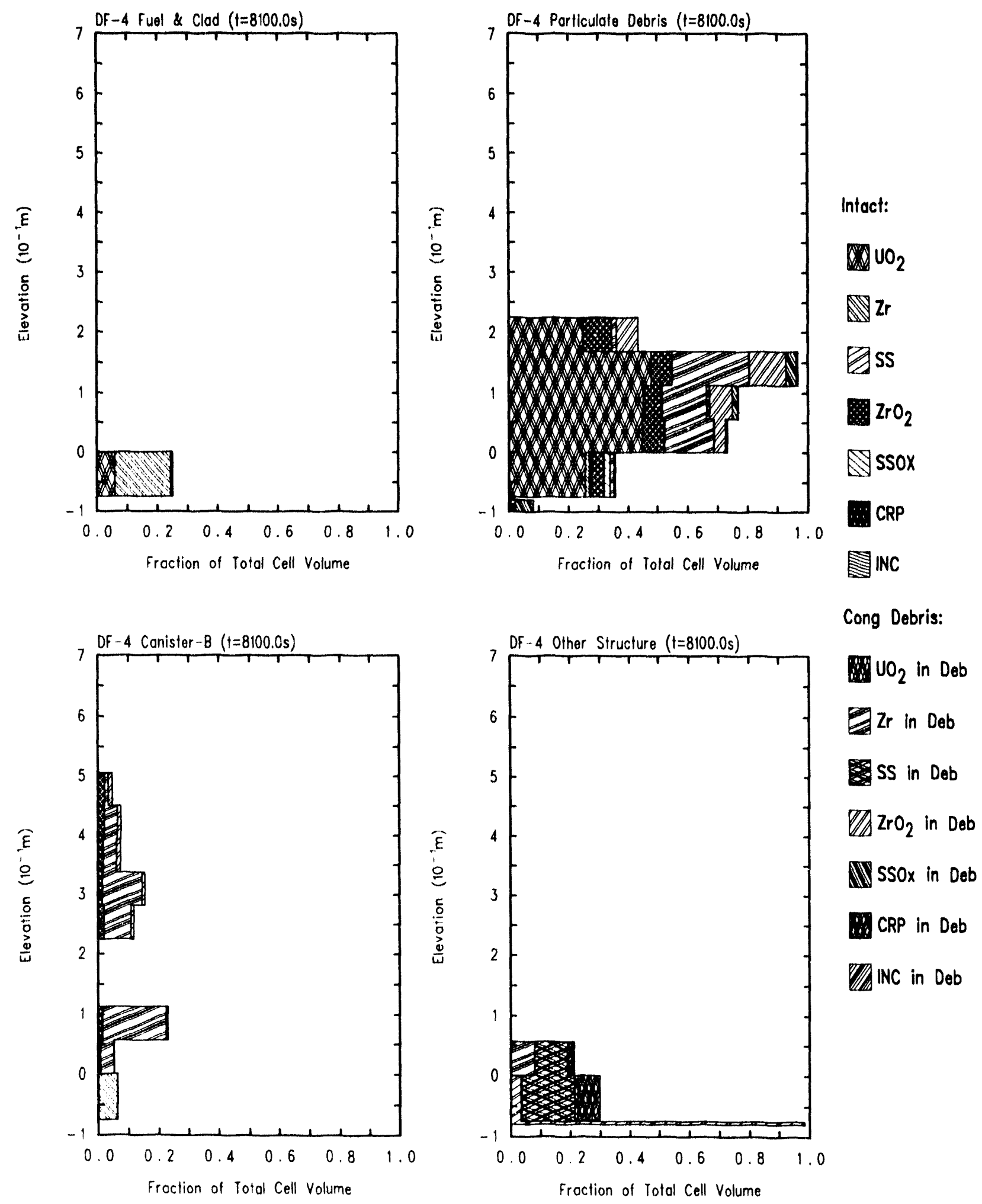

Figure 5.8.13. Component material locations at 8100 seconds for the Hewlett Packard 7.55 case of the machine dependency sensitivity study. For an explanation of the format of this figure, see Section 4.2.1. 

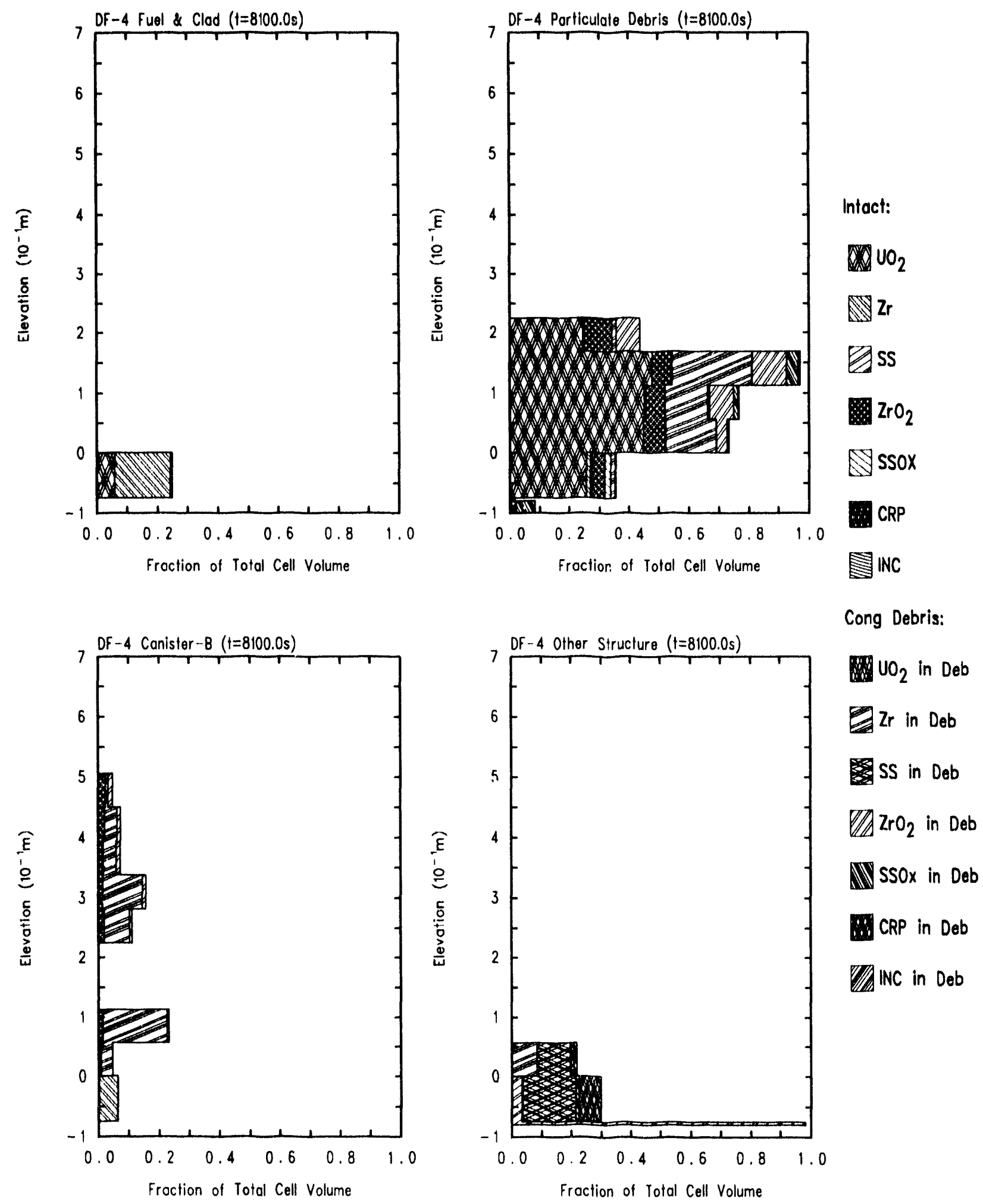

Figure 5.8.14. Component material locations at 8100 seconds for the IBM RS/6000 Model 550 case of the machine dependency sensitivity study. For an explanation of the format of this figure, see Section 4.2.1. 

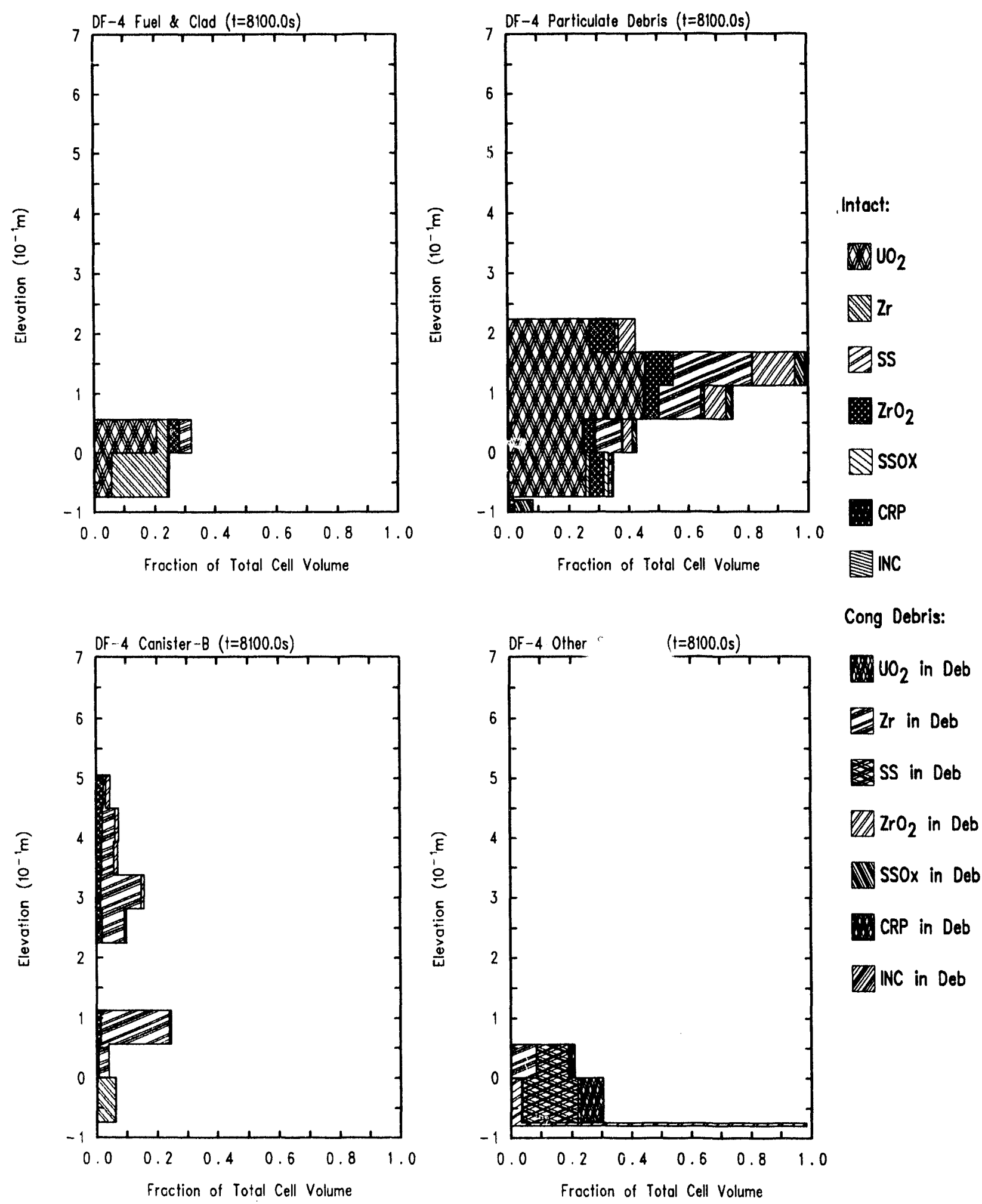

Figure 5.8.15. Component material locations at 8100 seconds for the SUN Sparcstation 2 case of the machine dependency sensitivity study. For an explanation of the format of this figure, see Section 4.2.1. 


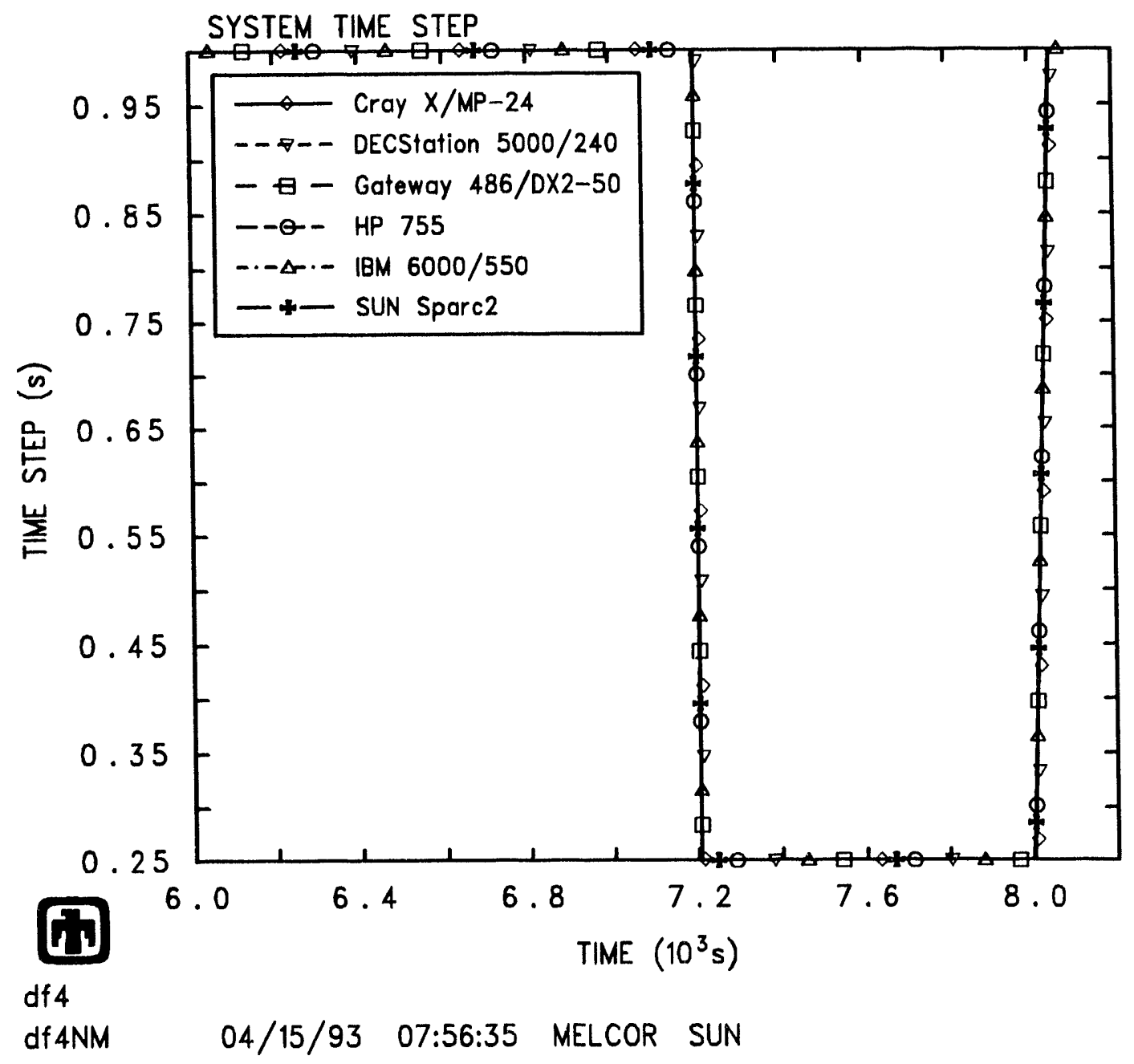

Figure 5.8.16. System time steps used by the six computers in the machine dependency sensitivity study. 


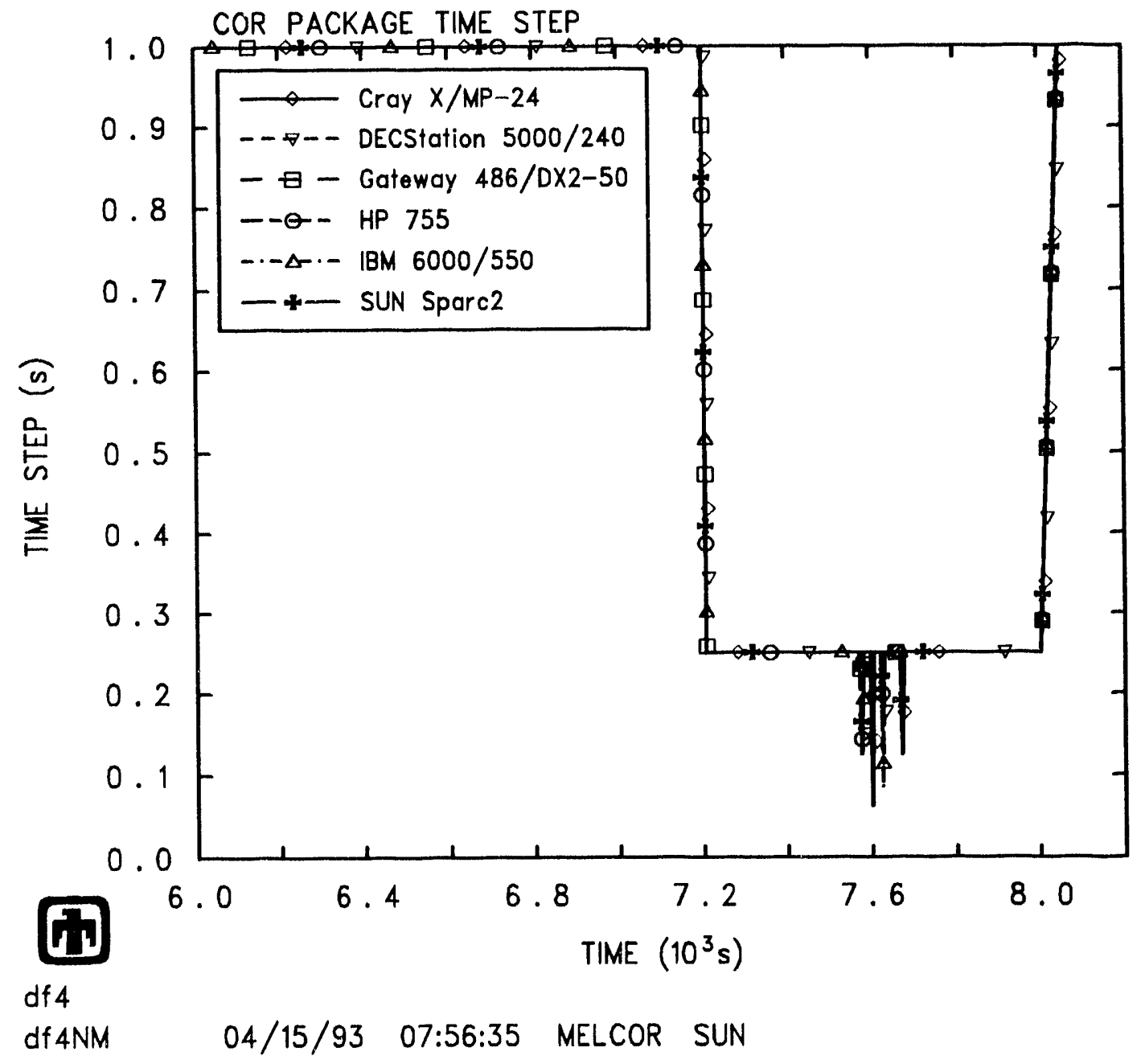

Figure 5.8.17. COR package time steps used by the six computers in the machine dependency sensitivity study. 


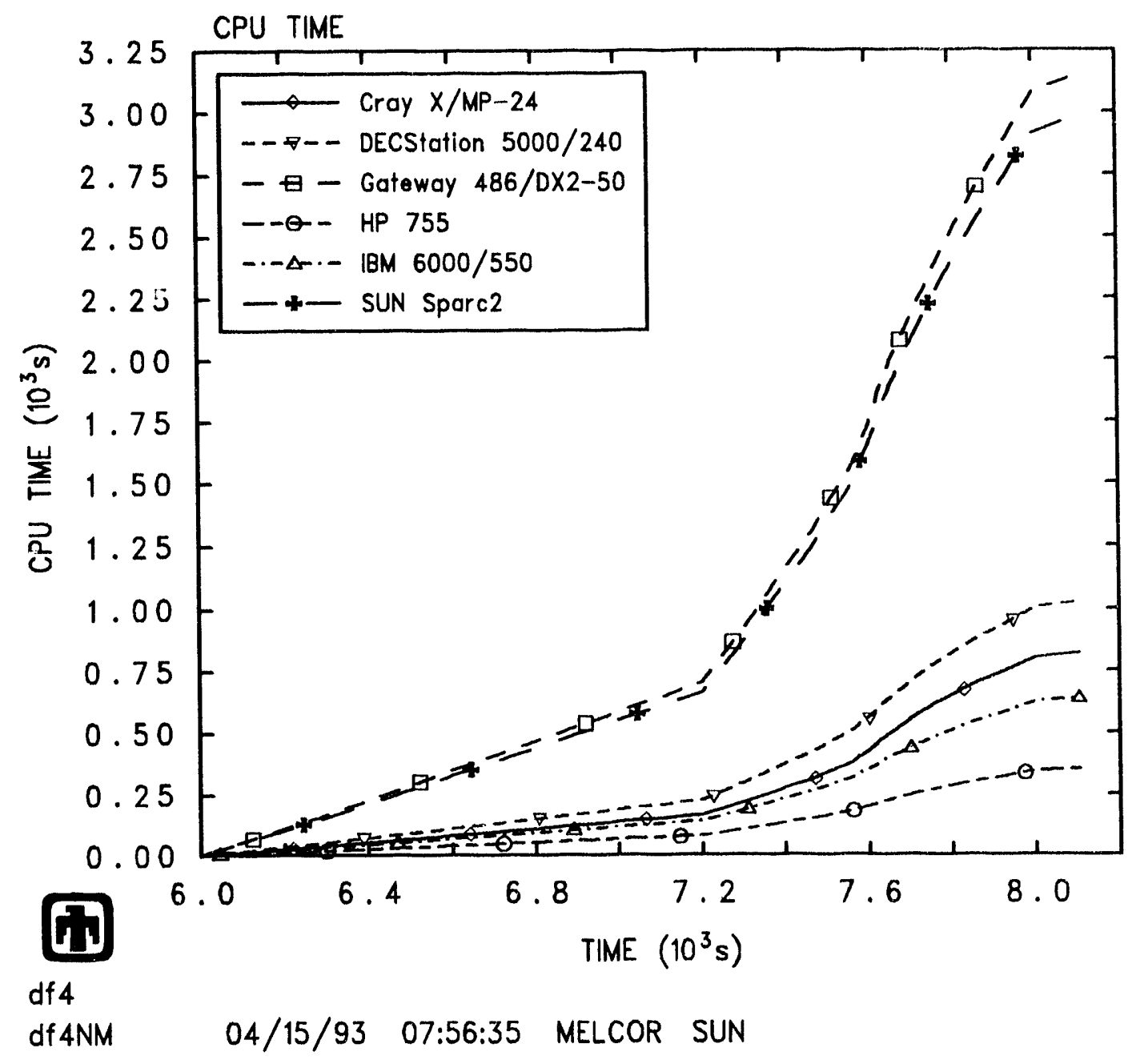

Figure 5.8.18. Overall execution time used by the six computers in the machine dependency sensitivity study. 


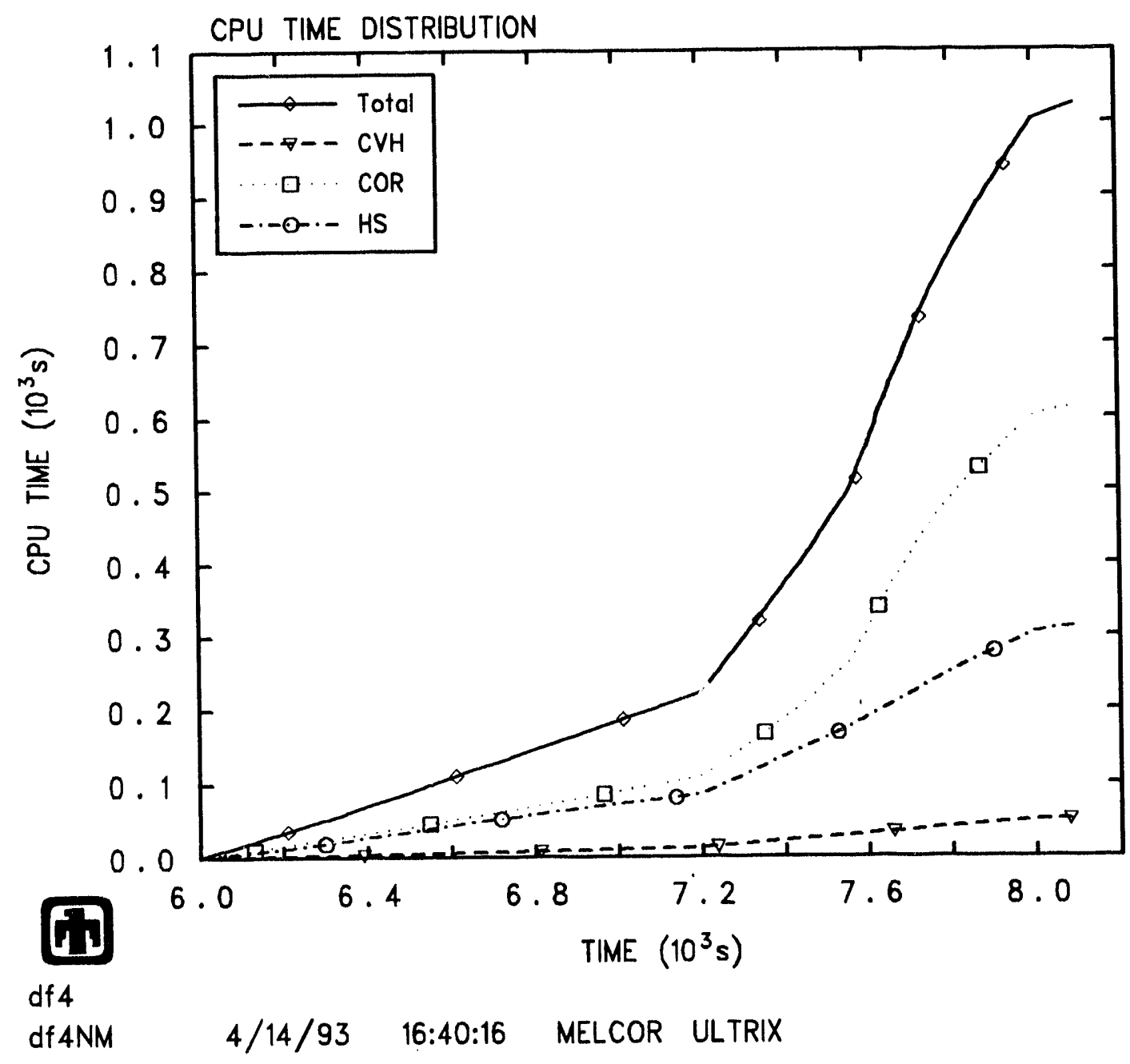

Figure 5.8.19. Package execution times for the DF-4 base case model executed on a DECStation 5000/240. 
Table 5.8.1. CVH and COR subcycle ratios used by the six computers in the machine dependency sensitivity study. Also shown are the total execution times and the total execution times normalized to that of the Hewlett Packard 755.

\begin{tabular}{c|c|c|c|c} 
Machine & $\begin{array}{c}\text { Avg. No. of } \\
\text { CVH Sub- } \\
\text { cycles per Cycle }\end{array}$ & $\begin{array}{c}\text { Avg. No. of } \\
\text { COR Sub- } \\
\text { cycles per Cycle }\end{array}$ & $\begin{array}{c}\text { Total } \\
\text { Execution } \\
\text { Time (s) }\end{array}$ & $\begin{array}{c}\text { Normalized } \\
\text { Execution } \\
\text { Time }\end{array}$ \\
\hline Cray X/MP-24 & 1.00 & 1.021 & 819.3 & 2.35 \\
DECStation 5000/240 & 1.00 & 1.018 & 1024.3 & 2.94 \\
Gateway 486/DX2-50 & 1.00 & 1.018 & 3144.3 & 9.04 \\
HP 755 & 1.00 & 1.018 & 348.0 & 1.00 \\
IBM RS/6000 Model 550 & 1.00 & 1.018 & 635.7 & 1.83 \\
SUN Sparc 2 & 1.00 & 1.021 & 2976.7 & 8.55
\end{tabular}




\section{Chapter 6}

\section{Comparison With Other Codes}

Several other codes have been used to model the DF-4 experiment. It is instructive to compare the results of the MELCOR DF-4 model to those of other codes, to evaluate the success of the parametric modeling approach taken by MELCOR. Results are discussed for the following codes: APRIL.MOD3 [20], BWRSAR/DF4 [21], MELPROG-PWR/MOD1 [15], and SCDAP/RELAP5/MOD2 [16].

The APRIL.MOD3 model [22] used twenty axial nodes and three radial rings for a total of sixty nodes to model the core region; 18 of 20 axial nodes were in the active fuel region. The BWRSAR/DF4 code model [23] used a total of 27 axial nodes in one radial ring, twenty of which were in the active fuel region. The MELPROGPWR/MOD1 DF-4 code model [8] used ten axial nodes and three radial rings for a total of thirty nodes to model the core region; seven of the ten axial nodes were in the active fuel region. The SCDAP/RELAP5 code model [24] used nine axial nodes, five in the active fuel region. These are comparable to the MELCOR model, which had a total of 14 axial levels, 9 of which are in the active fuel region.

The BWRSAR/DF4 and SCDAP/RELAP5 codes were each modified to improve the results of the DF-4 analyses [23],[24]. BWRSAI./DF4 required modifications to model the DF-4 geometry as well as the $\mathrm{B}_{4} \mathrm{C}$-stainless steel eutectic interaction. SCIAP/RELAP5 required modifications in order to model the radiation heat transfer between the channel box and the stainless steel control blade. The MELPROG and APRIL computer codes required no major modifications to model the DF-4 experiment, according to the published reports [8], [22]. The MELCOR code required some modifications as described in Chapter 7, but these changes were relatively minor and were included in the release version of MELCOR. However, this work was performed shortly after the addition of a new materials interactions (eutectics) model into the core package of MELCOR; this model greatly enhanced the ability of MELCOR to model the DF-4 experiment. Note also that the MELCOR model input was the result of some optimization using some of the sensitivity studies described in Chapter 5 . 


\subsection{Temperature Response}

The control blade temperatures calculated by MELCOR at the $36.8 \mathrm{~cm}$ and $25.4 \mathrm{~cm}$ planes are compared to experimental values and temperatures calculated by APRIL.MOD3, BWRSAR/DF4, MELPROG and SCDAP/RELAP5 in Figures 6.1.1 and 6.1.2, respectively. APRIL results were not available for the $25.4 \mathrm{~cm}$ plane. At $36.8 \mathrm{~cm}$, MELCOR temperatures were within the envelope of other codes everywhere except during the initial bundle heatup. Note that only MELCOR and BWRSAR/DF4 predicted some sort of melting behavior below $1700 \mathrm{~K}$, the melting point of stainless steel (melting behavior is indicated by a constant temperature over some period of time). BWRSAR/DF4 held the temperature at $1520 \mathrm{~K}$, which was the MELCOR default temperature for the stainless stecl- $\mathrm{B}_{4} \mathrm{C}$ cutectic reaction. MELCOR held the control blade temperature at $1570 \mathrm{~K}$ because this reaction temperature was increased by user input for the base case model.

MELCOR was farther from the measured temperatures during the early parts of the experiment at the $25.4 \mathrm{~cm}$ plane as well. However, MELCOR predicted the arrival of candling material and the subsequent heatup and failure better than MELPROG, BWRSAR/DF4 and SCDAP/RELAP5 at this level. BWRSAR/DF4 again modeled a small amount of control blade melting at this plane before $1700 \mathrm{~K}$ was reached. Note that MELCOR predicted earlier melt arrival and heatup, which was the result of its eutectic modeling.

The cladding temperatures calculated by MELCOR at the $36.8 \mathrm{~cm}$ and 25.4 $\mathrm{cm}$ planes are compared to experimental values and temperatures calculated by APRIL.MOD3, BWRSAR/DF4, MELPROG and SCDAP/RELAP5 in Figures 6.1.3 and 6.1.4, respectively. APRIL results were not ، vailable for the $25.4 \mathrm{~cm}$ plane. At the $36.8 \mathrm{~cm}$ plane, MELCOR-calculated temperatures were well within the envelope of the other codes; MELCOR also predicted the onset of rapid oxidation more accurately than all codes except APRIL.

At the $25.4 \mathrm{~cm}$ plane, MELCOR was within the envelope until midway through rapid oxidation, when the cladding heated up slightly faster than the other codes. Note that at both levels, MELCOR predicted cladding failure (indicated by the temperature dropping to zero) while the other codes show the cladding heating up to the zircaloy melting temperature of $2200 \mathrm{~K}$ and beyond. This could be because the other codes did not transfer cladding material to another component once it had melted, as did MELCOR.

The cladding temperature response at the $49.5 \mathrm{~cm}$ plane is shown in Figure 6.1.5. This figure shows that, indeed, the temperatures predicted at this plane by all codes were higher than the measured temperatures. This indicates that the power coupling relationship may have been in error at the upper-most plane. 


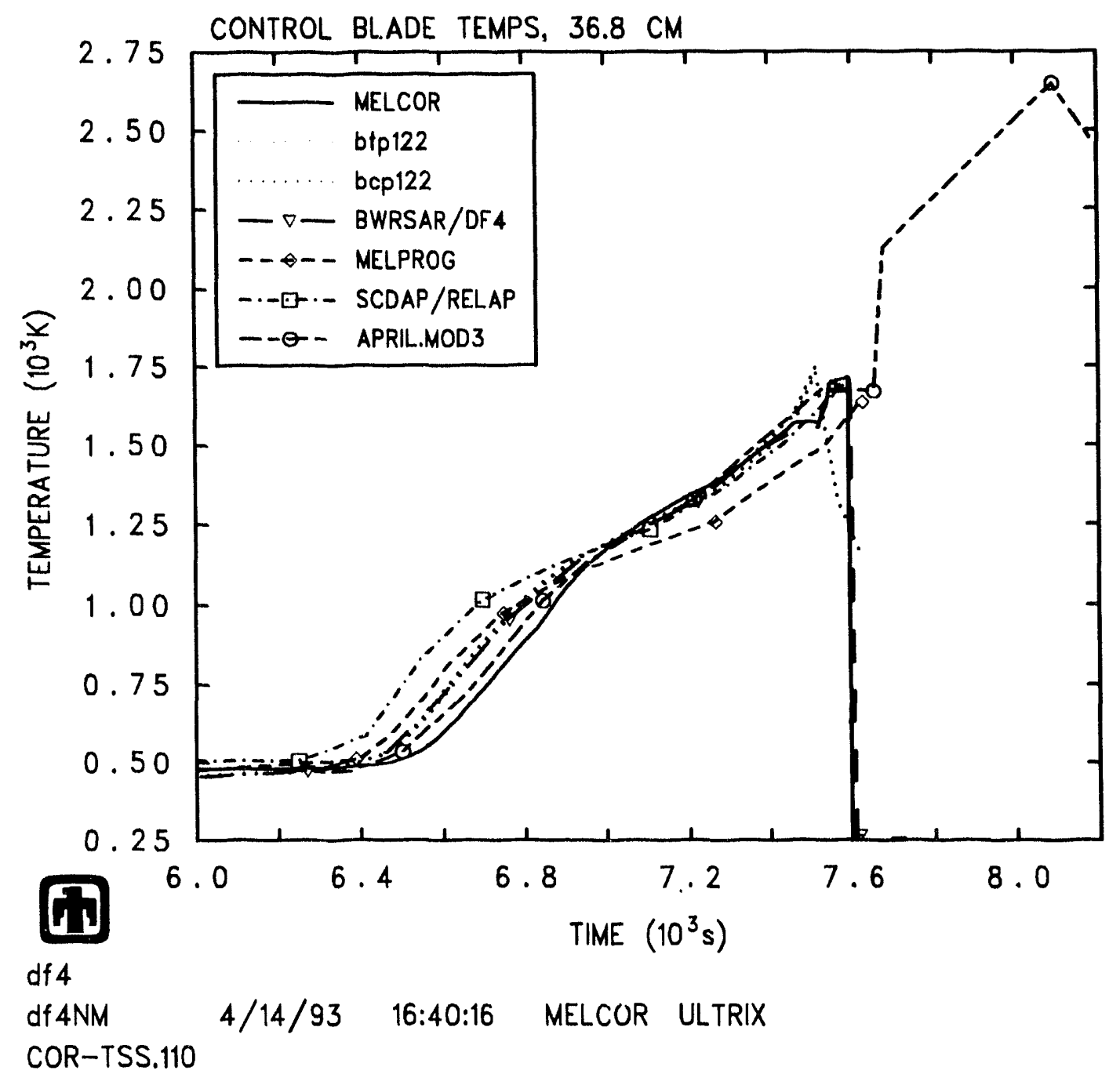

Figure 6.1.1. Comparison of control blade temperatures at the $36.8 \mathrm{~cm}$ plane calculated by APRIL, BWRSAR/DF4, MELPROG, SCDAP/RELAP, and MELCOR against experimental results. 


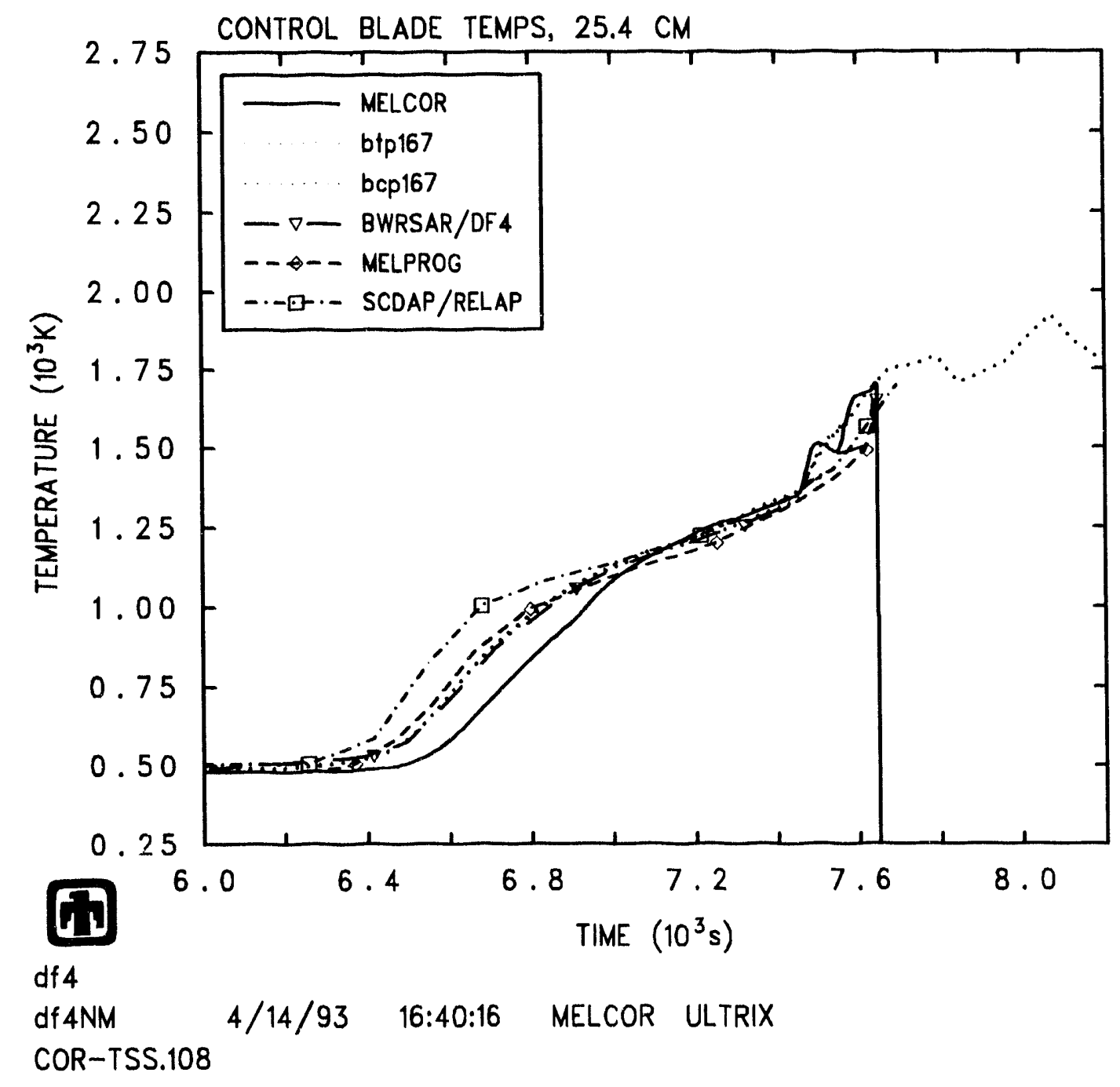

Figure 6.1.2. Comparison of control blade temperatures at the $25.4 \mathrm{~cm}$ plane calculated by APRIL, BWRSAR/DF4, MELPROG, SCDAP/RELAP, and MELCOR against experimental results. 


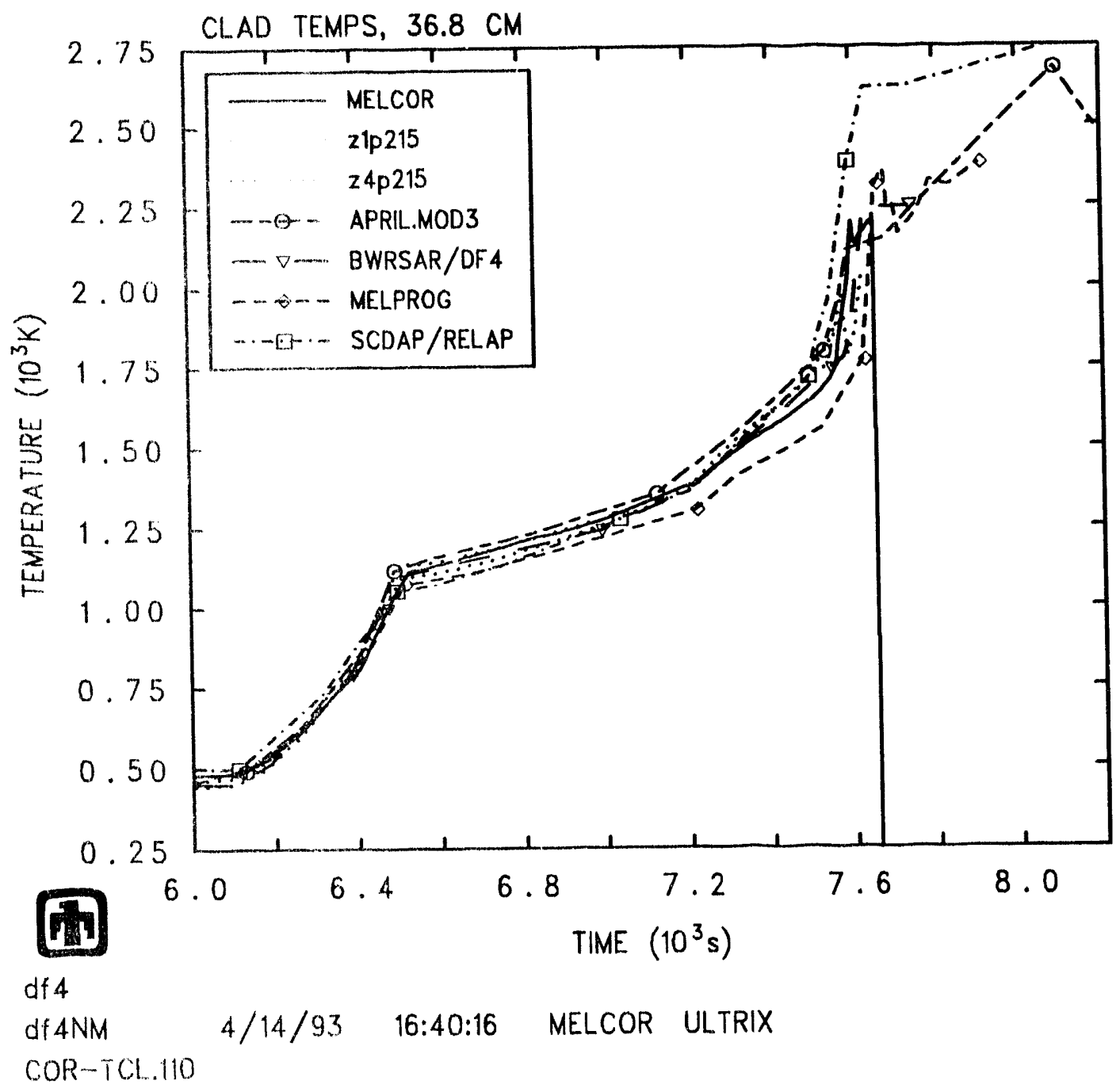

Figure 6.1.3. Comparison of cladding temperatures at the $36.8 \mathrm{~cm}$ plane calculated by APRIL, BWRSAR/DF4, MELPROG, SCDAP/RELAP, and MELCOR against experimental results. 


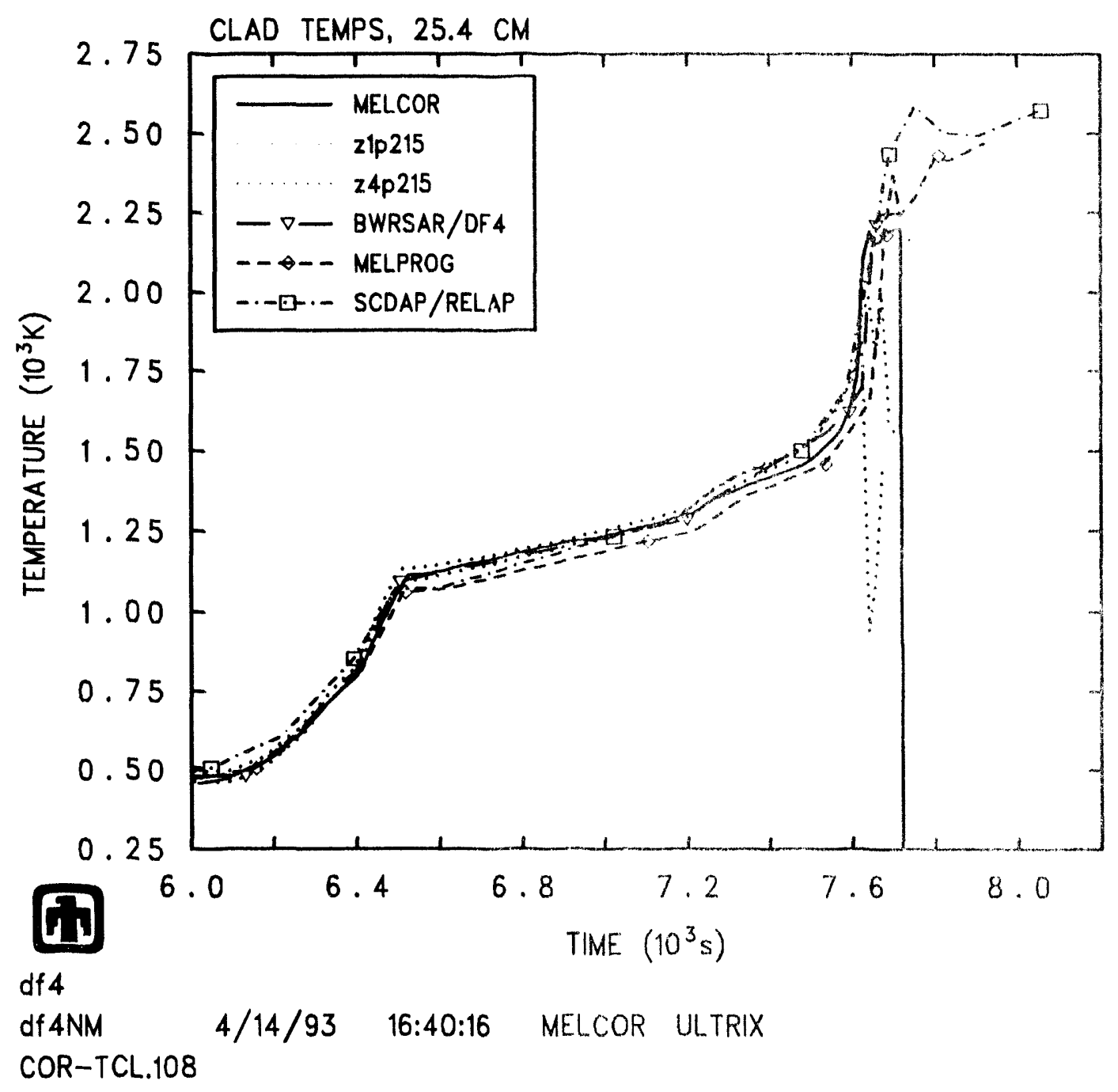

Figure 6.1.4. Comparison of cladding temperatures at the $25.4 \mathrm{~cm}$ plane: calculated by APRIL, BWRSAR/DFA, MELP'ROC,

SCDAP/RELAP, and MELCOR against experimental results. 


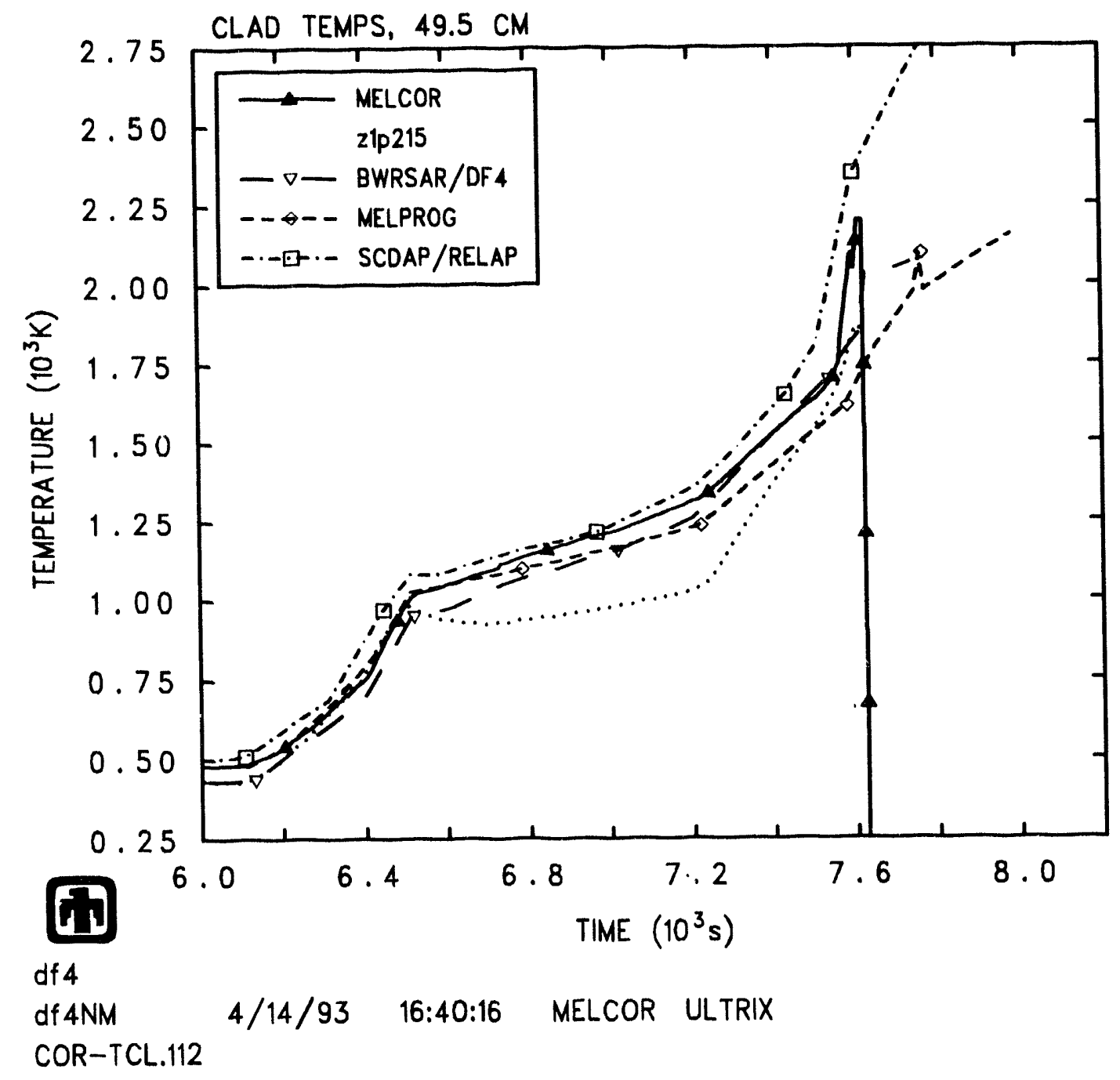

Figure 6.1.5. Comparison of cladding temperatures at the $49.5 \mathrm{~cm}$ plane calculated by APRIL, BWRSAR/DF4, MELPROG,

SCDAP/RELAP, and MELCOR against experimental results. 


\subsection{Hydrogen Production}

The hydrogen production rates calculated by APRIL.MOD3, BWRSAR/DF4, MELPROG, SCDAP/RELAP5, and MELCOR are compared to experimental measurements in Figure 6.2.1. Again MELCOR was within the calculational envelope during most of the experiment. All codes except SCDAP/RELAP5 predicted steamstarved conditions during the most vigorous oxidation. Note also that all codes predicted substantial hydrogen production before 7500 seconds, suggesting that the recombiner system used to measure hydrogen production was not sensitive enough to register a low hydrogen production rate. The increase in hydrogen production after 7800 seconds calculated by MELCOR was also calculated by the APRIL.MOD3 code, as shown in Figure 6.2.1. This effect was seen later on in the experiment, when the recombiner tubes were scmewhat depleted and were not measuring the true hydrogen production (see Figure 4.3.1). As explained in Section 4.3, this effect was probably real, and so the only codes predicting the correct amount of hydrogen production after 7800 seconds were APRIL and MELCOR.

The total integrated hydrogen production is shown in Table 6.2.1 for all codes except BWRSAR/DF4 (for which no data were available). Again, MELCOR was well within the envelope of other code results as well as the experimental uncertainty.

Table 6.2.1. Comparison of the total hydrogen generation calculated by MELCOR, APRIL, MELPROG and SCDAP/RELAP5 to experimental data. Experimental data is from the PIE [5], which gave a range of possible values.

\begin{tabular}{l|c}
\multicolumn{1}{c|}{ Code } & Total Hydrogen Produced \\
\hline Experiment & $38.0 \pm 4.0$ grams \\
MELCOR & 36.4 grams \\
APRIL & 37.5 grams \\
MELPROG & 51.0 grams \\
SCDAP/RELAP5 & 43.0 grams
\end{tabular}




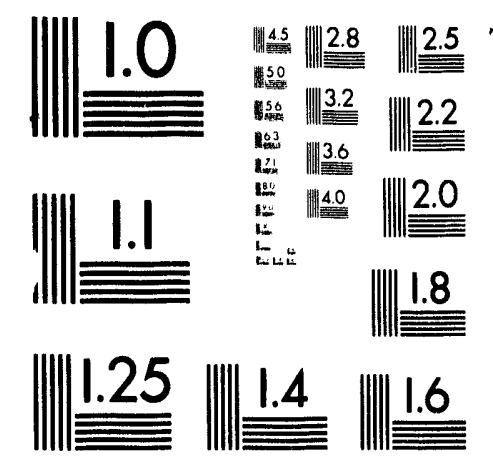




$$
\begin{aligned}
& \omega \\
& \stackrel{\rho}{\rho} \\
& \omega
\end{aligned}
$$




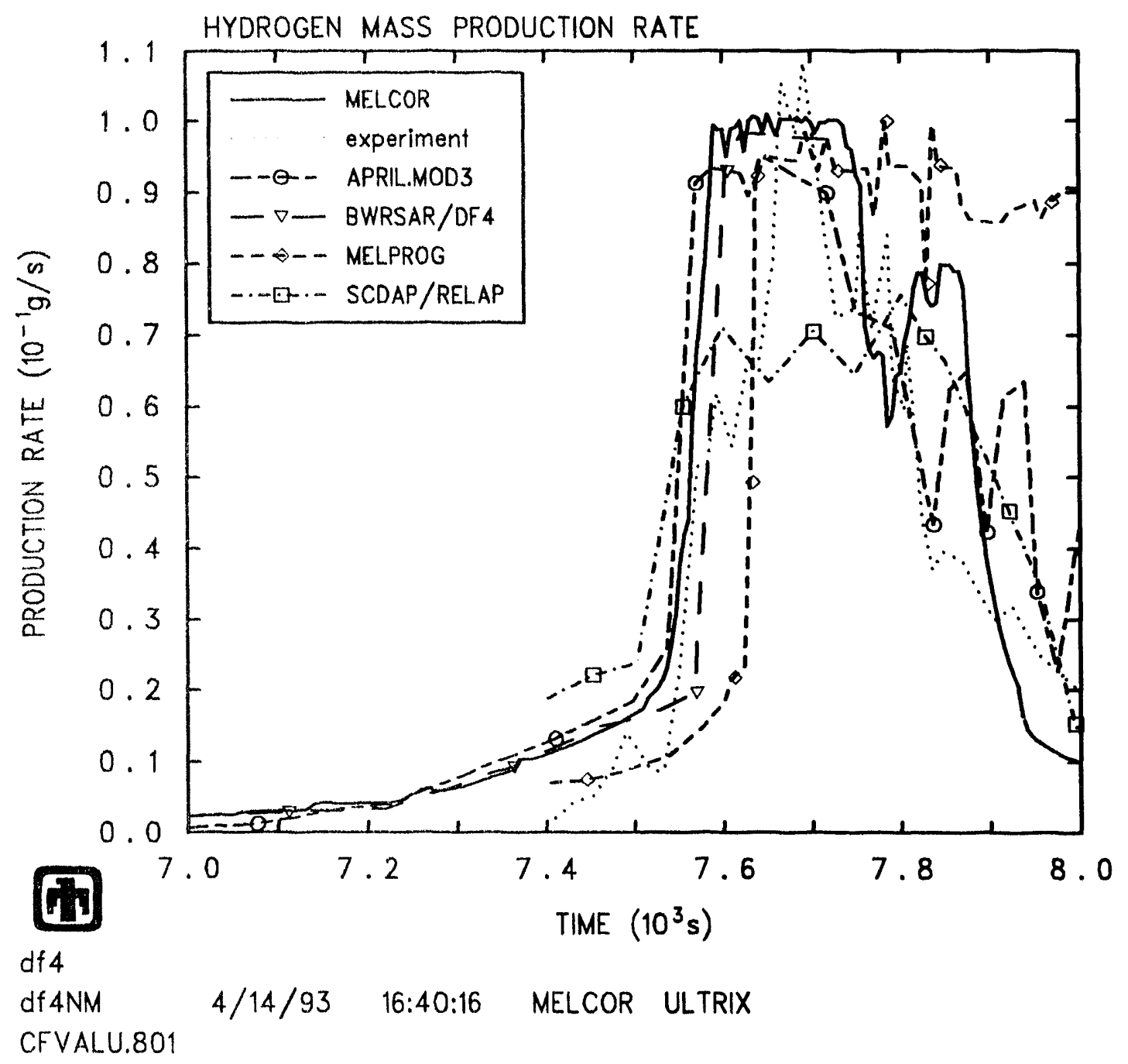

Figure 6.2.1. Comparison of the hydrogen generation rates calculated by APRIL, BWRSAR/DF4, MELPROG, SCDAP/RELAP, and MELCOR against experimental results. 


\subsection{Final State}

The final states of the test bundle predicted by MELPROG and MELCOR differed in the locations of fuel material, zircaloy and other core materials. 'The other codes gave no information on the predicted final state of the test bundle.

At 8100 seconds, MELPROG predicted some of the fuel material to be left standing, while MELCOR predicted the relocation of all fuel to the botton of the test bundle (see Figure 4.2.10). This was because MELCOR relocated fuel as soon as the intact cladding thickness fell below the minimum required to hold up fuel. MELPROC: also predicted the existence of a $\mathrm{ZrO}_{2}$ crust all the way up the bundle, while $\mathrm{MEL}$, COR relocated the oxidized cladding as well. The upper surface of dobis relocated to the bottom of the test bundle predicted by MELPRO(i was at approsinately $35 \mathrm{~cm}$, while MELCOR predicted the elevation of this surface to be approximately $22 \mathrm{~cm}$. Figures 4.2.11-4.2.14 suggest that the upper surface of the debris pile was somewhere in between these two levels, depending on the interpretation of what matcrials in the calculations correspond to the materials shown in the photographs. The existence of fuel material all the way up the test bundle, which was predicted by MLLPROG, was closer to the final experimental state than that predicted by MELCOR. 


\section{Chapter 7}

\section{Code Problems Identified}

Several code problems were identified during the course of this assessment project. These problems resulted in the submission of seven MELCOR Defect Investigation Reports (DIR's).

\subsection{Heat Transfer From Heat Structures to Channel Volume}

The COR package requires a radial boundary heat structure to be input for every axial level. This heat structure was assumed by the $\mathrm{dT} / \mathrm{dz}$ model to communicate with the bypass volume for heat transfer purposes. This represented the situation in a normal BWR core, where the radial boundary heat structures represent the core shroud, whose inside surface is cooled by the bypass region. In the DF-4 experiment, however, the channel region was adjacent to the radial boundary heat structures. The $\mathrm{dT} / \mathrm{dz}$ model was modified to use either the channel or bypass control volume to calculate heat transfer with the boundary heat structures, depending on which control volume was specified as the inside boundary volume for each heat structure. This change was implemented in version 1.8ME of MELCOR, as a result of DIR 1078.

\subsection{Divide By Zero in CVHBVT}

A time-specified volume was used to provide inlet steam to the MELCOR calculation. The conditions in this volume were specified to $\mathrm{CVH}$ on input records $\mathrm{CV} 100 \mathrm{Aj}$, and consisted of entries for PVOL, TATM, PH2O and MLFR.4. The entry for a noncondensible gas (MLFR.4) is required when TATM is used. However, in the case of DF-4, where there was no non-condensible gas flowing into the test bundle, the number input for this field had to be zero. In the routine CVHBVT, a normalization was done over all the non-condensible gasses, which divided by the total NCG mole fraction, which was zero in this case. A modification was made to set the toial NCG mole fraction to one if it was calculated as zero. This still resulted in normalized 
mole fractions equal to zero, without the floating point exception. This change was implemented in version $1.8 \mathrm{MN}$ as a result of DIR 1095.

\subsection{Internal File Length in MPDFVL}

In MPDFVL, a character variable CHERRS is used to build an error message, using an internal write statement. When the code attempted this operation, a fatal error was issued because the string being written into this variable was longer than the dimension of that variable. The dimension of this variable was increased to accommodate this error message. This change was implemented in version $1.8 \mathrm{MQ}$ as a result of DIR 1104 .

\subsection{Boundary Fluid Temperature Option in $\mathrm{dT} / \mathrm{dz}$}

The COR package $\mathrm{d} T / \mathrm{d} z$ model takes into account heat transfer with the radial boundary heat structures when calculating the temperature increase of steam flowing through the core control volumes. The temperature used to calculate heat transfer with the heat structures was the CVH bulk atmosphere temperature. In some cases, this bulk atmosphere temperature was very different from the local $\mathrm{d} T / \mathrm{d} z$ temperature calculated by the core package. This was particularly true when there wa:s a large temperature gradient across the core. This problem manifested itself in the heat transfer to the boundary heat structures in the lower two cells. There, the heat structures were in the neighborhood of $500 \mathrm{~K}$, while the bulk atmosphere temperature was over $1500 \mathrm{~K}$ (this temperature is normally close to the $\mathrm{dT} / \mathrm{d} z$ temperature in the uppermost core cell). As a result, the atmosphere was being cooled below the freezing point due to the artificially high heat transfer to the boundary heat structures.

A new option was added to the Heat Structures package to solve this problem. Each heat structure was allowed the option of using either the bulk atmosphere temperature or the local CGR $\mathrm{dT} / \mathrm{dz}$ temperature for calculating heat transfer to a core radial boundary heat siructure's atmosphere. 'This change solved the problem of freezing in the core, and also resulted in better agreement with experimental data, since it improved the calculation of heat transfer with the radial boundary heat structures (see Section 5.1.9). This change was implemented in version 1.8MY as a result of DIR's 1105 and 1120.

\subsection{Radiation Heat Transfer in $\mathrm{dT} / \mathrm{dz}$}

In the calculation of heat transfer with radial boundary heat structures in the $\mathrm{dT} / \mathrm{dz}$ model, only convective heat transfer was considered. In problems for which the radiation heat transfer dominated the overall heat transfer, as in some stages of DF-4, this resulted in a disagreement between the COR and HS packages on how much heat was transferred to the boundary heat structures. This resulted in the HS package removing too much energy from the core control volume as a result of 
COR calculating $\mathrm{dT} / \mathrm{dz}$ temperatures that were too high. A change accounting for radiation heat transfer with boundary heat structures in $\mathrm{dT} / \mathrm{dz}$ was requested in DIR 1139 and was implemented in MELCOR in version $1.8 \mathrm{NT}$.

\subsection{COR Energy Error Due to Fission Power}

The DF-4 experiment was driven by fission power from the ACRR. This fission power was represented by a tabular function in the MELCOR DF-4 input deck. The total fission power was specified along with peaking factors for each axial level, and these parameters were used to distribute fission power amongst the core cells. The fission power deposited in the fuel in each cell also depends on the amount of fuel inaterial $\left(\mathrm{UO}_{2}\right)$ in the cell. After major relocation started taking place in the DF-4 calculation, the actual amount of fission power deposited in the core was much less than the power specified in the tabular function. This resulted in a large COR package energy error, since the fission power specified in the tabular function was the value used for fission power accounting in the core package. This problem was reported in DIR 1140 and was implemented in MELCOR version 1.8NX. 


\section{Chapter 8}

\section{Conclusions}

MELCOR is a fully integrated, engineering-level computer code being developed at Sandia National Laboratories for the USNRC, that models the entire spectrum of severe accident phenomena in a unified framework for both BWRs and PWRs. As a part of an ongoing assessment program, MELCOR has been used to model the DF-4 Damaged Fuel experiment. DF-4 provided data for early phase melt progression in BWR fuel assemblies, particularly for phenomena associated with eutectic interactions in the BWR control blade and zircaloy oxidation in the canister and cladding.

The MELCOR base case input model for the DF-4 experiment consisted of 4 control volumes, 4 flow paths, and 15 heat structures. Fourteen core cells were modeled in 1 ring, with 9 cells in the active fuel region. The base case input model was based on the sensitivity studies performed as part of this assessment, and represented the best approximation of the experimental conditions and physical phenomena. Of the non-default MELCOR input parameters used in the base case model, the most important were the activation of the new eutectics model in MELCOR, those that changed the zircaloy melting temperature and the transition temperature for zircaloy oxidation rate, and the input enabling a new option for calculating heat transfer between core radial boundary heat structures and the core control volume atmosphere.

The base case model under-predicted control blade temperatures in the early parts of the experiment by almost $200 \mathrm{~K}$, but in later stages of the experiment, when all the core damage was taking place, calculated control blade temperatures corresponded almost exactly to measured values. Control blade failure times in most of the test bundle were predicted almost exactly compared to experimental data.

Cladding temperatures were predicted almost exactly compared to experimental data at all times and at all levels except for the upper-most axial level. MELCOR, and other codes, over-predicted temperatures in the upper-most axial level by close to the same amount $(250 \mathrm{~K})$ during the middle of the experiment, leading us to believe that the power coupling relationship did not predict power coupling well in this part of the core. Fuel failure times calculated by MELCOR corresponded almost exactly to experimental data.

Calculated canister temperatures were also very close to experimental data, after correcting this data for the time and temperature lags associated with the slowresponse thermocouples used for the canister. 
Material distribution plots for the melting and relocation portions of the experiment very clearly showed the effect of the $\mathrm{B}_{4} \mathrm{C}$-stainless steel eutectic interaction in the control blade. This reaction resulted in the first control blade failure around 7450 seconds, which was within 10 seconds of the first observed failure in the experiment. Eutectic dissolution of the canister wall was also evident and was responsible for the calculated failure of lower portions of the canister. Evidence of canister failure was seen in the post irradiation examination of the DF-4 test bundle.

The material distribution plots also showed clearly that in the DF-4 calculation, core materials relocated by axial level and not by component. That is, all components at a single axial level (fuel, clad, canister and control blade) melted and relocated before significant component relocation at other levels. This behavior could be strongly affected by code input parameters. For example, the default candling heat transfer coefficients resulted in the control material refreezing quite close to the axial location from which it melted. Behavior would be much different if the control blade materials were allowed to candle to the bottom of the test bundle, as they did during the DF-4 test. These results are important when considering the possibility of reactivity excursions due to control poison relocation without accompanying relocation of fuel material.

The amount of hydrogen production calculated by MELCOR was 36.4 grams, which was close to the amount derived from the PIE, which was $38.0 \pm 4.0$ grams. MELCOR calculated the autocatalytic oxidation reaction to begin sooner than was measured, and predicted 5 grams of hydrogen produced before the autocatalytic stage, compared to no hydrogen production measured in the experiment during that time. Other codes predicted early hydrogen production and early transition to the autocatalytic stage as well.

A large number of sensitivity studies were performed on MELCOR parameters, most of which were in the COR package, but also some in the HS and CVH packages. A study which deactivated the eutectics model showed clearly the benefits of using this model, as it predicted much different behavior of the $\mathrm{B}_{4} \mathrm{C}$ and did not show any canister dissolution. Hydrogen production without the eutectics model was well below the measured and MELCOR base case values. A sensitivity study which varied the eutectic temperature of the $\mathrm{B}_{4} \mathrm{C}$-stainless steel reaction by $\pm 50 \mathrm{~K}$ showed little variation of results. A study which used the default heat structure boundary fluid temperature option (which uses bulk atmosphere temperature instead of local $\mathrm{dT} / \mathrm{dz}$ temperatures for calculating heat transfer to the heat structures) resulted in much earlier component failure and poorer temperature agreement with experimental data. This study showed the usefulness of the new HS boundary fluid temperature option. Finally, a study on minimum oxide shell thickness and two other core relocation parameters in the COR package showed no variation in results until the critical minimum thicknesses for intact zircaloy and stainless steel were set to zero. After these parameters were changed, the final core material configuration showed the fuel pellet stacking observed in the PIE, but did not relocate any of the $\mathrm{ZrO}_{2}$ that resulted from cladding oxidation. Other sensitivity studies showed sensitivities to zircaloy properties, COR component view factors, allocation of canister mass to either the canister or canister-b component, candling heat transfer coefficient, COR 
and $\mathrm{CVH}$ nodalization, and slight sensitivity to $\mathrm{COR}$ and overall time steps. No sensitivities were found to minimum component mass, $\mathrm{B}_{4} \mathrm{C}$ oxidation modeling, and HS outer boundary temperature, and only slight variations in final material locations were found in the machine sensitivity study.

The results of the base case calculation were compared to those from four mechanistic codes: APRIL.MOD3, BWRSAR/DF4, MELPROG-PWR/MOD1, and SCDAP/RELAP5/MOD2. Early control blade temperatures predicted by MELCOR were lower than those predicted by other codes, but otherwise data calculated by MELCOR compared well with both the experimental data and those from the other codes.

This task resulted in improvements to the $\mathrm{COR} \mathrm{dT/dz}$ model, in particular with the addition of the HS boundary fluid temperature option. Several other code errors were uncovered and corrected during this analysis.

In conclusion, the MELCOR code achieved good agreement with experimental data. Temperatures, hydrogen production and control blade eutectic behavior compared well with data measured during the experiment and revealed from the PIE. The final configuration of debris calculated by MELCOR did not show the fuel pellet stacking observed in DF-4, but it was not clear that this configuration represented the behavior in full scale reactor cores during postulated severe accidents. This assessment task served to validate the part of the new MELCOR eutectics model that models $\mathrm{BWR}$ materials, in particular the $\mathrm{B}_{4} \mathrm{C}$-stainless steel reaction. 


\section{Bibliography}

[1] R. M. Summers, R. K. Cole Jr., E. A. Boucheron, M. K. Carmel, S. E. Dingman, and J. E. Kelly. MELCOR 1.8.0: A computer code for nuclear reactor severe accident source term and risk assessment analyses. Technical Report SAND900364, NUREG/CR-5531, Sandia National Laboratories, January 1991.

[2] R. D. Gasser, C. P. Fryer, R. O. Gauntt, A. C. Marshall, K. O. Reil, and K. T. Stalker. Damaged fuel experiment DF-1: Results and analysis. Technical Report SAND86-1030, NUREG/CR-4668, Sandia National Laboratories, January 1990.

[3] R. D. Gasser, C. P. Fryer, R. O. Gauntt, A. C. Marshall, K. O. Reil, and K. T. Stalker. Damaged fuel experiment DF-2: Results and analysis. Technical Report SAND86-1441, NUREG/CR-4669, Sandia National Laboratories, September 1992.

[4] C. P. Fryer, R. O. Gauntt, and A. W. Cronenberg. Damaged fuel experiment DF-3: Test results. Technical Report SAND87-1212, NUREG/CR-6006, Sandia National Laboratories, December 1992.

[5] Randall O. Gauntt, August W. Cronenberg, Carol P. Fryer, Susan C. Bourcier, Ronald D. Gasser, Kenneth O. Reil, and Jay J. Weingardt. Damaged fuel experiment DF-4: Test results. Sandia National Laboratories. to be published.

[6] R. O. Gauntt, R. D. Gasser, and L. J. Ott. The DF-4 BWR fuel damage experiment in ACRR with a BWR control blade and channel box. Technical Report SAND86-1443, NUREG/CR-4671, Sandia National Laboratories, November 1989.

[7] R. C. Smith. The core package eutectic model. Sandia National Laboratories. Letter report to R. B. Foulds, NRC, November 111992.

[8] Rodney C. Schmidt. MELPROG-PWR/MOD1 analysis of the DF-4 experiment. SAND90-1098, NUREG/CR-5578, Sandia National Laboratories. to be published.

[9] D. L. Hagrman, G. A. Reymann, and R. E. Mason. MATPRO version 11 (revision 1): A handbook of materials properties for use in the analysis of light water reactor fuel rod behavior. Technical Report TREE-1280 Rev. 1, NUREG/CR0497, EG\&G Idaho, February 1980. 
[10] L. N. Kmetyk. MELCOR 1.8.1 assessment: ACRR source term experiments ST1/ST-2. Technical Report SAND91-2833, Sandia National Laboratories, April 1992.

[11] L. N. Kmetyk. MELCOR 1.8.1 assessment: LOFT integral experiment LP-FP-2. Technical Report SAND92-1373, Sandia National Laboratories, December 1992.

[12] S. Hagen et al. Results of SFD experiment CORA-13 (OECD international standard problem 31). Technical Report KfK 5054, Kernforschungszentrum Karlsruhe, February 1993.

[13] Robert J. Gross, Samuel L. Thompson, and Gail M. Martinez. MELCOR 1.8.1 calculations of ISP31: The CORA13 experiment. Technical Report SAND922863, Sandia National Laboratories, June 1993.

[14] K. E. Washington et al. Reference manual for the CONTAIN 1.1 code for containment severe accident analysis. Technical Report SAND91-0835, NUREG/CR5715, Sandia National Laboratories, July 1991.

[15] S. S. Dosanjh and et al. MELPROG-PWR/MOD1: A two-dimensional mechanistic code for analysis of reactor core melt progression and vessel attack under severe accident conditions. Technical Report SAND88-1824, NUREG/CR-4909, Sandia National Laboratories, 1987.

[16] C. M. Allison and E. C. Johnson. SCDAP/RELAP5/MOD2 code manual: Volume 1: SCDAP code structure, systems models, and solutions methods. Technical Report EGG-2555, NUREG/CR-5273, EG\&G Idaho, Inc., July 1989.

[17] R. M. Summers and R. C. Smith. Core (COR) package reference manual, version 1.8.2. Sandia National Laboratories, January 1993.

[18] V. F. Urbanic and T. R. Heidrick. High-temperature oxidation of zircaloy-2 and zircaloy-4 in steam. J. Nuc. Matls., 75:251-261, 1978.

[19] L. N. Kmetyk. MELCOR 1.8.1 assessment: Marviken- $V$ aerosol transport tests ATT-2b/ATT-4. Technical Report SAND92-2243, Sandia National Laboratories, January 1993.

[20] R. T. Lahey Jr. and M. Z. Podowski. Degraded core modeling APRIL.MOD3 severe accident code. Technical Report ESEERCO PROJECT EP84-4, 1990. Final Report.

[21] S. A. Hodge and L. J. Ott. Boiling water reactor severe accident response (BWRSAR) code description and assessment. Technical Report NUREG/CR-5318, Oak Ridge National Laboratory. to be published.

[22] S. W. Kim, M. Z. Podowski, and R. T. Lahey, Jr. Numerical simulation of DF-4 and CORA-16/17 severe fuel damage experiments using APRIL.MOD3. In Proceedings of the 5th International Topical Meeting on Reactor Thermal/Hydralics (NURETH-5), pages 1497--1506, September 1992. 
[23] L. J. Ott. Post-test analyses of the DF-4 BWR experiment using the BWRSAR/DF4 code. Oak Ridge National Laboratory letter report, August 1989.

[24] Judith K. Hohorst and Chris M. Allison. DF-4 analysis using SCDAP/RELAP5. Nuclear Technology, 98:149-159, May 1992. 


\section{Appendix A}

\section{Base Case Input Deck}

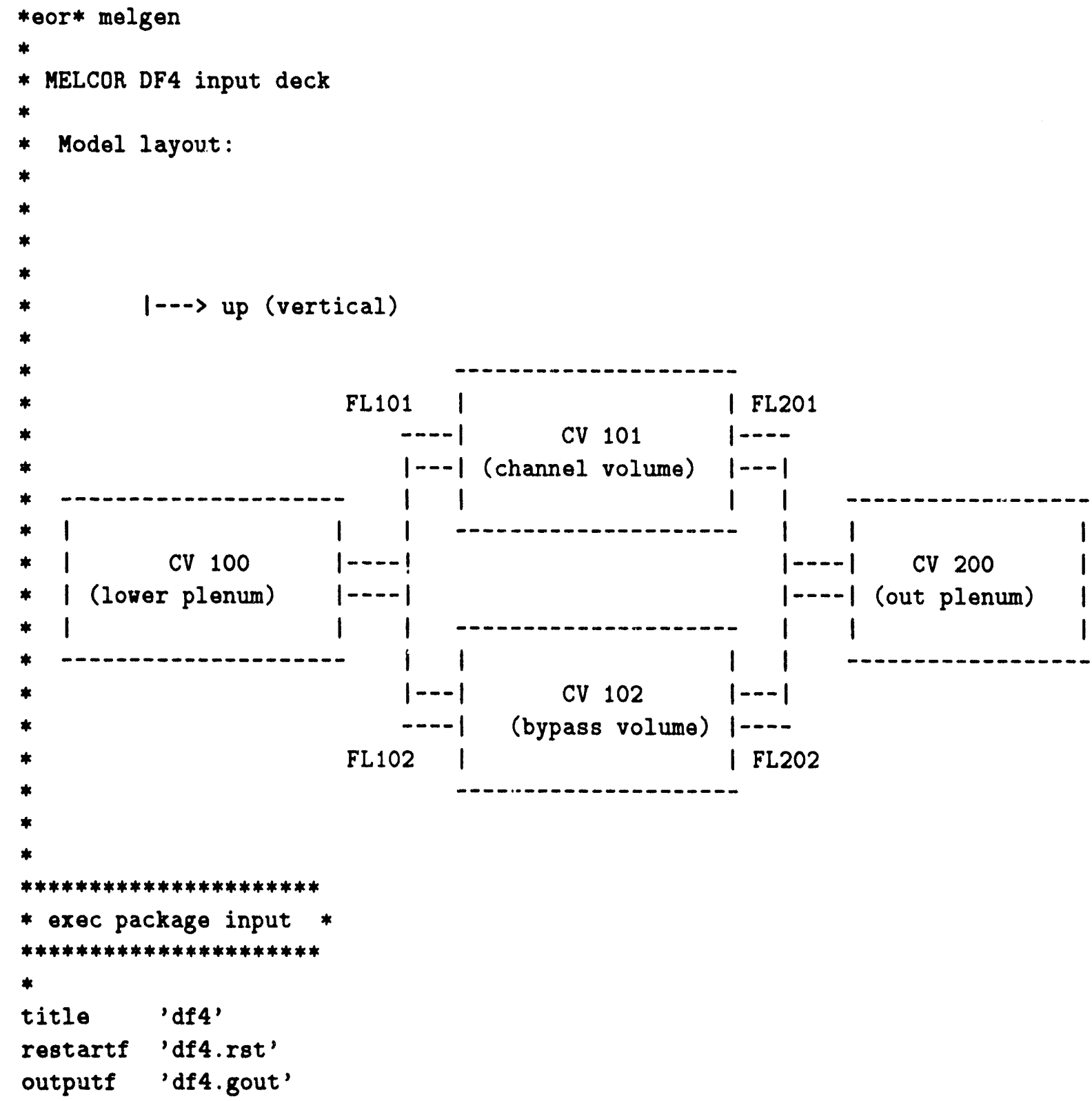




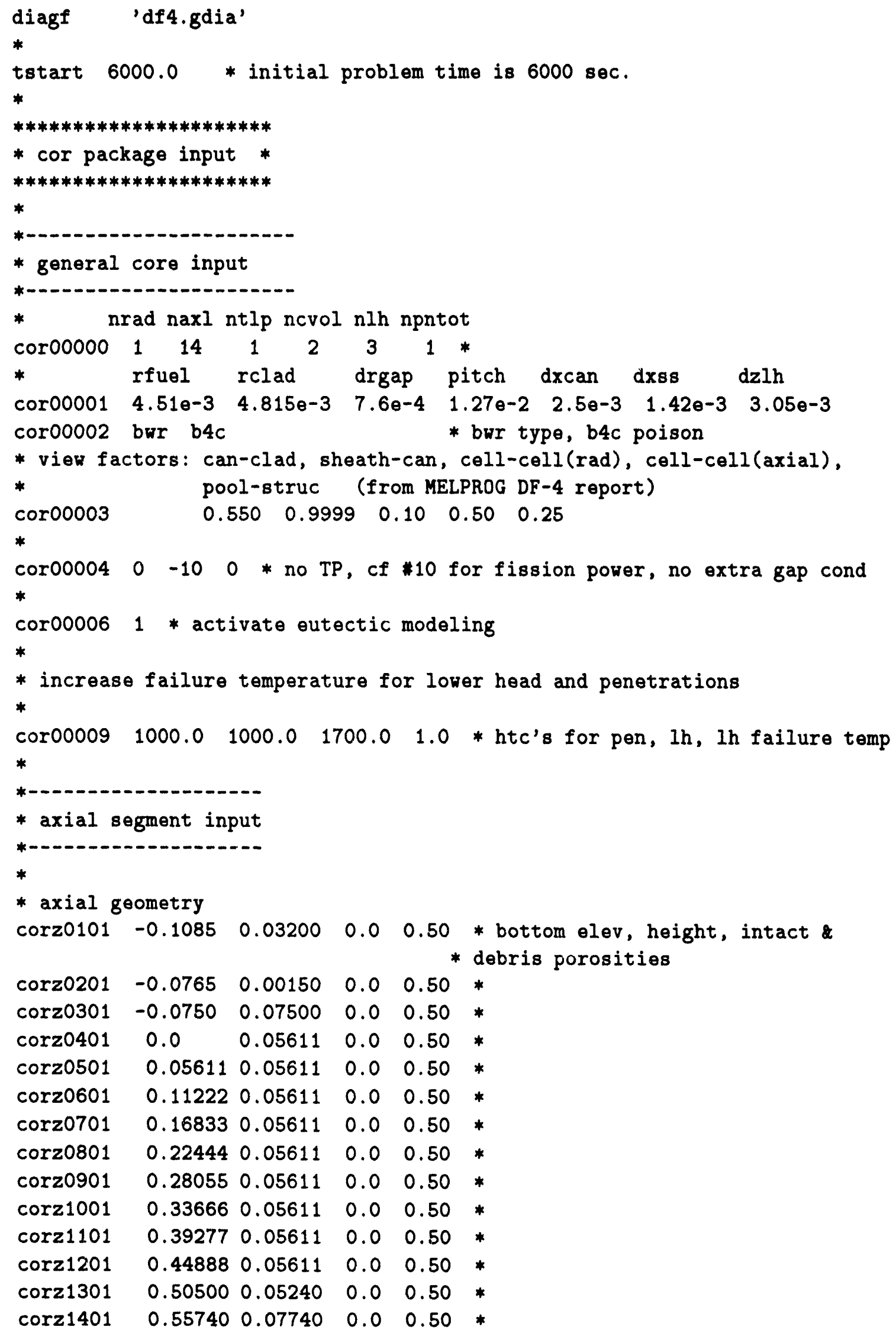




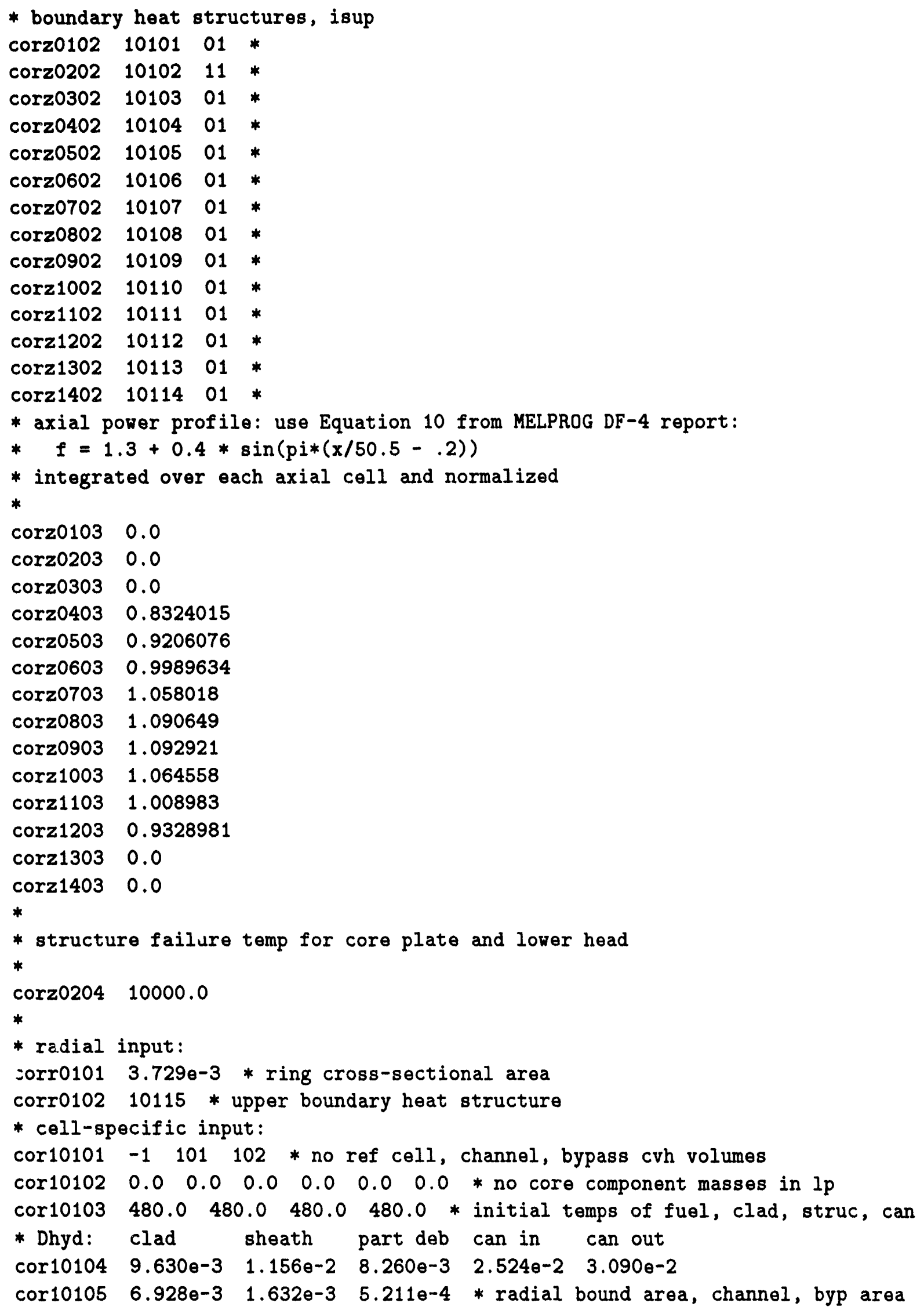




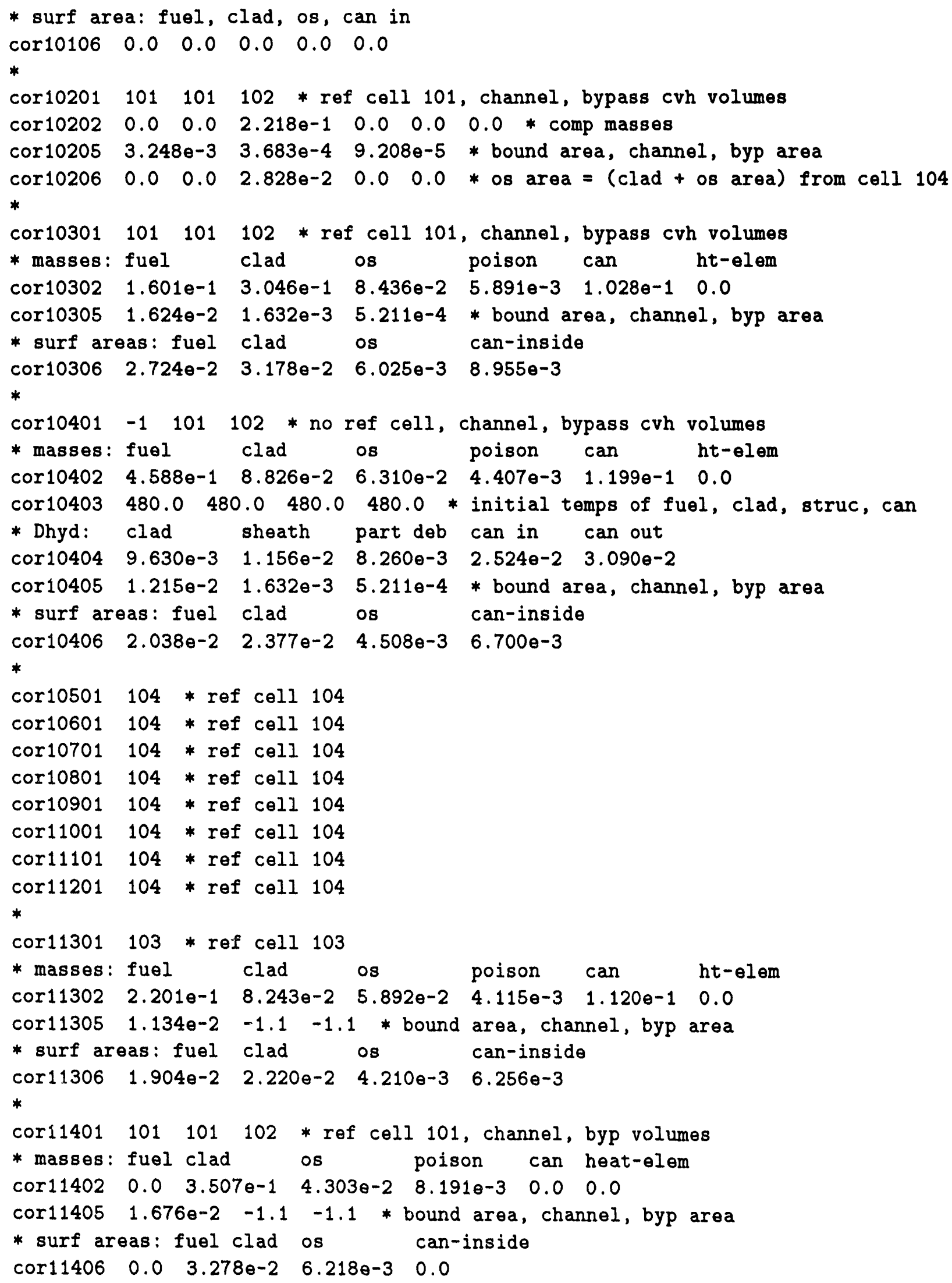




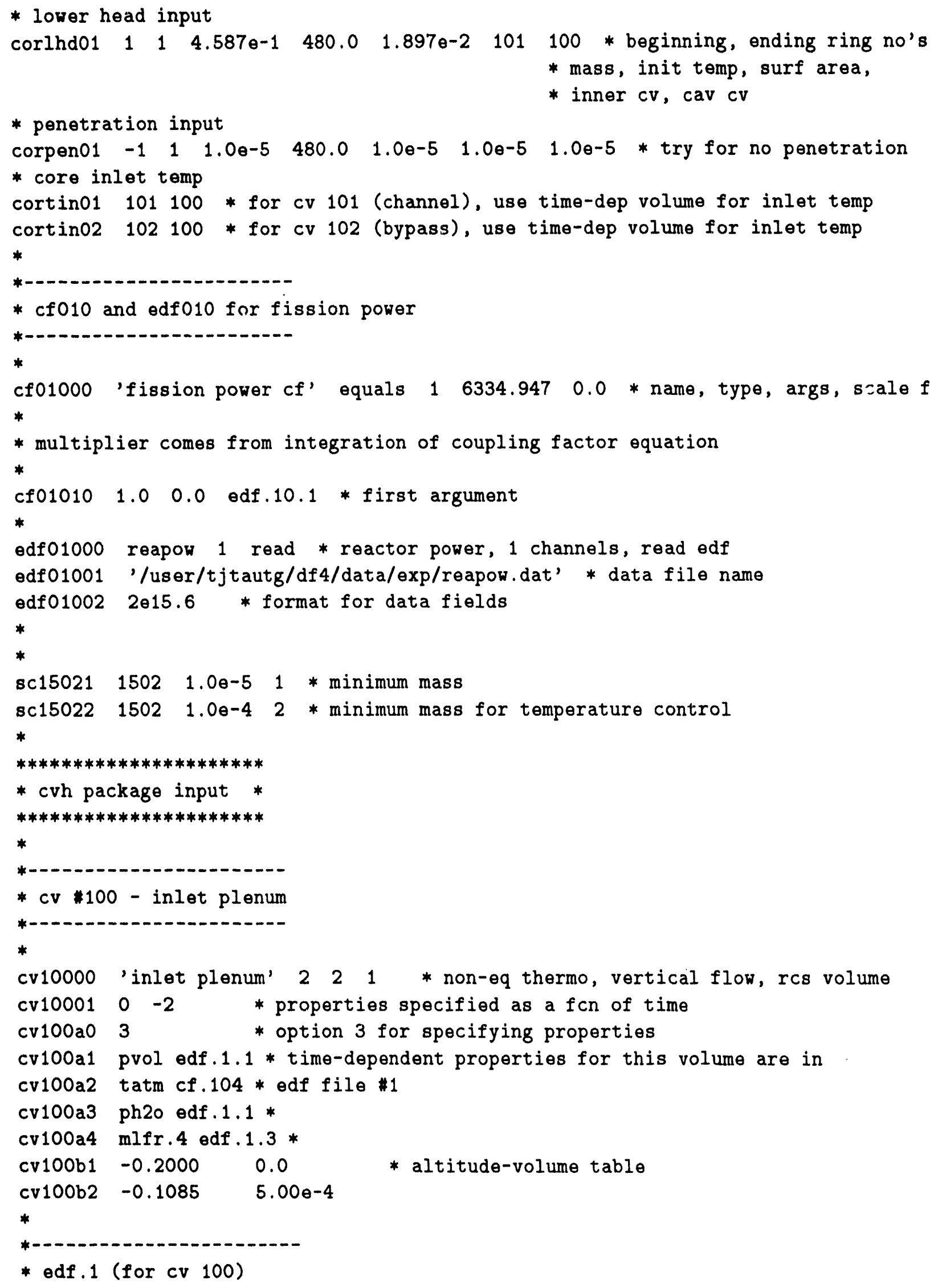




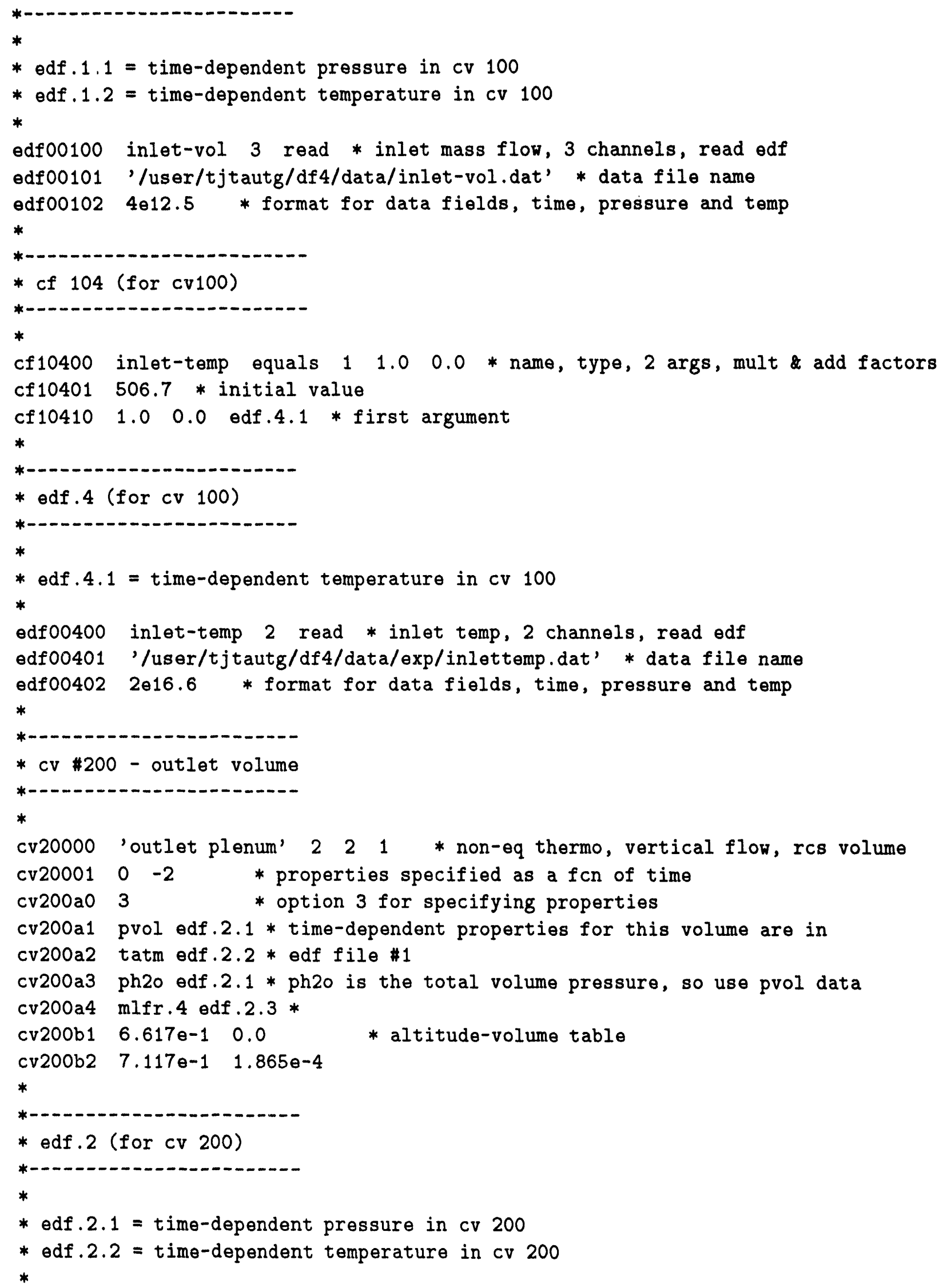




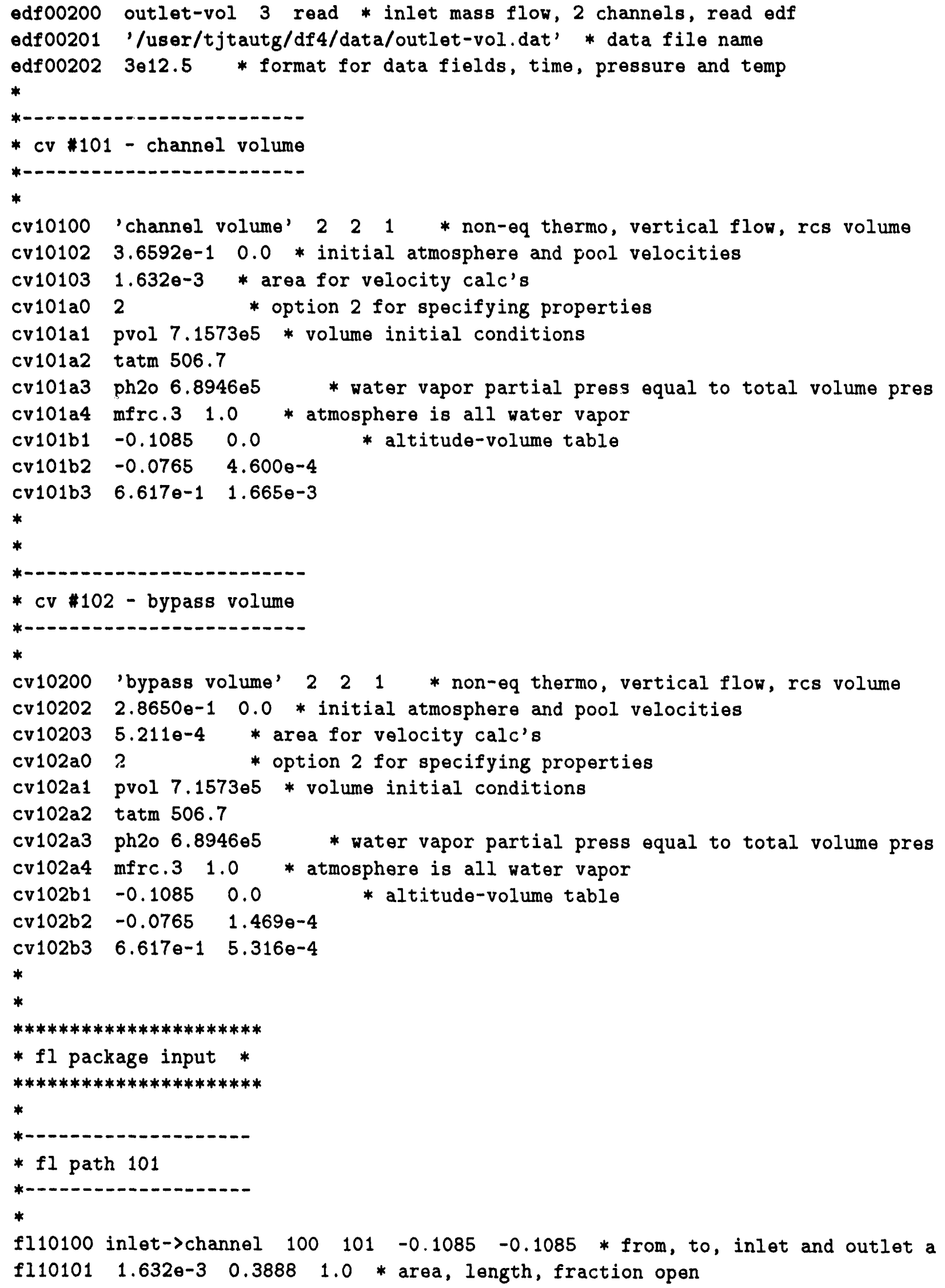




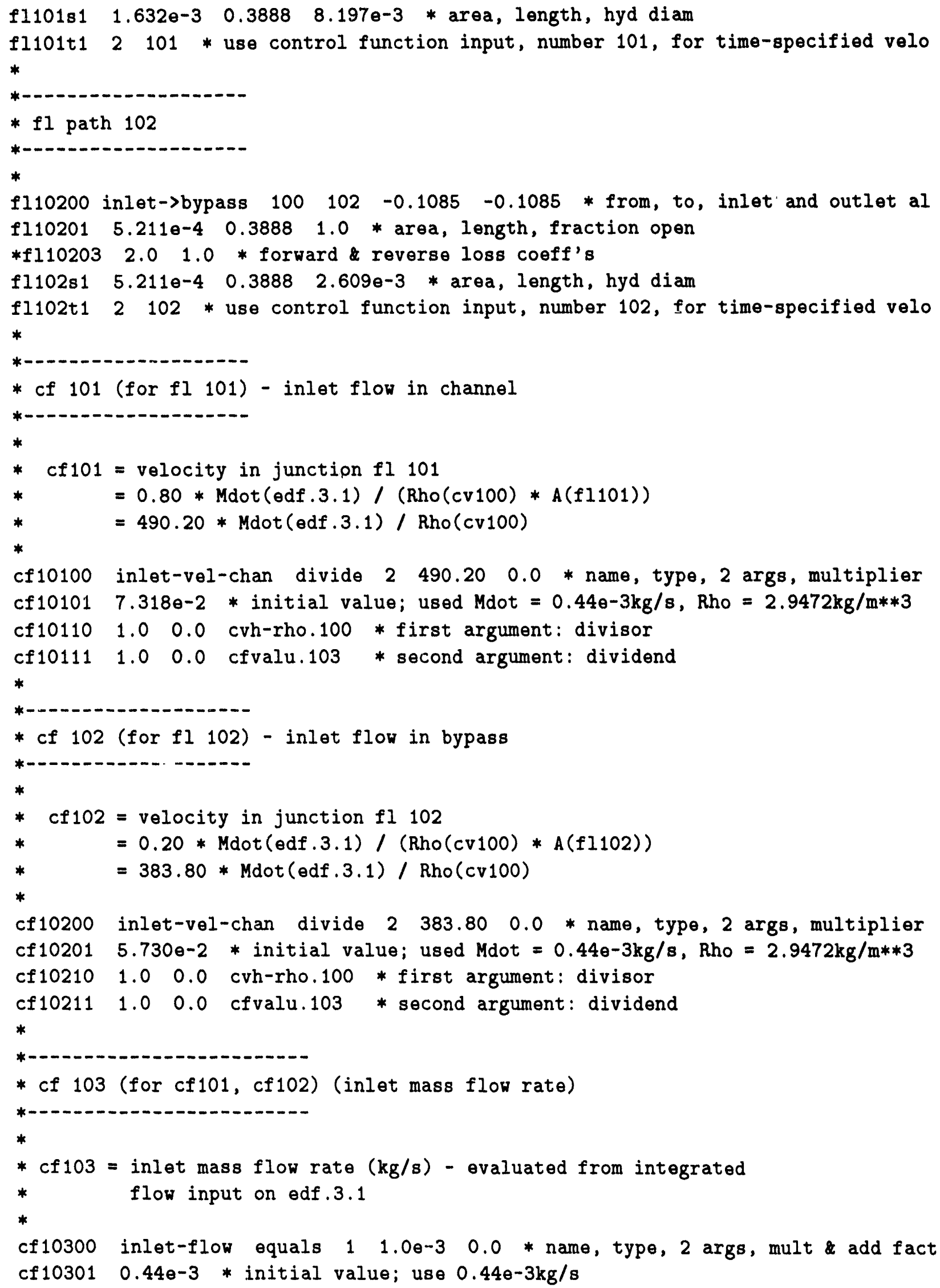




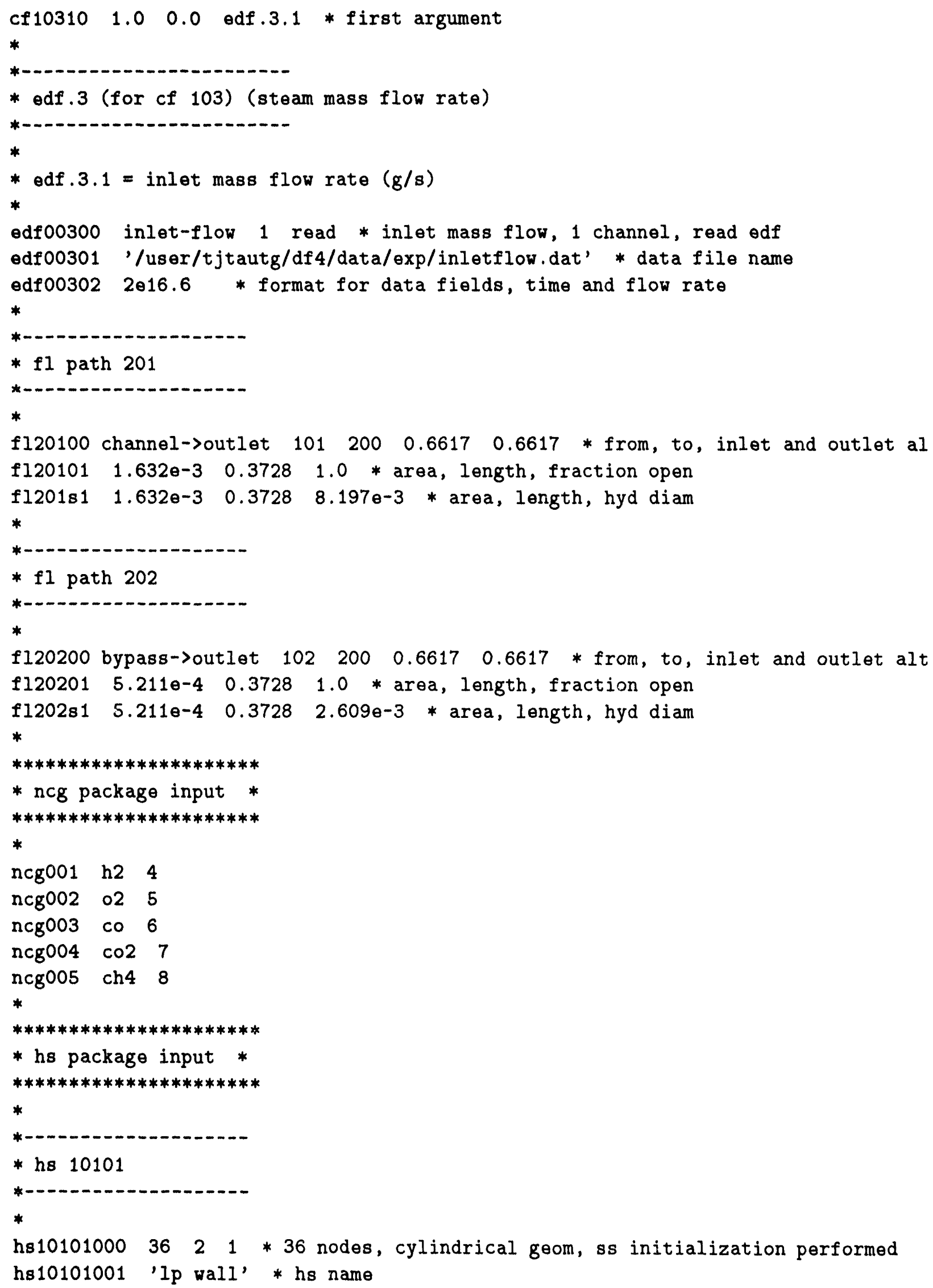




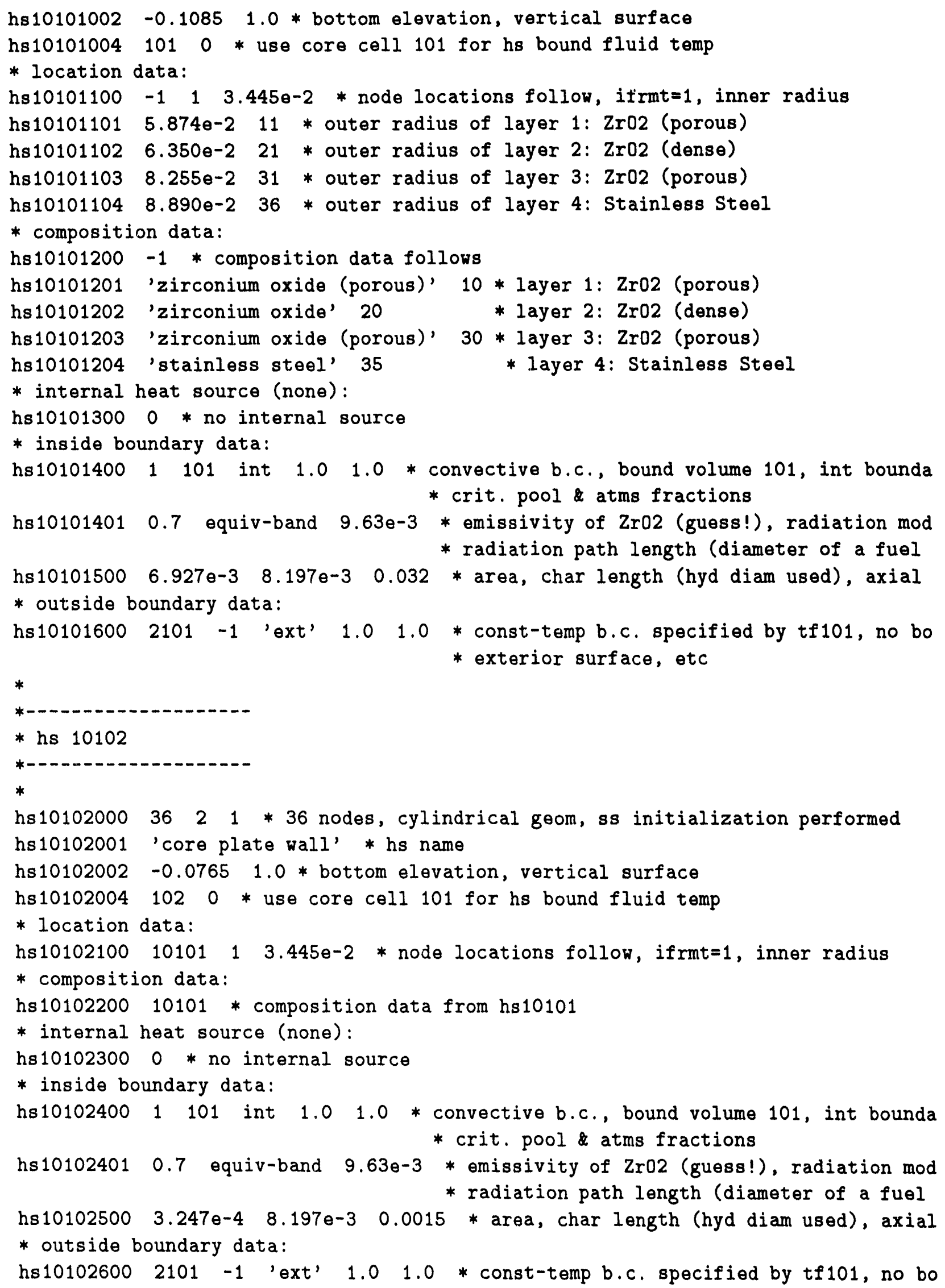


* exterior surface, etc

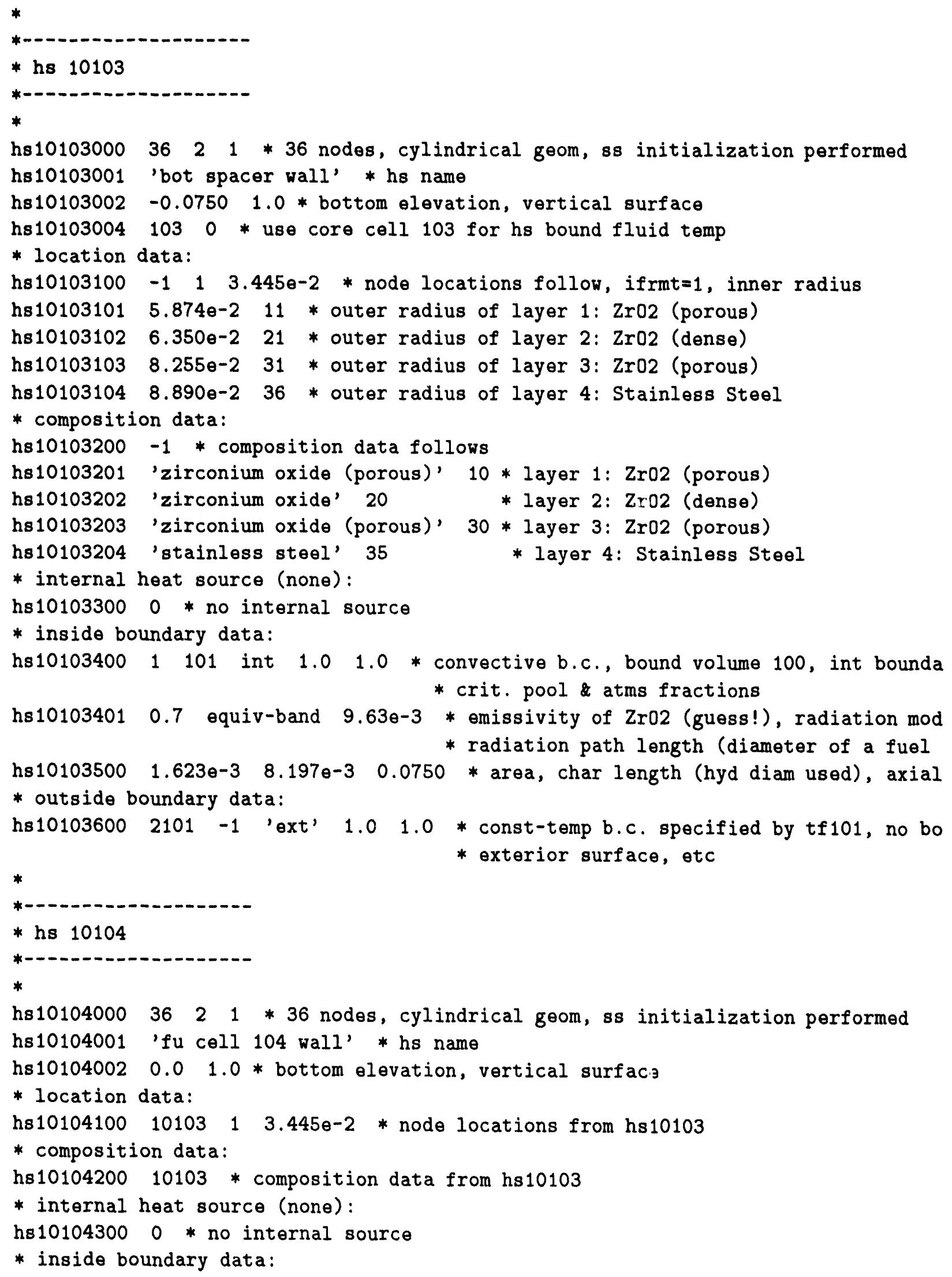


hs10104400 1101 int 1.01 .0 * convective b.c., bound volume 101, int bounda * crit. pool \& atms fractions

hs10104401 0.7 equiv-band $9.63 e-3 *$ emissivity of $\mathrm{Zr02}$ (guess!), radiation mod * radiation path length (diameter of a fuel

hs10104500 1.215e-2 8.197e-3 0.05611 * area, char length (hyd diam used), axia * outside boundary data:

hs10104600 $2101-1$ 'ext' 1.01 .0 * const-temp b.c. specified by tf101, no bo * exterior surface, etc

*

* hs's 10105-10112

* hsmmmmm000:

hs10105000 $36221 * 36$ nodes, cylindrical geom, ss initialization performed hs10106000 $36 \quad 2 \quad 1 * 36$ nodes, cylindrical geom, ss initialization performed hs $10107000 \quad 36 \quad 2 \quad 1 * 36$ nodes, cylindrical geom, ss initialization performed hs $10108000 \quad 36 \quad 2 \quad 1 * 36$ nodes, cylindrical geom, ss initialization performed hs10109000 $36 \quad 2 \quad 1 * 36$ nodes, cylindrical geom, ss initialization performed hs $10110000 \quad 36 \quad 2 \quad 1$ * 36 nodes, cylindrical geom, ss initialization performed hs $1011100036 \quad 2 \quad 1 * 36$ nodes, cylindrical geom, ss initialization performed hs $1011200036 \quad 21 * 36$ nodes, cylindrical geom, ss initialization performed * hsmmmmm001:

hs 10105001 'fu cell 105 wall' * hs name

hs 10106001 'fu cell 106 wall' * hs name

hs 10107001 'fu cell 107 wall' * hs name

hs 10108001 'fu cell 108 wall' * hs name

hs 10109001 'fu cell 109 wall' * hs name

hs 10110001 'fu cell 110 wall' * hs name

hs10111001 'fu cell 111 wall' * hs name

hs10112001 'fu cell 112 wall' * hs name

* hsmmmmm002:

hs10105002 $0.05611 \quad 1.0 *$ bottom elevation, vertical surface

hs 101060020.1122 ? $1.0 *$ bottom elevation, vertical surface

hs10107002 0.1683 . $1.0 *$ bottom elevation, vertical surface

hs $101080020.224441 .0 *$ bottom elevation, vertical surface

hs $101090020.280551 .0 *$ bottom elevation, vertical surface

hs $101100020.336661 .0 *$ bottom elevation, vertical surface

hs $101110020.392771 .0 *$ bottom elevation, vertical surface

hs $101120020.448881 .0 *$ bottom elevation, vertical surface

* hsmmmmm 100:

hs $10105100 \quad 10103 \quad 1 \quad 3.4450-2$ * get node location data from hs 10103

hs $10106100 \quad 10103 \quad 1 \quad 3.445 e-2$ * get node location data from hs 10103

hs $10107100 \quad 10103 \quad 1 \quad 3.445 e-2$ * get node location data from hs 10103

hs $101081001010313.445 e-2$ * get node location data from hs 10103

hs $1010910010103 \quad 1 \quad 3.445 \theta-2$ * get node location data from hs 10103

hs $1011010010103 \quad 1 \quad 3.445 e-2$ * get node location data from hs 10103

hs $1011110010103 \quad 1 \quad 3.445 \theta-2$ * get node location data from hs 10103

hs $1011210010103 \quad 1 \quad 3.445 e-2$ * get node location data from hs 10103 


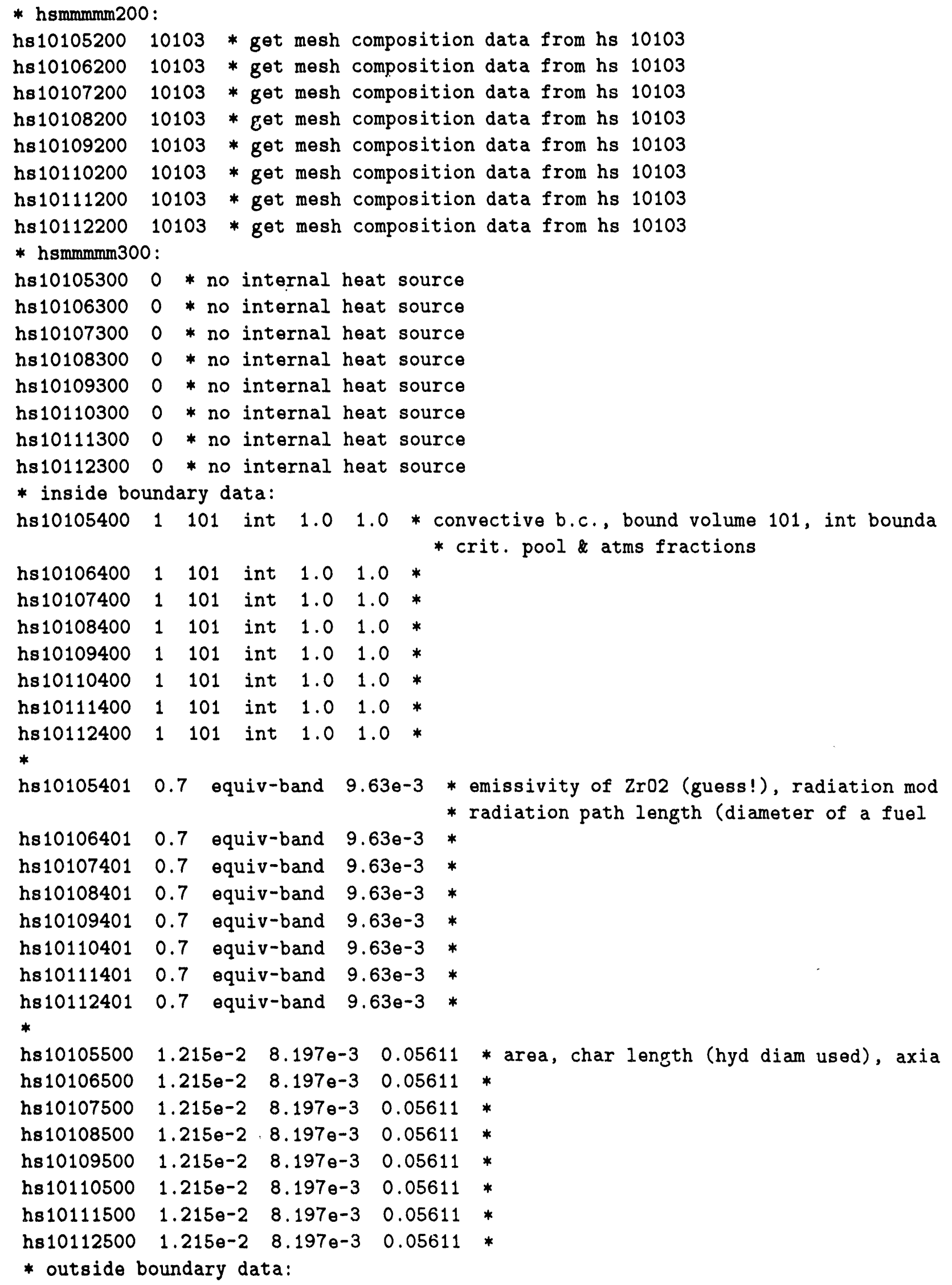




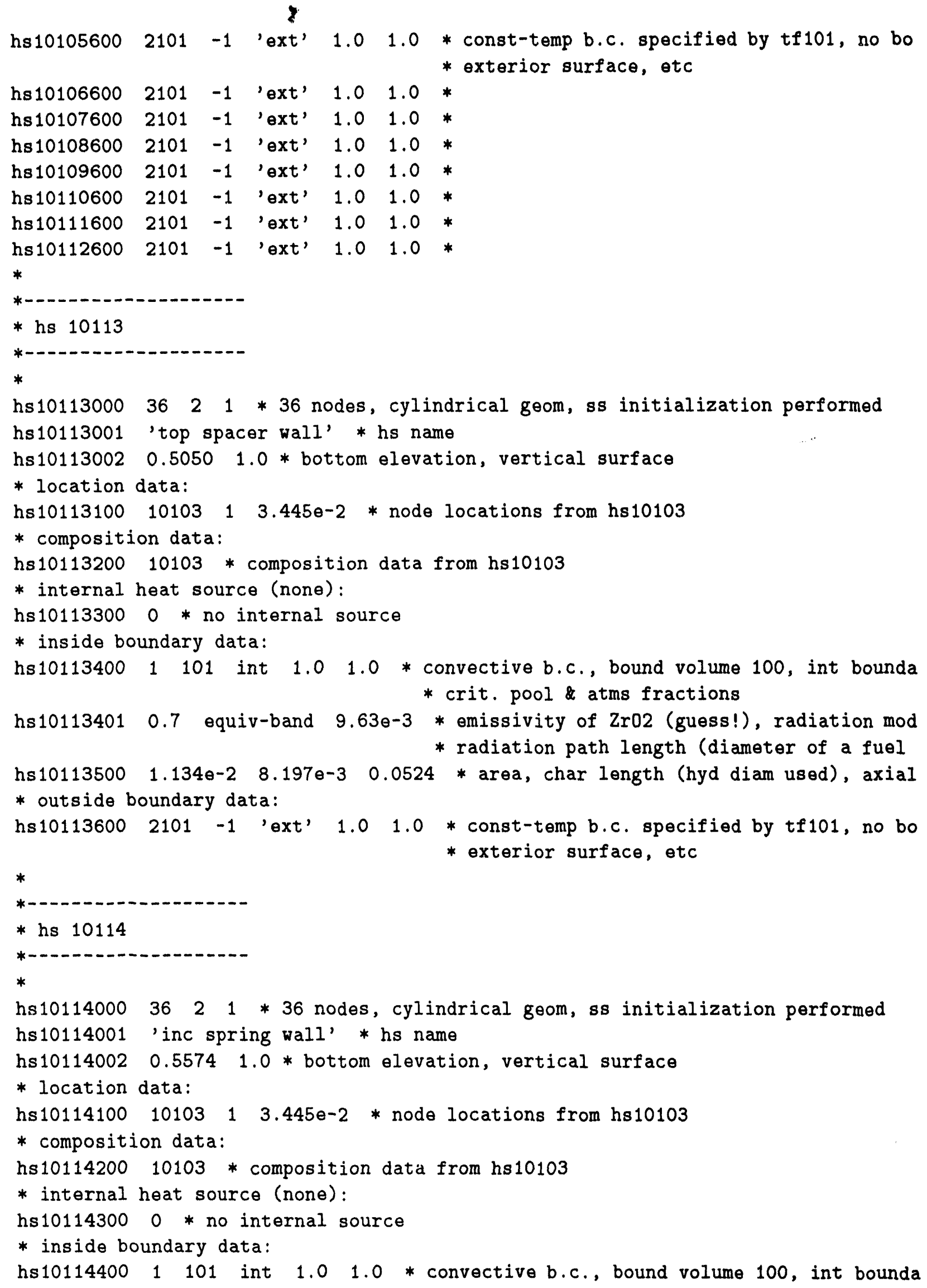


* crit. pool atms fractions

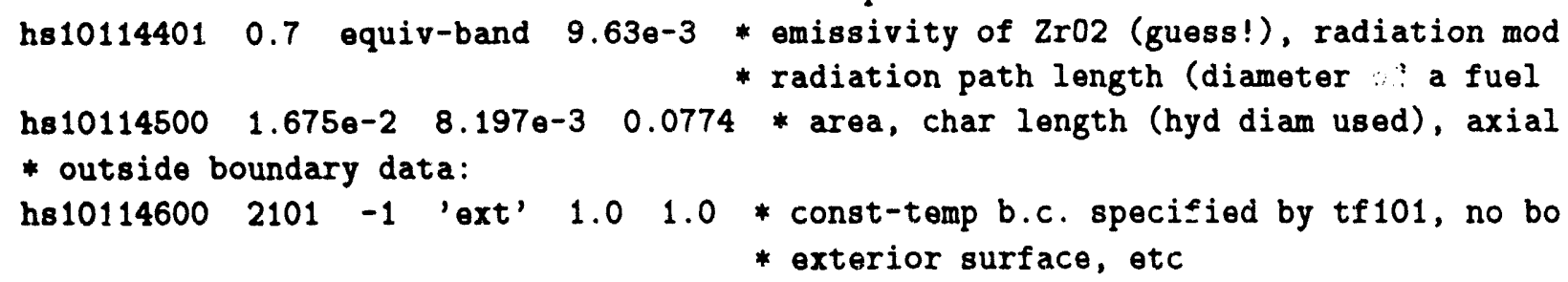

* add boundary fluid temperaturo option to make hs's see core cell

* atm temperatures$$
\text { hs10101004 } 1010
$$

hs $10102004 \quad 102 \quad 0$

hs $10103004 \quad 103 \quad 0$

hs $10104004 \quad 104 \quad 0$

hs $10105004 \quad 105 \quad 0$

hs $10106004 \quad 106 \quad 0$

hs $10107004 \quad 107 \quad 0$

hs $10108004 \quad 108 \quad 0$

hs $10109004 \quad 109 \quad 0$

hs $10110004 \quad 110 \quad 0$

hs10111004 1110

hs10112004 1120 


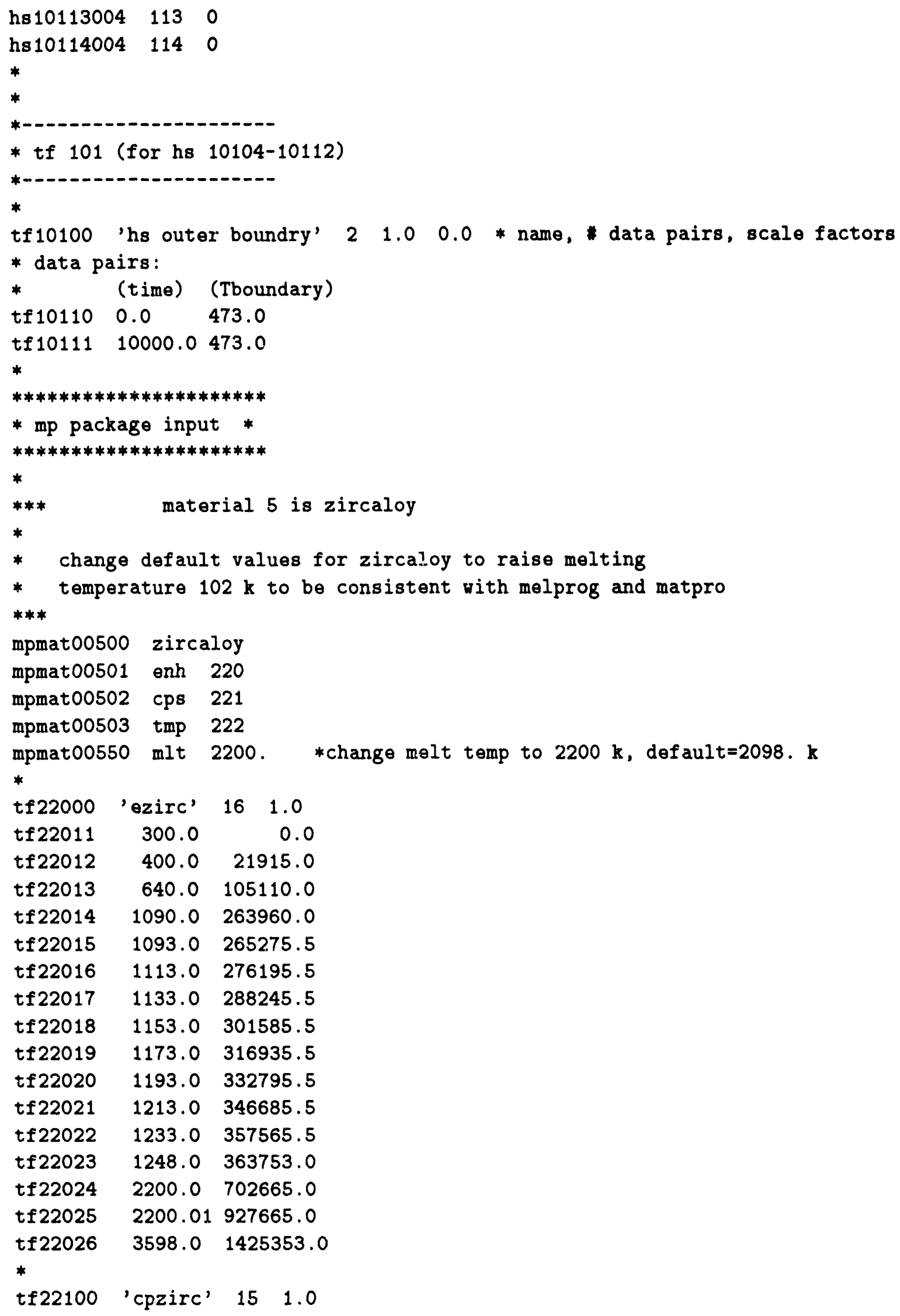




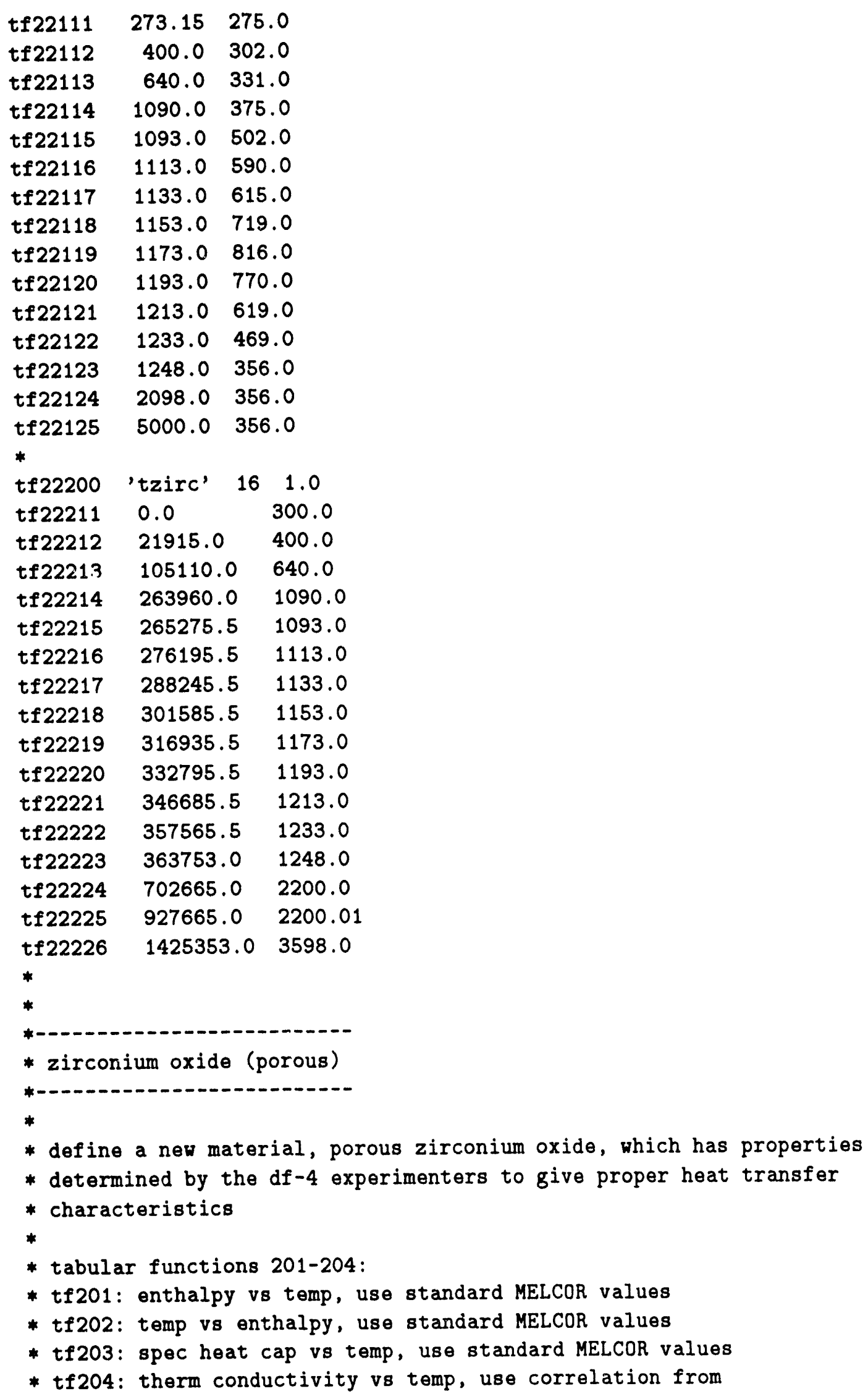




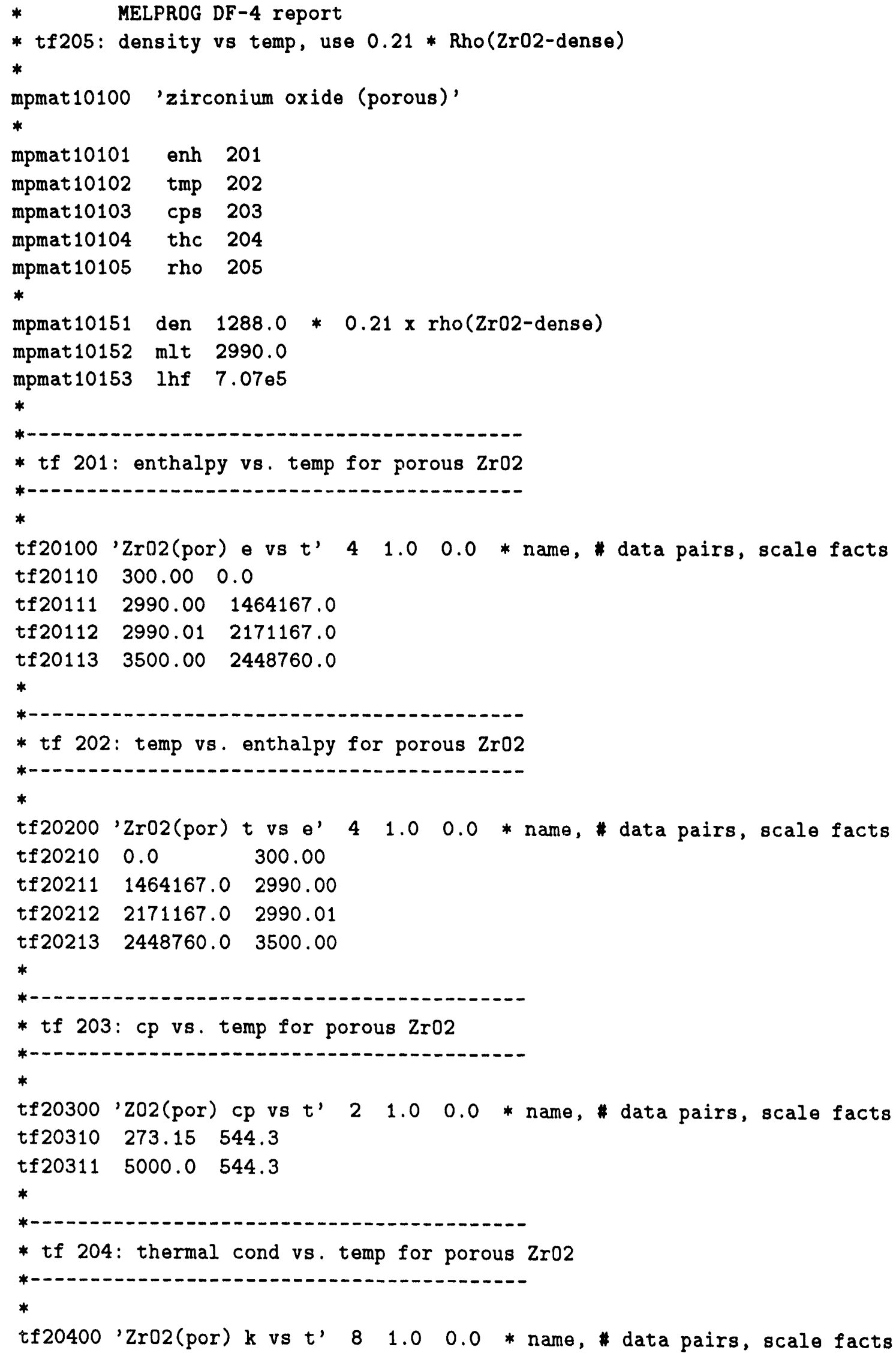




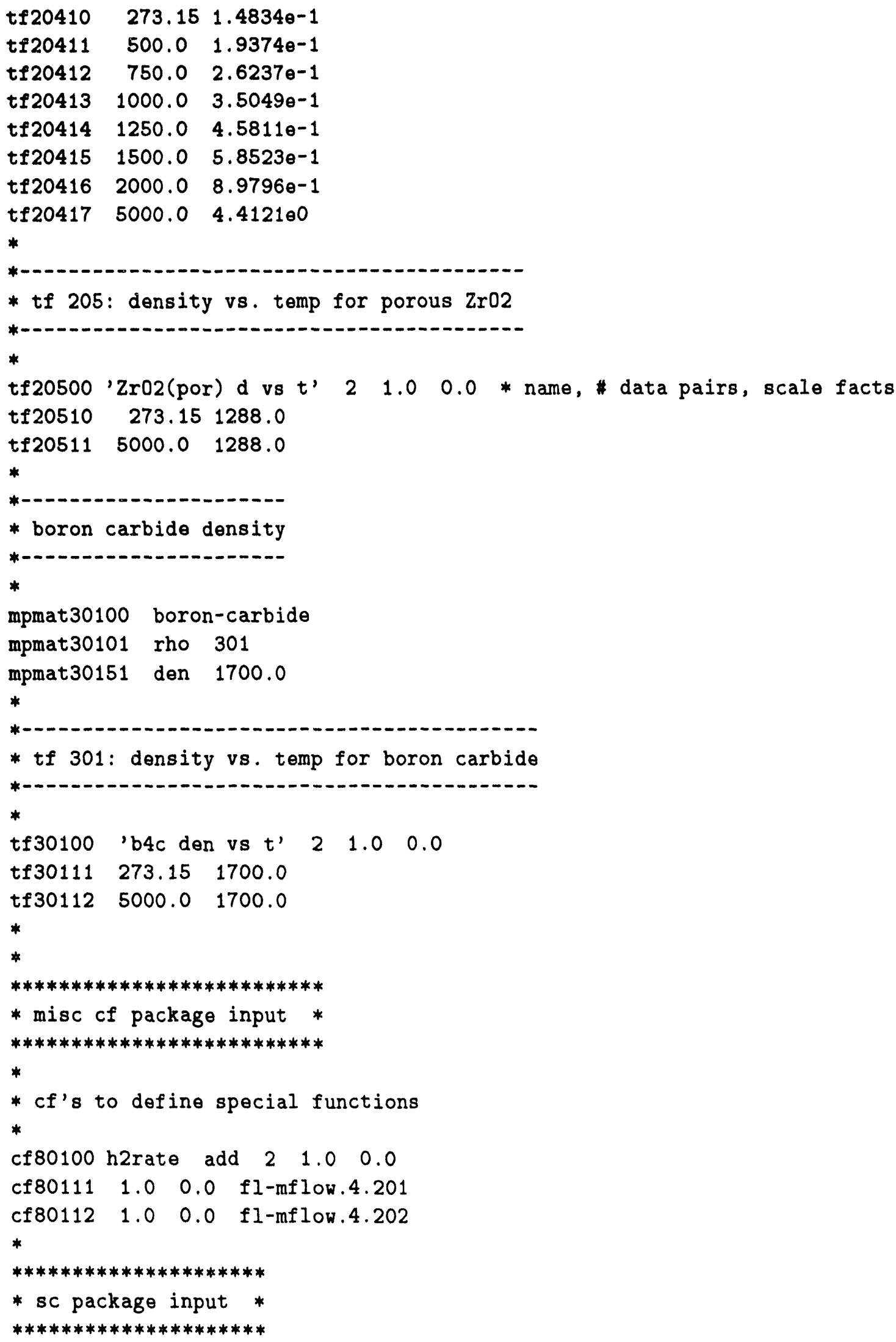




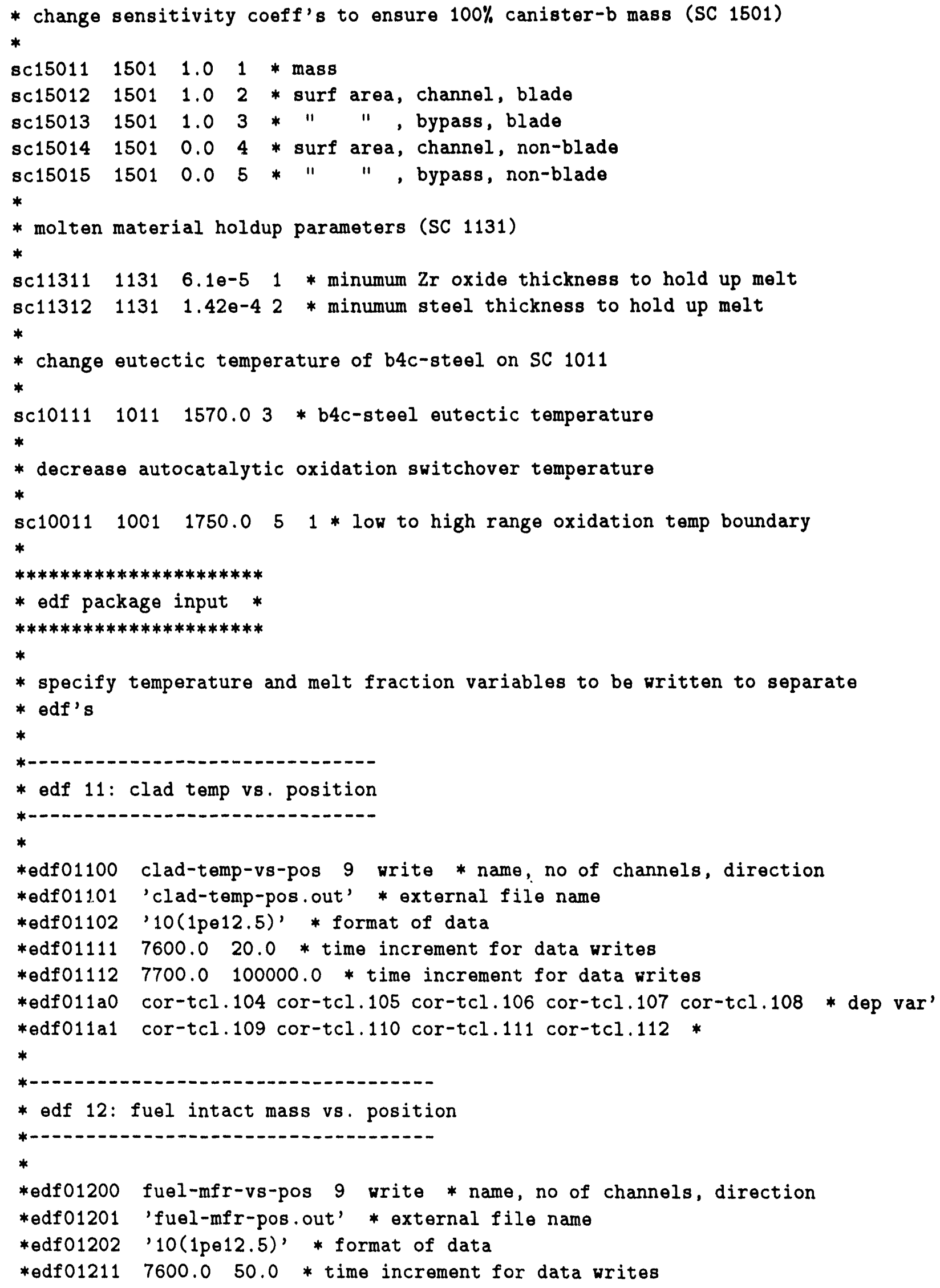




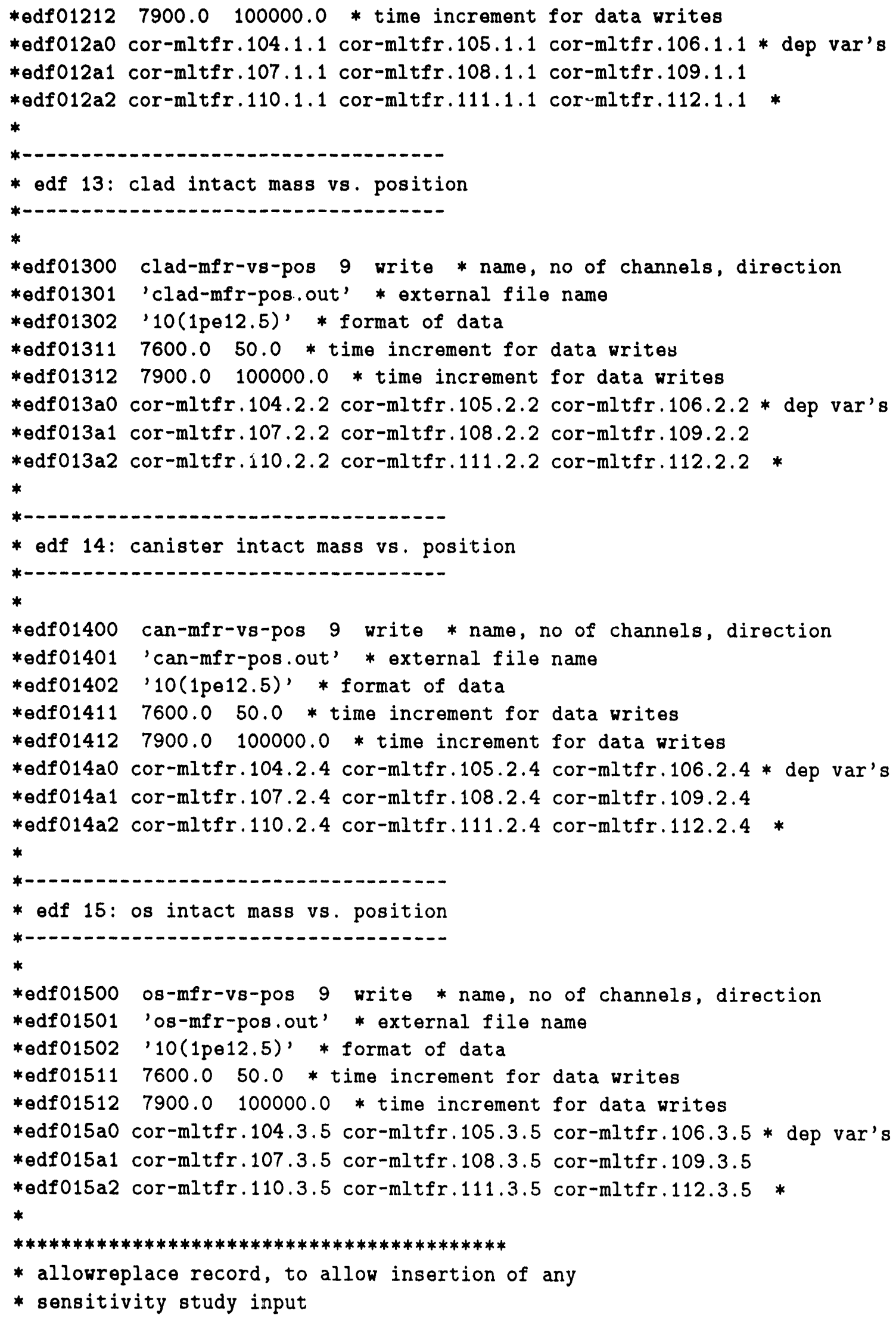




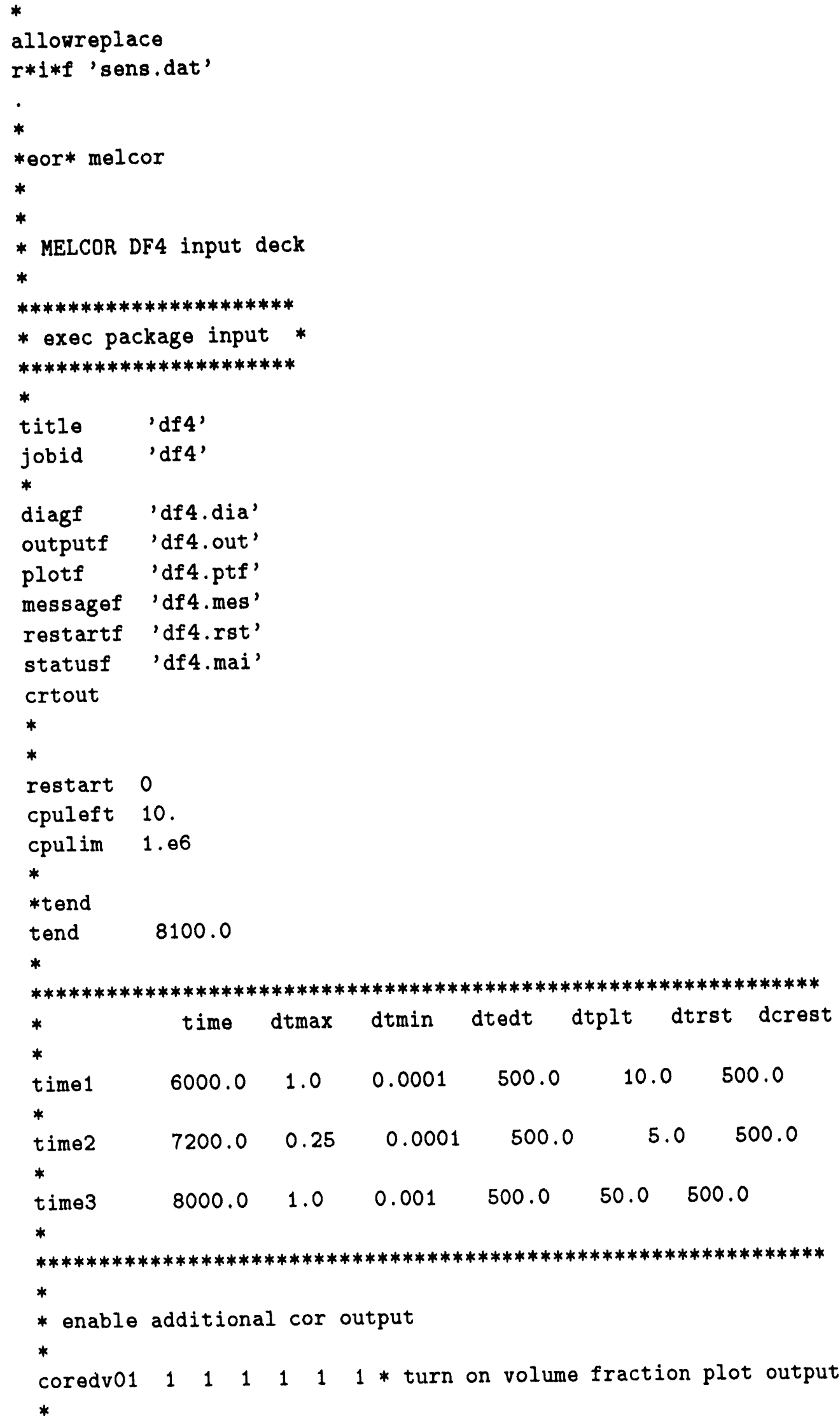




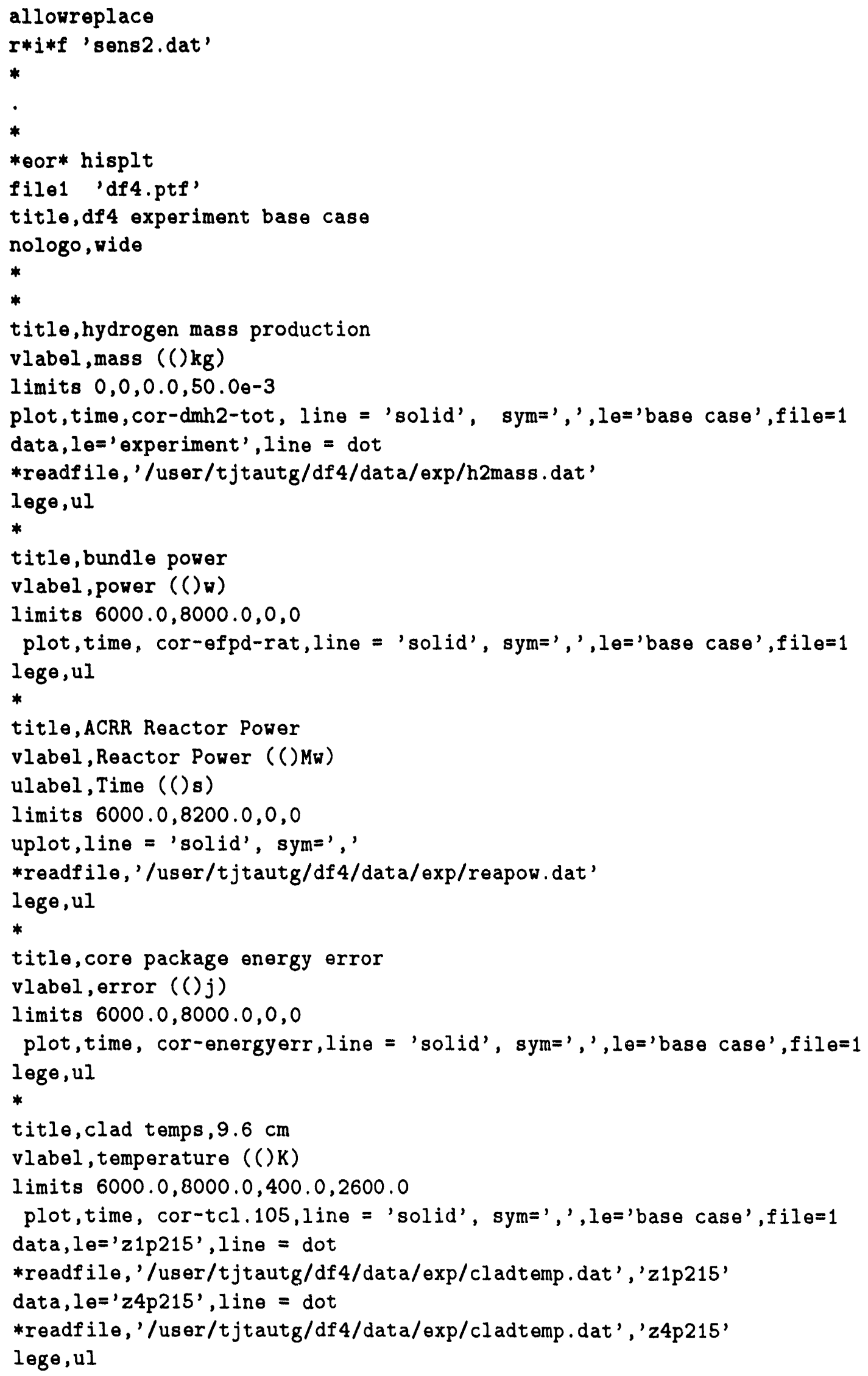




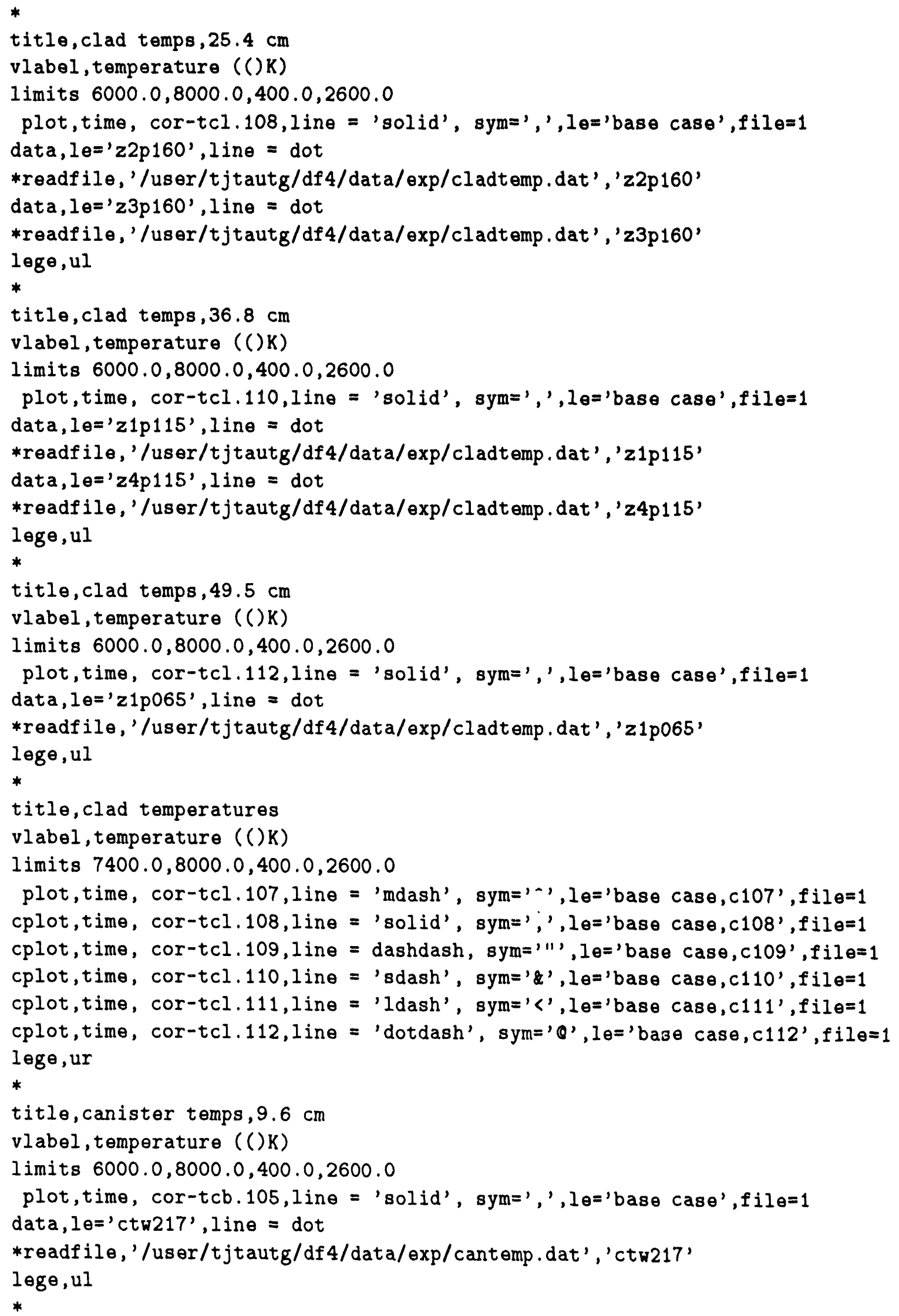


title,canister temps, $25.4 \mathrm{~cm}$

vlabel, temperature ( ( ) K)

limits $6000.0,8000.0,400.0,2600.0$

plot,time, cor-tcb.108,line = 'solid', sym=', ', le='base case', file=1

data, $1 \theta=' \operatorname{ctw162}$, line $=\operatorname{dot}$

*readfile, '/user/tjtautg/df4/data/exp/cantemp.dat' , 'ctw162'

data, $10=$ 'cbw162', line $=\operatorname{dot}$

*readfile, '/user/tjtautg/df4/data/exp/cantemp. dat' , 'cbw162'

lege, ul

*

title,canister temps, $36.8 \mathrm{~cm}$

vlabel, temperature (()K)

limits $6000.0,8000.0,400.0,2600.0$

plot, time, cor-tcb.110,1ine = 'solid', sym=', ', le='base case', file=1

data, le ='ctw117', line = dot

*readfile, '/user/tjtautg/df4/data/exp/cantemp.dat' , 'ctw117'

data, $10=$ ' cbw117', line $=\operatorname{dot}$

*readfile, '/user/tjtautg/df4/data/exp/cantemp.dat' , 'cbw117'

lege, ul

*

title,canister temps, $49.5 \mathrm{~cm}$

vlabel, temperature (()K)

limits $6000.0,8000.0,400.0,2600.0$

plot,time, cor-tcb.112,1ine = 'solid', sym=', ', le='base case', file=1

data, le ='ctw067', line $=\operatorname{dot}$

*readfile, '/user/tjtautg/df4/data/exp/cantemp.dat' , 'ctw067'

lege,ul

*

title, canister temperatures (high)

vlabel, temperature $(() \mathrm{K})$

limits $7400.0,8000.0,400.0,2600.0$

plot,time, cor-tcb.108,line = 'solid', sym=', ', le='base case,c108', file=1

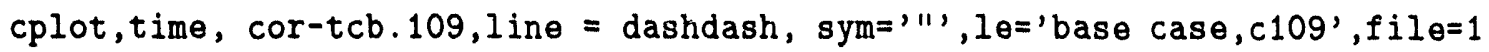

cplot,time, cor-tcb.110,line = 'sdash', sym=' $\&$ ', le $=^{\prime}$ base case, $c 110^{\prime}$, fil $\theta=1$

cplot, time, cor-tcb.111, line = 'ldash', sym=' $\langle$ ', le='base case,c111', file=1

cplot, time, cor-tcb.112,line = 'dotdash', sym=' $\theta^{\prime}, l_{\theta}=$ 'base $^{\prime}$ case, $c 112^{\prime}, f i l e=1$

$\operatorname{lege}, \mathrm{lr}$

*

title, canister temperatures (low)

vlabel, temperature (()K)

limits $7400.0,8000.0,400.0,2600.0$

plot, time, cor-tcb.104,line = 'mdash', sym=' ' ', le='base case, c107', fil $\theta=1$

cplot,time, cor-tcb.105, line = 'solid', sym=', ', le='base case,c108', file=1

cplot, time, cor-tcb.106, line = dashdash, $s y m=\prime \prime \prime \prime, 1 \theta='$ base case, c109', file=1

cplot, time, cor-tcb.107,line = 'sdash', sym=' $\&$ ', le='base case, $c 110$ ', file=1

$\operatorname{leg} \theta, \mathrm{lr}$

title, intact $\mathrm{zr}$ mass in clad 


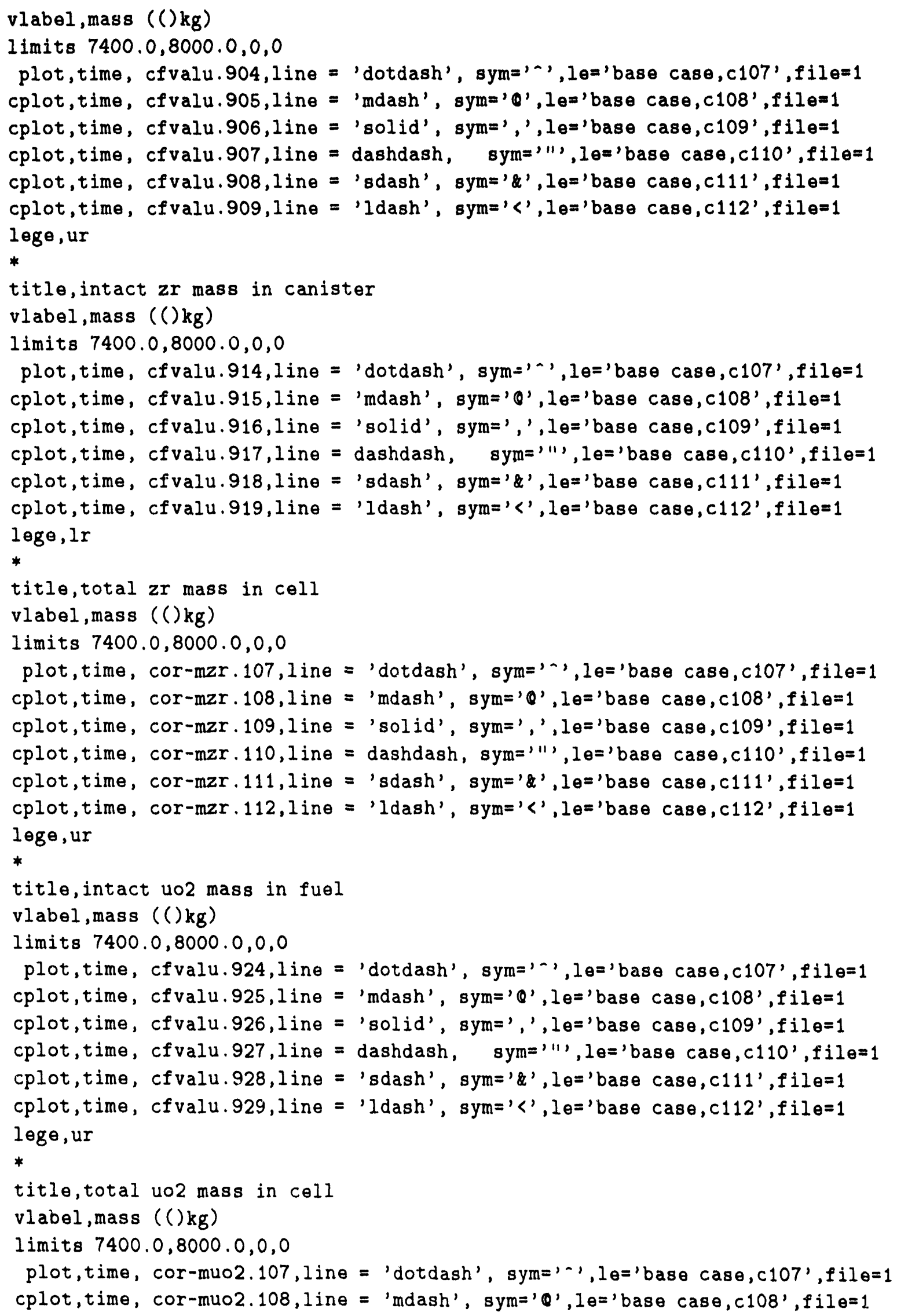




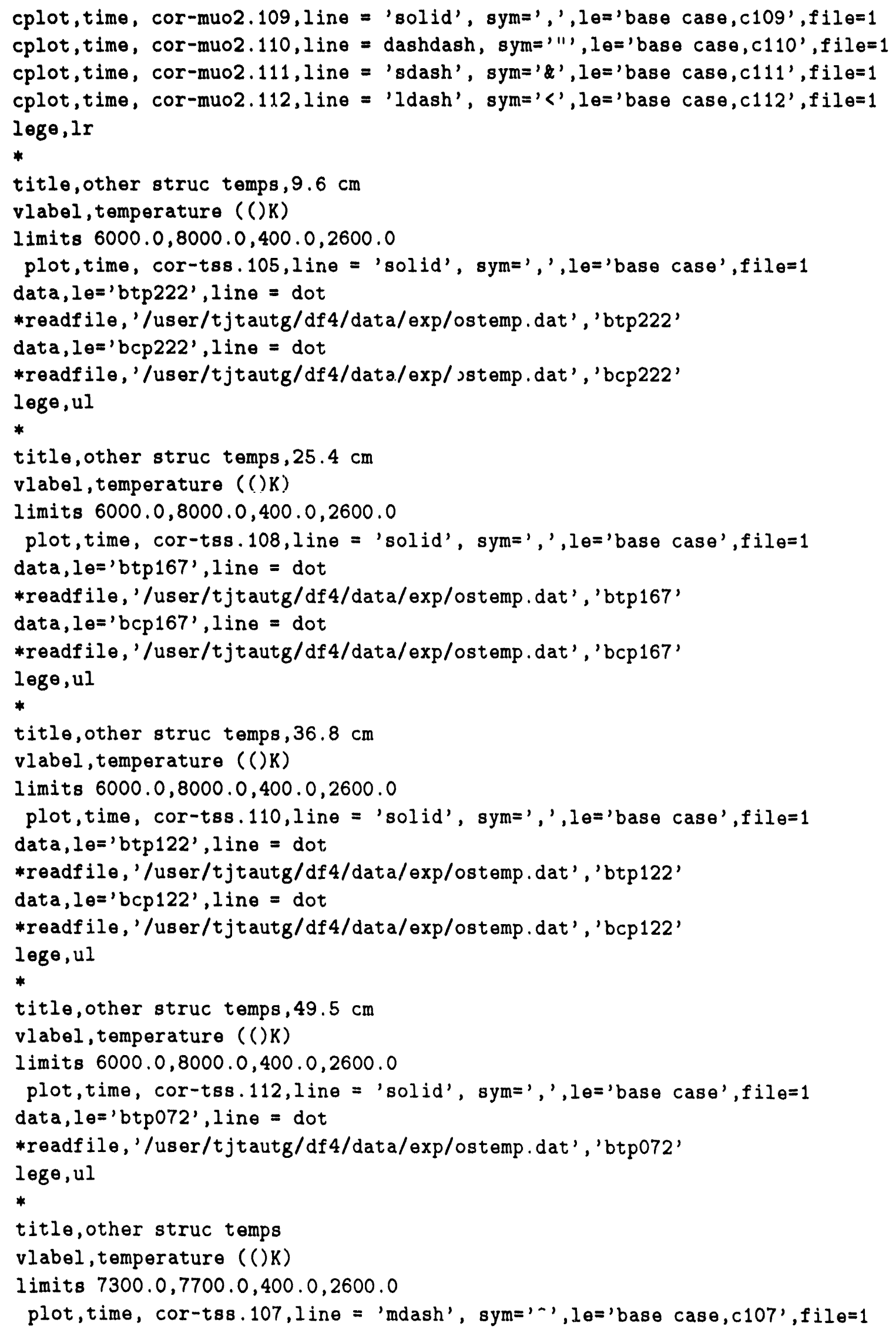


cplot,time, cor-tss.108, line = 'solid', sym=', ', le='base case,c108', file=1 cplot,time, cor-tss.109,line = dashdash, sym='"', le='base case,c109', fil $\theta=1$ cplot, time, cor-tss.110,line = 'sdash', sym=' $a$ ', le='base case,c110',file=1

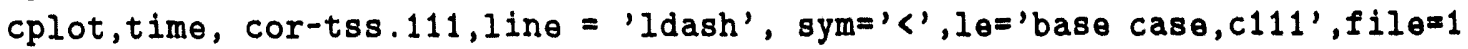

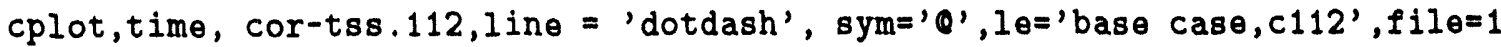
data, le ='btp167', line $=\operatorname{dot}$ *readfile, '/user/tjtautg/df4/data/exp/ostemp.dat', 'btp167' data, $1 \theta=$ 'bcp 167 ', line $=\operatorname{dot}$ *readfile, '/user/tjtautg/df4/data/exp/ostemp.dat', 'bcp167' data, le ='btp122', line $=\operatorname{dot}$ *readfile, '/user/tjtautg/df4/data/exp/ostemp.dat' , 'btp122' data, $1 \theta=$ 'bcp122', line = dot *readfile, '/user/tjtautg/df4/data/exp/ostemp.dat', 'bcp122' data, $10=$ 'btp072', line $=\operatorname{dot}$ *readfile, '/user/tjtautg/df4/data/exp/ostemp.dat' , 'btp072'

lege, next

title, intact steel mass in os

vlabel, mass $(() \mathrm{kg})$

limits $7300.0,7700.0,0,0$ plot,time, cfvalu.934,line = 'dotdash', sym='a', le='base case,c107',file=1 cplot,time, cfvalu.935, line = 'mdash', sym=' $\theta$ ', le='base case,c108',fil $\theta=1$ cplot, time, cfvalu.936,line = 'solid', sym=', ', le='base case,c109',file=1

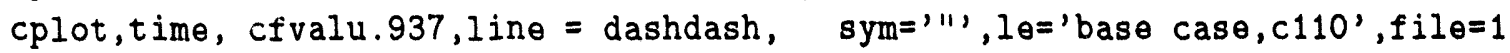
cplot, time, cfvalu. 938,1 ine = 'sdash', sym='\&', le='base case,c111',file=1 cplot, time, cfvalu. 939,1 ine $=$ 'ldash', sym=' $\left\langle\right.$ ', $l_{\theta}=$ 'base case, $c 112$ ', fil $\theta=1$ $\log \theta, 11$

*

title, total steel mass in cell

vlabel, mass $(() \mathrm{kg})$

limits $7300.0,7700.0,0,0$

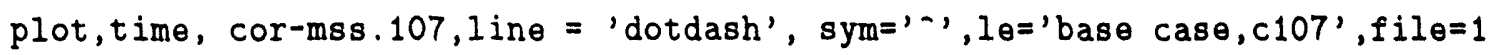
cplot, time, cor-mss. 108,1 ine = 'mdash', sym=' $\theta$ ', le ' $^{\prime}$ base case, $c 108$ ', file=1 cplot, time, cor-mss.109, line = 'solid', sym=',', le='base case,c109', file=1 cplot, time, cor-mss.110, line = dashdash, $s y m='$ '', le ' $^{\prime}$ base case,c110', file=1 cplot,time, cor-mss.111,line = 'sdash', sym=' $q$ ', le='base case,c111', fil $\theta=1$

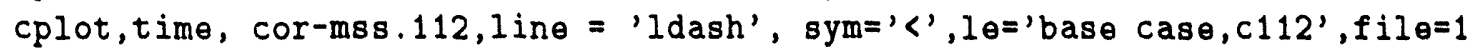
$\operatorname{leg} \theta, u 1$

*

title, intact b4c mass in os

vlabel, mass (() kg)

limits $7300 \cdot 0,7700 \cdot 0,0,0$

plot, time, cfvalu.944,line = 'dotdash', sym=' ', , le='base case,c107', file=1

cplot, time, cfvalu. 945,1 ine $=$ 'mdash', sym=' $\theta^{\prime}, l_{\theta}=$ 'base $^{\prime}$ case, $c 108^{\prime}$, fil $\theta=1$

cplot, time, cfvalu.946, line = 'solid', sym=', ', le='base case,c109', file=1

cplot,time, cfvalu. 947,1 ine $=$ dashdash, sym=''"',le='base case,c110',file=1

cplot,time, cfvalu.948, line = 'sdash', sym=' 2 ', le='base case, c111', file=1

cplot, time, cfvalu.949, line = 'ldash', sym='<', le='base case, $c 112$ ', file=1 


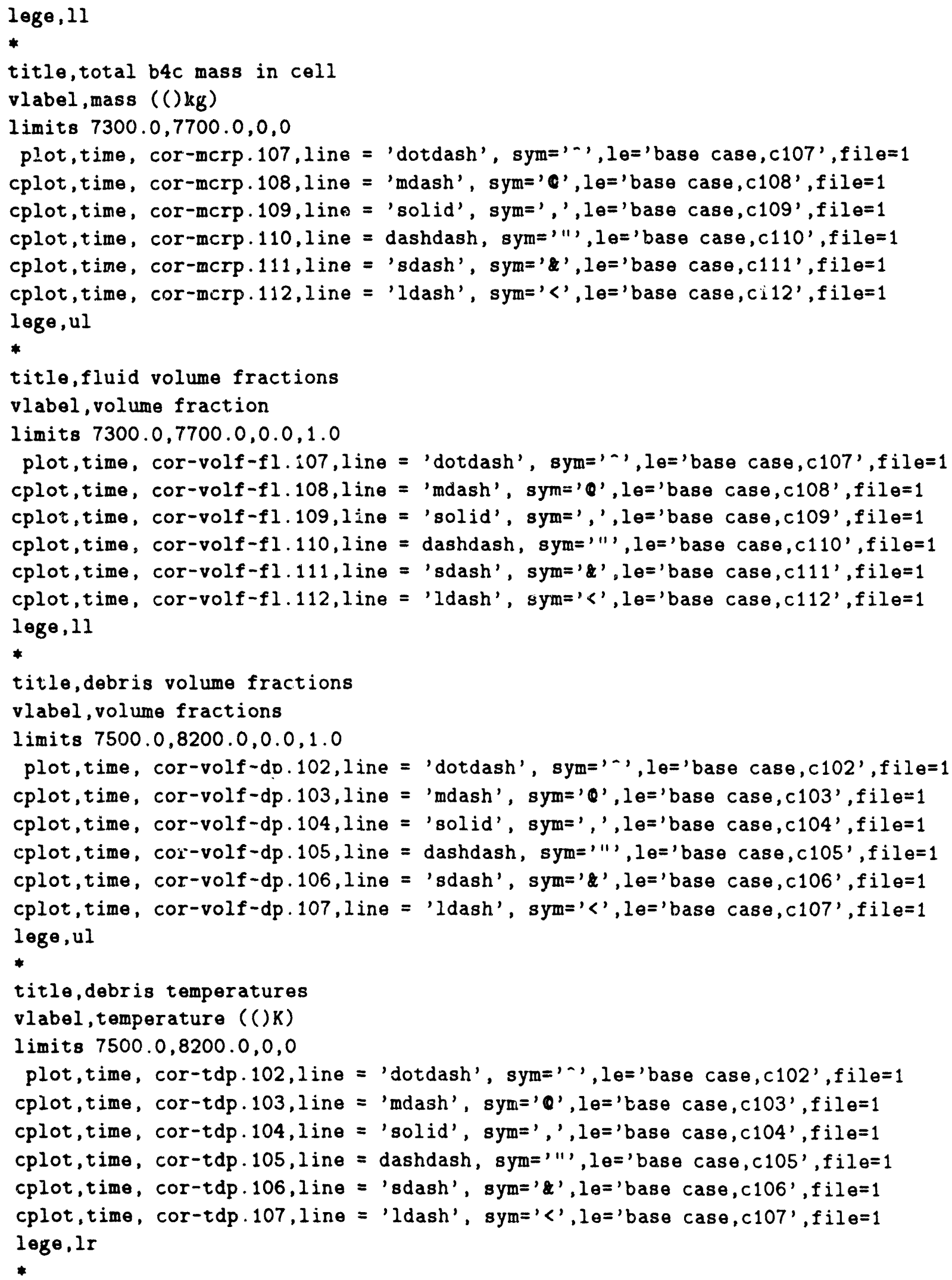


External Distribution:

U. S. Nuclear Regulatory Commission (19)

Attn: S. Acharya, NLS-372

Y. S. Chen, NLN-344

M. A. Cunningham, NLS-372

F. Eltawila, NLN-344

R. B. Foulds, NLN-344

Dr. Sud Basu, NLN-344

C. Gingrich, NLN-344

C. G. Tinkler, NLN-344

B. Wright, NLN-344

L. E. Lancaster, NLS-372

R. O. Meyer, NLS-007

J. A. Mitchell, NLS-314

C. P. Ryder, NLS-372

L. Soffer, NLS-324

B. Sheron, NLS-007

J. A. Murphy, NIS-007

L. M. Shotkin, NLN-353

N. Lauben, NLN-35.3

R. Landry, NLN-344

Washington, DC 20555

S. Y. Then

Argowne National Laboratory

9700 South Cass Avenue

Argonne, IL 60439

Battelle Columbus Laboratories (3)

Attn: P. Cybulskis

M. Carmel

R. S. Denning

$505 \mathrm{King}$ Avenue

Columbus, OH 43201

Brookhaven National Laboratory (2)

Attn: I. K. Madni

T. Pratt

Bldg. 130

32 Lewis

Upton, NY 11973 
Idaho National Engineering Laboratory (5)

Attn: A. Brown

R. J. Dallinan

D. W. Golden

S. E. Reed

G. W. Johnsen

EG\&G Idaho

P. O. Box 1625

Idaho Falls, ID 83404

D. Jones

EI International

P. O. Box 50736

Idaho Falls, ID 83405

Electric Power Research Institute (3)

Attn: E. Fuller

R. N. Oehlberg

B. R. Sehgal

P. O. Box 10412

Palo Alto, CA 94303

Los Alamos National Laboratory

Attn: B. E. Boyack, K-551

P. O. Box 1663

Los Alamos, NM 87545

Oak Ridge National Laboratory (11)

P. O. Box 2009

Oak Ridge, TN 37831-8057

Attn: S. R. Greene, MS-8057

R. H. Morris, MS- 8057

S. E. Fisher, MS- 8057

R. Sanders, MS-8057

T. L. Heatherly, MS- 8057

S. A. Hodge, MS- 8057

C. R. Hyman, MS- 8057

B. W. Patton, MS-8057

D. B. Simpson, MS- 8057

R. P. Taleyarkhan, MS-8057

M. L. Tobias, MS- 8088

P. O. Box 2009

Oak Ridge, TN 37831-8057 
Andrzej Drozd

Nuclear Regulatory Commission

OWFN, MS 8E1

11555 Rockville Pike

Rockville, MD 20852

W. P. Barthold

Barthold \& Associates

132 Seven Oaks Drive

Knoxville, TN 37922

K. C. Wagner

Science Applications Intl. Corp.

2109 Air Park Rd. SE

Albuquerque, NM 87106

Savannah River Laboratory (2)

Attn: B. DeWald

D. Allison

Westinghouse Savannah River Co.

Bldg. 773-41A

Aiken, SC 29808-0001

Westinghouse Hanford Co. (2)

Attn: D. Ogden

O. Wang

P. O. Box 1970

Richland, WA 99352

General Electric Company (4)

Knolls Atomic Pwer Laboratory

Attn: D. F. McMullan

G. H. Epstein

E. Menard

Joe Alfeo

Bldg. F3, Room 8

P. O. Box 1072

Schenectady, NY 12301-1072

Bettis Atomic Power Laboratory (4)

Attn: Mark Riley

Vincent Baiamonte

Joe Semancik

P. O. Box 79

West Mifflin, PA 15122 
Mohsen Khatib-Rahbar

Energy Research Inc.

P. O. Box 2034

Rockville, MD 20852

V. K. Dhir

2445 22nd Street

Santa Monica, CA 90403

R. Viskanta

Purdue University

Heat Transfer Laboratory

School of Mechanical Engineering

West Lafayette, IN 47907

Dr. Jim Gieseke

Battelle Memorial Institute

505 King Ave.

Columbus, Ohio 43201

M. A. Kenton

Gabor, Kenton \& Associates

770 Pasquinelli Drive

Suite 426

Westmont, IL 60559

University of California (2)

Attn: W. H. Amarasooriya

T. Theofanous

ERC-CRSS

Santa Barbara, CA 93106

F. E. Haskin

University of New Mexico

Department of Chemical and Nuclear Engineering

Albuquerque, NM 87131

J. C. Lee

University of Michigan

Dept. of Nuclear Engineering

Cooley Building, North Campus

College of Engineering

Ann Arbor, MI 48109-2104 
University of Wisconsin (2)

Dept. of Nuclear Engineering

Attn: M. L. Corradini

G. A. Moses

Engineering Research Building

1500 Johnson Drive

Madison, WI 53706

Ramu K. Sundaram

Manager, LOCA Analysis Group

Nuclear Engineering

Yankee Atomic Electric Company

580 Main Street

Bolton, MA 01740

CEGA

Attn: John Bolin

P. O. Box 85608

San Diego, CA 92186-9784

M. Plys

Fauske \& Associates

16W070 West 83rd Street

Burr Ridge, IL 60521

Nick Trikouros

GPU Nuclear Corporation

One Upper Pond Road

Parsippany, NJ 07054

B. Raychaudhuri

Nebraska Public Power District

PRA \& Engineering Review Group

P. O. Box 499

Columbus, NE 68601

Frank Elia

Stone \& Webster Engineering Corp.

245 Summer Street

Boston, MA 02210 
Samir S. Girgis

Atomic Energy of Canada Limited

CANDU Operations

Sheridan Park Research Community

Mississagua, Ontario

CANADA L5K1B2

Paul J. Fehrenbach

Chalk River Nuclear Laboratories

Fuel Engineering Branch, RSR Division

Chalk River, Ontario

CANADA KOJ $1 \mathrm{~J} 0$

Dr. Bohumír Kujal

Department of Reactor Technology

Nuclear Research Institute Re'z plc

25068 Rež

CZECH REPUBLIC

Andrej Mitro

Institute of Radioecology and Applied Nuclear Techniques

Garbiarska 2

P. O. Box A-41

04061 Košice

CHECHOSLOVAKIA

Shih-Kuei Cheng

Atomic Energy Council, Nuclear Safety

67, Lane 144, Keelung Rd. Sec. 4

Taipei, Taiwan

REPUBLIC OF CHINA

Lainsu Kao, Associate Scientist

Atomic Energy Council

Institute of Nuclear Energy Research

P. O. Box 303

Lung-'Tan, 32500

Taiwan

REPUBIIC OF CHINA 
Technical Research Centre of Finland (3)

Nuclear Engineering Laboratory

Attn: Lasse Mattila

Ilona Lindholm

Esko Pekkarinen

P. O. Box 208 ('Tekniikantie 4)

SF-002151 Espoo

FINLAND

Jorma V. Sandberg

Finnish Center Radiation \& Nucl. Safety,

Dept. of Nuclear Safety

P. O. Box 268

SF-00101 Helsinki

FINLANI)

Akihide Hidaka

Safety Research Department

Reactor Accident Studies and Modelling Branch

DRS/SEMAR

('adarache Nuclear Center

13108 Saint-Paul-Lez-Durance Cedex

FRANCE

Dr. Lothar Wolf

Battelle Institute EV

AM Rornerhof 35

D) -6000

Frankfurt/Main90

GERMANY

Gesellschaft fur Anlagen- und Reaktorsicherheit (3)

Attn: Ulrich Erven

Walter Erdmann

Manfred Firnhaber

Schwertnergasse 1

D-5000 Koln 1

GERMANY 
Kernforschungzentrum, Karlsruhe (3)

Attn: P. Hofmann

Werner Scholtyssek

Philipp Schmuck

P. O. Box 3640

D. 7500 Karlsruhe 1

GERMANY

Udo Brockmeier

University of Bochum

Energietechnik

IB-4-128

D-4630 Bochum

GERMANY

György Gyenes

Central Research Institute for Physics

Institute for Atomic Energy Research

H-1525 Budapest, P. (). Box 49

HUNGARY

Joint Research Center

Commission of the European Communities

Attn: Alan Jones

Iain Shepherd

Safety 'Technology Institute

21020 Ispra (Va)

ITALY

Giovanni Saponaro

ENEA

Natl. Comm. for R\&D of Nuclear Energy

Via Vitaliano Brancati, 48

00144 Rome

I'TALY

Japan Atomic Energy Research Institute (2)

Attn: Kunihisa Soda

Jun Sugimoto

Norihiro Yamano

Tokai-mura, Naka-gun, Ibaraki-ken

319-11, JAPAN 
Dr. Masayoshi Shiba, Director General

Institute of Nuclear Safety

Nuclear Power Engineering Corporation

Fujita Kankou Toranoman Bldg. 7F

3-17-1, Toranoman

Minato-Ku, Tokyo, 105

JAPAN

Masao Ogino

Mitsubishi Atomic Power Industries

4-1 Shibakoen 2-Chome

Minatoku Tokyo

JAPAN

Hidetoshi Okada

Nuclear Power Engineering Corporation

3-17-1, Toranomon Bldg. 5F

Minato-ku, Tokyo 105

JAPAN

Hirohide Oikawa

Toshiba Corporation

8, Shin-Sugita, Isogo-ku

Yokohama

JAPAN

Korea Atomic Energy Research Inst. (3)

Attn: Kun-Joong Yoo

Song-Won Cho

Dong-Ha Kim

P. O. Box 7, Daeduk Danji

Taejon

SOUTH KOREA 305-353

Jae Hong Park

Safety Assessment Department

Korea Atomic Energy Research Institute

P. O. Box 16, Daeduk-Danji

Taejon

SOUTH KOREA 305-353 
Netherlands Energy Research Foundation (2)

Attn: Karel J. Brinkmann

E. J. Velema

P. O. Box 1

1755 ZG Petten

THE NETHERLANDS

Dr. Valery F. Strizhov

Russian Academy of Science

Institute of Nuclear Safety

Moscow, G. Tulsky, 52

113191, RUSSIA

Universidad Politecnica de Madrid (2)

Attn: Augustin Alonzo Santos

Francisco Martin

E.T.S. Ingenieros Industriales

Jose Gutierrez Abascal, 2

28006 Madrid

SPAIN

Juan Bagues

Consejo de Seguridad Nuclear

Justo Dorado, 11

28040, Madrid

SPAIN

Oddbjörn Sandervåg

Statens Kärnkraftinspektion

Swedish Nuclear Power Inspectorate

Box 2710610252 Stockholm

SWEDEN

L. Hammar, Director

Division of Research

Swedish Nuclear Power Inspectorate

Statens Karnkraftinspektion

Sehlstedtsgatan 11

Box 27106

S-102-50 Stockholm

SWEDEN 
Swiss Federal Nuclear Safety Inspectorate (4)

Attn: S. Chakraborty

Sang Lung Chan

U. Schmocker

H. P. Isaak

CH-5232 Villigen-HSK

SWITZERLAND

United Kingdom Atomic Energy Agency (3)

Winfrith Technology Center

Attn: T. Haste

S. R. Kinnersley

D. W. Sweet

Winfrith, Dorchester, Dorset

UNITED KINGDOM, DTS 8DH

United Kingdom Atomic Energy Authority (2)

Safety \& Reliability Directorate

Attn: M. I. Robertson

C. Wheatley

Wigshaw Lane, Culcheth, Warrington

Cheshire, WA3 4NE

UNITED KINGDOM

Geoffrey A. Brown

AEA Technology

Consultancy Services

'Thomson House, Risley, Warrington

Cheshire, WA3 6AT

UNITED KINGDOM 
Internal Distribution:

6400 N. R. Ortiz

6403 W. A. von Riesemann

6404 D. A. Powers

6412 A. L. Camp

6412 S. E. Dingman

$6413 \mathrm{~F}$. T. Harper

6414 J. E. Kelly

6418 S. L. Thompson (10)

6418 R. K. Cole

6418 A. A. Elsbernd

6418 L. N. Kmetyk

6418 R. C. Smith

6418 D. S. Stuart

6418 R. M. Summers

6418 T. J. Tautges (10)

6422 M. D Allen

$6423 \mathrm{~K}$. O. Reil

6423 R. O. Gauntt

6423 R. C. Schmidt

6429 K. E. Washington

7141 Technical Library (5)

7151 Technical Publications

7613-2 Document Processing for DOE/OSTI (10)

8523-2 Central Technical Files 

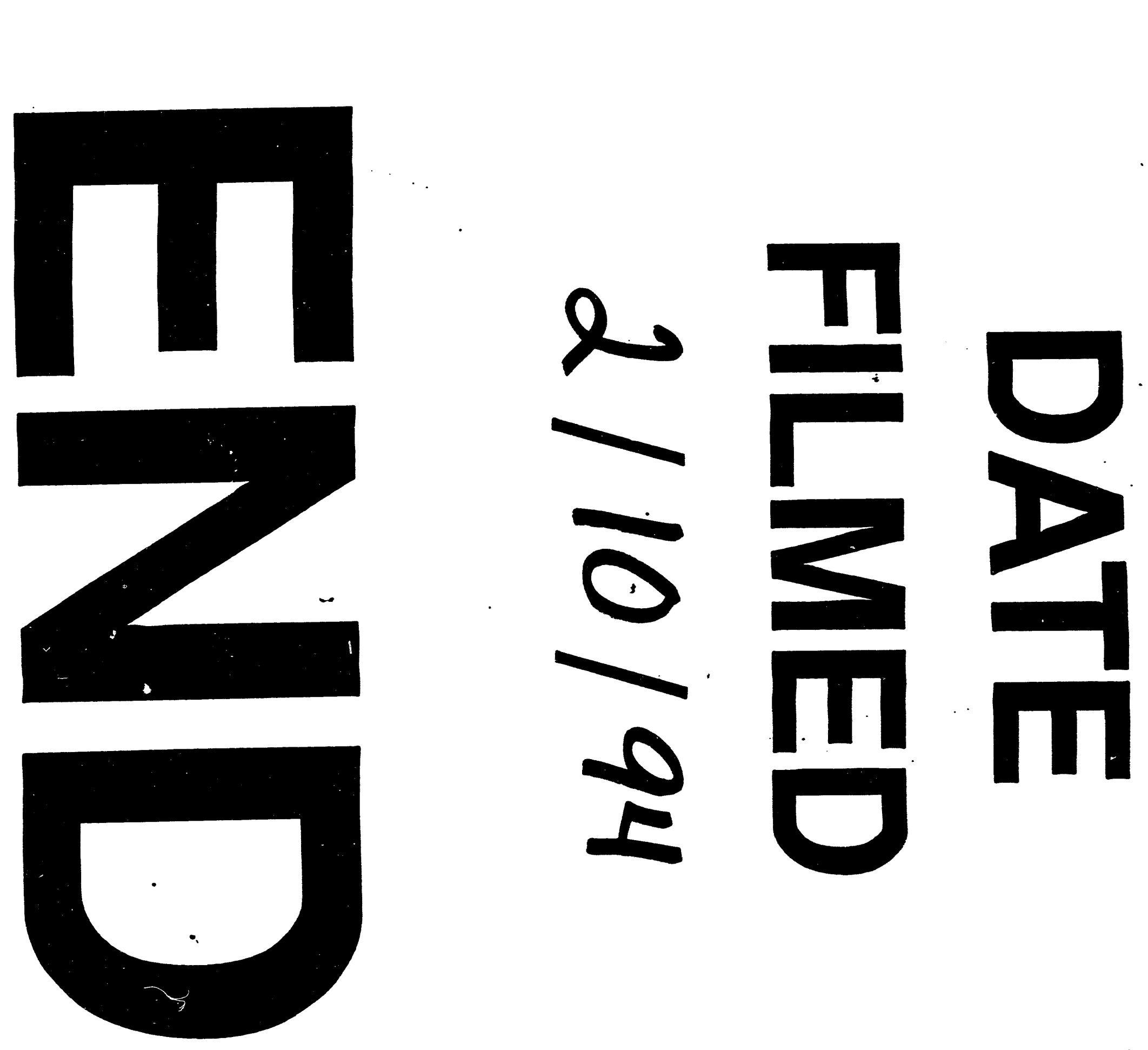